\title{
Synthesis of Pyridylsulfonium Salts and their Application in the Formation of Functionalized Bipyridines
}

\author{
Vincent K. Duong, Alexandra M. Horan, Eoghan M. McGarrigle*
}

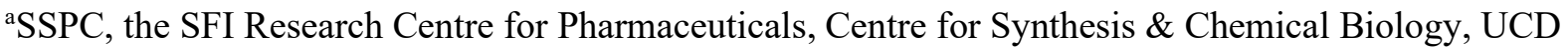
School of Chemistry, University College Dublin, Belfield, Dublin 4, Ireland.

\section{Supporting information}

\section{Table of Contents}

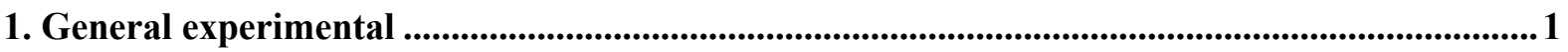

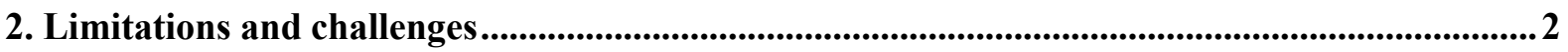

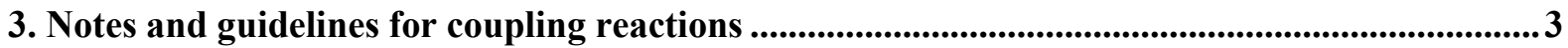

4. Synthesis of sulfides $1 \mathrm{a}-\mathrm{i}$ and halopyridine starting material $\mathrm{S1}$.................................................... 4

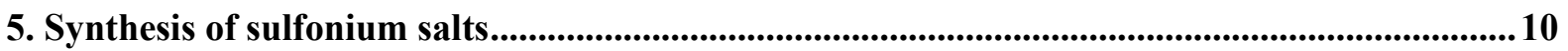

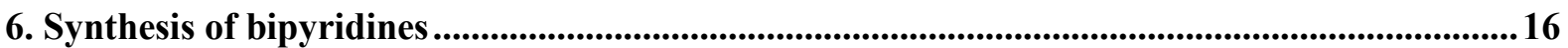

7. NMR Spectra ........................................................................................................................................................37

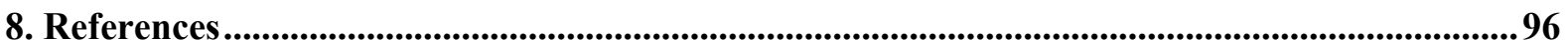




\section{General experimental}

Chemicals were purchased and used without further purification unless otherwise stated. 2Methyltetrahydrofuran was transferred to a Schlenk tube, dried over freshly activated $4 \AA$ molecular sieves and stored under $\mathrm{N}_{2}$. Solvents were dried using a Grubbs-type still, a Pure Solv-400-3-MD solvent purification system supplied by Innovative Technology Inc. design and stored in Strauss flasks over activated $4 \AA$ molecular sieves. Diphenyliodonium trifluoromethanesulfonate, ${ }^{1}$ 2-bromo-5-(1,3dioxolan-2-yl)pyridine ${ }^{2}$ and 2-bromo-5-((trimethylsilyl)ethynyl)pyridine ${ }^{3}$ were prepared according to literature procedures.

Reactions requiring anhydrous conditions were performed under $\mathrm{N}_{2}$; glassware was flame-dried immediately prior to use and allowed to cool under reduced pressure. Reaction monitoring by TLC was performed on Merck pre-coated Kieselgel $60 \mathrm{~F}_{254}$ aluminium plates. Visualization was accomplished under UV light (254 nm). Flash column chromatography (FCC) was performed using either silica gel [Davisil, 230-400 mesh $(40-63 \mu \mathrm{m})$ ] or using a Biotage Isolera ${ }^{\mathrm{TM}}$ UV-VIS Flash Purification System Version 2.3.1 with SNAP Ultra $(25 \mu \mathrm{m})$, SNAP KP-Sil $(50 \mu \mathrm{m})$ or SNAP KP-NH $(50 \mu \mathrm{m})$ prepacked silica cartridges. High-resolution mass spectra were run on a Waters Micromass GCT system or on an Agilent 6546 QTOF system in electrospray ionization mode (ESI). Extracts were concentrated in vacuo using both a rotary evaporator (bath temperatures up to $55^{\circ} \mathrm{C}$ ), and a high vacuum line at room temperature. ${ }^{1} \mathrm{H}$ NMR, ${ }^{13} \mathrm{C}$ NMR and ${ }^{19} \mathrm{~F}$ NMR spectra were measured in the solvent stated at $300 \mathrm{MHz}$, $400 \mathrm{MHz}$ or $500 \mathrm{MHz}$. Chemical shifts $(\delta)$ are quoted in parts per million (ppm) referenced to residual solvent peak (e.g., $\mathrm{CDCl}_{3}:{ }^{1} \mathrm{H}-7.26 \mathrm{ppm}$ and ${ }^{13} \mathrm{C}-77.16 \mathrm{ppm}$ ) or TMS $\left({ }^{1} \mathrm{H}-0.00 \mathrm{ppm}\right)$ and coupling constants $(J)$ are given in Hertz. Multiplicities are abbreviated as: b (broad), s (singlet), d (doublet), t (triplet), q (quartet), m (multiplet) or combinations thereof. Assignments were made, where necessary, with the aid of COSY, HSQC, HMBC and NOESY NMR experiments. 


\section{Limitations and challenges}

Table 1: Limitations and challenging substrates with the methodology

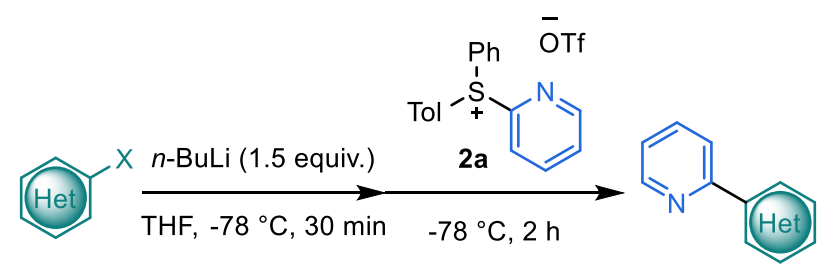<smiles>FC(F)(F)c1ccnc(-c2ccccn2)c1</smiles>

Formation of 4 fluorinated bipyridines<smiles>c1ccc(-c2ccnc3ccccc23)nc1</smiles>

Formation of 2,4bipyridines<smiles>c1ccc(-c2ncccn2)nc1</smiles>

Unsuccesful pyrimidine coupling

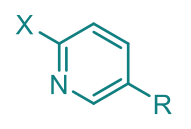

$\mathrm{R}=\mathrm{CN}$ or $\mathrm{NO}_{2}$

Not compatible with organolithium chemistry<smiles>FC(F)(F)c1ccc(-c2ccccn2)nc1</smiles>

Low yielding<smiles>Pc1cccc(-c2ccccn2)n1</smiles>

Low yielding<smiles>Brc1cncc(-c2ccccn2)c1</smiles>

$20 \%$ yield

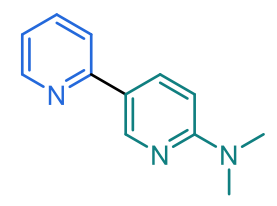

No desired product<smiles>Brc1cccc(-c2cccc(Br)n2)n1</smiles>

Low yielding<smiles>Clc1ccnc(-c2cccc(Br)n2)c1</smiles>

No desired product<smiles>Brc1cccc(-c2nccc3ccccc23)n1</smiles>

Unreactive<smiles>[Se]c1ccc(-c2cccc(Br)n2)cn1</smiles>

No reaction<smiles>FC(F)(F)c1ccc(-c2ccc(C(F)(F)F)cn2)nc1</smiles>

Mixture of products, difficult to purify<smiles>COc1ccc(-c2ccc(C(F)(F)F)cn2)nc1</smiles>

Low yielding<smiles>COc1ccc(-c2ccc(C(F)(F)F)cn2)nc1</smiles>

No reaction<smiles>CN(C)c1ccc(-c2ccc(N(C)C)cn2)nc1</smiles>

Mixture of products, difficult to purify 


\section{Notes and guidelines for coupling reactions}

1. The ligand coupling sequence produces at least three products: sulfide, dehalogenated material and bipyridine, making the purification potentially tricky. However, the desired bipyridine product is generally the most polar material by TLC relative to sulfide and dehalogenated pyridine starting material.

2. Some sulfonium salts can require some stirring before they fully dissolve in THF. We recommend preparing a solution of the pyridylsulfonium salt by stirring in THF in a separate flask while the organolithium is generated in the reaction flask.

3. Amine-containing bipyridines can be rather basic and require the addition of $\mathrm{Et}_{3} \mathrm{~N}(1 \%)$ in the eluent for flash column chromatography.

4. 2,2-Bipyridines are generally less polar than 2,3-bipyridines on silica.

5. For $\mathrm{S}_{\mathrm{N}} \mathrm{Ar}$-active substrates (i.e. $\mathrm{Cl}-, \mathrm{Br}$ - or F-containing pyridines), addition of sulfonium to the reaction flask can be carried out after $15 \mathrm{~min}$ rather than $30 \mathrm{~min}$. This minimizes the amount of homocoupling (reaction between lithiated pyridine with halogenated pyridine starting material).

6. Iodopyridines can be lithiated in the presence of sulfonium salt, removing the need for a sulfonium addition step. 


\section{Synthesis of sulfides 1a-i and halopyridine starting material S1}

General procedure A: 4-methylbenzenethiol (1 equiv.) was added to a round bottomed flask, followed by halopyridine (1 equiv.) and $\mathrm{H}_{2} \mathrm{O}(0.5 \mathrm{M})$. The reaction was heated under reflux for $24 \mathrm{~h}$ (the flask sitting in an aluminium block placed on a stirrer hotplate). Then, the reaction was allowed to cool, followed by extraction with EtOAc. The combined organic layers were washed with water and brine and dried over $\mathrm{Na}_{2} \mathrm{SO}_{4}$. The solvent was evaporated and the product was isolated by FCC.

General procedure B: 4-methylbenzenethiol (1 equiv.) was added to a crimptop vial followed by halopyridine (1 equiv.) and $\mathrm{K}_{2} \mathrm{CO}_{3}$ (1.1 equiv.) and closed. The vial was evacuated and purged with $\mathrm{N}_{2}$ three times. Anhydrous DMF (0.8-1 M wrt thiol/halopyridine) was added. The reaction was heated to $100{ }^{\circ} \mathrm{C}$ for $24 \mathrm{~h}$ (the flask sitting in an aluminium block placed on a stirrer hotplate). Then the reaction was allowed to cool, followed by the addition of water and extraction with EtOAc. The combined organic layers were washed with water and brine and dried over $\mathrm{Na}_{2} \mathrm{SO}_{4}$. Details of purification are given below for each compound.

\section{2-(p-Tolylthio)pyridine 1a}<smiles>Cc1ccc(Sc2ccccn2)cc1</smiles>

Prepared from General procedure A with 4-methylbenzenethiol (14.0 g, $113 \mathrm{mmol})$, 2-bromopyridine $(10.8 \mathrm{~mL}, 113 \mathrm{mmol})$ and $\mathrm{H}_{2} \mathrm{O}(226 \mathrm{~mL})$. The reaction was extracted with EtOAc $(3 \times 200 \mathrm{~mL})$. The combined organic layers were washed with water $(200 \mathrm{~mL})$ and brine $(200 \mathrm{~mL})$ and dried over $\mathrm{Na}_{2} \mathrm{SO}_{4}$. The solvent was evaporated and purification by $\mathrm{FCC}\left(10 \% \mathrm{Et}_{2} \mathrm{O}\right.$ in pentane) gave pyridylsulfide $\mathbf{1 a}$ as a yellow oil (21.05 g, 93\%).

${ }^{1} \mathrm{H}$ NMR (400 MHz, Chloroform- $d$ ): $\delta 8.41$ (ddd, $\left.J=4.9,2.0,0.9 \mathrm{~Hz}, 1 \mathrm{H}\right), 7.49(\mathrm{~d}, J=8.1 \mathrm{~Hz}, 2 \mathrm{H})$, 7.42 (ddd, $J=8.2,7.4,1.9 \mathrm{~Hz}, 1 \mathrm{H}), 7.24$ (d, $J=7.9 \mathrm{~Hz}, 2 \mathrm{H}), 6.97$ (ddd, $J=7.5,4.9,1.1 \mathrm{~Hz}, 1 \mathrm{H}), 6.84$ $(\mathrm{dt}, J=8.1,1.0 \mathrm{~Hz}, 1 \mathrm{H}), 2.40(\mathrm{~s}, 3 \mathrm{H})$.

${ }^{13} \mathrm{C}$ NMR (101 MHz, Chloroform-d) $\delta$ 162.4, 149.6, 139.6, 136.7, 135.4, 130.6, 127.4, 121.0, 119.7, 21.5.

Spectra were consistent with literature data. ${ }^{4}$

\section{3-Methyl-2-(p-tolylthio)pyridine 1b}<smiles>Cc1ccc(Sc2ncccc2C)cc1</smiles>

Prepared from General procedure A with 4-methylbenzenethiol (1.00 g, $8.05 \mathrm{mmol})$, 3-bromo-2methylpyridine $(0.89 \mathrm{~mL}, 8.0 \mathrm{mmol})$ and $\mathrm{H}_{2} \mathrm{O}(15 \mathrm{~mL})$. The reaction was extracted with EtOAc $(3 \times 20$ 
$\mathrm{mL})$. The combined organic layers were washed with water $(20 \mathrm{~mL})$ and brine $(20 \mathrm{~mL})$ and dried over $\mathrm{Na}_{2} \mathrm{SO}_{4}$. The solvent was evaporated and purification by $\mathrm{FCC}\left(5 \% \mathrm{Et}_{2} \mathrm{O}\right.$ in pentane) gave pyridylsulfide $\mathbf{1 b}$ as a clear oil $(0.93 \mathrm{~g}, 54 \%)$.

TLC: $R_{\mathrm{f}}=0.33\left(10 \% \mathrm{Et}_{2} \mathrm{O}\right.$ in pentane $)$.

${ }^{1} \mathrm{H}$ NMR (500 MHz, Chloroform- $d$ ) $\delta 8.22(\mathrm{ddd}, J=4.9,1.9,0.8 \mathrm{~Hz}, 1 \mathrm{H}), 7.41(\mathrm{~d}, J=8.1 \mathrm{~Hz}, 2 \mathrm{H}), 7.37$ (ddd, $J=7.4,1.7,0.8 \mathrm{~Hz}, 1 \mathrm{H}), 7.23-7.17(\mathrm{~m}, 2 \mathrm{H}), 6.95(\mathrm{dd}, J=7.5,4.8 \mathrm{~Hz}, 1 \mathrm{H}), 2.38\left(\mathrm{~s}, 3 \mathrm{H}, \mathrm{CH}_{3}\right)$, $2.36\left(\mathrm{~s}, 3 \mathrm{H}, \mathrm{CH}_{3}\right)$.

${ }^{13} \mathrm{C}$ NMR (126 MHz, Chloroform-d) $\delta 158.3(\mathrm{C}), 147.4(\mathrm{CH}), 138.5(\mathrm{C}), 137.1(\mathrm{CH}), 134.5(\mathrm{CH}), 131.6$ (C), $130.0(\mathrm{CH}), 127.7(\mathrm{C}), 120.3(\mathrm{CH}), 21.5\left(\mathrm{CH}_{3}\right), 19.2\left(\mathrm{CH}_{3}\right)$.

HRMS (ESI-TOF) $m / z$ : [M+H] ${ }^{+}$Calcd for $\mathrm{C}_{13} \mathrm{H}_{14} \mathrm{NS} 216.0841$; Found 216.0852 .

\section{1-(p-Tolylthio)isoquinoline 1c}<smiles>Cc1ccc(Sc2nccc3ccccc23)cc1</smiles>

Prepared from General procedure B with 4-methylbenzenethiol (1.52 g, $12.2 \mathrm{mmol})$, 1chloroisoquinoline (2.0 g, $12 \mathrm{mmol}), \mathrm{K}_{2} \mathrm{CO}_{3}(1.86 \mathrm{~g}, 13.4 \mathrm{mmol})$ and DMF (12 mL). The reaction was extracted with EtOAc $(3 \times 25 \mathrm{~mL})$. The combined organic layers were washed with water $(50 \mathrm{~mL})$ and brine $(50 \mathrm{~mL})$ and dried over $\mathrm{Na}_{2} \mathrm{SO}_{4}$. The solvent was evaporated and purification by FCC $\left(10 \% \mathrm{Et}_{2} \mathrm{O}\right.$ in pentane) gave pyridylsulfide $1 \mathrm{c}$ as a yellow oil (3.02 $\mathrm{g}, 98 \%)$.

${ }^{1} \mathrm{H}$ NMR (500 MHz, Chloroform- $d$ ) $\delta 8.36$ (dd, $\left.J=8.4,1.0 \mathrm{~Hz}, 1 \mathrm{H}\right), 8.23(\mathrm{~d}, J=5.7 \mathrm{~Hz}, 1 \mathrm{H}), 7.77$ (dt, $J=8.2,0.9 \mathrm{~Hz}, 1 \mathrm{H}), 7.68(\mathrm{ddd}, J=8.1,6.9,1.2 \mathrm{~Hz}, 1 \mathrm{H}), 7.60(\mathrm{ddd}, J=8.3,6.9,1.3 \mathrm{~Hz}, 1 \mathrm{H}), 7.53-$ 7.47 (m, 2H), 7.36 (dd, $J=5.7,0.9 \mathrm{~Hz}, 1 \mathrm{H}), 7.27-7.21(\mathrm{~m}, 2 \mathrm{H}), 2.39\left(\mathrm{~s}, 3 \mathrm{H}, \mathrm{CH}_{3}\right)$.

${ }^{13} \mathrm{C}$ NMR (126 MHz, Chloroform- $d$ ) $\delta 160.0,142.6,138.9,136.0,135.2,130.5,130.18,127.4,127.3$, 127.1, 126.9, 125.1, 118.3, $21.5\left(\mathrm{CH}_{3}\right)$.

Spectra are consistent with literature data. ${ }^{5}$ 


\section{2-(p-Tolylthio)-4-(trifluoromethyl)pyridine 1d}<smiles>Cc1ccc(Sc2cc(C(F)(F)F)ccn2)cc1</smiles>

Prepared from General procedure B with 4-methylbenzenethiol (1.10 g, $8.85 \mathrm{mmol})$, 2-bromo-4(trifluoromethyl)pyridine $(1.10 \mathrm{~mL}, 8.85 \mathrm{mnol}), \mathrm{K}_{2} \mathrm{CO}_{3}(1.34 \mathrm{~g}, 9.73 \mathrm{mmol})$ and $\mathrm{DMF}(3 \mathrm{~mL})$. The reaction was extracted with EtOAc $(3 \times 10 \mathrm{~mL})$. The combined organic layers were washed with water $(20 \mathrm{~mL})$ and brine $(20 \mathrm{~mL})$ and dried over $\mathrm{Na}_{2} \mathrm{SO}_{4}$. The solvent was evaporated and purification by FCC (10\% $\mathrm{Et}_{2} \mathrm{O}$ in pentane) gave pyridylsulfide $1 \mathrm{~d}$ as a yellow oil $(2.30 \mathrm{~g}, 97 \%)$.

TLC: $R_{\mathrm{f}}=0.45\left(10 \% \mathrm{Et}_{2} \mathrm{O}\right.$ in pentane $)$.

${ }^{1} \mathrm{H}$ NMR (500 MHz, Chloroform- $d$ ) $\delta 8.56(\mathrm{dt}, J=5.1,0.7 \mathrm{~Hz}, 1 \mathrm{H}), 7.49(\mathrm{~d}, J=8.1 \mathrm{~Hz}, 2 \mathrm{H}), 7.31-$ $7.27(\mathrm{~m}, 2 \mathrm{H}), 7.16(\mathrm{ddd}, J=5.1,1.5,0.7 \mathrm{~Hz}, 1 \mathrm{H}), 7.03(\mathrm{dt}, J=1.6,0.8 \mathrm{~Hz}, 1 \mathrm{H}), 2.42\left(\mathrm{~s}, 3 \mathrm{H}, \mathrm{CH}_{3}\right)$.

${ }^{13} \mathrm{C}$ NMR (126 MHz, Chloroform- $d$ ) $\delta 164.5(\mathrm{C}), 150.6(\mathrm{CH}), 140.4(\mathrm{C}), 139.1$ (q, $J=34.0 \mathrm{~Hz}, C \mathrm{CF}_{3}$ ), $135.5(\mathrm{CH}), 130.9(\mathrm{CH}), 125.8(\mathrm{C}), 122.7$ (q, $\left.J=273.3 \mathrm{~Hz}, \mathrm{CF}_{3}\right), 116.2$ (q, $\left.J=3.8 \mathrm{~Hz}, \mathrm{CH}\right), 115.1$ (q, $J=3.5 \mathrm{~Hz}, \mathrm{CH}), 21.5\left(\mathrm{CH}_{3}\right)$.

${ }^{19} \mathrm{~F}$ NMR (470 MHz, Chloroform- $d$ ) $\delta-65.11$.

HRMS (ESI-TOF) $m / z:[\mathrm{M}+\mathrm{H}]^{+}$Calcd for $\mathrm{C}_{13} \mathrm{H}_{11} \mathrm{~F}_{3} \mathrm{NS} 270.0559$; Found 270.0560 .

\section{5-Nitro-2-(p-tolylthio)pyridine 1e}<smiles>Cc1ccc(Sc2ccc([N+](=O)[O-])cn2)cc1</smiles>

Prepared from General procedure A with 4-methylbenzenethiol (1.0 g, $8.05 \mathrm{mmol})$, 2-bromo-5nitropyridine $(1.63 \mathrm{~g}, 8.05 \mathrm{mmol})$ and $\mathrm{H}_{2} \mathrm{O}(15 \mathrm{~mL})$. The reaction was extracted with EtOAc $(3 \mathrm{x} 20$ $\mathrm{mL})$. The combined organic layers were washed with water $(20 \mathrm{~mL})$ and brine $(20 \mathrm{~mL})$ and dried over $\mathrm{Na}_{2} \mathrm{SO}_{4}$. The solvent was evaporated to give pyridylsulfide 1e as a colourless oil without further purification (1.98 g, quantitative yield).

${ }^{1} \mathrm{H}$ NMR (500 MHz, Chloroform- $d$ ) $\delta 9.21(\mathrm{dd}, J=2.7,0.7 \mathrm{~Hz}, 1 \mathrm{H}), 8.17(\mathrm{dd}, J=8.9,2.7 \mathrm{~Hz}, 1 \mathrm{H}), 7.53$ -7.47 (m, 2H), $7.36-7.29$ (m, 2H), 6.91 (dd, $J=9.0,0.7 \mathrm{~Hz}, 1 \mathrm{H}), 2.44\left(\mathrm{~s}, 3 \mathrm{H}, \mathrm{CH}_{3}\right)$.

${ }^{13} \mathrm{C}$ NMR (126 MHz, Chloroform-d) $\delta 171.1,145.2,141.14,141.08,135.7,131.3,131.2,125.0,119.8$, $21.6\left(\mathrm{CH}_{3}\right)$.

HRMS (ESI-TOF) $m / z$ : [M+H] $]^{+}$Calcd for $\mathrm{C}_{12} \mathrm{H}_{11} \mathrm{~N}_{2} \mathrm{O}_{2} \mathrm{~S}$ 247.0536; Found 247.0538. 
Spectra are consistent with literature data. ${ }^{6}$

\section{2-(p-Tolylthio)-5-(trifluoromethyl)pyridine $1 \mathrm{f}$}<smiles>Cc1ccc(Sc2ccc(C(F)(F)F)cn2)cc1</smiles>

Prepared from General procedure B with 4-methylbenzenethiol (1.0 g, $8.0 \mathrm{mmol})$, 2-bromo-5(trifluoromethyl)pyridine $(1.32 \mathrm{~g}, 8.0 \mathrm{mmol}), \mathrm{K}_{2} \mathrm{CO}_{3}(1.22 \mathrm{~g}, 8.8 \mathrm{mmol})$ and DMF $(10 \mathrm{~mL})$. The reaction was extracted with EtOAc $(3 \times 20 \mathrm{~mL})$. The combined organic layers were washed with water $(30 \mathrm{~mL})$ and brine $(30 \mathrm{~mL})$ and dried over $\mathrm{Na}_{2} \mathrm{SO}_{4}$. The solvent was evaporated to give pyridylsulfide 1f as a white solid which was used without further purification $(2.12 \mathrm{~g}, 98 \%)$.

${ }^{1} \mathrm{H}$ NMR (500 MHz, Chloroform- $d$ ) $\delta 8.64(\mathrm{dt}, J=2.6,0.9 \mathrm{~Hz}, 1 \mathrm{H}), 7.66-7.55(\mathrm{~m}, 1 \mathrm{H}), 7.50(\mathrm{~d}, J=$ $8.1 \mathrm{~Hz}, 2 \mathrm{H}), 7.35-7.27(\mathrm{~m}, 2 \mathrm{H}), 6.89(\mathrm{dt}, J=8.5,0.8 \mathrm{~Hz}, 1 \mathrm{H}), 2.42\left(\mathrm{~s}, 3 \mathrm{H}, \mathrm{CH}_{3}\right)$.

${ }^{13} \mathrm{C}$ NMR (126 MHz, Chloroform- $d$ ) $\delta 167.6(\mathrm{C}), 146.5$ (q, $\left.J=4.3 \mathrm{~Hz}, \mathrm{CH}\right), 140.50(\mathrm{C}), 135.7$ (CH), 133.5 (q, $J=3.5 \mathrm{~Hz}, \mathrm{CH}), 130.9(\mathrm{CH}), 125.8(\mathrm{C}), 123.8$ (q, $\left.J=271.8 \mathrm{~Hz}, \mathrm{CF}_{3}\right), 122.5$ (q, $J=33.4 \mathrm{~Hz}$, $\left.\mathrm{CCF}_{3}\right), 120.0(\mathrm{CH}), 21.5\left(\mathrm{CH}_{3}\right)$.

${ }^{19}$ F NMR (470 MHz, Chloroform- $d$ ) $\delta-62.21$.

HRMS (ESI-TOF) $m / z$ : [M+H] $]^{+}$Calcd for $\mathrm{C}_{13} \mathrm{H}_{11} \mathrm{~F}_{3} \mathrm{NS} 270.0559$; Found 270.0558 .

\section{5-Methoxy-2-(p-tolylthio)pyridine 1g}<smiles>COc1ccc(Sc2ccc(C)cc2)nc1</smiles>

Prepared from General procedure A with slight modifications, with 4-methylbenzenethiol $(1.19 \mathrm{~g}, 9.60$ mmol), 2-bromo-5-methoxypyridine $(1.50 \mathrm{~g}, 7.98 \mathrm{mmol})$ and $\mathrm{H}_{2} \mathrm{O}(8 \mathrm{~mL})$. The reaction was heated under reflux for $48 \mathrm{~h}$ (the flask sitting in an aluminium block placed on a stirrer hotplate). The reaction was extracted with EtOAc $(3 \times 30 \mathrm{~mL})$. The combined organic layers were washed with water $(30 \mathrm{~mL})$ and brine $(30 \mathrm{~mL})$ and dried over $\mathrm{Na}_{2} \mathrm{SO}_{4}$. The solvent was evaporated and purification by FCC $(10 \%$ $\mathrm{Et}_{2} \mathrm{O}$ in pentane) gave pyridylsulfide $\mathbf{1 g}$ a white solid (0.95 g, 51\%).

TLC: $R_{\mathrm{f}}=0.10\left(10 \% \mathrm{Et}_{2} \mathrm{O}\right.$ in pentane $)$.

${ }^{1} \mathrm{H}$ NMR (500 MHz, Chloroform- $d$ ) $\delta 8.17(\mathrm{~d}, J=3.0 \mathrm{~Hz}, 1 \mathrm{H}), 7.41(\mathrm{~d}, J=8.2 \mathrm{~Hz}, 2 \mathrm{H}), 7.17(\mathrm{~d}, J=8.2$ $\mathrm{Hz}, 2 \mathrm{H}), 7.04$ (dd, $J=8.7,3.0 \mathrm{~Hz}, 1 \mathrm{H}), 6.94$ (d, $J=8.6 \mathrm{~Hz}, 1 \mathrm{H}), 3.80$ (s, 3H, OMe), 2.36 (s, 3H, Me).

${ }^{13} \mathrm{C}$ NMR (126 MHz, Chloroform- $d$ ) $\delta 153.8$ (COMe), $151.6(\mathrm{C}), 138.7(\mathrm{C}), 136.9(\mathrm{CH}), 134.0(\mathrm{CH})$, $130.3(\mathrm{CH}), 129.4(\mathrm{C}), 123.4(\mathrm{CH}), 122.6(\mathrm{CH}), 55.8(\mathrm{OMe}), 21.3\left(\mathrm{CH}_{3}\right)$. 
HRMS (ESI-TOF) $m / z$ : [M+H] $]^{+}$Calcd for $\mathrm{C}_{13} \mathrm{H}_{14} \mathrm{NOS} 232.0791$; Found 232.0807.

\section{$N, N$-Dimethyl-6-(p-tolylthio)pyridin-3-amine $1 \mathrm{~h}$}<smiles>CNc1ccc(Sc2ccc(C)cc2)nc1</smiles>

Prepared from General procedure B with 4-methylbenzenethiol (500 mg, $4.0 \mathrm{mmol}$ ), 6-bromo- $\mathrm{N}, \mathrm{N}$ dimethylpyridin-3-amine (804 mg, $4.0 \mathrm{mmol}), \mathrm{K}_{2} \mathrm{CO}_{3}(611 \mathrm{mg}, 4.4 \mathrm{mmol})$ and DMF (5 mL). The reaction was extracted with EtOAc $(3 \times 10 \mathrm{~mL})$. The combined organic layers were washed with water $(20 \mathrm{~mL})$ and brine $(20 \mathrm{~mL})$ and dried over $\mathrm{Na}_{2} \mathrm{SO}_{4}$. The solvent was evaporated to give pyridylsulfide 1h as a white solid which was used without further purification (814 $\mathrm{mg}, 83 \%)$.

${ }^{1} \mathrm{H}$ NMR (500 MHz, Chloroform- $d$ ) $\delta 8.04(\mathrm{dd}, J=3.2,0.6 \mathrm{~Hz}, 1 \mathrm{H}), 7.35-7.29$ (m, 2H), $7.15-7.09$ (m, 2H), $7.04(\mathrm{dd}, J=8.8,0.7 \mathrm{~Hz}, 1 \mathrm{H}), 6.87(\mathrm{dd}, J=8.7,3.2 \mathrm{~Hz}, 1 \mathrm{H}), 2.94\left(\mathrm{~s}, 6 \mathrm{H}, \mathrm{NMe}_{2}\right), 2.32(\mathrm{~s}, 3 \mathrm{H}$, $\left.\mathrm{CH}_{3}\right)$.

${ }^{13} \mathrm{C}$ NMR (126 MHz, Chloroform- $d$ ) $\delta 144.8(\mathrm{C}), 144.7(\mathrm{C}), 137.5(\mathrm{C}), 135.2(\mathrm{CH}), 132.3(\mathrm{CH}), 131.6$ $(\mathrm{C}), 130.0(\mathrm{CH}), 125.4(\mathrm{CH}), 120.3(\mathrm{CH}), 40.3\left(\mathrm{NMe}_{2}\right), 21.2\left(\mathrm{CH}_{3}\right)$.

HRMS (ESI-TOF) $m / z:[\mathrm{M}+\mathrm{H}]^{+}$Calcd for $\mathrm{C}_{14} \mathrm{H}_{17} \mathrm{~N}_{2} \mathrm{~S} 245.1107$; Found 245.1095.

\section{2-Bromo-6-(p-tolylthio)pyridine 1i}<smiles>Cc1ccc(Sc2cccc(Br)n2)cc1</smiles>

Prepared from General procedure B with slight modifications, with 4-methylbenzenethiol (1.49 g, 12.0 mmol), 2,6-dibromopyridine (1.90 g, $8.02 \mathrm{mmol}), \mathrm{K}_{2} \mathrm{CO}_{3}(2.5 \mathrm{~g}, 18 \mathrm{mmol})$ and DMF $(10 \mathrm{~mL})$. The reaction was heated to $140{ }^{\circ} \mathrm{C}$ for $3 \mathrm{~d}$ (the flask sitting in an aluminium block placed on a stirrer hotplate). The reaction was extracted with EtOAc $(3 \times 20 \mathrm{~mL})$. The combined organic layers were washed with water $(3 \times 30 \mathrm{~mL})$ and brine $(3 \times 30 \mathrm{~mL})$ and dried over $\mathrm{Na}_{2} \mathrm{SO}_{4}$. The solvent was evaporated and purification by $\mathrm{FCC}\left(5 \% \mathrm{Et}_{2} \mathrm{O}\right.$ in pentane) gave pyridylsulfide $\mathbf{1} \mathbf{i}$ as a white solid $(0.86$ g, 38\%).

TLC: $R_{\mathrm{f}}=0.42\left(5 \% \mathrm{Et}_{2} \mathrm{O}\right.$ in pentane $)$.

${ }^{1} \mathrm{H}$ NMR (500 MHz, Chloroform- $d$ ) $\delta 7.48(\mathrm{~d}, J=8.2 \mathrm{~Hz}, 2 \mathrm{H}), 7.27-7.21(\mathrm{~m}, 3 \mathrm{H}), 7.13(\mathrm{~d}, J=7.8 \mathrm{~Hz}$, $1 \mathrm{H}), 6.67(\mathrm{~d}, J=8.2 \mathrm{~Hz}, 1 \mathrm{H}), 2.40\left(\mathrm{~s}, 3 \mathrm{H}, \mathrm{CH}_{3}\right)$.

${ }^{13} \mathrm{C}$ NMR (126 MHz, Chloroform- $d$ ) $\delta 164.2(\mathrm{C}), 141.4(\mathrm{C}), 140.2(\mathrm{C}), 138.6(\mathrm{CH}), 135.6(\mathrm{CH}), 130.8$ $(\mathrm{CH}), 126.4(\mathrm{C}), 123.5(\mathrm{CH}), 119.2(\mathrm{CH}), 21.5\left(\mathrm{CH}_{3}\right)$. 
HRMS (ESI-TOF) $m / z$ : [M+Na] ${ }^{+}$Calcd for $\mathrm{C}_{12} \mathrm{H}_{10} \mathrm{BrNNaS}$ 301.9610, 303.9589; Found 301.9609, 303.9614 .

\section{(E)- and (Z)-2-Bromo-5-styrylpyridine S1}

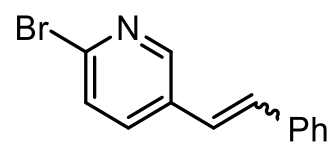

6-Bromonicotinaldehyde (200 mg, $1.08 \mathrm{mmol}$ ) and benzyltriphenylphosphonium bromide (466 mg, $1.08 \mathrm{mmol}$ ) were added to a crimptop vial and sealed. The vial was evacuated and purged with $\mathrm{N}_{2}$ three times. The reagents were dissolved in dry THF $(4 \mathrm{~mL})$ and cooled to $0{ }^{\circ} \mathrm{C}$. ${ }^{\mathrm{t}} \mathrm{BuOK}(1.08 \mathrm{~mL}, 1.0 \mathrm{M}$ in THF) was added dropwise to the vial over $2 \mathrm{~min}$. The reaction was stirred at $0{ }^{\circ} \mathrm{C}$ for $1 \mathrm{~h}$. Sat. aq. $\mathrm{NaHCO}_{3}(5 \mathrm{~mL})$ was added to quench the reaction. The product was extracted with $\mathrm{CH}_{2} \mathrm{Cl}_{2}(3 \times 20 \mathrm{~mL})$. The combined organic layers were washed with $\mathrm{H}_{2} \mathrm{O}(30 \mathrm{~mL})$ and brine $(30 \mathrm{~mL})$. The solvent was evaporated and purification by FCC $\left(5 \% \mathrm{Et}_{2} \mathrm{O}\right.$ in pentane) gave the $Z$ isomer of the bromopyridine as an opaque oil (129 mg, 46\%) and the $E$ isomer of the bromopyridine as a white solid (102 mg, 36\%).

$Z$ isomer:

TLC: $R_{\mathrm{f}}=0.55\left(25 \% \mathrm{Et}_{2} \mathrm{O}\right.$ in pentane $)$.

${ }^{1} \mathrm{H}$ NMR (400 MHz, Chloroform- $d$ ) $\delta 8.23-8.18(\mathrm{~m}, 1 \mathrm{H}), 7.35$ (ddd, $\left.J=8.3,2.5,0.6 \mathrm{~Hz}, 1 \mathrm{H}\right), 7.32-$ $7.22(\mathrm{~m}, 4 \mathrm{H}), 7.20-7.17(\mathrm{~m}, 2 \mathrm{H}), 6.79(\mathrm{~d}, J=12.2 \mathrm{~Hz}, 1 \mathrm{H}, \mathrm{CH}=\mathrm{CH}), 6.45(\mathrm{~d}, J=12.1 \mathrm{~Hz}, 1 \mathrm{H}$, $\mathrm{CH}=\mathrm{CH})$.

${ }^{13} \mathrm{C}$ NMR (101 MHz, Chloroform- $d$ ) $\left.\delta 150.5(\mathrm{CH}), 140.3(\mathrm{C}), 138.4(\mathrm{CH}), 136.2(\mathrm{C}), 133.6 \mathrm{CH}\right)$, $132.3(\mathrm{C}), 128.8(\mathrm{CH}), 128.7(\mathrm{CH}), 128.0(\mathrm{CH}), 127.6(\mathrm{CH}), 125.1(\mathrm{CH})$.

$E$ isomer:

TLC: $R_{\mathrm{f}}=0.35\left(25 \% \mathrm{Et}_{2} \mathrm{O}\right.$ in pentane).

${ }^{1} \mathrm{H}$ NMR (400 MHz, Chloroform- $d$ ) $\delta 8.44$ (d, $\left.J=2.5 \mathrm{~Hz}, 1 \mathrm{H}\right), 7.68$ (dd, $J=8.3,2.5 \mathrm{~Hz}, 1 \mathrm{H}$ ), $7.58-$ $7.49(\mathrm{~m}, 2 \mathrm{H}), 7.45(\mathrm{~d}, J=8.3 \mathrm{~Hz}, 1 \mathrm{H}), 7.42-7.34(\mathrm{~m}, 2 \mathrm{H}), 7.35-7.28(\mathrm{~m}, 1 \mathrm{H}), 7.14$ (d, $J=16.4 \mathrm{~Hz}$, $1 \mathrm{H}, \mathrm{CH}=\mathrm{CH}), 6.99(\mathrm{~d}, J=16.4 \mathrm{~Hz}, 1 \mathrm{H}, \mathrm{CH}=\mathrm{CH})$.

${ }^{13} \mathrm{C}$ NMR (101 MHz, Chloroform- $d$ ) $\delta 148.7(\mathrm{CH}), 140.6(\mathrm{C}), 136.4(\mathrm{C}), 135.1(\mathrm{CH}), 132.5(\mathrm{C}), 131.8$ $(\mathrm{CH}), 129.0(\mathrm{CH}), 128.6(\mathrm{CH}), 128.1(\mathrm{CH}), 126.9(\mathrm{CH}), 123.5(\mathrm{CH})$.

HRMS (ESI-TOF) $\mathrm{m} / z$ : $[\mathrm{M}+\mathrm{H}]^{+}$Calcd for $\mathrm{C}_{13} \mathrm{H}_{11} \mathrm{BrN} 260.0069,262.0049$; Found 260.0068, 262.0039 . 


\section{Synthesis of sulfonium salts}

General procedure C: Sulfide (1.1 equiv.), $\mathrm{Ph}_{2} \operatorname{IOTf}\left(1.0\right.$ equiv.) and $\mathrm{Cu}(\mathrm{OTf})_{2}(5 \mathrm{~mol} \%)$ were added to a crimptop vial and sealed. The vial was evacuated and purged with $\mathrm{N}_{2}$ three times. Dry DCE $(0.6$ M) was added to the vial and the reaction was heated to $95^{\circ} \mathrm{C}$ for $16 \mathrm{~h}$ (the flask sitting in an aluminium block placed on a stirrer hotplate). Then the reaction was allowed to cool and sat. aq. $\mathrm{NH}_{4} \mathrm{Cl}(20 \mathrm{~mL})$ was added. The product was extracted using $\mathrm{CH}_{2} \mathrm{Cl}_{2}(3 \times 20 \mathrm{~mL})$ and washed with $\mathrm{H}_{2} \mathrm{O}(2 \times 20 \mathrm{~mL})$ and brine $(20 \mathrm{~mL})$. The solvent was evaporated and product was isolated by FCC.

Phenyl(pyridin-2-yl)(p-tolyl)sulfonium trifluoromethanesulfonate 2a<smiles>Cc1ccc([Sb](c2ccccc2)c2ccccn2)cc1</smiles>

Product 2a was synthesized via General procedure $\mathrm{C}$ with sulfide $1 \mathrm{a}$ (2.21 g, $11.0 \mathrm{mmol}), \mathrm{Ph}_{2} \mathrm{IOTf}(4.30$ $\mathrm{g}, 10.0 \mathrm{mmol})$ and $\mathrm{Cu}(\mathrm{OTf})_{2}(0.18 \mathrm{~g}, 0.50 \mathrm{mmol})$. Purification by $\mathrm{FCC}\left(5 \% \mathrm{MeOH}\right.$ in $\left.\mathrm{CH}_{2} \mathrm{Cl}_{2}\right)$ gave sulfonium salt 2a as a light brown solid (2.94 $\mathrm{g}, 69 \%)$.

TLC: $R_{\mathrm{f}}=0.47\left(5 \% \mathrm{MeOH}\right.$ in $\left.\mathrm{CH}_{2} \mathrm{Cl}_{2}\right)$.

${ }^{1} \mathrm{H}$ NMR (400 MHz, Chloroform- $d$ ) $\delta 8.74$ (dd, $\left.J=4.7,1.8 \mathrm{~Hz}, 1 \mathrm{H}\right), 8.18$ (d, $\left.J=7.6 \mathrm{~Hz}, 1 \mathrm{H}\right), 8.07$ (td, $J=7.7,1.8 \mathrm{~Hz}, 1 \mathrm{H}), 7.85-7.80(\mathrm{~m}, 2 \mathrm{H}), 7.77(\mathrm{~d}, J=8.4 \mathrm{~Hz}, 2 \mathrm{H}), 7.73-7.66(\mathrm{~m}, 2 \mathrm{H}), 7.66-7.60$ (m, $2 \mathrm{H}), 7.44(\mathrm{~d}, J=8.2 \mathrm{~Hz}, 2 \mathrm{H}), 2.42\left(\mathrm{~s}, 3 \mathrm{H}, \mathrm{CH}_{3}\right)$.

${ }^{13} \mathrm{C}$ NMR (101 MHz, Chloroform-d) $\delta 152.5(\mathrm{CH}), 147.0(\mathrm{C}), 146.6(\mathrm{C}), 140.7(\mathrm{CH}), 134.6(\mathrm{CH}), 132.1$ $(\mathrm{CH}), 131.9(\mathrm{CH}), 131.5(\mathrm{CH}), 131.3(\mathrm{CH}), 129.2(\mathrm{CH}), 128.7(\mathrm{CH}), 124.9(\mathrm{C}), 120.9$ (q, J = 320.9 Hz, OTf), $120.6(\mathrm{C}), 21.7\left(\mathrm{CH}_{3}\right)$.

${ }^{19}$ F NMR (282 MHz, Chloroform- $d$ ) $\delta$-78.2.

HRMS (ESI-TOF) $m / z$ : [M-OTf] ${ }^{+}$Calcd for $\mathrm{C}_{18} \mathrm{H}_{16} \mathrm{NS}$ 278.0998; Found 278.1003. 


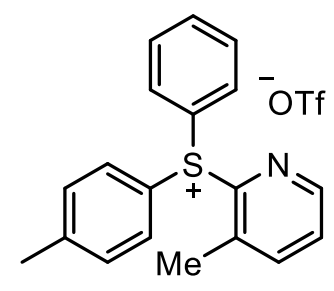

Product $\mathbf{2 b}$ was synthesized via General procedure $\mathrm{C}$ with sulfide $\mathbf{1 b}(1.00 \mathrm{~g}, 4.65 \mathrm{mmol}), \mathrm{Ph}_{2} \mathrm{IOTf}$ $(1.82 \mathrm{~g}, 4.22 \mathrm{mmol})$ and $\mathrm{Cu}(\mathrm{OTf})_{2}(76.3 \mathrm{mg}, 0.21 \mathrm{mmol})$. Purification by $\mathrm{FCC}\left(5 \% \mathrm{MeOH}\right.$ in $\left.\mathrm{CH}_{2} \mathrm{Cl}_{2}\right)$ gave sulfonium salt $\mathbf{2 b}$ as a brown solid (632 $\mathrm{mg}, 34 \%$ ).

TLC: $R_{\mathrm{f}}=0.42\left(5 \% \mathrm{MeOH}\right.$ in $\left.\mathrm{CH}_{2} \mathrm{Cl}_{2}\right)$.

${ }^{1} \mathrm{H}$ NMR (400 MHz, Chloroform- $d$ ) $\delta 8.55$ (ddd, $\left.J=4.6,1.8,0.7 \mathrm{~Hz}, 1 \mathrm{H}\right), 7.94-7.87(\mathrm{~m}, 1 \mathrm{H}), 7.83-$ $7.77(\mathrm{~m}, 2 \mathrm{H}), 7.75-7.69(\mathrm{~m}, 3 \mathrm{H}), 7.68-7.63(\mathrm{~m}, 2 \mathrm{H}), 7.61-7.57(\mathrm{~m}, 1 \mathrm{H}), 7.51-7.41(\mathrm{~m}, 2 \mathrm{H}), 2.67$ (s, 3H), $2.45\left(\mathrm{~s}, 3 \mathrm{H}, \mathrm{CH}_{3}\right)$.

${ }^{13} \mathrm{C}$ NMR (101 MHz, Chloroform-d) $\delta 149.7$ (CH), $146.4(\mathrm{C}), 145.3(\mathrm{C}), 142.1(\mathrm{CH}), 138.8(\mathrm{C}), 134.5$ $(\mathrm{CH}), 132.3(\mathrm{CH}), 132.1(\mathrm{CH}), 132.0(\mathrm{CH}), 131.3(\mathrm{CH}), 128.5(\mathrm{CH}), 124.2(\mathrm{C}), 121.0(\mathrm{q}, J=320.7 \mathrm{~Hz}$, OTf), $119.8(\mathrm{C}), 21.8\left(\mathrm{CH}_{3}\right), 18.6\left(\mathrm{CH}_{3}\right)$.

HRMS (ESI-TOF) $m / z$ : [M-OTf] ${ }^{+}$Calcd for $\mathrm{C}_{19} \mathrm{H}_{18} \mathrm{NS}$ 292.1154; Found 292.1154.

Isoquinolin-1-yl(phenyl)(p-tolyl)sulfonium trifluoromethanesulfonate 2c<smiles>Cc1ccc([Sb](c2ccccc2)c2nccc3ccccc23)cc1</smiles>

Product 2c was synthesized via General procedure $\mathrm{C}$ with sulfide $1 \mathrm{c}$ (1.00 g, $4.06 \mathrm{mmol}), \mathrm{Ph}_{2} \mathrm{IOTf}$ (1.59 $\mathrm{g}, 3.69 \mathrm{mmol})$ and $\mathrm{Cu}(\mathrm{OTf})_{2}(67.0 \mathrm{mg}, 0.185 \mathrm{mmol})$. Purification by $\mathrm{FCC}\left(5 \% \mathrm{MeOH}\right.$ in $\left.\mathrm{CH}_{2} \mathrm{Cl}_{2}\right)$ gave sulfonium salt $\mathbf{2 c}$ as a brown oil (1.04 g, 59\%).

TLC: $R_{\mathrm{f}}=0.49\left(5 \% \mathrm{MeOH}\right.$ in $\left.\mathrm{CH}_{2} \mathrm{Cl}_{2}\right)$.

${ }^{1} \mathrm{H}$ NMR (500 MHz, Chloroform- $d$ ) $\delta 8.83-8.68$ (m, 1H), 8.63 (d, $\left.J=5.4 \mathrm{~Hz}, 1 \mathrm{H}\right), 8.11-8.03$ (m, 2H), $7.97-7.84(\mathrm{~m}, 6 \mathrm{H}), 7.74-7.68(\mathrm{~m}, 1 \mathrm{H}), 7.67-7.57(\mathrm{~m}, 2 \mathrm{H}), 7.50-7.41(\mathrm{~m}, 2 \mathrm{H}), 2.43(\mathrm{~s}, 3 \mathrm{H})$.

${ }^{13} \mathrm{C}$ NMR (126 MHz, Chloroform-d) $\delta 146.5$ (C), $146.0(\mathrm{C}), 143.1(\mathrm{CH}), 138.2(\mathrm{C}), 134.6(\mathrm{CH}), 133.1$ $(\mathrm{CH}), 132.5(\mathrm{CH}), 132.2(\mathrm{CH}), 132.1(\mathrm{CH}), 131.8(\mathrm{CH}), 131.3(\mathrm{CH}), 128.4(\mathrm{C}), 128.1(\mathrm{CH}), 126.6$ $(\mathrm{CH}), 124.3(\mathrm{C}), 124.0(\mathrm{CH}), 120.0(\mathrm{C}), 21.8\left(\mathrm{CH}_{3}\right)$. Signal for OTf not detected. 
HRMS (ESI-TOF) $m / z$ : [M-OTf] ${ }^{+}$Calcd for $\mathrm{C}_{22} \mathrm{H}_{18} \mathrm{NS}$ 328.1154; Found 328.1154.

Phenyl(p-tolyl)(4-(trifluoromethyl)pyridin-2-yl)sulfonium trifluoromethanesulfonate 2d<smiles>Cc1ccc([S+](c2ccccc2)c2cc(C(F)(F)F)ccn2)cc1</smiles>

Product 2d was synthesized via General procedure $\mathrm{C}$ with sulfide $1 \mathbf{d}(500 \mathrm{mg}, 1.86 \mathrm{mmol}), \mathrm{Ph}_{2} \mathrm{IOTf}$ (726 mg, $1.69 \mathrm{mmol})$ and $\mathrm{Cu}(\mathrm{OTf})_{2}(31 \mathrm{mg}, 0.08 \mathrm{mmol})$. Purification by $\mathrm{FCC}\left(5 \% \mathrm{MeOH}\right.$ in $\left.\mathrm{CH}_{2} \mathrm{Cl}_{2}\right)$ gave sulfonium salt $\mathbf{2 d}$ as a yellow oil (443 $\mathrm{mg}, 53 \%)$.

TLC: $R_{\mathrm{f}}=0.49\left(5 \% \mathrm{MeOH}\right.$ in $\left.\mathrm{CH}_{2} \mathrm{Cl}_{2}\right)$.

${ }^{1} \mathrm{H}$ NMR (500 MHz, Chloroform- $d$ ) $\delta 9.04-8.95(\mathrm{~m}, 1 \mathrm{H}), 8.56(\mathrm{dt}, J=1.5,0.7 \mathrm{~Hz}, 1 \mathrm{H}), 7.94-7.88$ (m, 3H), $7.88-7.82(\mathrm{~m}, 2 \mathrm{H}), 7.77-7.69(\mathrm{~m}, 1 \mathrm{H}), 7.69-7.60(\mathrm{~m}, 2 \mathrm{H}), 7.51-7.45(\mathrm{~m}, 2 \mathrm{H}), 2.45(\mathrm{~s}$, $\left.3 \mathrm{H}, \mathrm{CH}_{3}\right)$.

${ }^{13} \mathrm{C}$ NMR (126 MHz, Chloroform- $d$ ) $\delta 153.6(\mathrm{CH}), 149.0(\mathrm{C}), 147.0(\mathrm{C}), 142.3$ (q, $J=36.1 \mathrm{~Hz}, C \mathrm{CF}_{3}$ ), $134.9(\mathrm{CH}), 132.3(\mathrm{CH}), 132.3(\mathrm{CH}), 131.9(\mathrm{CH}), 131.5(\mathrm{CH}), 125.4(\mathrm{q}, J=3.5 \mathrm{~Hz}, \mathrm{CH}), 124.6(\mathrm{q}, J=$ $3.3 \mathrm{~Hz}, \mathrm{CH}), 121.5$ (q, $J=274.6 \mathrm{~Hz}, \mathrm{CF}_{3}$ ), 120.9 (q, $\left.J=320.5 \mathrm{~Hz}, \mathrm{OTf}\right), 120.2$ (C), 119.7 (C), 21.8 $\left(\mathrm{CH}_{3}\right)$.

${ }^{19}$ F NMR (282 MHz, Chloroform- $d$ ) $\delta-64.5,-78.3$.

HRMS (ESI-TOF) $m / z$ : [M-OTf] ${ }^{+}$Calcd for $\mathrm{C}_{19} \mathrm{H}_{15} \mathrm{~F}_{3} \mathrm{NS}$ 346.0872; Found 346.0873.

(5-Nitropyridin-2-yl)(phenyl)(p-tolyl)sulfonium trifluoromethanesulfonate 2e<smiles>Cc1ccc([Sb](c2ccccc2)c2ccc([N+](=O)[O-])cn2)cc1</smiles>

Product 2e was synthesized via General procedure $\mathrm{C}$ with sulfide 1e (1.00 g, $4.06 \mathrm{mmol}), \mathrm{Ph}_{2} \mathrm{IOTf}$ (1.59 $\mathrm{g}, 3.69 \mathrm{mmol})$ and $\mathrm{Cu}(\mathrm{OTf})_{2}(67.0 \mathrm{mg}, 0.185 \mathrm{mmol})$. Purification by $\mathrm{FCC}\left(5 \% \mathrm{MeOH}\right.$ in $\left.\mathrm{CH}_{2} \mathrm{Cl}_{2}\right)$ gave sulfonium salt $2 \mathrm{e}$ as a brown oil $(1.04 \mathrm{~g}, 60 \%)$.

TLC: $R_{\mathrm{f}}=0.35\left(5 \% \mathrm{MeOH}\right.$ in $\left.\mathrm{CH}_{2} \mathrm{Cl}_{2}\right)$. 
${ }^{1} \mathrm{H}$ NMR (500 MHz, Chloroform- $d$ ) $\delta 9.43(\mathrm{dd}, J=2.6,0.6 \mathrm{~Hz}, 1 \mathrm{H}), 8.78(\mathrm{dd}, J=8.7,2.6 \mathrm{~Hz}, 1 \mathrm{H}), 8.47$ (dd, $J=8.6,0.7 \mathrm{~Hz}, 1 \mathrm{H}), 7.95-7.89$ (m, 2H), $7.88-7.82(\mathrm{~m}, 2 \mathrm{H}), 7.80-7.73(\mathrm{~m}, 1 \mathrm{H}), 7.72-7.65$ (m, 2H), $7.53-7.47(\mathrm{~m}, 2 \mathrm{H}), 2.46\left(\mathrm{~s}, 3 \mathrm{H}, \mathrm{CH}_{3}\right)$.

${ }^{13} \mathrm{C}$ NMR (101 MHz, Chloroform- $d$ ) $\delta 152.3,147.4,146.9,146.2,135.8,135.2,132.6,132.5,132.2$, $131.7,130.1,124.2,119.7,21.9\left(\mathrm{CH}_{3}\right)$. Signal for OTf not detected.

HRMS (ESI-TOF) $m / z$ : [M-OTf] ${ }^{+}$Calcd for $\mathrm{C}_{18} \mathrm{H}_{15} \mathrm{~N}_{2} \mathrm{O}_{2} \mathrm{~S} 323.0849$; Found 323.0844.

\section{Phenyl(p-tolyl)(5-(trifluoromethyl)pyridin-2-yl)sulfonium trifluoromethanesulfonate $2 \mathrm{f}$}<smiles>Cc1ccc([Sb](c2ccccc2)c2ccc(C(F)(F)F)cn2)cc1</smiles>

Product $2 \mathbf{f}$ was synthesized via General procedure $\mathrm{C}$ with sulfide $1 \mathbf{f}$ (1.0 g, $3.7 \mathrm{mmol}), \mathrm{Ph}_{2} \mathrm{IOTf}(1.45$ $\mathrm{g}, 3.37 \mathrm{mmol})$ and $\mathrm{Cu}(\mathrm{OTf})_{2}(61.0 \mathrm{mg}, 0.168 \mathrm{mmol})$. Purification by $\mathrm{FCC}\left(5 \% \mathrm{MeOH}\right.$ in $\left.\mathrm{CH}_{2} \mathrm{Cl}_{2}\right)$ gave sulfonium salt $\mathbf{2 f}$ as a brown oil $(1.21 \mathrm{~g}, 72 \%)$.

TLC: $R_{\mathrm{f}}=0.35\left(5 \% \mathrm{MeOH}\right.$ in $\left.\mathrm{CH}_{2} \mathrm{Cl}_{2}\right)$.

${ }^{1} \mathrm{H}$ NMR (400 MHz, Chloroform- $d$ ) $\delta 8.96(\mathrm{dd}, J=2.4,1.0 \mathrm{~Hz}, 1 \mathrm{H}), 8.63(\mathrm{~d}, J=8.3 \mathrm{~Hz}, 1 \mathrm{H}), 8.32$ (dd, $J=8.4,2.4 \mathrm{~Hz}, 1 \mathrm{H}), 7.94-7.88(\mathrm{~m}, 2 \mathrm{H}), 7.87-7.82(\mathrm{~m}, 2 \mathrm{H}), 7.79-7.73(\mathrm{~m}, 1 \mathrm{H}), 7.71-7.65(\mathrm{~m}$, 2H), $7.52-7.44(\mathrm{~m}, 2 \mathrm{H}), 2.47\left(\mathrm{~s}, 3 \mathrm{H}, \mathrm{CH}_{3}\right)$.

${ }^{13} \mathrm{C}$ NMR (101 MHz, Chloroform- $d$ ) $\delta$ 151.1, 148.8 (q, $\left.J=3.9 \mathrm{~Hz}\right), 147.1,138.2$ (q, $\left.J=3.5 \mathrm{~Hz}\right), 135.0$, 132.4, 132.3, 131.9, 131.6, 130.94 (q, $\left.J=34.2 \mathrm{~Hz}, C \mathrm{CF}_{3}\right), 129.9,124.3,122.2$ (q, $\left.J=273.8 \mathrm{~Hz}, \mathrm{CF}_{3}\right)$, 120.9 (q, $J=320.5 \mathrm{~Hz}, \mathrm{OTf}), 119.9,21.9\left(\mathrm{CH}_{3}\right)$.

${ }^{19}$ F NMR (376 MHz, Chloroform- $d$ ) $\delta-62.8,-78.3$.

HRMS (ESI-TOF) $m / z$ : [M-OTf] ${ }^{+}$Calcd for $\mathrm{C}_{19} \mathrm{H}_{15} \mathrm{~F}_{3} \mathrm{NS}$ 346.0872; Found 346.0873. 
<smiles>COc1ccc([SH](c2ccccc2)c2ccc(C)cc2)nc1</smiles>

Product $2 \mathrm{~g}$ was synthesized via General procedure $\mathrm{C}$ with sulfide $1 \mathrm{~g}(0.80 \mathrm{~g}, 3.5 \mathrm{mmol}), \mathrm{Ph}_{2} \mathrm{IOTf}(1.49$ $\mathrm{g}, 3.46 \mathrm{mmol})$ and $\mathrm{Cu}(\mathrm{OTf})_{2}(63 \mathrm{mg}, 0.17 \mathrm{mmol})$. Purification by $\mathrm{FCC}\left(5 \% \mathrm{MeOH}\right.$ in $\left.\mathrm{CH}_{2} \mathrm{Cl}_{2}\right)$ gave sulfonium salt $2 \mathrm{~g}$ as a brown oil (1.30 $\mathrm{g}, 82 \%)$.

TLC: $R_{\mathrm{f}}=0.21\left(5 \% \mathrm{MeOH}\right.$ in $\left.\mathrm{CH}_{2} \mathrm{Cl}_{2}\right)$.

${ }^{1} \mathrm{H}$ NMR (500 MHz, Chloroform- $d$ ) $\delta 8.39(\mathrm{~d}, J=3.2 \mathrm{~Hz}, 1 \mathrm{H}), 8.26(\mathrm{~d}, J=8.8 \mathrm{~Hz}, 1 \mathrm{H}), 7.77(\mathrm{dd}, J=$ 7.6, 1.7 Hz, 2H), 7.73 (d, $J=8.4 \mathrm{~Hz}, 2 \mathrm{H}), 7.71-7.65(\mathrm{~m}, 1 \mathrm{H}), 7.65-7.58(\mathrm{~m}, 2 \mathrm{H}), 7.54$ (dd, $J=8.8$, $3.3 \mathrm{~Hz}, 1 \mathrm{H}), 7.43$ (d, $J=8.3 \mathrm{~Hz}, 2 \mathrm{H}), 3.93$ (s, 3H, OMe), 2.42 (s, 3H, Me).

${ }^{13} \mathrm{C}$ NMR (126 MHz, Chloroform- $d$ ) $\delta 159.6(\mathrm{C}), 146.2(\mathrm{C}), 142.5(\mathrm{CH}), 135.3(\mathrm{C}), 134.3(\mathrm{CH}), 132.0$ $(\mathrm{CH}), 131.5(\mathrm{CH}), 131.4(\mathrm{CH}), 131.2(\mathrm{CH}), 131.0(\mathrm{CH}), 125.9(\mathrm{C}), 122.4(\mathrm{CH}), 121.6(\mathrm{C}), 120.9(\mathrm{q}, J$ $=320.7 \mathrm{~Hz}, \mathrm{OTf}$ ), 56.7 (app. d, $J=3.9 \mathrm{~Hz}, \mathrm{OMe}), 21.7\left(\mathrm{CH}_{3}\right)$.

${ }^{19}$ F NMR (282 MHz, Chloroform- $d$ ) $\delta$-78.2.

HRMS (ESI-TOF) $m / z$ : [M-OTf] ${ }^{+}$Calcd for $\mathrm{C}_{19} \mathrm{H}_{18} \mathrm{NOS}$ 308.1104; Found 308.1094.

(5-(Dimethylamino)pyridin-2-yl)(phenyl)(p-tolyl)sulfonium trifluoromethanesulfonate $2 \mathrm{~h}$<smiles>CC(=O)Nc1ccc([Sb](c2ccccc2)c2ccc(C)cc2)cc1</smiles>

Product $\mathbf{2 h}$ was synthesized via General procedure $\mathrm{C}$ with sulfide $\mathbf{1 h}$ (700 $\mathrm{mg}, 2.86 \mathrm{mmol}), \mathrm{Ph}_{2} \mathrm{IOTf}$ $(1.12 \mathrm{~g}, 2.60 \mathrm{mmol})$ and $\mathrm{Cu}(\mathrm{OTf})_{2}(47.1 \mathrm{mg}, 0.13 \mathrm{mmol})$. Purification by $\mathrm{FCC}\left(5 \% \mathrm{MeOH}\right.$ in $\left.\mathrm{CH}_{2} \mathrm{Cl}_{2}\right)$ gave sulfonium salt $\mathbf{2} \mathbf{h}$ as a yellow oil (1.22 $\mathrm{g}$, quantitative yield).

TLC: $R_{\mathrm{f}}=0.58\left(5 \% \mathrm{MeOH}\right.$ in $\left.\mathrm{CH}_{2} \mathrm{Cl}_{2}\right)$.

${ }^{1} \mathrm{H}$ NMR $(500 \mathrm{MHz}$, Chloroform- $d$ ) $\delta 8.18(\mathrm{~d}, J=3.2 \mathrm{~Hz}, 1 \mathrm{H}), 8.07(\mathrm{~d}, J=9.0 \mathrm{~Hz}, 1 \mathrm{H}), 7.78-7.74(\mathrm{~m}$, 2H), $7.73-7.70(\mathrm{~m}, 2 \mathrm{H}), 7.69-7.64(\mathrm{~m}, 1 \mathrm{H}), 7.63-7.58(\mathrm{~m}, 2 \mathrm{H}), 7.45-7.39$ (m, 2H), 7.12 (dd, $J=$ 9.0, $3.2 \mathrm{~Hz}, 1 \mathrm{H}), 3.11\left(\mathrm{~s}, 6 \mathrm{H}, \mathrm{NMe}_{2}\right), 2.43\left(\mathrm{~s}, 3 \mathrm{H}, \mathrm{CH}_{3}\right)$. 
${ }^{13} \mathrm{C}$ NMR (126 MHz, Chloroform- $d$ ) $\delta 152.3(\mathrm{C}), 148.7(\mathrm{C}), 145.6(\mathrm{C}), 137.2(\mathrm{CH}), 133.9(\mathrm{CH}), 131.8$ $(\mathrm{CH}), 131.2(\mathrm{CH}), 131.0(\mathrm{CH}), 130.8(\mathrm{CH}), 127.3(\mathrm{C}), 123.1$ (C), 121.09 (q, $J=321.0 \mathrm{~Hz}, \mathrm{OTf}), 119.0$ $(\mathrm{CH}), 40.0\left(\mathrm{NMe}_{2}\right), 21.8\left(\mathrm{CH}_{3}\right)$.

HRMS (ESI-TOF) $m / z$ : [M-OTf] $]^{+}$Calcd for $\mathrm{C}_{20} \mathrm{H}_{21} \mathrm{~N}_{2} \mathrm{~S} 321.1420$; Found 321.1422 .

(6-Bromopyridin-2-yl)(phenyl)(p-tolyl)sulfonium trifluoromethanesulfonate $2 \mathrm{i}$<smiles>Cc1ccc([Sb](c2ccccc2)c2cccc(Br)n2)cc1</smiles>

Product $2 \mathbf{i}$ was synthesized via General procedure $\mathrm{C}$ with sulfide $1 \mathbf{i}(0.77 \mathrm{~g}, 2.7 \mathrm{mmol}), \mathrm{Ph}_{2} \mathrm{IOTf}(1.13$ $\mathrm{g}, 2.61 \mathrm{mmol})$ and $\mathrm{Cu}(\mathrm{OTf})_{2}(47 \mathrm{mg}, 0.13 \mathrm{mmol})$. Purification by $\mathrm{FCC}\left(5 \% \mathrm{MeOH}\right.$ in $\left.\mathrm{CH}_{2} \mathrm{Cl}_{2}\right)$ gave sulfonium salt $2 \mathbf{i}$ as a brown oil (1.05 g, 79\%).

TLC: $R_{\mathrm{f}}=0.27\left(5 \% \mathrm{MeOH}\right.$ in $\left.\mathrm{CH}_{2} \mathrm{Cl}_{2}\right)$.

${ }^{1} \mathrm{H}$ NMR (400 MHz, Chloroform- $d$ ) $\delta 8.24(\mathrm{~d}, J=7.7 \mathrm{~Hz}, 1 \mathrm{H}), 7.98(\mathrm{t}, J=7.8 \mathrm{~Hz}, 1 \mathrm{H}), 7.84$ (dd, $J=$ $7.2,1.7 \mathrm{~Hz}, 2 \mathrm{H}), 7.82-7.76(\mathrm{~m}, 3 \mathrm{H}), 7.76-7.71(\mathrm{~m}, 1 \mathrm{H}), 7.69-7.62(\mathrm{~m}, 2 \mathrm{H}), 7.47(\mathrm{~d}, J=8.3 \mathrm{~Hz}$, $2 \mathrm{H}), 2.44\left(\mathrm{~s}, 3 \mathrm{H}, \mathrm{CH}_{3}\right)$.

${ }^{13} \mathrm{C}$ NMR (126 MHz, Chloroform- $d$ ) $\delta 146.9$ (C), 146.5 (C), 144.0 (C), $142.6(\mathrm{CH}), 134.9(\mathrm{CH}), 133.5$ $(\mathrm{CH}), 132.3(\mathrm{CH}), 131.9(\mathrm{CH}), 131.5(\mathrm{CH}), 128.4(\mathrm{CH}), 124.5(\mathrm{C}), 120.9$ (q, J=320.7 Hz, OTf), 120.1 (C), $21.8\left(\mathrm{CH}_{3}\right)$.

${ }^{19}$ F NMR (282 MHz, Chloroform- $d$ ) $\delta$-78.2.

HRMS (ESI-TOF) $m / z$ : [M-OTf] ${ }^{+}$Calcd for $\mathrm{C}_{18} \mathrm{H}_{15} \mathrm{BrNS}$ 356.0103, 358.0083; Found 356.0119, 358.0123 . 


\section{Synthesis of bipyridines}

General procedure D: Halopyridine ( $0.45 \mathrm{mmol}, 1.5$ equiv.) was added to an oven-dried crimptop vial and sealed. The vial was evacuated and purged with $\mathrm{N}_{2}$ three times. The halopyridine was dissolved in dry THF $(1.5 \mathrm{~mL})$ and cooled to $-78^{\circ} \mathrm{C} . n$-BuLi $(0.19 \mathrm{~mL}, 0.45 \mathrm{mmol}, 1.5$ equiv., $2.4 \mathrm{M}$ in hexanes) was added dropwise to the stirring solution over $2 \mathrm{~min}$. The reaction was allowed to stir for $30 \mathrm{~min}$ at $78^{\circ} \mathrm{C}$. Sulfonium salt $(0.3 \mathrm{mmol}, 1.0$ equiv. $)$ was added to a separate oven-dried crimptop vial and dissolved in dry THF $(1.5 \mathrm{~mL})$. The solution of sulfonium salt was added dropwise down the side of the vial to the lithiated pyridine solution over 2 min. The reaction was allowed to stir for $2 \mathrm{~h}$ at $-78{ }^{\circ} \mathrm{C}$. Sat. aq. $\mathrm{NH}_{4} \mathrm{Cl}(3 \mathrm{~mL})$ was added slowly to quench any excess organolithium. The product was extracted with EtOAc $(3 \times 10 \mathrm{~mL})$, the combined organic layers were washed with $\mathrm{H}_{2} \mathrm{O}(20 \mathrm{~mL})$ and brine $(20 \mathrm{~mL})$. The solvent was evaporated and the product was isolated by FCC.

\section{2,3-Bipyridine 3}

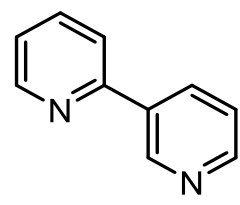

Product 3 was synthesized via General procedure D using 3-iodopyridine ( $92.3 \mathrm{mg}, 0.45 \mathrm{mmol})$ and sulfonium salt 2a (128.2 mg, $0.3 \mathrm{mmol})$. Purification by FCC ( $80 \% \mathrm{Et}_{2} \mathrm{O}$ in pentane) gave bipyridine 3 as a colourless oil $(42.1 \mathrm{mg}, 90 \%)$.

TLC: $R_{\mathrm{f}}=0.13\left(80 \% \mathrm{Et}_{2} \mathrm{O}\right.$ in pentane).

${ }^{1} \mathrm{H}$ NMR (400 MHz, Chloroform- $\left.d\right) \delta 9.20(\mathrm{~d}, J=2.3 \mathrm{~Hz}, 1 \mathrm{H}), 8.76-8.70(\mathrm{~m}, 1 \mathrm{H}), 8.66(\mathrm{dd}, J=4.9$, $1.7 \mathrm{~Hz}, 1 \mathrm{H}), 8.33$ (dt, $J=8.1,2.0 \mathrm{~Hz}, 1 \mathrm{H}), 7.85-7.72$ (m, 2H), 7.41 (dd, J= 7.9, 4.9 Hz, 1H), 7.33 $7.26(\mathrm{~m}, 1 \mathrm{H})$.

${ }^{13} \mathrm{C}$ NMR (101 MHz, Chloroform- $d$ ) $\delta 154.9,150.2,150.0,148.3,137.1,135.0,134.5,123.7,123.0$, 120.7 .

Spectra were consistent with literature data. ${ }^{7}$

\section{2,2'-Bipyridine 4}<smiles>c1ccc(-c2ccccn2)nc1</smiles>

Product 4 was synthesized via General procedure D using 2-bromopyridine ( $42.9 \mu \mathrm{L}, 0.45 \mathrm{mmol})$ and sulfonium salt $\mathbf{2 a}$ (128.2 $\mathrm{mg}, 0.3 \mathrm{mmol})$. Purification by $\mathrm{FCC}\left(80 \% \mathrm{Et}_{2} \mathrm{O}\right.$ in pentane) gave bipyridine 4 as a white solid $(28.1 \mathrm{mg}, 60 \%)$. 
${ }^{1} \mathrm{H}$ NMR (400 MHz, Chloroform-d) $\delta 8.74-8.61(\mathrm{~m}, 2 \mathrm{H}), 8.40(\mathrm{~d}, J=8.0 \mathrm{~Hz}, 2 \mathrm{H}), 7.82(\mathrm{td}, J=7.7$, $1.8 \mathrm{~Hz}, 2 \mathrm{H}), 7.31$ (ddd, $J=7.6,4.8,1.2 \mathrm{~Hz}, 2 \mathrm{H})$.

${ }^{13} \mathrm{C}$ NMR (101 MHz, Chloroform- $d$ ) $\delta$ 156.3, 149.4, 137.1, 123.9, 121.2.

Spectra are consistent with literature data. ${ }^{8}$

1-(Pyridin-2-yl)isoquinoline 5<smiles>c1ccc(-c2nccc3ccccc23)nc1</smiles>

Product 5 was synthesized via General procedure D using 1-iodoisoquinoline (114.8 mg, $0.45 \mathrm{mmol}$ ) and sulfonium salt $\mathbf{2 a}(128.2 \mathrm{mg}, 0.3 \mathrm{mmol})$. Purification by $\mathrm{FCC}\left(80 \% \mathrm{Et}_{2} \mathrm{O}\right.$ in pentane) gave bipyridine 5 as a yellow oil (39.9 mg, 64\%).

${ }^{1} \mathrm{H}$ NMR (500 MHz, Chloroform- $d$ ) $\delta 8.80(\mathrm{~d}, J=1.6 \mathrm{~Hz}, 1 \mathrm{H}), 8.68-8.55(\mathrm{~m}, 2 \mathrm{H}), 8.00$ (dt, $J=7.9$, $1.1 \mathrm{~Hz}, 1 \mathrm{H}), 7.95-7.84(\mathrm{~m}, 2 \mathrm{H}), 7.75-7.66(\mathrm{~m}, 2 \mathrm{H}), 7.60$ (ddd, $J=8.3,6.8,1.3 \mathrm{~Hz}, 1 \mathrm{H}), 7.41$ (ddd, $J=7.6,4.8,1.3 \mathrm{~Hz}, 1 \mathrm{H})$.

${ }^{13} \mathrm{C}$ NMR (101 MHz, Chloroform-d) $\delta 158.5,157.8,148.8,142.1,137.3,137.1,130.2,127.9,127.8$, $127.0,126.8,125.4,123.4,121.4$.

Spectra are consistent with literature data. ${ }^{9}$

\section{3-Methyl-2,2'-bipyridine 6}<smiles>Cc1cccnc1-c1ccccn1</smiles>

Product 6 was synthesized via General procedure D using 2-bromo-3-methylpyridine (50 $\mu \mathrm{L}, 0.45$ $\mathrm{mmol})$ and sulfonium salt $\mathbf{2 a}(128.2 \mathrm{mg}, 0.3 \mathrm{mmol})$. Purification by $\mathrm{FCC}\left(70 \% \mathrm{Et}_{2} \mathrm{O}\right.$ in pentane) gave bipyridine 6 as a yellow oil (41.2 $\mathrm{mg}, 81 \%)$.

${ }^{1} \mathrm{H}$ NMR (500 MHz, Chloroform- $d$ ) $\delta 8.68(\mathrm{dt}, J=4.9,1.4 \mathrm{~Hz}, 1 \mathrm{H}), 8.53(\mathrm{dd}, J=4.6,1.6 \mathrm{~Hz}, 1 \mathrm{H}), 7.85$ $-7.77(\mathrm{~m}, 2 \mathrm{H}), 7.61(\mathrm{ddd}, J=7.7,1.7,0.8 \mathrm{~Hz}, 1 \mathrm{H}), 7.29$ (ddd, $J=6.7,4.8,1.7 \mathrm{~Hz}, 1 \mathrm{H}), 7.22(\mathrm{dd}, J=$ 7.7, $4.7 \mathrm{~Hz}, 1 \mathrm{H}), 2.51\left(\mathrm{~s}, 3 \mathrm{H}, \mathrm{CH}_{3}\right)$.

${ }^{13} \mathrm{C}$ NMR (126 MHz, Chloroform-d) $\delta 159.1,156.5,148.6,146.9,139.3,136.7,132.4,124.3,123.2$, 122.8, $20.1\left(\mathrm{CH}_{3}\right)$.

Spectra are consistent with literature data. ${ }^{10}$ 


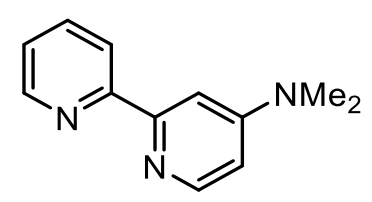

Product 7 was synthesized via General procedure D using 2-bromo- $N, N$-dimethylpyridin-4-amine ( 90.5 $\mathrm{mg}, 0.45 \mathrm{mmol})$ and sulfonium salt $\mathbf{2 a}(128.2 \mathrm{mg}, 0.3 \mathrm{mmol})$. Purification by FCC (2\% $\mathrm{Et}_{3} \mathrm{~N}$ in $\left.\mathrm{Et}_{2} \mathrm{O}\right)$ gave bipyridine 7 as a yellow oil $(30.6 \mathrm{mg}, 51 \%)$.

${ }^{1} \mathrm{H}$ NMR (500 MHz, Chloroform- $d$ ) $\delta 8.66$ (ddd, $\left.J=4.8,1.8,0.9 \mathrm{~Hz}, 1 \mathrm{H}\right), 8.38(\mathrm{dt}, J=8.0,1.1 \mathrm{~Hz}, 1 \mathrm{H})$, $8.32(\mathrm{dd}, J=5.9,0.5 \mathrm{~Hz}, 1 \mathrm{H}), 7.79(\mathrm{ddd}, J=8.0,7.5,1.8 \mathrm{~Hz}, 1 \mathrm{H}), 7.70(\mathrm{~d}, J=2.7 \mathrm{~Hz}, 1 \mathrm{H}), 7.29-7.26$ $(\mathrm{m}, 1 \mathrm{H}), 6.54(\mathrm{dd}, J=5.9,2.7 \mathrm{~Hz}, 1 \mathrm{H}), 3.10\left(\mathrm{~s}, 6 \mathrm{H}, \mathrm{NMe}_{2}\right)$.

${ }^{13} \mathrm{C}$ NMR (126 MHz, Chloroform- $d$ ) $\delta 157.1,156.3,155.4$, 149.5, 149.0, 136.9, 123.5, 121.4, 106.8, 104.0, $39.5\left(\mathrm{NMe}_{2}\right)$.

Spectra are consistent with literature data. ${ }^{11}$

\section{5-Fluoro-2,2'-bipyridine 8}

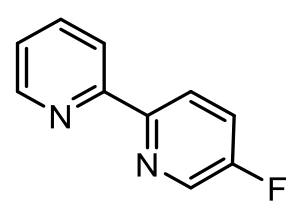

Product 8 was synthesized via General procedure D using 2-bromo-5-fluoropyridine (79.2 mg, 0.45 $\mathrm{mmol})$ and sulfonium salt $2 \mathrm{a}(128.2 \mathrm{mg}, 0.3 \mathrm{mmol})$. Purification by $\mathrm{FCC}\left(80 \% \mathrm{Et}_{2} \mathrm{O}\right.$ in pentane) gave bipyridine 8 as an orange oil (12.4 mg, 24\%).

TLC: $R_{\mathrm{f}}=0.38\left(80 \% \mathrm{Et}_{2} \mathrm{O}\right.$ in pentane $)$.

${ }^{1} \mathrm{H}$ NMR (500 MHz, Chloroform- $d$ ) $\delta 8.77$ (ddd, $\left.J=4.8,1.9,1.0 \mathrm{~Hz}, 1 \mathrm{H}\right), 8.61-8.56(\mathrm{~m}, 1 \mathrm{H}), 8.53(\mathrm{~d}$, $J=5.0 \mathrm{~Hz}, 1 \mathrm{H}), 8.00(\mathrm{dd}, J=6.8,5.0 \mathrm{~Hz}, 1 \mathrm{H}), 7.91(\mathrm{ddt}, J=7.9,1.9,1.1 \mathrm{~Hz}, 1 \mathrm{H}), 7.82(\mathrm{td}, J=7.8,1.9$ $\mathrm{Hz}, 1 \mathrm{H}), 7.36$ (ddd, $J=7.5,4.8,1.1 \mathrm{~Hz}, 1 \mathrm{H})$.

${ }^{13} \mathrm{C}$ NMR (126 MHz, Chloroform- $d$ ) $\delta 157.2(\mathrm{~d}, J=256.8 \mathrm{~Hz}, \mathrm{CF}), 150.7(\mathrm{~d}, J=2.4 \mathrm{~Hz}, \mathrm{C}), 150.3$ (CH), 146.3 (d, $J=5.2 \mathrm{~Hz}, \mathrm{CH}), 139.4$ (d, $J=26.3 \mathrm{~Hz}, \mathrm{CH}), 136.9(\mathrm{CH}), 134.1$ (d, $J=9.0 \mathrm{~Hz}, \mathrm{C}), 125.2$ (d, $J=10.0 \mathrm{~Hz}, \mathrm{CH}), 124.2(\mathrm{CH}), 124.1(\mathrm{CH})$.

${ }^{19}$ F NMR (282 MHz, Chloroform- $d$ ) $\delta-131.7(\mathrm{~d}, J=5.3 \mathrm{~Hz})$.

HRMS (ESI-TOF) $m / z$ : [M+H] $]^{+}$Calcd for $\mathrm{C}_{10} \mathrm{H}_{8} \mathrm{FN}_{2}$ 175.0666; Found 175.0667. 


\section{$N, N$-Dimethyl-[2,2'-bipyridin]-5-amine 9}

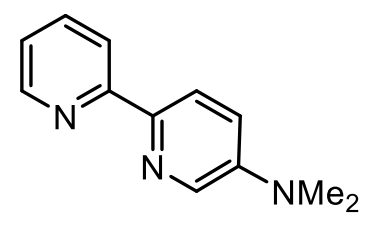

Product 9 was synthesized via General procedure D using 6-bromo- $N, N$-dimethylpyridin-3-amine ( 90.5 $\mathrm{mg}, 0.45 \mathrm{mmol})$ and sulfonium salt $2 \mathrm{a}(128.2 \mathrm{mg}, 0.3 \mathrm{mmol})$. Purification by FCC $\left(40 \% \mathrm{Et}_{2} \mathrm{O}\right.$ in pentane) gave bipyridine 9 as an off-white oil (18.5 mg, 31\%).

TLC: $R_{\mathrm{f}}=0.10\left(40 \% \mathrm{Et}_{2} \mathrm{O}\right.$ in pentane $)$.

${ }^{1} \mathrm{H}$ NMR (500 MHz, Chloroform- $d$ ) $\delta 8.60$ (ddd, $\left.J=4.8,1.9,0.9 \mathrm{~Hz}, 1 \mathrm{H}\right), 8.25$ (ddd, $J=9.8,8.5,0.8$ $\mathrm{Hz}, 2 \mathrm{H}), 8.19$ (d, $J=3.0 \mathrm{~Hz}, 1 \mathrm{H}), 7.74(\mathrm{td}, J=7.7,1.8 \mathrm{~Hz}, 1 \mathrm{H}), 7.18$ (ddd, $J=7.4,4.8,1.2 \mathrm{~Hz}, 1 \mathrm{H})$, $7.08(\mathrm{dd}, J=8.9,3.1 \mathrm{~Hz}, 1 \mathrm{H}), 3.05\left(\mathrm{~s}, 6 \mathrm{H}, \mathrm{NMe}_{2}\right)$.

${ }^{13} \mathrm{C}$ NMR (126 MHz, Chloroform- $d$ ) $\delta 156.9$ (C), $149.1(\mathrm{CH}), 146.3(\mathrm{C}), 144.6(\mathrm{C}), 136.8(\mathrm{CH}), 134.0$ $(\mathrm{CH}), 122.2(\mathrm{CH}), 121.4(\mathrm{CH}), 119.8(\mathrm{CH}), 119.1(\mathrm{CH}), 40.2\left(\mathrm{NMe}_{2}\right)$.

HRMS (ESI-TOF) $m / z$ : [M+H] $]^{+}$Calcd for $\mathrm{C}_{12} \mathrm{H}_{14} \mathrm{~N}_{3}$ 200.1182; Found 200.1183.

\section{5-(1,3-Dioxolan-2-yl)-2,2'-bipyridine 10}

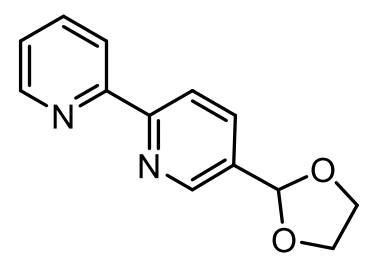

Product 10 was synthesized via General procedure D using 2-bromo-5-(1,3-dioxolan-2-yl)pyridine (103 $\mathrm{mg}, 0.45 \mathrm{mmol}$ ) and sulfonium salt $\mathbf{2 a}(128.2 \mathrm{mg}, 0.3 \mathrm{mmol})$. Purification by FCC ( $40 \% \mathrm{Et}_{2} \mathrm{O}$ in pentane with $1 \% \mathrm{Et}_{3} \mathrm{~N}$ ) gave bipyridine $\mathbf{1 0}$ as an off-white solid $(54.7 \mathrm{mg}, 80 \%)$.

${ }^{1} \mathrm{H}$ NMR (400 MHz, Chloroform- $d$ ) $\delta 8.76(\mathrm{~d}, J=2.2 \mathrm{~Hz}, 1 \mathrm{H}), 8.72-8.61(\mathrm{~m}, 1 \mathrm{H}), 8.43-8.34$ (m, 2H), $7.92(\mathrm{dd}, J=8.2,2.2 \mathrm{~Hz}, 1 \mathrm{H}), 7.81(\mathrm{td}, J=7.7,1.8 \mathrm{~Hz}, 1 \mathrm{H}), 7.31$ (ddd, $J=7.5,4.8,1.2 \mathrm{~Hz}, 1 \mathrm{H})$, $5.93(\mathrm{~s}, 1 \mathrm{H}), 4.16-4.12(\mathrm{~m}, 2 \mathrm{H}), 4.09-4.04(\mathrm{~m}, 2 \mathrm{H})$.

${ }^{13} \mathrm{C}$ NMR (101 MHz, Chloroform-d) $\delta 157.1,156.0,149.4,147.9,137.1,135.3,133.7,124.0,121.4$, $120.8,102.1,65.5$.

Spectra are consistent with literature data. ${ }^{12}$ 


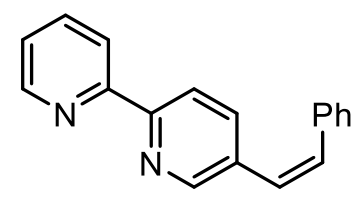

Product 11 was synthesized via General procedure D using (Z)-2-bromo-5-styrylpyridine (117.0 mg, $0.45 \mathrm{mmol})$ and sulfonium salt $\mathbf{2 a}(128.2 \mathrm{mg}, 0.3 \mathrm{mmol})$. Purification by FCC (30\% $\mathrm{Et}_{2} \mathrm{O}$ in pentane) gave bipyridine $\mathbf{1 1}$ as a yellow oil (39.1 $\mathrm{mg}, 50 \%)$.

TLC: $R_{\mathrm{f}}=0.14\left(30 \% \mathrm{Et}_{2} \mathrm{O}\right.$ in pentane).

${ }^{1} \mathrm{H}$ NMR (500 MHz, Chloroform- $d$ ) $\delta 8.66$ (ddd, $\left.J=4.8,1.8,0.9 \mathrm{~Hz}, 1 \mathrm{H}\right), 8.55(\mathrm{~d}, J=2.2 \mathrm{~Hz}, 1 \mathrm{H}), 8.35$ $(\mathrm{dt}, J=8.0,1.1 \mathrm{~Hz}, 1 \mathrm{H}), 8.23(\mathrm{~d}, J=8.2 \mathrm{~Hz}, 1 \mathrm{H}), 7.79(\mathrm{td}, J=7.7,1.8 \mathrm{~Hz}, 1 \mathrm{H}), 7.66(\mathrm{dd}, J=8.3,2.2$ $\mathrm{Hz}, 1 \mathrm{H}), 7.34-7.20(\mathrm{~m}, 6 \mathrm{H}), 6.78(\mathrm{~d}, J=12.2 \mathrm{~Hz}, 1 \mathrm{H}, \mathrm{CH}=\mathrm{CH}), 6.60(\mathrm{~d}, J=12.2 \mathrm{~Hz}, 1 \mathrm{H}, \mathrm{CH}=\mathrm{CH})$.

${ }^{13} \mathrm{C}$ NMR (126 MHz, Chloroform-d) $\delta 156.1(\mathrm{C}), 154.6(\mathrm{C}), 149.8(\mathrm{CH}), 149.3(\mathrm{CH}), 137.0(\mathrm{CH}), 136.9$ (C), $136.7(\mathrm{C}), 133.2(\mathrm{HC}=\mathrm{CH}), 133.0(\mathrm{CH}), 128.9(\mathrm{CH}), 128.7(\mathrm{CH}), 127.8(\mathrm{CH}), 126.5(\mathrm{HC}=\mathrm{CH})$, $123.7(\mathrm{CH}), 121.2(\mathrm{CH}), 120.5(\mathrm{CH})$.

HRMS (ESI-TOF) $m / z$ : [M+H] $]^{+}$Calcd for $\mathrm{C}_{18} \mathrm{H}_{15} \mathrm{~N}_{2}$ 259.1230; Found 259.1231.

5-((Trimethylsilyl)ethynyl)-2,2'-bipyridine 12

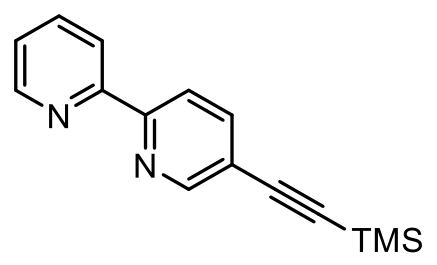

Product 12 was synthesized via General procedure D using 2-bromo-5-((trimethylsilyl)ethynyl)pyridine (114.4 mg, $0.45 \mathrm{mmol})$ and sulfonium salt $2 \mathrm{a}(128.2 \mathrm{mg}, 0.3 \mathrm{mmol})$. Purification by FCC (30\% $\mathrm{Et}_{2} \mathrm{O}$ in pentane) gave bipyridine 12 as a yellow oil (38.8 $\mathrm{mg}, 51 \%)$.

${ }^{1} \mathrm{H}$ NMR $(500 \mathrm{MHz}$, Chloroform- $d$ ) $\delta 8.75-8.71(\mathrm{~m}, 1 \mathrm{H}), 8.67(\mathrm{~d}, J=4.7 \mathrm{~Hz}, 1 \mathrm{H}), 8.39(\mathrm{~d}, J=8.0 \mathrm{~Hz}$, $1 \mathrm{H}), 8.36(\mathrm{~d}, J=8.2 \mathrm{~Hz}, 1 \mathrm{H}), 7.86(\mathrm{dd}, J=8.2,2.1 \mathrm{~Hz}, 1 \mathrm{H}), 7.81(\mathrm{td}, J=7.8,1.8 \mathrm{~Hz}, 1 \mathrm{H}), 7.30$ (ddd, $J=7.5,4.8,1.2 \mathrm{~Hz}, 1 \mathrm{H}), 0.28(\mathrm{~s}, 9 \mathrm{H})$.

${ }^{13} \mathrm{C}$ NMR (101 MHz, Chloroform- $d$ ) $\delta$ 155.6, 155.1, 152.2, 149.4, 139.9, 137.1, 124.1, 121.6, 120.3, $102.0(\mathrm{C} \equiv \mathrm{C}), 99.3(\mathrm{C} \equiv \mathrm{C}),-0.01\left(\mathrm{SiMe}_{3}\right)$.

Spectra are consistent with literature data. ${ }^{3}$ 


\section{6-Bromo-4-methoxy-2,2'-bipyridine 13}<smiles>COc1cc(Br)nc(-c2ccccn2)c1</smiles>

Product 13 was synthesized via General procedure D at $-40{ }^{\circ} \mathrm{C}$ using 2,6-dibromo-4-methoxypyridine $(39.8 \mathrm{mg}, 0.15 \mathrm{mmol})$ and sulfonium salt $\mathbf{2 a}(42.7 \mathrm{mg}, 0.1 \mathrm{mmol})$. Purification by $\mathrm{FCC}\left(50 \% \mathrm{Et}_{2} \mathrm{O}\right.$ in pentane) gave bipyridine $\mathbf{1 3}$ as a white solid (5.8 $\mathrm{mg}, 22 \%$ ).

TLC: $R_{\mathrm{f}}=0.50\left(50 \% \mathrm{Et}_{2} \mathrm{O}\right.$ in pentane $)$.

${ }^{1} \mathrm{H}$ NMR $(300 \mathrm{MHz}$, Chloroform- $d$ ) $\delta 8.65(\mathrm{~d}, J=4.4 \mathrm{~Hz}, 1 \mathrm{H}), 8.40$ (d, $J=8.0 \mathrm{~Hz}, 1 \mathrm{H}), 7.95(\mathrm{~d}, J=1.9$ $\mathrm{Hz}, 1 \mathrm{H}), 7.81(\mathrm{~m}, 1 \mathrm{H}), 7.32$ (dd, $J=7.4,4.8 \mathrm{~Hz}, 1 \mathrm{H}), 7.03$ (d, $J=2.3 \mathrm{~Hz}, 1 \mathrm{H}), 3.95$ (s, 3H).

${ }^{13} \mathrm{C}$ NMR (126 MHz, Chloroform- $d$ ) $\delta 167.9,158.4,154.6,149.2,142.4,137.1,124.5,121.8,114.2$, 106.2, 56.0.

Spectra were consistent with literature data. ${ }^{13}$

\section{6-Methoxy-2,2'-bipyridine 14}

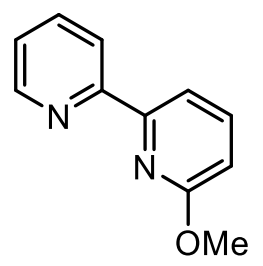

Product 14 was synthesized via General procedure D using 2-bromo-6-methoxypyridine (84.6 mg, 0.45 mmol) and sulfonium salt $2 \mathrm{a}(128.2 \mathrm{mg}, 0.3 \mathrm{mmol})$. Purification by $\mathrm{FCC}\left(5 \% \mathrm{Et}_{2} \mathrm{O}\right.$ in $\left.\mathrm{CH}_{2} \mathrm{Cl}_{2}\right)$ gave bipyridine 14 a colourless oil (30.3 mg, 54\%).

TLC: $R_{\mathrm{f}}=0.12\left(5 \% \mathrm{Et}_{2} \mathrm{O}\right.$ in $\left.\mathrm{CH}_{2} \mathrm{Cl}_{2}\right)$.

${ }^{1} \mathrm{H}$ NMR (400 MHz, Chloroform- $d$ ) $\delta 8.67-8.64(\mathrm{~m}, 1 \mathrm{H}), 8.41(\mathrm{dt}, J=7.9,1.2 \mathrm{~Hz}, 1 \mathrm{H}), 8.01(\mathrm{dd}, J=$ 7.4, $0.6 \mathrm{~Hz} 1 \mathrm{H}), 7.79$ (td, $J=7.7,1.8 \mathrm{~Hz}, 1 \mathrm{H}), 7.70$ (dd, $J=8.1,7.5 \mathrm{~Hz}, 1 \mathrm{H}), 7.28$ (ddd, $J=7.6,4.9$, $1.3 \mathrm{~Hz}, 1 \mathrm{H}), 6.78(\mathrm{dd}, J=8.2,0.6 \mathrm{~Hz}, 1 \mathrm{H}), 4.04(\mathrm{~s}, 3 \mathrm{H})$.

${ }^{13} \mathrm{C}$ NMR (101 MHz, Chloroform-d) $\delta 163.7,156.2,153.6,149.2,139.5,136.9,123.6,121.1,113.8$, 111.2, 53.3.

Spectra were consistent with literature data. ${ }^{14}$ 


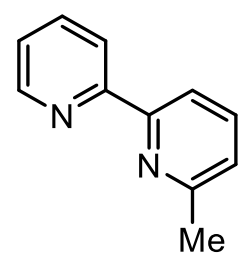

Product 15 was synthesized via General procedure D using 2-bromo-6-methylpyridine (77.4 mg, 0.45 $\mathrm{mmol})$ and sulfonium salt $2 \mathbf{2 a}(128.2 \mathrm{mg}, 0.3 \mathrm{mmol})$. Purification by $\mathrm{FCC}\left(40 \% \mathrm{Et}_{2} \mathrm{O}\right.$ in pentane) gave bipyridine 15 as a yellow oil $(35.7 \mathrm{mg}, 70 \%)$.

${ }^{1} \mathrm{H}$ NMR (500 MHz, Chloroform- $d$ ) $\delta 8.67$ (ddd, $\left.J=4.8,1.9,0.9 \mathrm{~Hz}, 1 \mathrm{H}\right), 8.40(\mathrm{dt}, J=8.0,1.1 \mathrm{~Hz}, 1 \mathrm{H})$, $8.22-8.11(\mathrm{~m}, 1 \mathrm{H}), 7.79(\mathrm{td}, J=7.7,1.8 \mathrm{~Hz}, 1 \mathrm{H}), 7.69(\mathrm{t}, J=7.7 \mathrm{~Hz}, 1 \mathrm{H}), 7.28(\mathrm{ddd}, J=7.5,4.8,1.2$ $\mathrm{Hz}, 1 \mathrm{H}), 7.18-7.12(\mathrm{~m}, 1 \mathrm{H}), 2.63(\mathrm{~s}, 3 \mathrm{H})$.

${ }^{13} \mathrm{C}$ NMR (101 MHz, Chloroform- $d$ ) $\delta 158.1,156.7,155.7,149.3,137.2,137.00,123.6,123.4,121.3$, $118.2,24.8$.

Spectra are consistent with literature data. ${ }^{15}$

6-(p-Tolylthio)-2,2'-bipyridine 16

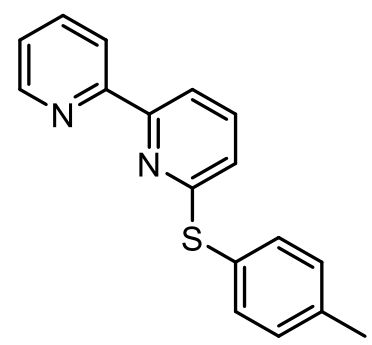

Product 16 was synthesized via General procedure D using 2-bromo-6-( $p$-tolylthio)pyridine 1i (126.1 $\mathrm{mg}, 0.45 \mathrm{mmol})$ and sulfonium salt $2 \mathrm{a}(128.2 \mathrm{mg}, 0.3 \mathrm{mmol})$. Purification by $\mathrm{FCC}\left(30 \% \mathrm{Et}_{2} \mathrm{O}\right.$ in pentane) gave bipyridine 16 as a white solid (45.0 mg, 54\%).

TLC: $R_{\mathrm{f}}=0.40\left(30 \% \mathrm{Et}_{2} \mathrm{O}\right.$ in pentane $)$.

${ }^{1} \mathrm{H}$ NMR (500 MHz, Chloroform- $d$ ) $\delta 8.66-8.64(\mathrm{~m}, 1 \mathrm{H}), 8.35-8.31(\mathrm{~m}, 1 \mathrm{H}), 8.11(\mathrm{~d}, J=7.8 \mathrm{~Hz}$, $1 \mathrm{H}), 7.78(\mathrm{td}, J=7.7,1.7 \mathrm{~Hz}, 1 \mathrm{H}), 7.60-7.55(\mathrm{~m}, 3 \mathrm{H}), 7.32-7.25(\mathrm{~m}, 3 \mathrm{H}), 6.88(\mathrm{~d}, J=8.0 \mathrm{~Hz}, 1 \mathrm{H})$, $2.43\left(\mathrm{~s}, 3 \mathrm{H}, \mathrm{CH}_{3}\right)$.

${ }^{13} \mathrm{C}$ NMR (126 MHz, Chloroform- $d$ ) $\delta 161.6(\mathrm{C}), 155.9$ (C), 155.7 (C), $149.2(\mathrm{CH}), 139.6(\mathrm{C}), 137.6$ $(\mathrm{CH}), 137.0(\mathrm{CH}), 135.6(\mathrm{CH}), 130.5(\mathrm{CH}), 127.4(\mathrm{C}), 123.9(\mathrm{CH}), 121.5(\mathrm{CH}), 120.9(\mathrm{CH}), 117.0$ $(\mathrm{CH}), 21.5\left(\mathrm{CH}_{3}\right)$.

HRMS (ESI-TOF) $m / z$ : [M+H] $]^{+}$Calcd for $\mathrm{C}_{17} \mathrm{H}_{15} \mathrm{~N}_{2} \mathrm{~S} 279.0950$; Found 279.0944 . 


\section{6-Bromo-2,2'-bipyridine 17}<smiles>Brc1cccc(-c2ccccn2)n1</smiles>

Product 17 was synthesized via General procedure D using 2,6-dibromopyridine (106.6 mg, $0.45 \mathrm{mmol}$ ) and sulfonium salt 2a $(128.2 \mathrm{mg}, 0.3 \mathrm{mmol})$. Purification by $\mathrm{FCC}\left(40 \% \mathrm{Et}_{2} \mathrm{O}\right.$ in pentane) gave bipyridine 17 as a yellow solid (42.3 $\mathrm{mg}, 60 \%)$.

${ }^{1} \mathrm{H}$ NMR (500 MHz, Chloroform- $d$ ) $\delta 8.65$ (ddd, $\left.J=4.8,1.8,0.9 \mathrm{~Hz}, 1 \mathrm{H}\right), 8.46-8.30(\mathrm{~m}, 2 \mathrm{H}), 7.80$ $(\operatorname{td}, J=7.7,1.8 \mathrm{~Hz}, 1 \mathrm{H}), 7.65(\mathrm{t}, J=7.8 \mathrm{~Hz}, 1 \mathrm{H}), 7.48(\mathrm{dd}, J=7.8,0.9 \mathrm{~Hz}, 1 \mathrm{H}), 7.31(\mathrm{ddd}, J=7.5,4.8$, $1.2 \mathrm{~Hz}, 1 \mathrm{H})$.

${ }^{13} \mathrm{C}$ NMR (126 MHz, Chloroform-d) $\delta 157.5,154.6,149.3,141.7,139.3,137.1,128.1,124.4,121.6$, 119.8 .

Spectra are consistent with literature data. ${ }^{16}$

$N, N$-Dimethyl-5'-(trifluoromethyl)-[2,2'-bipyridin]-5-amine 18<smiles>CNc1ccc(-c2ccc(C(F)(F)F)cn2)nc1</smiles>

Product 18 was synthesized via General procedure D using 6-bromo- $N, N$-dimethylpyridin-3-amine ( $90.5 \mathrm{mg}, 0.45 \mathrm{mmol})$ and sulfonium salt $2 \mathbf{2}$ ( $148.6 \mathrm{mg}, 0.3 \mathrm{mmol})$. Purification by FCC (25\% Et $2 \mathrm{O}$ in pentane) gave bipyridine $\mathbf{1 8}$ as a yellow oil (32.7 $\mathrm{mg}, 41 \%)$.

TLC: $R_{\mathrm{f}}=0.10\left(25 \% \mathrm{Et}_{2} \mathrm{O}\right.$ in pentane $)$.

${ }^{1} \mathrm{H}$ NMR (500 MHz, Chloroform- $d$ ) $\delta 8.83(\mathrm{dd}, J=2.2,1.1 \mathrm{~Hz}, 1 \mathrm{H}), 8.39(\mathrm{~d}, J=8.4 \mathrm{~Hz}, 1 \mathrm{H}), 8.30$ (d, $J=8.8 \mathrm{~Hz}, 1 \mathrm{H}), 8.19(\mathrm{~d}, J=3.1 \mathrm{~Hz}, 1 \mathrm{H}), 7.94(\mathrm{dd}, J=8.5,2.4 \mathrm{~Hz}, 1 \mathrm{H}), 7.06(\mathrm{dd}, J=8.9,3.1 \mathrm{~Hz}, 1 \mathrm{H})$, $3.07\left(\mathrm{~s}, 6 \mathrm{H}, \mathrm{NMe}_{2}\right)$.

${ }^{13} \mathrm{C}$ NMR (126 MHz, Chloroform- $d$ ) $\delta 160.0$ (C), 146.8 (C), 146.0 (q, $\left.J=4.2 \mathrm{~Hz}, \mathrm{CH}\right), 142.8$ (C), 134.0 $(\mathrm{CH}), 133.8(\mathrm{q}, J=3.5 \mathrm{~Hz}, \mathrm{CH}), 124.5\left(\mathrm{q}, J=32.8 \mathrm{~Hz}, C \mathrm{CF}_{3}\right), 124.2\left(\mathrm{q}, J=271.9 \mathrm{~Hz}, \mathrm{CF}_{3}\right), 122.2$ $(\mathrm{CH}), 119.2(\mathrm{CH}), 118.6(\mathrm{CH}), 40.0\left(\mathrm{NMe}_{2}\right)$.

${ }^{19}$ F NMR (376 MHz, Chloroform- $d$ ) $\delta$-62.2.

HRMS (ESI-TOF) $m / z$ : $[\mathrm{M}+\mathrm{H}]^{+}$Calcd for $\mathrm{C}_{13} \mathrm{H}_{13} \mathrm{~F}_{3} \mathrm{~N}_{3}$ 268.1056; Found 268.1057. 


\section{1-(4-(Trifluoromethyl)pyridin-2-yl)isoquinoline 19}<smiles>FC(F)(F)c1ccnc(-c2nccc3ccccc23)c1</smiles>

Product 19 was synthesized via General procedure D using 1-iodoisoquinoline (114.8 mg, $0.45 \mathrm{mmol}$ ) and sulfonium salt $2 \mathbf{2 d}(148.6 \mathrm{mg}, 0.3 \mathrm{mmol})$. Purification by $\mathrm{FCC}\left(20 \% \mathrm{Et}_{2} \mathrm{O}\right.$ in pentane) gave bipyridine 19 as a yellow solid (46.4 mg, 56\%).

TLC: $R_{\mathrm{f}}=0.17\left(20 \% \mathrm{Et}_{2} \mathrm{O}\right.$ in pentane).

${ }^{1} \mathrm{H}$ NMR (400 MHz, Chloroform- $d$ ) $\delta 8.98(\mathrm{dt}, J=5.1,0.8 \mathrm{~Hz}, 1 \mathrm{H}), 8.70(\mathrm{dd}, J=8.6,1.1 \mathrm{~Hz}, 1 \mathrm{H}), 8.66$ $(\mathrm{d}, J=5.7 \mathrm{~Hz}, 1 \mathrm{H}), 8.35(\mathrm{dt}, J=1.7,0.8 \mathrm{~Hz}, 1 \mathrm{H}), 7.99-7.88(\mathrm{~m}, 1 \mathrm{H}), 7.77(\mathrm{dd}, J=5.6,1.0 \mathrm{~Hz}, 1 \mathrm{H})$, 7.73 (ddd, $J=8.2,6.9,1.2 \mathrm{~Hz}, 1 \mathrm{H}), 7.67-7.60$ (m, 2H).

${ }^{13} \mathrm{C}$ NMR (101 MHz, Chloroform- $d$ ) $\delta 160.0(\mathrm{C}), 155.9(\mathrm{C}), 149.6(\mathrm{CH}), 142.1(\mathrm{CH}), 139.2(\mathrm{q}, J=34.2$ $\left.\mathrm{Hz}, \mathrm{CCF}_{3}\right), 137.4(\mathrm{C}), 130.4(\mathrm{CH}), 128.2(\mathrm{CH}), 127.5(\mathrm{CH}), 127.3(\mathrm{CH}), 126.7(\mathrm{C}), 123.0(\mathrm{q}, J=273.2$ $\left.\mathrm{Hz}, \mathrm{CF}_{3}\right), 122.1(\mathrm{CH}), 121.3(\mathrm{q}, J=3.8 \mathrm{~Hz}, \mathrm{CH}), 118.8(\mathrm{q}, J=3.4 \mathrm{~Hz}, \mathrm{CH})$.

${ }^{19}$ F NMR (376 MHz, Chloroform- $d$ ) $\delta$-64.7.

HRMS (ESI-TOF) $m / z$ : [M+H] $]^{+}$Calcd for $\mathrm{C}_{15} \mathrm{H}_{10} \mathrm{~F}_{3} \mathrm{~N}_{2} 275.0791$; Found 275.0782.

\section{$N, N$-Dimethyl-4'-(trifluoromethyl)-[2,2'-bipyridin]-4-amine 20}<smiles>CN(C)c1ccnc(-c2cc(C(F)(F)F)ccn2)c1</smiles>

Product 20 was synthesized via General procedure D using 2-bromo- $N, N$-dimethylpyridin-4-amine ( $90.5 \mathrm{mg}, 0.45 \mathrm{mmol})$ and sulfonium salt $2 d$ (148.6 mg, $0.3 \mathrm{mmol})$. Purification by FCC ( $20 \% \mathrm{Et}_{2} \mathrm{O}$ in pentane) gave bipyridine $\mathbf{2 0}$ as a yellow solid (40.0 mg, 50\%).

TLC: $R_{\mathrm{f}}=0.15\left(25 \% \mathrm{Et}_{2} \mathrm{O}\right.$ in pentane $)$.

${ }^{1} \mathrm{H}$ NMR $(500 \mathrm{MHz}$, Chloroform- $d$ ) $\delta 8.81(\mathrm{~d}, J=5.0 \mathrm{~Hz}, 1 \mathrm{H}), 8.67(\mathrm{dt}, J=1.8,0.9 \mathrm{~Hz}, 1 \mathrm{H}), 8.33(\mathrm{~d}, J$ $=5.9 \mathrm{~Hz}, 1 \mathrm{H}), 7.73(\mathrm{~d}, J=2.7 \mathrm{~Hz}, 1 \mathrm{H}), 7.48(\mathrm{ddd}, J=5.1,1.8,0.8 \mathrm{~Hz}, 1 \mathrm{H}), 6.57(\mathrm{dd}, J=5.9,2.7 \mathrm{~Hz}$, 1H), $3.11\left(\mathrm{~s}, 6 \mathrm{H}, \mathrm{NMe}_{2}\right)$. 
${ }^{13} \mathrm{C}$ NMR (126 MHz, Chloroform- $d$ ) $\delta 158.6(\mathrm{C}), 155.4(\mathrm{C}), 154.9(\mathrm{C}), 149.8(\mathrm{CH}), 149.6(\mathrm{CH}), 139.3$ (q, $\left.J=33.9 \mathrm{~Hz}, C \mathrm{CF}_{3}\right), 123.2\left(\mathrm{q}, J=273.3 \mathrm{~Hz}, \mathrm{CF}_{3}\right), 118.9$ (d, $\left.J=3.5 \mathrm{~Hz}, \mathrm{CH}\right), 117.2(\mathrm{~d}, J=3.9 \mathrm{~Hz}$, $\mathrm{CH}), 107.4(\mathrm{CH}), 104.2(\mathrm{CH}), 39.5\left(\mathrm{NMe}_{2}\right)$.

${ }^{19} \mathrm{~F}$ NMR (282 MHz, Chloroform- $\left.d\right) \delta-64.7$.

HRMS (ESI-TOF) $m / z$ : [M+H] $]^{+}$Calcd for $\mathrm{C}_{13} \mathrm{H}_{13} \mathrm{~F}_{3} \mathrm{~N}_{3}$ 268.1056; Found 268.1058.

\section{5-Methoxy-6'-(trifluoromethyl)-2,2'-bipyridine 21}<smiles>COc1ccc(-c2cccc(C(F)(F)F)n2)nc1</smiles>

Product 21 was synthesized via General procedure D using 2-bromo-6-trifluoromethylpyridine (101.7 $\mathrm{mg}, 0.45 \mathrm{mmol})$ and sulfonium salt $\mathbf{2 g}(137.2 \mathrm{mg}, 0.3 \mathrm{mmol})$. Purification by FCC $\left(100 \% \mathrm{CH}_{2} \mathrm{Cl}_{2}\right)$ gave bipyridine 21 as a white solid (23.4 $\mathrm{mg}, 31 \%)$.

TLC: $R_{\mathrm{f}}=0.28\left(100 \% \mathrm{CH}_{2} \mathrm{Cl}_{2}\right)$.

${ }^{1} \mathrm{H}$ NMR $(500 \mathrm{MHz}$, Chloroform- $d$ ) $\delta 8.53(\mathrm{~d}, J=8.0 \mathrm{~Hz}, 1 \mathrm{H}), 8.47(\mathrm{~d}, J=8.7 \mathrm{~Hz}, 1 \mathrm{H}), 8.37(\mathrm{~d}, J=2.9$ $\mathrm{Hz}, 1 \mathrm{H}), 7.94$ (t, $J=7.9 \mathrm{~Hz}, 1 \mathrm{H}), 7.62$ (d, $J=7.7 \mathrm{~Hz}, 1 \mathrm{H}), 7.32$ (dd, $J=8.7,3.0 \mathrm{~Hz}, 1 \mathrm{H}), 3.93$ (s, 3H, $\mathrm{OMe})$.

${ }^{13} \mathrm{C}$ NMR (126 MHz, Chloroform- $d$ ) $\delta 156.8(\mathrm{C}), 156.6(\mathrm{C}), 147.7$ (q, $\left.J=34.6 \mathrm{~Hz}, C \mathrm{CF}_{3}\right), 147.6(\mathrm{C})$, $138.2(\mathrm{CH}), 137.2(\mathrm{CH}), 122.9(\mathrm{CH}), 122.5(\mathrm{CH}), 121.8\left(\mathrm{q}, J=274.7 \mathrm{~Hz}, \mathrm{CF}_{3}\right), 121.0(\mathrm{CH}), 119.4(\mathrm{q}$, $J=2.7 \mathrm{~Hz}, \mathrm{CH}), 55.8(\operatorname{app} \mathrm{d}, J=1.9 \mathrm{~Hz}, \mathrm{OMe})$.

${ }^{19} \mathrm{~F}$ NMR (282 MHz, Chloroform- $\left.d\right) \delta-68.1$.

HRMS (ESI-TOF) m/z: [M+H] Calcd for $\mathrm{C}_{12} \mathrm{H}_{10} \mathrm{~F}_{3} \mathrm{~N}_{2} \mathrm{O}$ 255.0740; Found 255.0741.

5-Methoxy-5'-methyl-2,2'-bipyridine 22<smiles>COc1ccc(-c2ccc(C)cn2)nc1</smiles>

Product 22 was synthesized via General procedure D using 2-fluoro-5-methylpyridine (77.4 mg, 0.45 mmol) and sulfonium salt $\mathbf{2 g}$ (137.2 $\mathrm{mg}, 0.3 \mathrm{mmol})$. Purification by FCC ( $5 \% \mathrm{MeOH}$ in $\mathrm{CH}_{2} \mathrm{Cl}_{2}$ ) gave bipyridine 22 as a yellow solid (37.6 $\mathrm{mg}, 63 \%)$.

TLC: $R_{\mathrm{f}}=0.07\left(5 \% \mathrm{MeOH}\right.$ in $\left.\mathrm{CH}_{2} \mathrm{Cl}_{2}\right)$. 
${ }^{1} \mathrm{H}$ NMR (400 MHz, Chloroform- $d$ ) $\delta 8.46-8.44(\mathrm{~m}, 1 \mathrm{H}), 8.34(\mathrm{~d}, J=2.9 \mathrm{~Hz}, 1 \mathrm{H}), 8.29$ (d, $J=8.7 \mathrm{~Hz}$, $1 \mathrm{H}), 8.18(\mathrm{~d}, J=8.1 \mathrm{~Hz}, 1 \mathrm{H}), 7.60-7.55(\mathrm{~m}, 1 \mathrm{H}), 7.29$ (dd, $J=8.8,3.0 \mathrm{~Hz}, 1 \mathrm{H}), 3.89$ (s, 3H, OMe), $2.36\left(\mathrm{~s}, 3 \mathrm{H}, \mathrm{CH}_{3}\right)$.

${ }^{13} \mathrm{C}$ NMR (101 MHz, Chloroform- $d$ ) $\delta$ 156.0, 153.7, 149.6, 149.3, 137.5, 136.9, 132.6, 121.5, 121.1, 120.0, $55.8(\mathrm{OMe}), 18.4\left(\mathrm{CH}_{3}\right)$.

Spectra were consistent with literature data. ${ }^{20}$

\section{3,3'-Dimethyl-2,2'-bipyridine 23}<smiles>Cc1cccnc1-c1ncccc1C</smiles>

Product 23 was synthesized via General procedure D using 2-bromo-3-methylpyridine (77.4 mg, 0.45 mmol) and sulfonium salt $\mathbf{2 b}(132.4 \mathrm{mg}, 0.3 \mathrm{mmol})$. Purification by FCC (40\% $\mathrm{Et}_{2} \mathrm{O}$ in pentane) gave bipyridine 23 as an orange oil (50.7 $\mathrm{mg}, 92 \%)$.

${ }^{1} \mathrm{H}$ NMR (400 MHz, Chloroform- $d$ ) $\delta 8.50(\mathrm{~d}, J=4.8 \mathrm{~Hz}, 2 \mathrm{H}), 7.60(\mathrm{~d}, J=7.6 \mathrm{~Hz}, 2 \mathrm{H}), 7.21$ (dd, $J=$ $7.8,4.7 \mathrm{~Hz}, 2 \mathrm{H}), 2.14(\mathrm{~s}, 6 \mathrm{H})$.

${ }^{13} \mathrm{C}$ NMR (101 MHz, Chloroform- $d$ ) $\delta$ 157.7, 146.7, 138.4, 131.7, 123.0, 18.6.

Spectra are consistent with literature data. ${ }^{21}$

1-(3-Methylpyridin-2-yl)isoquinoline 24<smiles>Cc1cccnc1-c1nccc2ccccc12</smiles>

Product 24 was synthesized via General procedure D using 1-iodoisoquinoline (114.8 mg, $0.45 \mathrm{mmol}$ ) and sulfonium salt $\mathbf{2 b}(132.4 \mathrm{mg}, 0.3 \mathrm{mmol})$. Purification by $\mathrm{FCC}\left(90 \% \mathrm{Et}_{2} \mathrm{O}\right.$ in pentane) gave bipyridine 24 as a yellow oil (57.1 $\mathrm{mg}, 86 \%)$.

TLC: $R_{\mathrm{f}}=0.20\left(90 \% \mathrm{Et}_{2} \mathrm{O}\right.$ in pentane).

${ }^{1} \mathrm{H}$ NMR (500 MHz, Chloroform- $d$ ) $\delta 8.62(\mathrm{~d}, J=5.7 \mathrm{~Hz}, 1 \mathrm{H}), 8.59(\mathrm{dd}, J=4.9,1.6 \mathrm{~Hz}, 1 \mathrm{H}), 7.94-$ $7.83(\mathrm{~m}, 1 \mathrm{H}), 7.68$ (app ddd, $J=11.2,7.7,5.9 \mathrm{~Hz}, 4 \mathrm{H}), 7.49$ (ddd, $J=7.5,6.9,1.2 \mathrm{~Hz}, 1 \mathrm{H}), 7.32$ (dd, $J$ $=7.8,4.8 \mathrm{~Hz}, 1 \mathrm{H}), 2.17(\mathrm{~s}, 3 \mathrm{H})$.

${ }^{13} \mathrm{C}$ NMR (126 MHz, Chloroform-d) $\delta 159.2,156.8,146.7,142.1,138.6,136.8,132.8,130.3,127.5$, $127.1,127.03,127.00,123.3,120.7,18.9$. 
Spectra were consistent with literature data. ${ }^{23}$

\section{1,1'-Biisoquinoline 25}<smiles>c1ccc2c(-c3nccc4ccccc34)nccc2c1</smiles>

Product 25 was synthesized via General procedure D using 1-iodoisoquinoline (114.8 mg, $0.45 \mathrm{mmol}$ ) and sulfonium salt $2 \mathrm{c}(143.3 \mathrm{mg}, 0.3 \mathrm{mmol})$. Purification by $\mathrm{FCC}\left(50 \% \mathrm{Et}_{2} \mathrm{O}\right.$ in pentane $)$ gave bipyridine 25 as a yellow oil ( $58.5 \mathrm{mg}, 76 \%$ ).

The reaction was scaled up and product 25 was synthesized via General procedure D using 1iodoisoquinoline (2.96 g, $11.6 \mathrm{mmol})$ and sulfonium salt $2 \mathrm{c}(3.68 \mathrm{~g}, 7.7 \mathrm{mmol})$. Purification by FCC (50\% $\mathrm{Et}_{2} \mathrm{O}$ in pentane) gave bipyridine 25 as a yellow oil ( $\left.0.91 \mathrm{~g}, 46 \%\right)$.

${ }^{1} \mathrm{H}$ NMR (400 MHz, Chloroform- $d$ ) $\delta 8.73$ (br s, 2H), $7.93(\mathrm{~d}, J=8.2 \mathrm{~Hz}, 2 \mathrm{H}), 7.81$ (d, $\left.J=5.5 \mathrm{~Hz}, 2 \mathrm{H}\right)$, $7.75(\mathrm{dd}, J=8.5,1.2 \mathrm{~Hz}, 2 \mathrm{H}), 7.69$ (ddd, $J=8.2,6.9,1.2 \mathrm{~Hz}, 2 \mathrm{H}), 7.47$ (ddd, $J=8.3,6.9,1.3 \mathrm{~Hz}, 2 \mathrm{H}$ ).

${ }^{13} \mathrm{C}$ NMR (101 MHz, Chloroform- $d$ ) $\delta 158.2$ (br), 142.0, 136.9, 130.4, 128.0, 127.7, 127.3, 127.0, 121.2 (br).

Spectra are consistent with literature data. ${ }^{24}$

1-(6-Bromopyridin-2-yl)isoquinoline 26<smiles>Brc1cccc(-c2nccc3ccccc23)n1</smiles>

Product 26 was synthesized via General procedure D using 2,6-dibromopyridine (106.6 mg, $0.45 \mathrm{mmol})$ and sulfonium salt $2 \mathrm{c}(143.3 \mathrm{mg}, 0.3 \mathrm{mmol})$. Purification by $\mathrm{FCC}\left(40 \% \mathrm{Et}_{2} \mathrm{O}\right.$ in pentane) gave bipyridine 26 as an off-yellow solid (44.1 $\mathrm{mg}, 52 \%)$.

TLC: $R_{\mathrm{f}}=0.29\left(25 \% \mathrm{Et}_{2} \mathrm{O}\right.$ in pentane).

${ }^{1} \mathrm{H}$ NMR (500 MHz, Chloroform- $d$ ) $\delta 8.66(\mathrm{dd} J=8.6,1.0 \mathrm{~Hz}, 1 \mathrm{H}), 8.61(\mathrm{~d}, J=5.6 \mathrm{~Hz}, 1 \mathrm{H}), 8.00$ (dd, $J=7.7,0.9 \mathrm{~Hz}, 1 \mathrm{H}), 7.88(\mathrm{dt}, J=8.3,1.0 \mathrm{~Hz}, 1 \mathrm{H}), 7.76(\mathrm{t}, J=7.8 \mathrm{~Hz}, 1 \mathrm{H}), 7.74-7.68(\mathrm{~m}, 2 \mathrm{H}), 7.64$ (ddd, $J=8.3,6.9,1.3 \mathrm{~Hz}, 1 \mathrm{H}), 7.60(\mathrm{dd}, J=7.9,0.9 \mathrm{~Hz}, 1 \mathrm{H})$. 
${ }^{13} \mathrm{C}$ NMR (126 MHz, Chloroform- $d$ ) $\delta 159.3(\mathrm{C}), 155.6(\mathrm{C}), 141.8(\mathrm{CH}), 140.6(\mathrm{C}), 139.2(\mathrm{CH}), 137.2$

(C), $130.2(\mathrm{CH}), 128.0(\mathrm{CH}), 127.7(\mathrm{CH}), 127.4(\mathrm{CH}), 127.0(\mathrm{CH}), 126.6(\mathrm{C}), 124.1(\mathrm{CH}), 121.8(\mathrm{CH})$.

HRMS (ESI-TOF) $m / z$ : [M+H] $]^{+}$Calcd for $\mathrm{C}_{14} \mathrm{H}_{10} \mathrm{BrN}_{2}$ 285.0022, 287.0001; Found 285.0023, 287.0003.

\section{2-(Isoquinolin-1-yl)- $N, N$-dimethylpyridin-4-amine 27}<smiles>CN(C)c1ccnc(-c2nccc3ccccc23)c1</smiles>

Product 27 was synthesized via General procedure D using 2-bromo- $N, N$-dimethylpyridin-4-amine (90.5 mg, $0.45 \mathrm{mmol}$ ) and sulfonium salt $2 \mathrm{c}$ ( $143.3 \mathrm{mg}, 0.3 \mathrm{mmol})$. Purification by FCC (100\% EtOAc) gave bipyridine 27 as a white solid $(56.6 \mathrm{mg}, 76 \%)$.

TLC: $R_{\mathrm{f}}=0.20(100 \%$ EtOAc $)$.

${ }^{1} \mathrm{H}$ NMR (500 MHz, Chloroform- $d$ ) $\delta 8.59(\mathrm{~d}, J=5.7 \mathrm{~Hz}, 1 \mathrm{H}), 8.54(\mathrm{dd}, J=8.6,1.1 \mathrm{~Hz}, 1 \mathrm{H}), 8.40(\mathrm{dd}$, $J=6.0,0.5 \mathrm{~Hz}, 1 \mathrm{H}), 7.85(\mathrm{dt}, J=8.2,1.0 \mathrm{~Hz}, 1 \mathrm{H}), 7.70-7.64(\mathrm{~m}, 2 \mathrm{H}), 7.56(\mathrm{ddd}, J=8.4,6.8,1.3 \mathrm{~Hz}$, $1 \mathrm{H}), 7.14(\mathrm{~d}, J=2.6 \mathrm{~Hz}, 1 \mathrm{H}), 6.61(\mathrm{dd}, J=6.0,2.7 \mathrm{~Hz}, 1 \mathrm{H}), 3.09$ (s, 6H, $\mathrm{NMe}_{2}$ ).

${ }^{13} \mathrm{C}$ NMR (126 MHz, Chloroform- $d$ ) $\delta 159.4,158.6,155.3,149.0,141.9,137.2,130.1,128.3,127.5$, $127.0,126.8,121.0,108.0,106.3,39.4\left(\mathrm{NMe}_{2}\right)$.

HRMS (ESI-TOF) $m / z$ : [M+H] $]^{+}$Calcd for $\mathrm{C}_{16} \mathrm{H}_{16} \mathrm{~N}_{3} 250.1339$; Found 250.1341 .

6,6'-Dibromo-2,2'-bipyridine 28<smiles>Brc1cccc(-c2cccc(Br)n2)n1</smiles>

Product 28 was synthesized via General procedure D using 2,6-dibromopyridine (106.6 mg, $0.45 \mathrm{mmol}$ ) and sulfonium salt $2 \mathbf{i}$ (151.9 mg, $0.3 \mathrm{mmol})$. Purification by FCC (5\% $\mathrm{Et}_{2} \mathrm{O}$ in pentane), followed by precipitation in $\mathrm{Et}_{2} \mathrm{O}$ gave bipyridine 28 as a white solid (12.4 $\left.\mathrm{mg}, 13 \%\right)$.

TLC: $R_{\mathrm{f}}=0.59\left(5 \% \mathrm{Et}_{2} \mathrm{O}\right.$ in pentane $)$.

${ }^{1} \mathrm{H}$ NMR (400 MHz, Chloroform- $d$ ) $\delta 8.38(\mathrm{dd}, J=7.8,0.7 \mathrm{~Hz}, 2 \mathrm{H}), 7.67(\mathrm{t}, J=7.8 \mathrm{~Hz}, 2 \mathrm{H}), 7.51$ (dd, $J=7.8,0.7 \mathrm{~Hz}, 2 \mathrm{H})$.

${ }^{13} \mathrm{C}$ NMR (101 MHz, Chloroform-d) $\delta 155.8,141.7,139.5,128.7,120.3$. 
Spectra were consistent with literature data. ${ }^{25}$

6'-Bromo-2,3'-bipyridine 29<smiles>Brc1ccc(-c2ccccn2)cn1</smiles>

Product 29 was synthesized via a modification to General procedure D. 2,5-Dibromopyridine (106.6 $\mathrm{mg}, 0.45 \mathrm{mmol})$ was dissolved in dry THF $(0.75 \mathrm{~mL})$ and added dropwise to a solution of $n$-BuLi $(0.19$ $\mathrm{mL}, 0.45 \mathrm{mmol}$, 1.5 equiv., $2.4 \mathrm{M}$ in hexanes $)$ in dry THF $(0.75 \mathrm{~mL})$ over $2 \mathrm{~min}$. The solution of sulfonium salt 2a (128.2 $\mathrm{mg}, 0.3 \mathrm{mmol})$ in dry THF $(1.5 \mathrm{~mL})$ was then added dropwise down the side of the vial to the lithiated pyridine solution over $2 \mathrm{~min}$. Purification by $\mathrm{FCC}\left(10 \% \mathrm{Et}_{2} \mathrm{O}\right.$ in $\left.\mathrm{CH}_{2} \mathrm{Cl}_{2}\right)$ gave bipyridine 29 as a white solid (45.0 $\mathrm{mg}, 64 \%)$.

TLC: $R_{\mathrm{f}}=0.11\left(20 \% \mathrm{Et}_{2} \mathrm{O}\right.$ in $\left.\mathrm{CH}_{2} \mathrm{Cl}_{2}\right)$.

${ }^{1} \mathrm{H}$ NMR (500 MHz, Chloroform- $d$ ) $\delta 8.94(\mathrm{~d}, J=2.5 \mathrm{~Hz}, 1 \mathrm{H}), 8.73-8.69(\mathrm{~m}, 1 \mathrm{H}), 8.20(\mathrm{dd}, J=8.3$, $2.6 \mathrm{~Hz}, 1 \mathrm{H}), 7.80(\mathrm{td}, J=7.6,1.8 \mathrm{~Hz}, 1 \mathrm{H}), 7.75-7.71(\mathrm{~m}, 1 \mathrm{H}), 7.59(\mathrm{~d}, J=8.4 \mathrm{~Hz}, 1 \mathrm{H}), 7.31(\mathrm{ddd}, J$ $=7.3,4.9,0.9 \mathrm{~Hz}, 2 \mathrm{H})$.

${ }^{13} \mathrm{C}$ NMR (126 MHz, Chloroform-d) $\delta 153.7(\mathrm{C}), 150.3(\mathrm{CH}), 148.6(\mathrm{CH}), 142.7(\mathrm{C}), 137.3(\mathrm{CH}), 137.0$ $(\mathrm{CH}), 134.3(\mathrm{C}), 128.3(\mathrm{CH}), 123.3(\mathrm{CH}), 120.6(\mathrm{CH})$.

HRMS (ESI-TOF) $m / z$ : $[\mathrm{M}+\mathrm{H}]^{+}$Calcd for $\mathrm{C}_{10} \mathrm{H}_{8} \mathrm{BrN}_{2} 234.9865$, 236.9845; Found 234.9869, 236.9834.

\section{6'-Fluoro-2,3'-bipyridine 30}

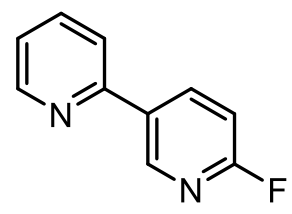

Product 30 was synthesized via General procedure D using 2-fluoro-5-iodopyridine (100.3 mg, 0.45 mmol) and sulfonium salt 2a (128.2 mg, $0.3 \mathrm{mmol})$. Purification by $\mathrm{FCC}\left(10 \% \mathrm{Et}_{2} \mathrm{O}\right.$ in $\left.\mathrm{CH}_{2} \mathrm{Cl}_{2}\right)$ gave bipyridine 30 as a white solid (36.1 $\mathrm{mg}, 69 \%)$.

TLC: $R_{\mathrm{f}}=0.43\left(10 \% \mathrm{Et}_{2} \mathrm{O}\right.$ in $\left.\mathrm{CH}_{2} \mathrm{Cl}_{2}\right)$.

${ }^{1} \mathrm{H}$ NMR (500 MHz, Chloroform- $d$ ) $\delta 8.78(\mathrm{~d}, J=2.5 \mathrm{~Hz}, 1 \mathrm{H}), 8.70(\mathrm{dd}, J=4.7,1.6 \mathrm{~Hz}, 1 \mathrm{H}), 8.44$ (td, $J=8.0,2.5 \mathrm{~Hz}, 1 \mathrm{H}), 7.78(\mathrm{td}, J=7.8,1.8 \mathrm{~Hz}, 1 \mathrm{H}), 7.70(\mathrm{~d}, J=8.1 \mathrm{~Hz}, 1 \mathrm{H}), 7.28(\mathrm{dd}, J=7.6,4.7 \mathrm{~Hz}$, $1 \mathrm{H}), 7.03(\mathrm{dd}, J=8.5,3.0 \mathrm{~Hz}, 1 \mathrm{H})$. 
${ }^{13} \mathrm{C}$ NMR (126 MHz, Chloroform- $d$ ) $\delta 164.1$ (d, $\left.J=240.4 \mathrm{~Hz}, \mathrm{CF}\right), 153.9(\mathrm{C}), 150.2(\mathrm{CH}), 146.3$ (d, $J$ $=15.3 \mathrm{~Hz}, \mathrm{CH}), 140.0(\mathrm{~d}, J=8.5 \mathrm{~Hz}, \mathrm{CH}), 137.2(\mathrm{CH}), 133.3$ (d, $J=4.5 \mathrm{~Hz}, \mathrm{C}), 123.0(\mathrm{CH}), 120.4$ (CH), 109.7 (d, $J=37.2 \mathrm{~Hz}, \mathrm{CH})$.

${ }^{19}$ F NMR (282 MHz, Chloroform- $\left.d\right) \delta-68.3(\mathrm{~d}, J=5.7 \mathrm{~Hz})$.

HRMS (ESI-TOF) $m / z$ : [M+H] $]^{+}$Calcd for $\mathrm{C}_{10} \mathrm{H}_{8} \mathrm{FN}_{2}$ 175.0666; Found 175.0667.

\section{6'-Methoxy-2,3'-bipyridine 31}

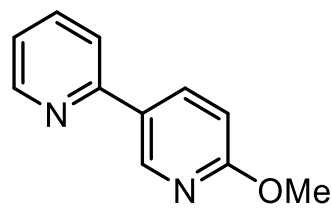

Product 31 was synthesized via General procedure D using 5-iodo-2-methoxypyridine (105.8 mg, 0.45 mmol) and sulfonium salt 2a (128.2 mg, $0.3 \mathrm{mmol})$. Purification by FCC (20\% EtOAc in pentane) gave bipyridine $\mathbf{3 1}$ as a yellow oil (32.5 $\mathrm{mg}, 58 \%)$.

TLC: $R_{\mathrm{f}}=0.25$ (20\% EtOAc in pentane).

${ }^{1} \mathrm{H}$ NMR (400 MHz, Chloroform- $d$ ) $\delta 8.73(\mathrm{~d}, J=2.4 \mathrm{~Hz}, 1 \mathrm{H}), 8.65(\mathrm{dt}, J=5.1,1.5 \mathrm{~Hz}, 1 \mathrm{H}), 8.23(\mathrm{dd}$, $J=8.6,2.7 \mathrm{~Hz}, 1 \mathrm{H}), 7.72(\mathrm{td}, J=7.7,1.8 \mathrm{~Hz}, 1 \mathrm{H}), 7.65(\mathrm{dt}, J=8.2,1.1 \mathrm{~Hz}, 1 \mathrm{H}), 7.20$ (ddd, $J=7.3$, $4.8,1.2 \mathrm{~Hz}, 1 \mathrm{H}), 6.83(\mathrm{~d}, J=8.6 \mathrm{~Hz}, 1 \mathrm{H}), 3.98(\mathrm{~s}, 3 \mathrm{H})$.

${ }^{13} \mathrm{C}$ NMR (101 MHz, Chloroform- $d$ ) $\delta$ 164.8, 155.2, 149.9, 145.6, 137.5, 137.0, 128.7, 122.1, 119.9, $111.0,53.8$

Spectra were consistent with literature data. ${ }^{17}$

\section{6'-(Trifluoromethyl)-2,3'-bipyridine 32}

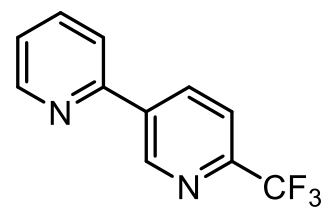

Product 32 was synthesized via General procedure D using 5-bromo-2-(trifluoromethyl)pyridine (101.7 $\mathrm{mg}, 0.45 \mathrm{mmol})$ and sulfonium salt $2 \mathrm{a}(128.2 \mathrm{mg}, 0.3 \mathrm{mmol})$. Purification by $\mathrm{FCC}\left(20 \% \mathrm{Et}_{2} \mathrm{O}\right.$ in pentane) gave bipyridine 32 as a yellow oil (35.8 $\mathrm{mg}, 53 \%)$.

TLC: $R_{\mathrm{f}}=0.18\left(25 \% \mathrm{Et}_{2} \mathrm{O}\right.$ in pentane $)$.

${ }^{1} \mathrm{H}$ NMR $(500 \mathrm{MHz}$, Chloroform- $d$ ) $\delta 9.30(\mathrm{~d}, J=2.1 \mathrm{~Hz}, 1 \mathrm{H}), 8.77$ (ddd, $J=4.8,1.8,1.0 \mathrm{~Hz}, 1 \mathrm{H}), 8.52$ (ddd, $J=8.2,2.2,0.8 \mathrm{~Hz}, 1 \mathrm{H}), 8.08-7.72(\mathrm{~m}, 3 \mathrm{H}), 7.36(\mathrm{ddd}, J=7.3,4.8,1.4 \mathrm{~Hz}, 1 \mathrm{H})$. 
${ }^{13} \mathrm{C}$ NMR (101 MHz, Chloroform- $d$ ) $\delta 153.4,150.5,148.5,148.2$ (q, $\left.J=34.7 \mathrm{~Hz}\right), 137.6,137.4,135.8$, $123.8,121.8(\mathrm{q}, J=274.0 \mathrm{~Hz}), 121.1,120.6(\mathrm{q}, J=2.7 \mathrm{~Hz})$.

${ }^{19}$ F NMR (376 MHz, Chloroform- $d$ ) $\delta$-67.9.

Spectra were consistent with literature data.$^{18}$

\section{6'-Methyl-2,3'-bipyridine 33}

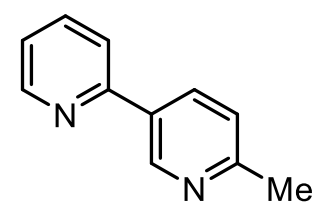

Product 33 was synthesized via General procedure D using 5-bromo-2-methylpyridine (77.4 mg, 0.45 $\mathrm{mmol})$ and sulfonium salt $\mathbf{2 a}(128.2 \mathrm{mg}, 0.3 \mathrm{mmol})$. Purification by $\mathrm{FCC}\left(80 \% \mathrm{Et}_{2} \mathrm{O}\right.$ in pentane) gave bipyridine 33 as a yellow oil (44.8 $\mathrm{mg}, 88 \%)$.

${ }^{1} \mathrm{H}$ NMR (400 MHz, Chloroform- $d$ ) $\delta 9.07$ (d, $J=2.4 \mathrm{~Hz}, 1 \mathrm{H}$ ), 8.70 (ddd, $J=4.8,2.5,1.2 \mathrm{~Hz}, 1 \mathrm{H}$ ), 8.22 $(\mathrm{dt}, J=8.1,2.6 \mathrm{~Hz}, 1 \mathrm{H}), 7.86-7.64(\mathrm{~m}, 2 \mathrm{H}), 7.25(\mathrm{ddt}, J=7.2,3.6,1.8 \mathrm{~Hz}, 2 \mathrm{H}), 2.61(\mathrm{~s}, 3 \mathrm{H})$.

${ }^{13} \mathrm{C}$ NMR (101 MHz, Chloroform- $d$ ) $\delta 158.9,155.0,150.0,147.6,137.0,134.7,132.1,123.3,122.6$, 120.3, 24.3.

Spectra are consistent with literature data. ${ }^{19}$

5-(Trifluoromethyl)-2,3'-bipyridine 34<smiles>FC(F)(F)c1ccc(-c2cccnc2)nc1</smiles>

Product 34 was synthesized via General procedure D using 3-iodopyridine ( $92.3 \mathrm{mg}, 0.45 \mathrm{mmol})$ and sulfonium salt $2 \mathbf{f}$ ( $148.6 \mathrm{mg}, 0.3 \mathrm{mmol}$ ). Purification by $\mathrm{FCC}$ ( $45 \% \mathrm{Et}_{2} \mathrm{O}$ in pentane) gave bipyridine 34 as a yellow oil (58.0 $\mathrm{mg}, 86 \%)$.

TLC: $R_{\mathrm{f}}=0.13\left(25 \% \mathrm{Et}_{2} \mathrm{O}\right.$ in pentane $)$.

${ }^{1} \mathrm{H}$ NMR $(500 \mathrm{MHz}$, Chloroform- $d$ ) $\delta 9.24(\mathrm{~d}, J=2.3 \mathrm{~Hz}, 1 \mathrm{H}), 8.97(\mathrm{~d}, J=2.4 \mathrm{~Hz}, 1 \mathrm{H}), 8.71(\mathrm{~d}, J=4.6$ $\mathrm{Hz}, 1 \mathrm{H}), 8.36(\mathrm{dt}, J=8.1,1.9 \mathrm{~Hz}, 1 \mathrm{H}), 8.02(\mathrm{dd}, J=8.3,2.4 \mathrm{~Hz}, 1 \mathrm{H}), 7.88$ (d, $J=8.3 \mathrm{~Hz}, 1 \mathrm{H}), 7.44$ (dd, $J=8.0,4.8 \mathrm{~Hz}, 1 \mathrm{H})$. 
${ }^{13} \mathrm{C}$ NMR (126 MHz, Chloroform- $d$ ) $\delta 158.2(\mathrm{q}, J=1.4 \mathrm{~Hz}, \mathrm{C}), 151.1(\mathrm{CH}), 148.6(\mathrm{CH}), 147.1$ (q, $J=$ $4.2 \mathrm{~Hz}, \mathrm{CH}), 134.8(\mathrm{CH}), 134.4$ (q, $J=3.5 \mathrm{~Hz}, \mathrm{CH}), 133.6(\mathrm{C}), 125.7$ (q, $\left.J=33.3 \mathrm{~Hz}, C C F_{3}\right), 123.9$ $(\mathrm{CH}), 123.7$ (q, $\left.J=272.3 \mathrm{~Hz}, \mathrm{CF}_{3}\right), 120.1(\mathrm{CH})$.

${ }^{19} \mathrm{~F}$ NMR (470 MHz, Chloroform- $\left.d\right) \delta-62.4$.

HRMS (ESI-TOF) $m / z$ : $[\mathrm{M}+\mathrm{H}]^{+}$Calcd for $\mathrm{C}_{11} \mathrm{H}_{8} \mathrm{~F}_{3} \mathrm{~N}_{2} 225.0634$; Found 225.0634.

6'-Fluoro-5-(trifluoromethyl)-2,3'-bipyridine 35

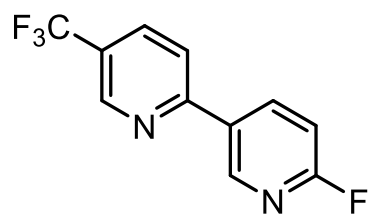

Product 35 was synthesized via General procedure D using 2-fluoro-5-iodopyridine (127.8 mg, 0.45 mmol) and sulfonium salt $2 \mathbf{f}(148.6 \mathrm{mg}, 0.3 \mathrm{mmol})$. Purification by $\mathrm{FCC}\left(20 \% \mathrm{Et}_{2} \mathrm{O}\right.$ in pentane) gave bipyridine 35 as a yellow solid (54.8 $\mathrm{mg}, 75 \%$ ).

TLC: $R_{\mathrm{f}}=0.29\left(25 \% \mathrm{Et}_{2} \mathrm{O}\right.$ in pentane $)$.

${ }^{1} \mathrm{H}$ NMR (500 MHz, Chloroform- $d$ ) $\delta 8.95(\mathrm{dt}, J=2.1,1.0 \mathrm{~Hz}, 1 \mathrm{H}), 8.87-8.84(\mathrm{~m}, 1 \mathrm{H}), 8.50$ (ddd, $J$ $=8.6,7.6,2.6 \mathrm{~Hz}, 1 \mathrm{H}), 8.05-7.96(\mathrm{~m}, 1 \mathrm{H}), 7.84(\mathrm{dt}, J=8.3,0.8 \mathrm{~Hz}, 1 \mathrm{H}), 7.07$ (ddd, $J=8.6,3.0,0.7$ $\mathrm{Hz}, 1 \mathrm{H})$.

${ }^{13} \mathrm{C}$ NMR (126 MHz, Chloroform- $d$ ) $\delta 164.7$ (d, $\left.J=242.7 \mathrm{~Hz}, \mathrm{CF}\right), 157.1(\mathrm{~d}, J=1.5 \mathrm{~Hz}), 147.1$ (q, $J=$ $4.0 \mathrm{~Hz}), 146.9$ (d, $J=15.7 \mathrm{~Hz}), 140.3$ (d, $J=8.5 \mathrm{~Hz}), 134.5$ (q, $J=3.6 \mathrm{~Hz}), 131.9$ (d, $J=4.8 \mathrm{~Hz}), 125.8$ (q, $\left.J=33.3 \mathrm{~Hz}, C \mathrm{CF}_{3}\right), 123.6\left(\mathrm{q}, J=272.3 \mathrm{~Hz}, \mathrm{CF}_{3}\right), 119.8,110.0(\mathrm{~d}, J=37.6 \mathrm{~Hz})$.

${ }^{19}$ F NMR (470 MHz, Chloroform- $\left.d\right) \delta-62.4,-66.5(\mathrm{~d}, J=4.6 \mathrm{~Hz})$.

HRMS (ESI-TOF) $m / z$ : $[\mathrm{M}+\mathrm{H}]^{+}$Calcd for $\mathrm{C}_{11} \mathrm{H}_{7} \mathrm{~F}_{4} \mathrm{~N}_{2} 243.0540$; Found 243.0539.

\section{6'-Fluoro- $N, N$-dimethyl-[2,3'-bipyridin]-5-amine 36}

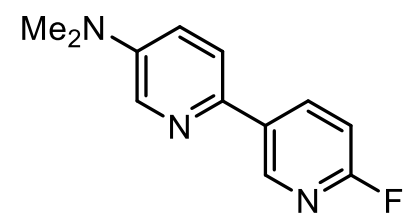

Product 36 was synthesized via General procedure D using 2-bromo- $N, N$-dimethylpyridin-4-amine ( $90.5 \mathrm{mg}, 0.45 \mathrm{mmol})$ and sulfonium salt $\mathbf{2 h}(148.6 \mathrm{mg}, 0.3 \mathrm{mmol})$. Purification by $\mathrm{FCC}\left(40 \% \mathrm{Et}_{2} \mathrm{O}\right.$ in pentane) gave bipyridine 36 as a yellow oil (26.7 $\mathrm{mg}, 41 \%)$.

TLC: $R_{\mathrm{f}}=0.10\left(40 \% \mathrm{Et}_{2} \mathrm{O}\right.$ in pentane). 
${ }^{1} \mathrm{H}$ NMR (500 MHz, Chloroform- $d$ ) $\delta 8.70-8.67(\mathrm{~m}, 1 \mathrm{H}), 8.34$ (ddd, $\left.J=8.6,7.8,2.6 \mathrm{~Hz}, 1 \mathrm{H}\right), 8.21$ (d, $J=3.1 \mathrm{~Hz}, 1 \mathrm{H}), 7.60-7.49$ (m, 1H), 7.04 (dd, $J=8.8,3.1 \mathrm{~Hz}, 1 \mathrm{H}), 7.00-6.93$ (m, 1H), 3.04 (s, 6H, $\mathrm{NMe}_{2}$ ).

${ }^{13} \mathrm{C}$ NMR (126 MHz, Chloroform- $d$ ) $\delta 163.2(\mathrm{~d}, J=238.5 \mathrm{~Hz}, \mathrm{CF}), 145.6(\mathrm{C}), 144.9$ (d, $J=14.9 \mathrm{~Hz}$, CH), 141.6 (C), 138.7 (d, $J=7.9$ Hz, CH), 134.9 (CH), 133.6 (d, $J=4.4 \mathrm{~Hz}, \mathrm{C}), 120.3$ (CH), 119.2 (CH), $109.4(\mathrm{~d}, J=37.4 \mathrm{~Hz}, \mathrm{CH}), 40.2\left(\mathrm{NMe}_{2}\right)$.

${ }^{19} \mathrm{~F}$ NMR $(470 \mathrm{MHz}$, Chloroform- $d) \delta-70.9(\mathrm{~d}, J=4.8 \mathrm{~Hz})$.

HRMS (ESI-TOF) $m / z$ : [M+H] $]^{+}$Calcd for $\mathrm{C}_{12} \mathrm{H}_{13} \mathrm{FN}_{3} 218.1088$; Found 218.1090.

\section{6'-Fluoro-5-methoxy-2,3'-bipyridine 37}

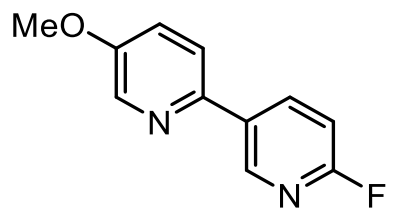

Product 37 was synthesized via General procedure D using 2-fluoro-5-iodopyridine (100.3 mg, 0.45 $\mathrm{mmol}$ ) and sulfonium salt $\mathbf{2 g}(137.2 \mathrm{mg}, 0.3 \mathrm{mmol})$. Purification by $\mathrm{FCC}\left(50 \% \mathrm{Et}_{2} \mathrm{O}\right.$ in pentane) gave bipyridine 37 as a white solid (49.9 $\mathrm{mg}, 82 \%)$.

TLC: $R_{\mathrm{f}}=0.29\left(50 \% \mathrm{Et}_{2} \mathrm{O}\right.$ in pentane $)$.

${ }^{1} \mathrm{H}$ NMR (400 MHz, Chloroform- $d$ ) $\delta 8.68(\mathrm{~d}, J=2.5 \mathrm{~Hz}, 1 \mathrm{H}), 8.35(\mathrm{~d}, J=2.7 \mathrm{~Hz}, 1 \mathrm{H}), 8.32(\mathrm{dd}, J=$ $8.1,2.6 \mathrm{~Hz}, 1 \mathrm{H}), 7.61(\mathrm{~d}, J=8.6 \mathrm{~Hz}, 1 \mathrm{H}), 7.25(\mathrm{dd}, J=8.7,3.0 \mathrm{~Hz}, 1 \mathrm{H}), 6.96(\mathrm{dd}, J=8.6,3.0 \mathrm{~Hz}, 1 \mathrm{H})$, $3.87(\mathrm{~s}, 3 \mathrm{H}, \mathrm{OMe})$.

${ }^{13} \mathrm{C}$ NMR (101 MHz, Chloroform- $d$ ) $\delta 163.6$ (d, $\left.J=239.6 \mathrm{~Hz}, \mathrm{CF}\right), 155.5(\mathrm{C}), 146.3(\mathrm{C}), 145.5$ (d, $J=$ 15.2 Hz, CH), 139.4 (d, J=8.0 Hz, CH), $137.9(\mathrm{CH}), 133.0$ (d, J=4.7 Hz, C), 121.3 (CH), 120.7 (CH), $109.5(\mathrm{~d}, J=37.4 \mathrm{~Hz}, \mathrm{CH}), 55.8(\mathrm{OMe})$.

${ }^{19} \mathrm{~F}$ NMR (282 MHz, Chloroform- $\left.d\right) \delta-69.5(\mathrm{~d}, J=5.8 \mathrm{~Hz})$.

HRMS (ESI-TOF) $m / z$ : [M+H] $]^{+}$Calcd for $\mathrm{C}_{11} \mathrm{H}_{10} \mathrm{FN}_{2} \mathrm{O}$ 205.0772; Found 205.0774. 


\section{3-Methyl-2,3'-bipyridine 38}<smiles>Cc1cccnc1-c1cccnc1</smiles>

Product 38 was synthesized via General procedure D using 3-iodopyridine $(92.3 \mathrm{mg}, 0.45 \mathrm{mmol})$ and sulfonium salt $\mathbf{2 b}$ (132.4 mg, $0.3 \mathrm{mmol})$. Purification by FCC ( $90 \% \mathrm{Et}_{2} \mathrm{O}$ in pentane) gave bipyridine 38 as a yellow oil (26.7 $\mathrm{mg}, 52 \%)$.

${ }^{1} \mathrm{H}$ NMR (400 MHz, Chloroform- $d$ ) $\delta 8.81-8.75(\mathrm{~m}, 1 \mathrm{H}), 8.62$ (dd, $\left.J=4.9,1.7 \mathrm{~Hz}, 1 \mathrm{H}\right), 8.54$ (ddd, $J$ $=4.7,1.7,0.7 \mathrm{~Hz}, 1 \mathrm{H}), 7.87(\mathrm{ddd}, J=7.8,2.2,1.6 \mathrm{~Hz}, 1 \mathrm{H}), 7.60(\mathrm{ddd} J=7.7,1.5,0.7 \mathrm{~Hz}, 1 \mathrm{H}), 7.38$ (ddd, $J=7.9,4.8,0.9 \mathrm{~Hz}, 1 \mathrm{H}), 7.22(\mathrm{dd}, J=7.7,4.7 \mathrm{~Hz}, 1 \mathrm{H}), 2.36(\mathrm{~s}, 3 \mathrm{H})$.

${ }^{13} \mathrm{C}$ NMR (101 MHz, Chloroform- $d$ ) $\delta 155.6,150.0,149.1,147.5,138.9,136.6,136.4,131.4,123.3$, $122.9,20.0$.

Spectra were consistent with literature data. ${ }^{22}$

\section{6-Bromo-6'-methoxy-2,3'-bipyridine 39}<smiles>COc1ccc(-c2cccc(Br)n2)cn1</smiles>

Product 39 was synthesized via General procedure D using 2-methoxy-5-iodopyridine (105.7 mg, 0.45 mmol) and sulfonium salt $2 \mathbf{i}(151.9 \mathrm{mg}, 0.3 \mathrm{mmol})$. Purification by FCC ( $30 \% \mathrm{Et}_{2} \mathrm{O}$ in pentane) gave bipyridine 39 as a white solid (26.2 $\mathrm{mg}, 33 \%)$.

TLC: $R_{\mathrm{f}}=0.51\left(30 \% \mathrm{Et}_{2} \mathrm{O}\right.$ in pentane).

${ }^{1} \mathrm{H}$ NMR (400 MHz, Chloroform- $d$ ) $\delta 8.73$ (d, $\left.J=2.7 \mathrm{~Hz}, 1 \mathrm{H}\right), 8.26(\mathrm{dd}, J=8.6,2.7 \mathrm{~Hz}, 1 \mathrm{H}), 7.64-$ $7.55(\mathrm{~m}, 2 \mathrm{H}), 7.40(\mathrm{dd}, J=7.4,1.2 \mathrm{~Hz}, 1 \mathrm{H}), 6.83(\mathrm{~d}, J=8.7 \mathrm{~Hz}, 1 \mathrm{H}), 4.00$ (s, 3H, OMe).

${ }^{13} \mathrm{C}$ NMR (101 MHz, Chloroform- $d$ ) $\delta 165.2(\mathrm{C}), 156.3(\mathrm{C}), 145.8(\mathrm{CH}), 142.5(\mathrm{C}), 139.2(\mathrm{CH}), 137.6$ $(\mathrm{CH}), 127.2(\mathrm{C}), 126.3(\mathrm{CH}), 118.3(\mathrm{CH}), 111.2(\mathrm{CH}), 53.9(\mathrm{OMe})$.

HRMS (ESI-TOF) $m / z$ : $[\mathrm{M}+\mathrm{H}]^{+}$Calcd for $\mathrm{C}_{11} \mathrm{H}_{10} \mathrm{BrN}_{2} \mathrm{O}$ 264.9971, 266.9951; Found 264.9972, 266.9949. 
<smiles>Fc1ccc(-c2cccc(Br)n2)cn1</smiles>

Product 40 was synthesized via General procedure D using 2-fluoro-5-iodopyridine (100.3 mg, 0.45 mmol) and sulfonium salt $2 \mathbf{i}$ (151.9 mg, $0.3 \mathrm{mmol})$. Purification by $\mathrm{FCC}\left(30 \% \mathrm{Et}_{2} \mathrm{O}\right.$ in pentane) gave bipyridine $\mathbf{4 0}$ as a white solid (32.6 $\mathrm{mg}, 43 \%)$.

TLC: $R_{\mathrm{f}}=0.25\left(30 \% \mathrm{Et}_{2} \mathrm{O}\right.$ in pentane).

${ }^{1} \mathrm{H}$ NMR (500 MHz, Chloroform- $d$ ) $\delta 8.77$ (dt, $\left.J=2.7,0.8 \mathrm{~Hz}, 1 \mathrm{H}\right), 8.47$ (ddd, $\left.J=8.6,7.6,2.6 \mathrm{~Hz}, 1 \mathrm{H}\right)$, $7.71-7.59$ (m, 2H), $7.48(\mathrm{dd}, J=7.6,1.1 \mathrm{~Hz}, 1 \mathrm{H}), 7.04(\mathrm{ddd}, J=8.6,3.1,0.7 \mathrm{~Hz}, 1 \mathrm{H})$.

${ }^{13} \mathrm{C}$ NMR (101 MHz, Chloroform- $d$ ) $\delta 164.4$ (d, $\left.J=242.5 \mathrm{~Hz}, \mathrm{CF}\right), 154.9$ (C), 146.4 (d, $J=15.9 \mathrm{~Hz}$, CH), 142.7 (C), 140.2 (d, $J=8.6 \mathrm{~Hz}, \mathrm{CH}), 139.4(\mathrm{CH}), 131.7$ (d, $J=4.6 \mathrm{~Hz}, \mathrm{C}), 127.4(\mathrm{CH}), 118.9$ $(\mathrm{CH}), 109.9(\mathrm{~d}, J=37.5 \mathrm{~Hz}, \mathrm{CH})$.

${ }^{19}$ F NMR (282 MHz, Chloroform- $\left.d\right) \delta-67.1(\mathrm{~d}, J=5.7 \mathrm{~Hz})$.

HRMS (ESI-TOF) $m / z$ : $[\mathrm{M}+\mathrm{H}]^{+}$Calcd for $\mathrm{C}_{10} \mathrm{H}_{7} \mathrm{BrFN}_{2}$ 252.9771, 254.9751; Found 252.9775, 254.9738 .

1-(4'-(Trifluoromethyl)-[2,2'-bipyridin]-6-yl)isoquinoline 43<smiles>FC(F)(F)c1ccnc(-c2cccc(-c3nccc4ccccc34)n2)c1</smiles>

Product 43 was synthesized via General procedure D using $31(127.8 \mathrm{mg}, 0.45 \mathrm{mmol})$ and sulfonium salt $2 d$ (148.6 mg, $0.3 \mathrm{mmol})$. Purification by $\mathrm{FCC}\left(50 \% \mathrm{Et}_{2} \mathrm{O}\right.$ in pentane) gave terpyridine 43 as an off-green solid (21.0 mg, 20\%).

TLC: $R_{\mathrm{f}}=0.18(25 \%$ EtOAc in pentane).

${ }^{1} \mathrm{H}$ NMR (500 MHz, Chloroform- $d$ ) $\delta 8.89(\mathrm{~d}, J=5.0 \mathrm{~Hz}, 1 \mathrm{H}), 8.79(\mathrm{dd}, J=8.5,1.0 \mathrm{~Hz}, 1 \mathrm{H}), 8.75$ (dt, $J=1.6,0.8 \mathrm{~Hz}, 1 \mathrm{H}), 8.67$ (d, $J=5.6 \mathrm{~Hz}, 1 \mathrm{H}), 8.59$ (dd, $J=7.7,1.3 \mathrm{~Hz}, 1 \mathrm{H}), 8.13(\mathrm{dd}, J=7.8,1.3 \mathrm{~Hz}$, $1 \mathrm{H}), 8.08(\mathrm{t}, J=7.7 \mathrm{~Hz}, 1 \mathrm{H}), 7.98-7.91(\mathrm{~m}, 1 \mathrm{H}), 7.79-7.71(\mathrm{~m}, 2 \mathrm{H}), 7.62$ (ddd, $J=8.4,6.8,1.3 \mathrm{~Hz}$, 1H), $7.57-7.53(\mathrm{~m}, 1 \mathrm{H})$.

${ }^{13} \mathrm{C}$ NMR (126 MHz, Chloroform- $d$ ) $\delta 158.2$ (C), 157.7 (C), 157.3 (C), 153.6 (C), $150.3(\mathrm{CH}), 142.1$ $(\mathrm{CH}), 139.5\left(\mathrm{q}, J=34.0 \mathrm{~Hz}, C C F_{3}\right), 138.4(\mathrm{CH}), 137.4(\mathrm{C}), 130.3(\mathrm{CH}), 128.0(\mathrm{CH}), 127.7(\mathrm{CH}), 127.2$ 
$(\mathrm{CH}), 127.0(\mathrm{CH}), 126.2(\mathrm{C}), 123.1\left(\mathrm{q}, J=273.2 \mathrm{~Hz}, \mathrm{CF}_{3}\right), 121.7(\mathrm{CH}), 120.8(\mathrm{CH}), 119.3(\mathrm{q}, J=3.7$ $\mathrm{Hz}, \mathrm{CH}), 117.2$ (q, $J=3.7 \mathrm{~Hz}, \mathrm{CH}$ ).

${ }^{19} \mathrm{~F}$ NMR $(282 \mathrm{MHz}$, Chloroform- $d$ ) $\delta$-64.9.

HRMS (ESI-TOF) $m / z$ : $[\mathrm{M}+\mathrm{H}]^{+}$Calcd for $\mathrm{C}_{20} \mathrm{H}_{12} \mathrm{~F}_{3} \mathrm{~N}_{3}$ 352.1056; Found 352.1056. 


\section{NMR Spectra}

${ }^{1} \mathrm{H}$ NMR (400 MHz, $\mathrm{CDCl}_{3}$ ): $1 \mathrm{a}$

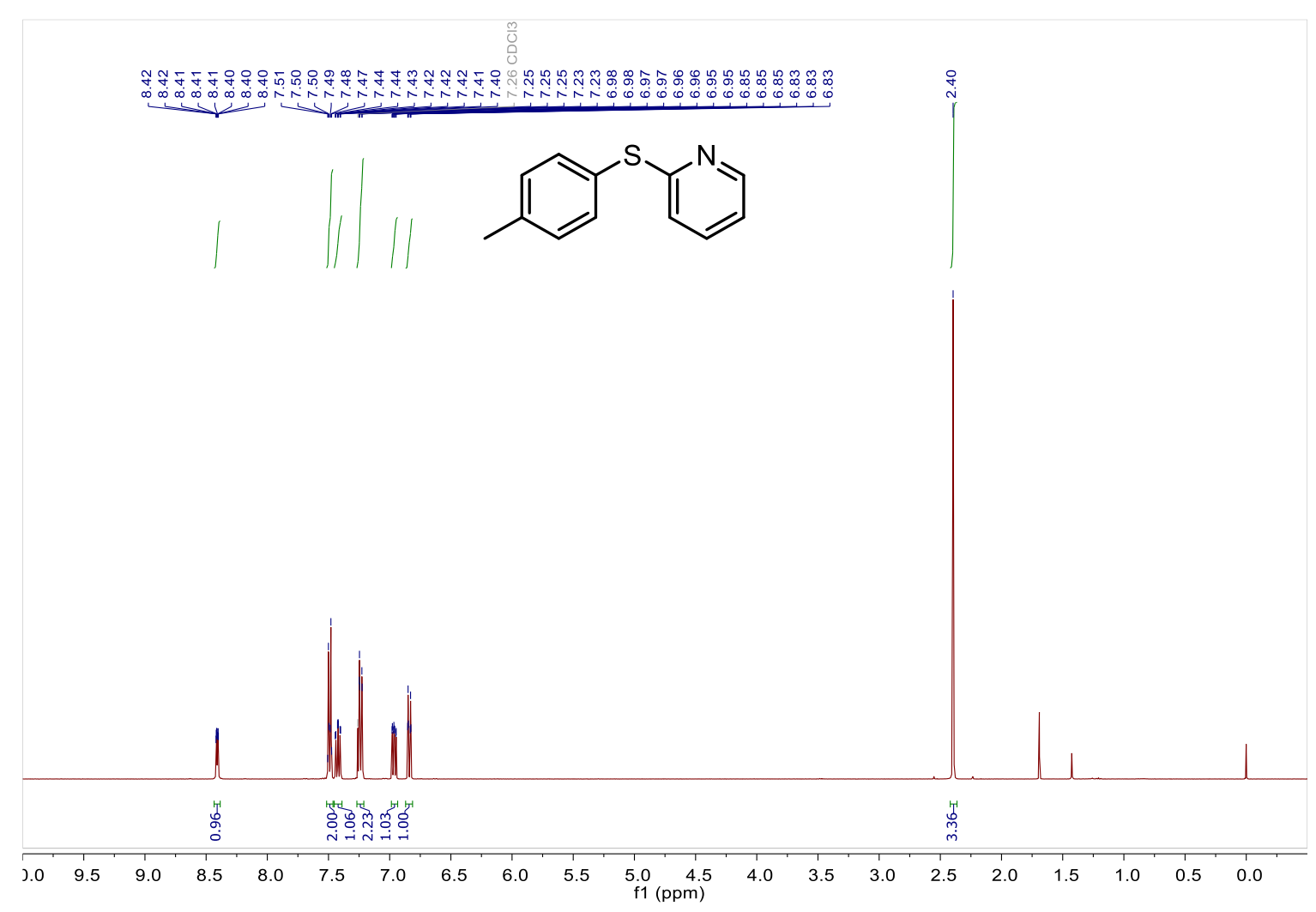

${ }^{13} \mathrm{C}$ NMR (101 MHz, $\left.\mathrm{CDCl}_{3}\right): 1 \mathrm{a}$

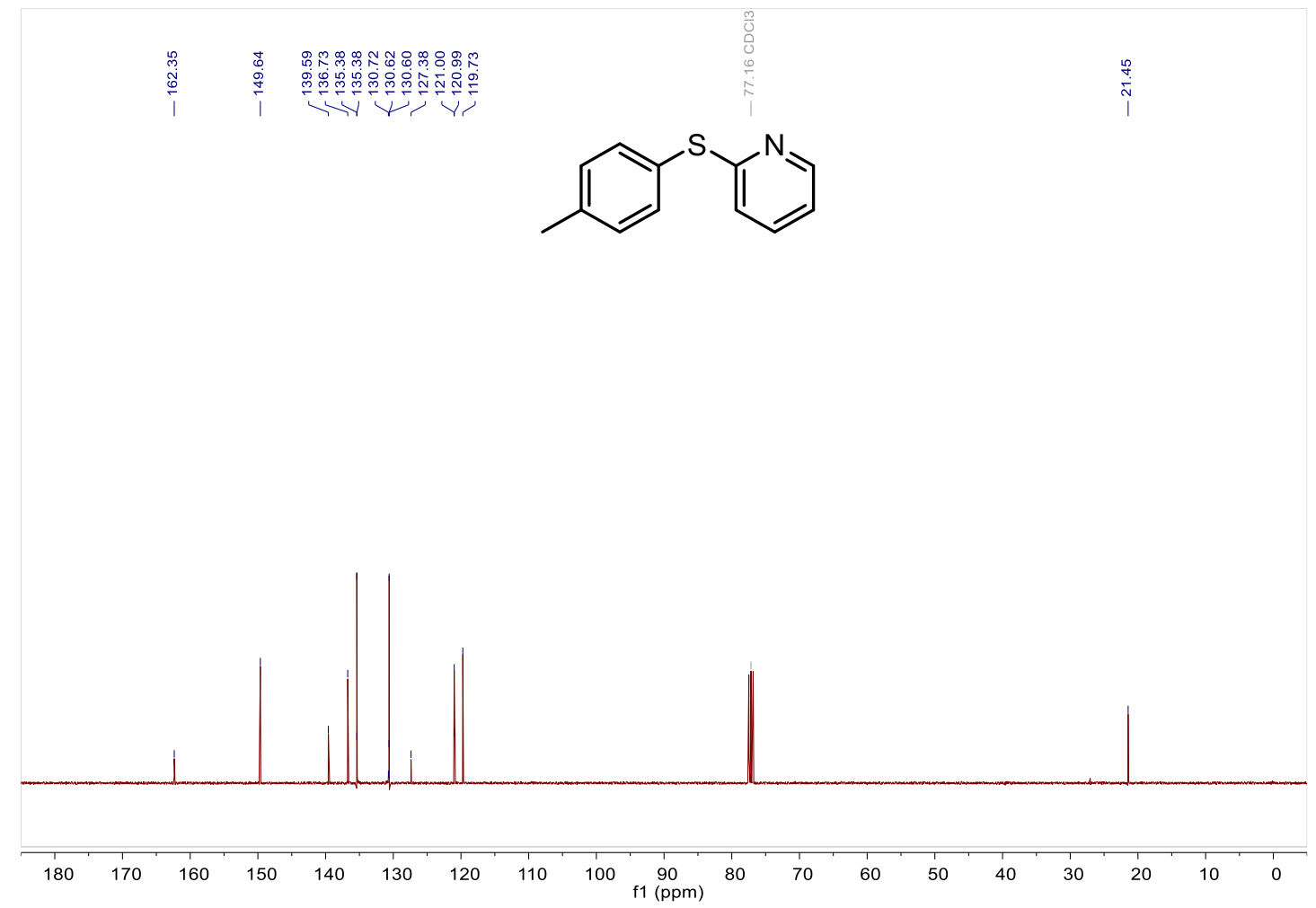


${ }^{1} \mathrm{H}$ NMR (400 MHz, $\mathrm{CDCl}_{3}$ ): 1b

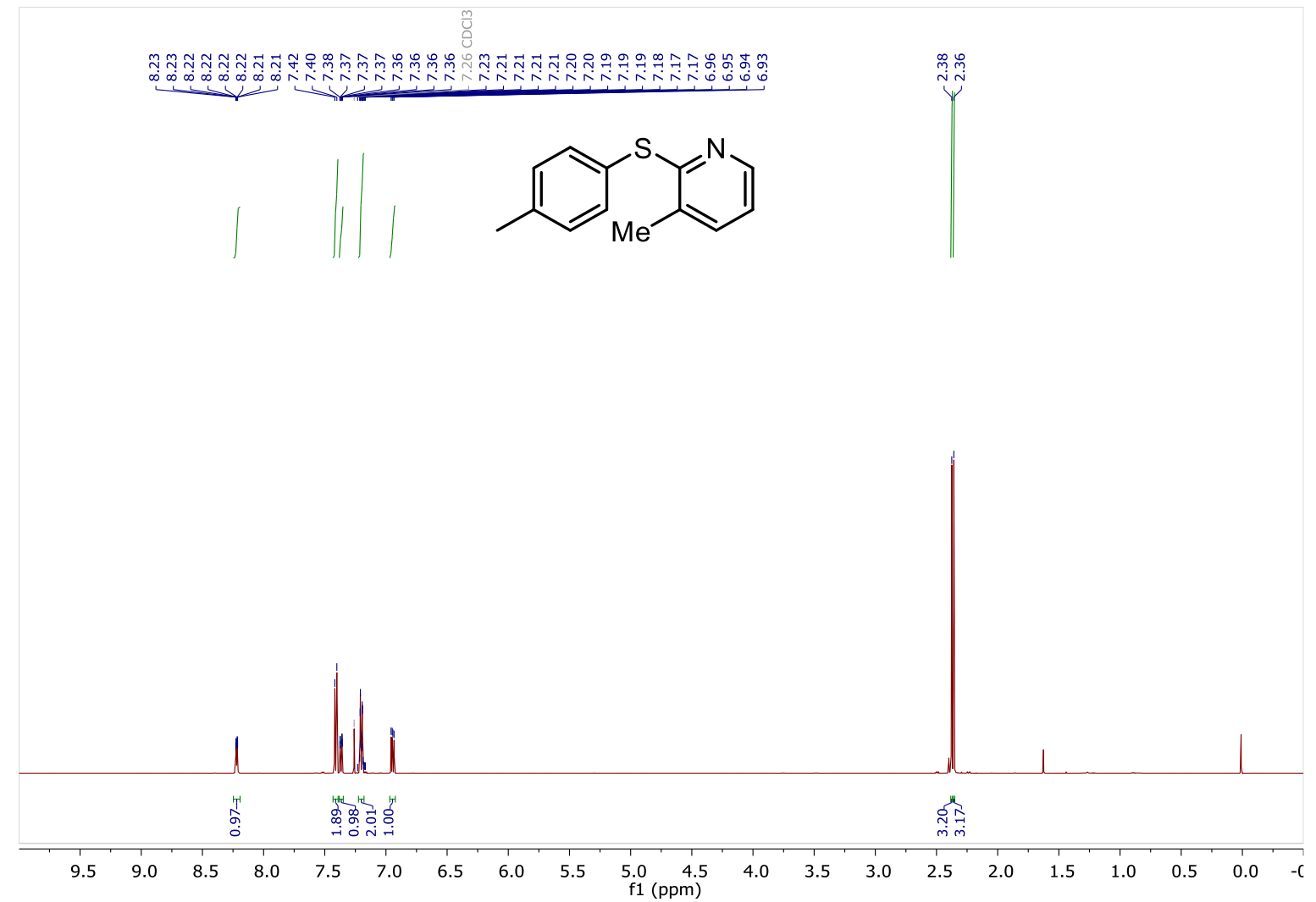

${ }^{13} \mathrm{C}$ NMR (101 MHz, $\left.\mathrm{CDCl}_{3}\right)$ : 1b

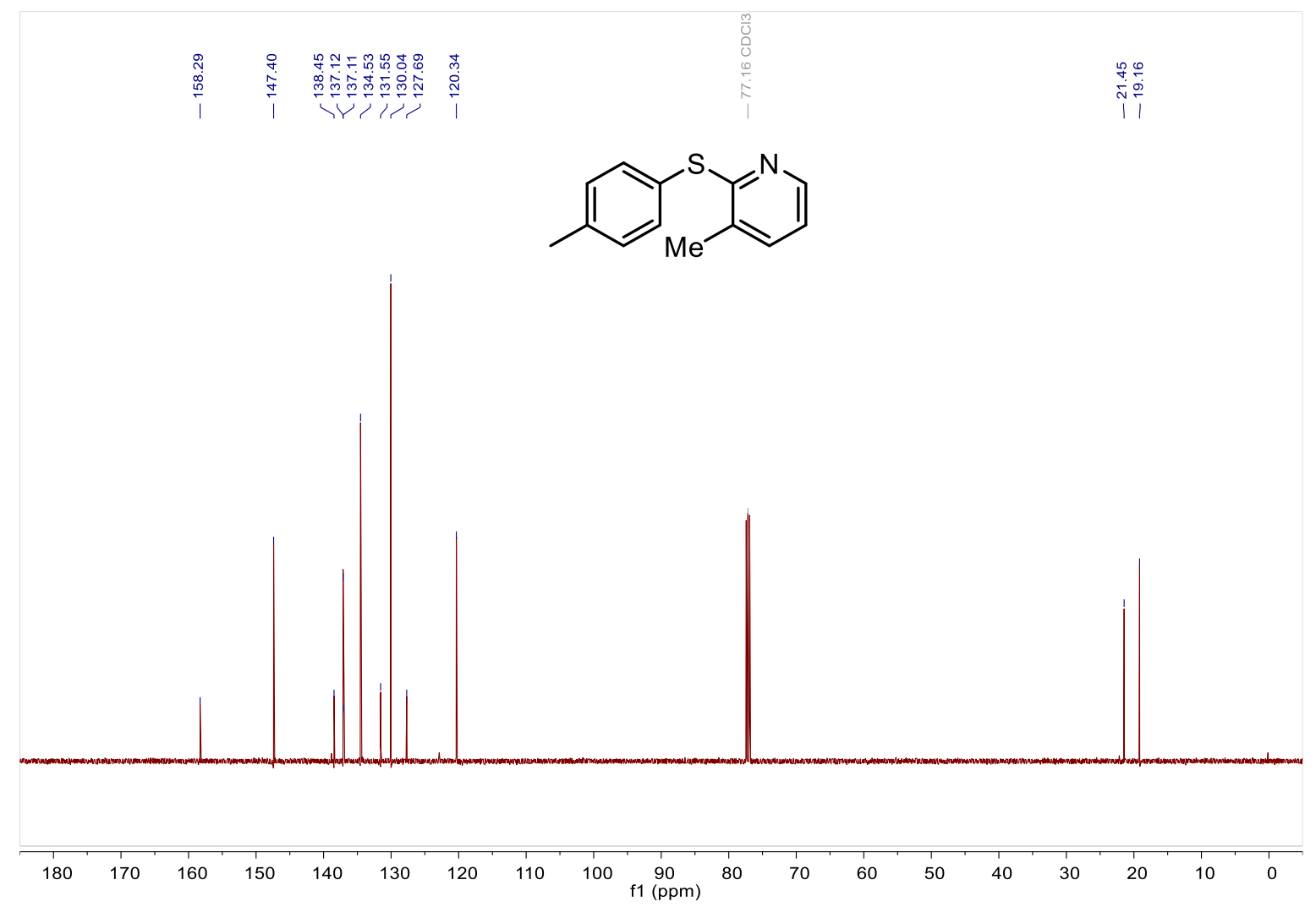


${ }^{1} \mathrm{H}$ NMR (500 MHz, $\mathrm{CDCl}_{3}$ ): $1 \mathrm{c}$

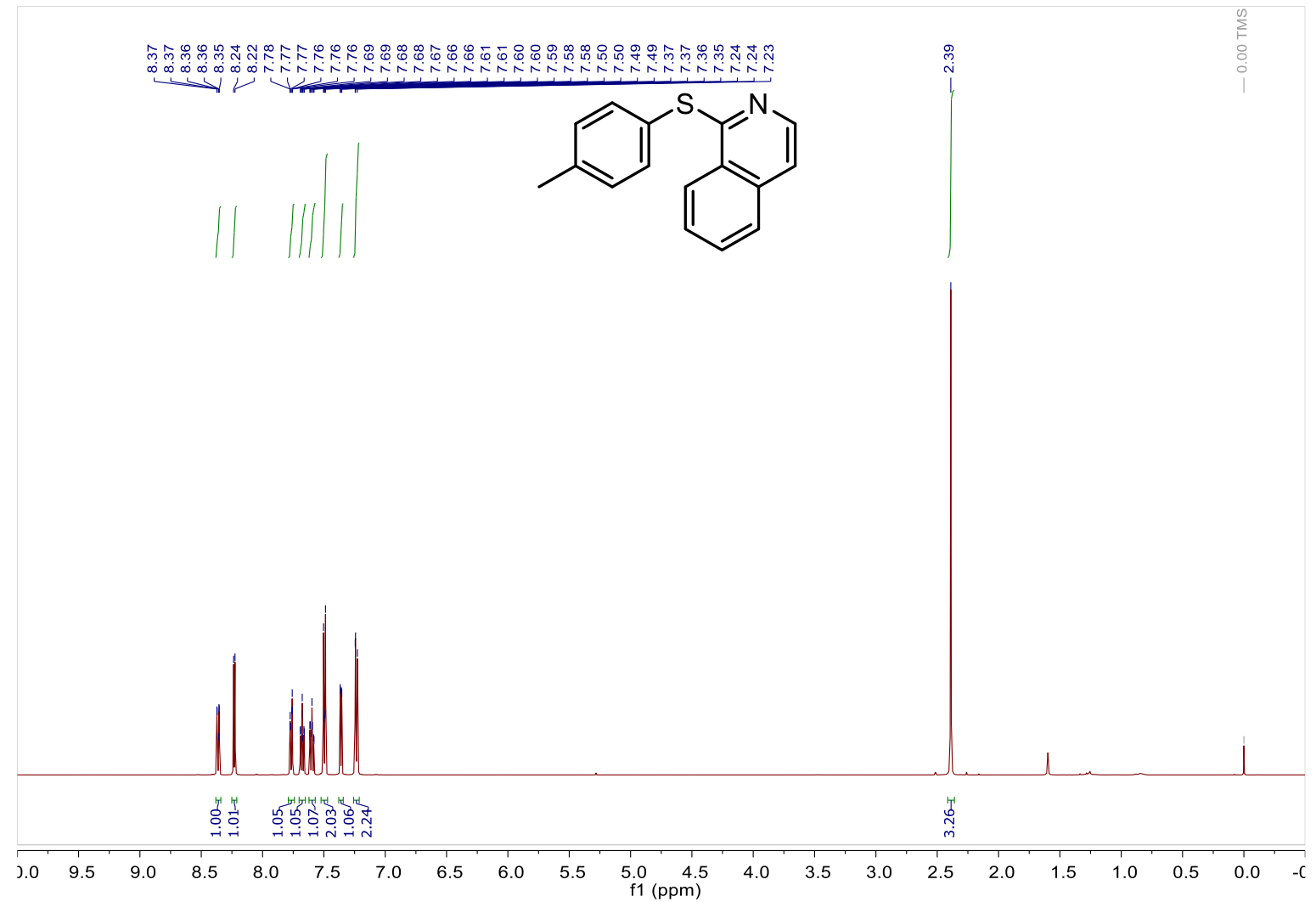

${ }^{13} \mathrm{C}$ NMR (126 MHz, $\mathrm{CDCl}_{3}$ ): $1 \mathrm{c}$

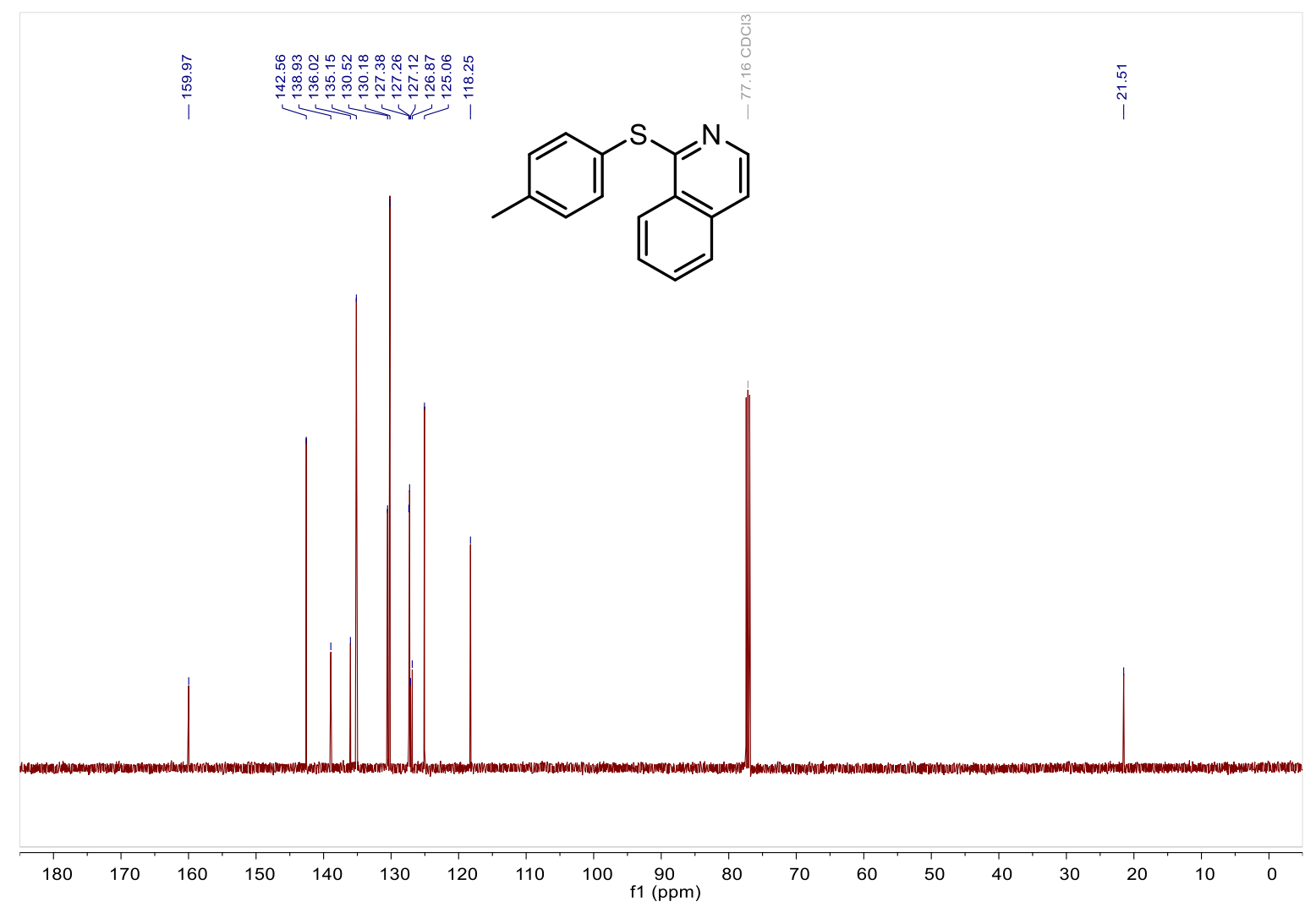


${ }^{1} \mathrm{H}$ NMR (500 MHz, $\mathrm{CDCl}_{3}$ ): 1d

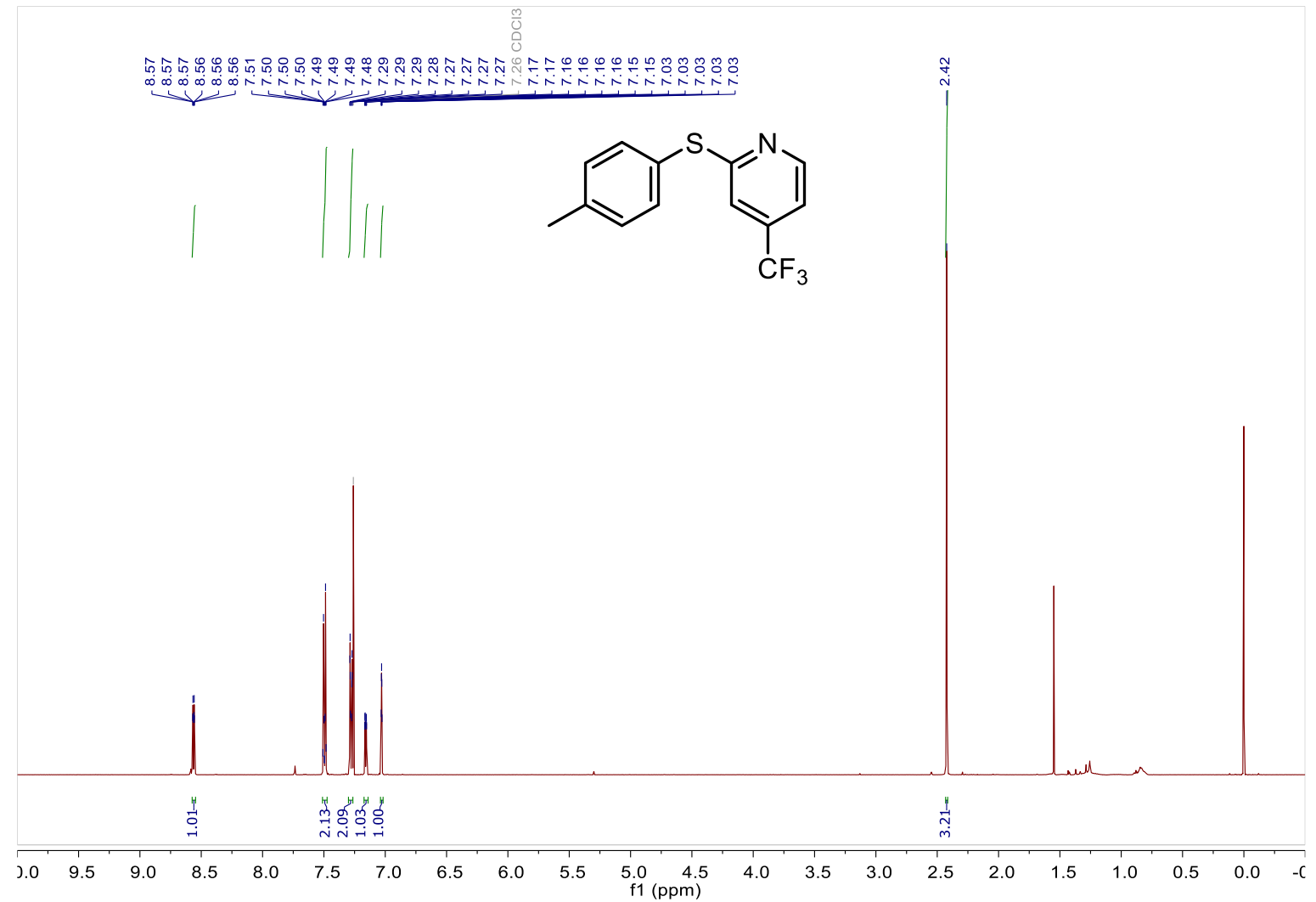

${ }^{13}$ C NMR (126 MHz, $\left.\mathrm{CDCl}_{3}\right): 1 \mathrm{~d}$

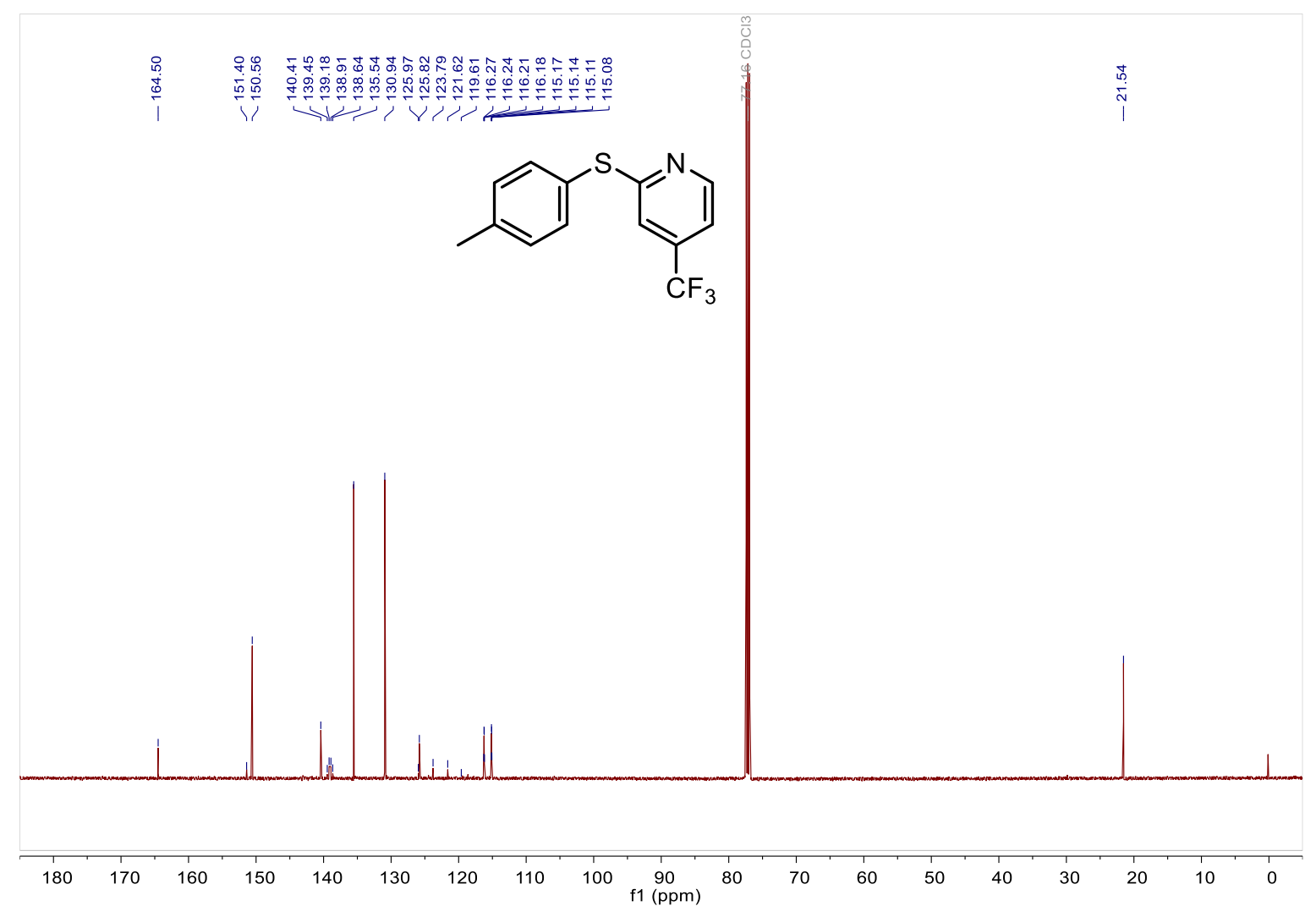


${ }^{1} \mathrm{H}$ NMR (500 MHz, $\mathrm{CDCl}_{3}$ ): $1 \mathrm{e}$

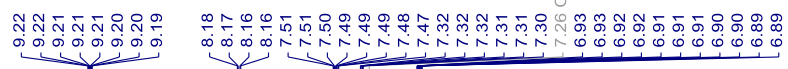

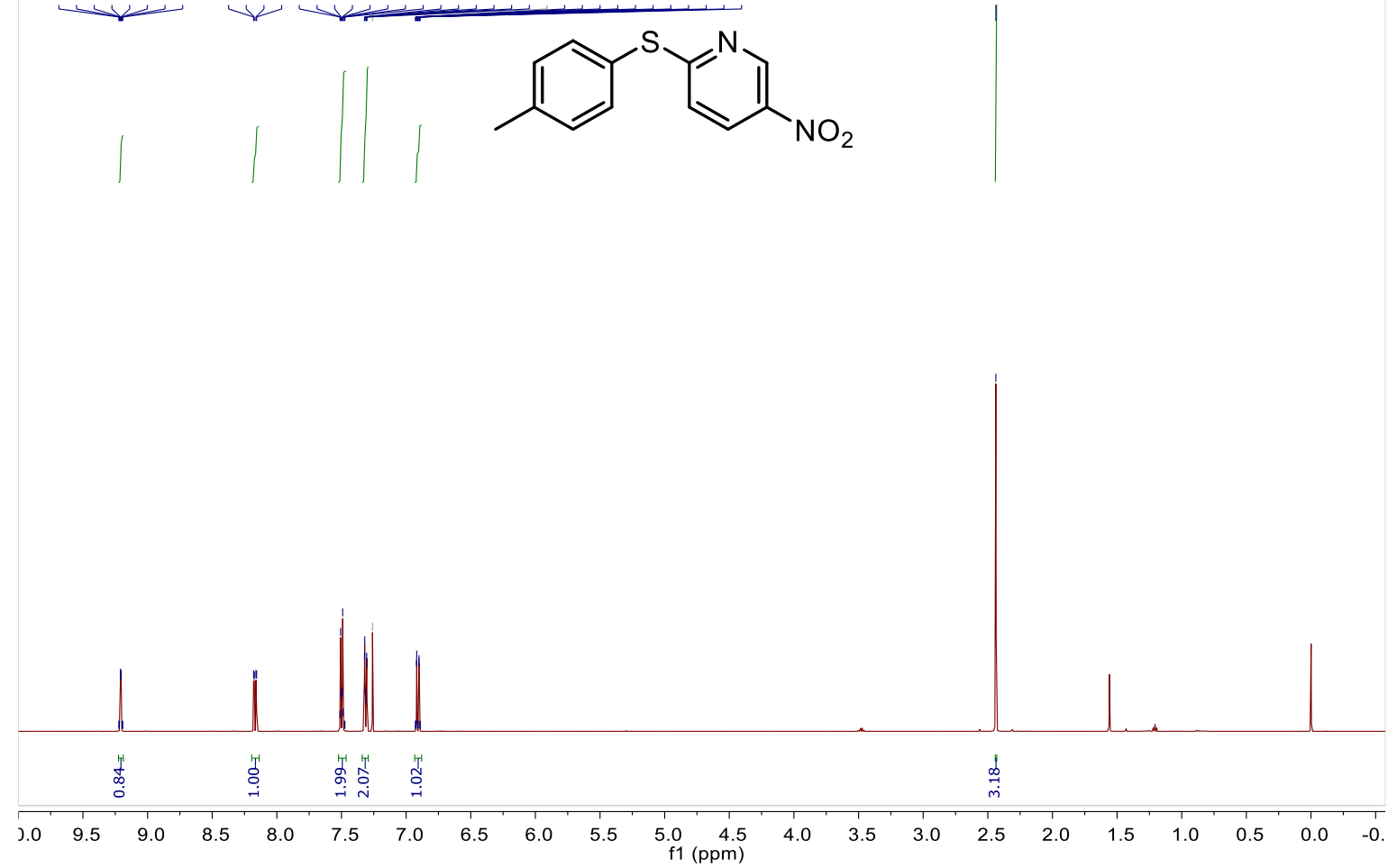

${ }^{13} \mathrm{C}$ NMR (126 MHz, $\mathrm{CDCl}_{3}$ ): $1 \mathrm{e}$

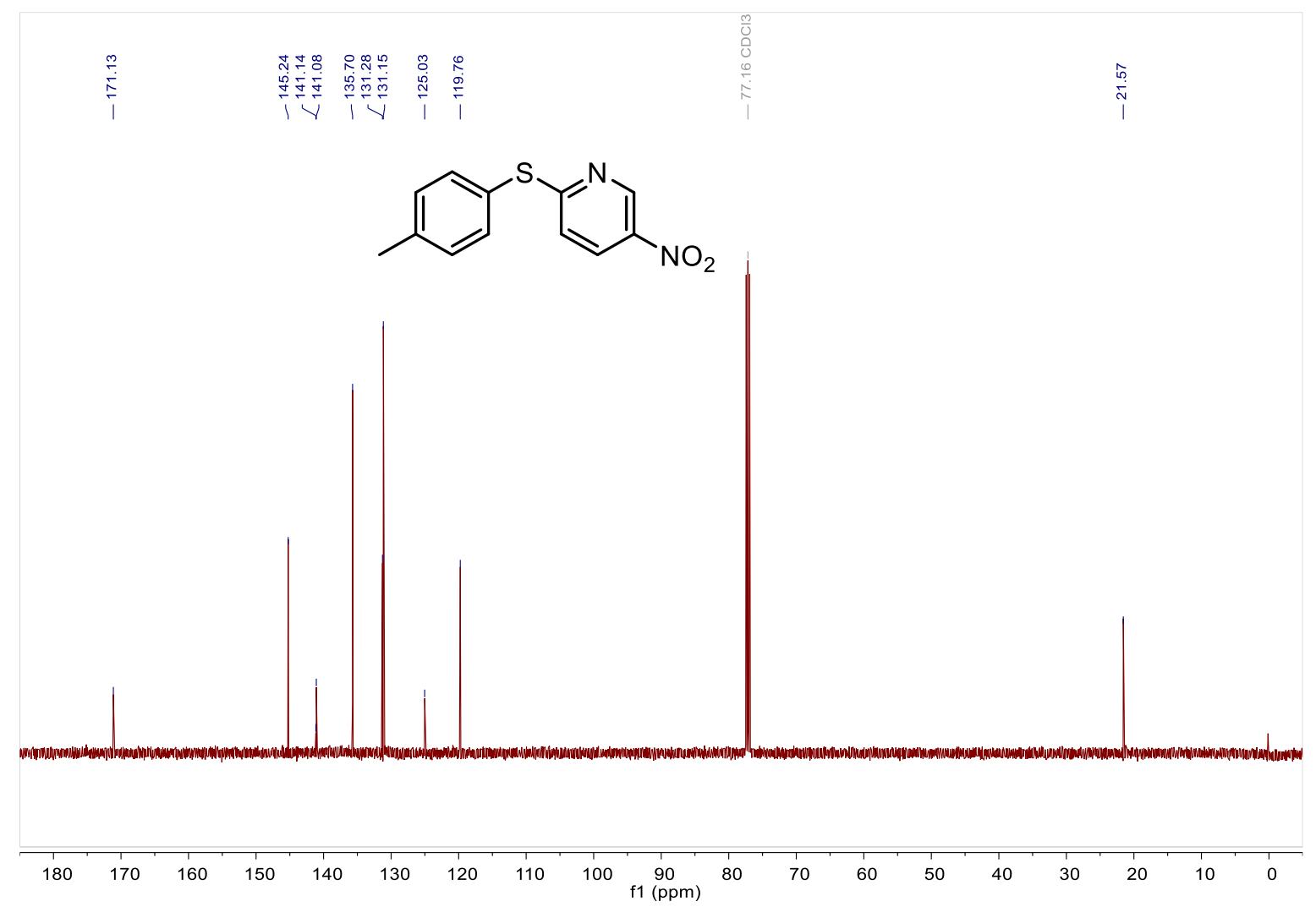


${ }^{1} \mathrm{H}$ NMR (500 MHz, $\mathrm{CDCl}_{3}$ ): 1f

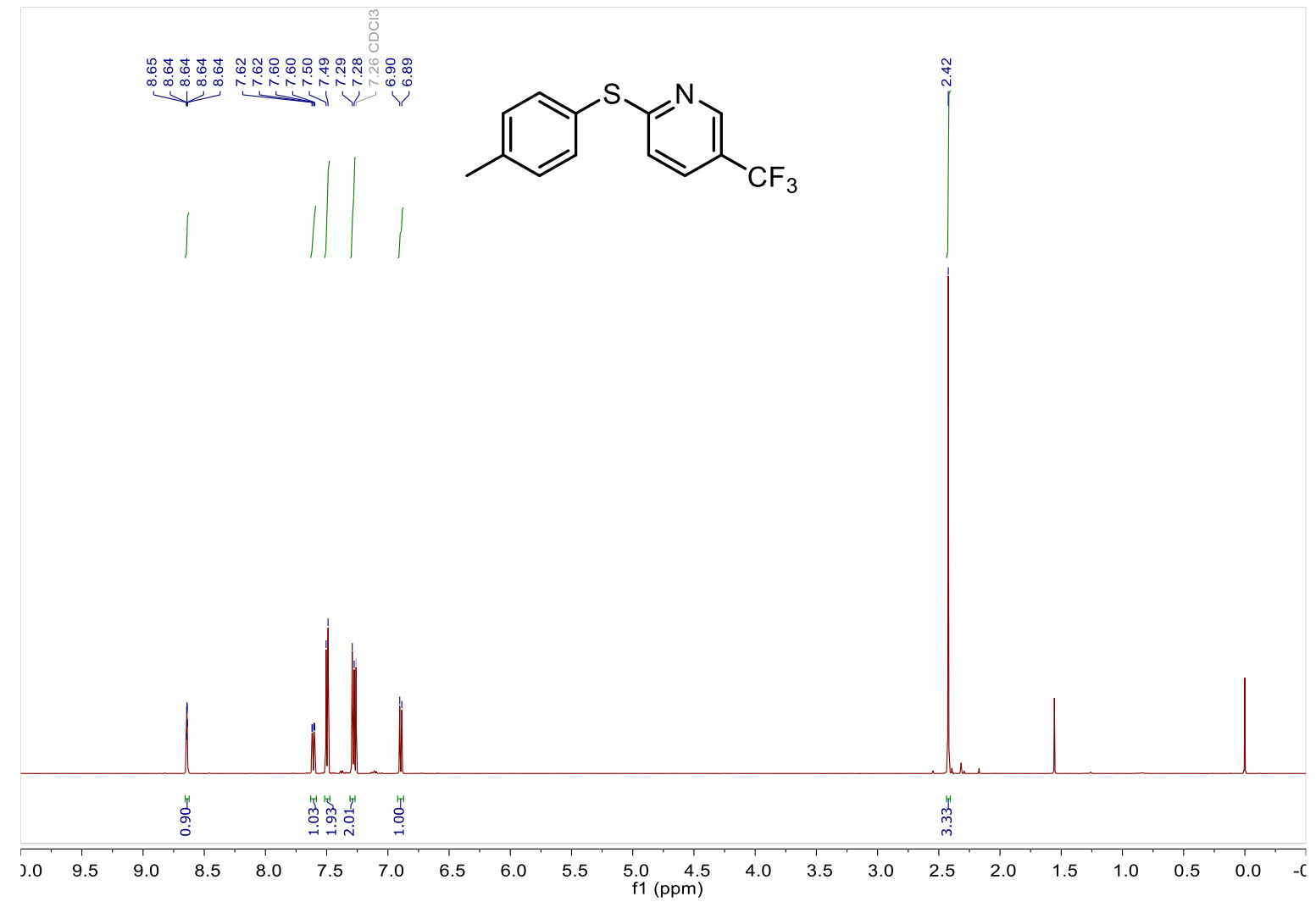

${ }^{13} \mathrm{C}$ NMR (101 MHz, $\mathrm{CDCl}_{3}$ ): 1f

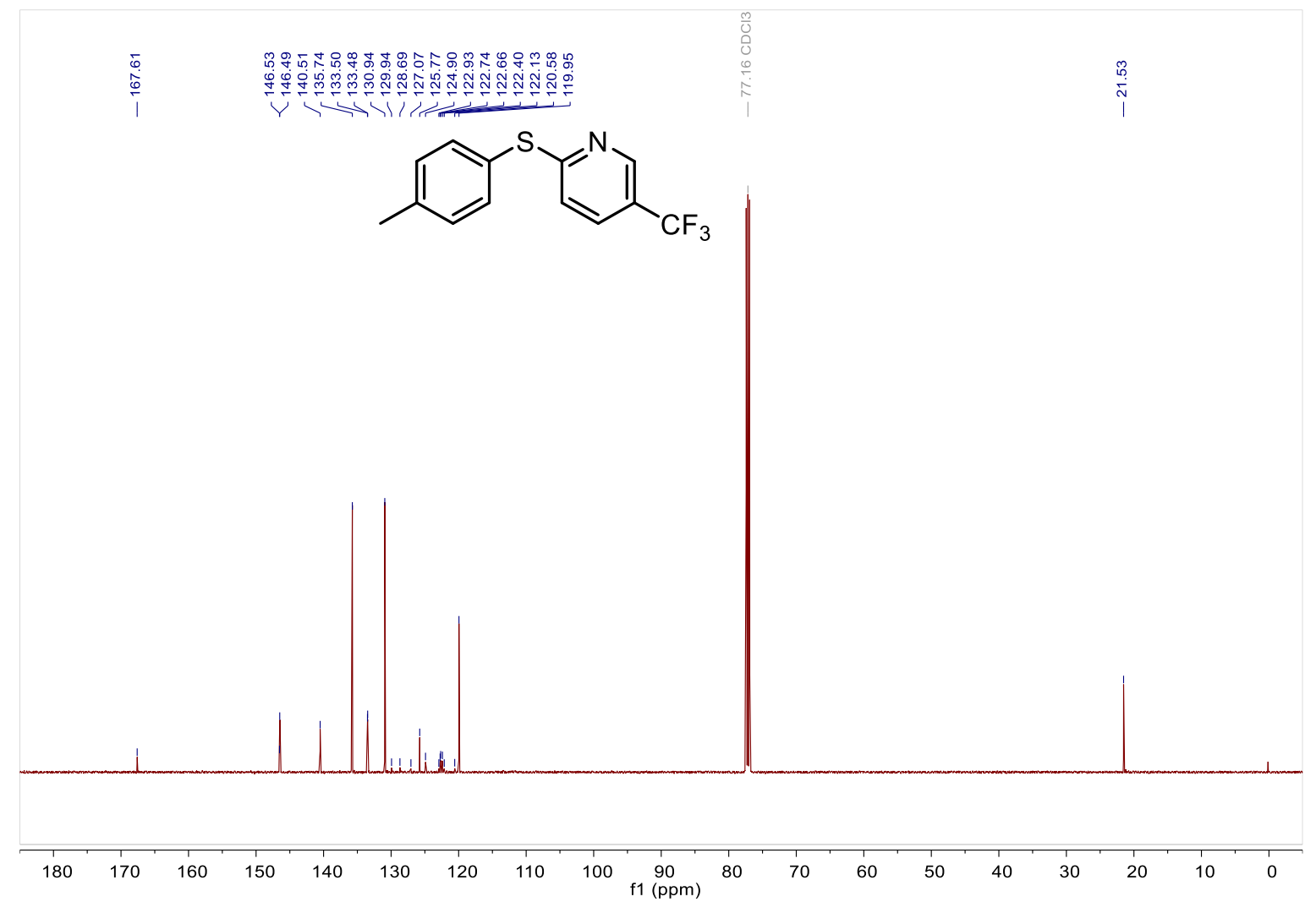


${ }^{1}$ H NMR (500 MHz, $\left.\mathrm{CDCl}_{3}\right): 1 \mathrm{~g}$

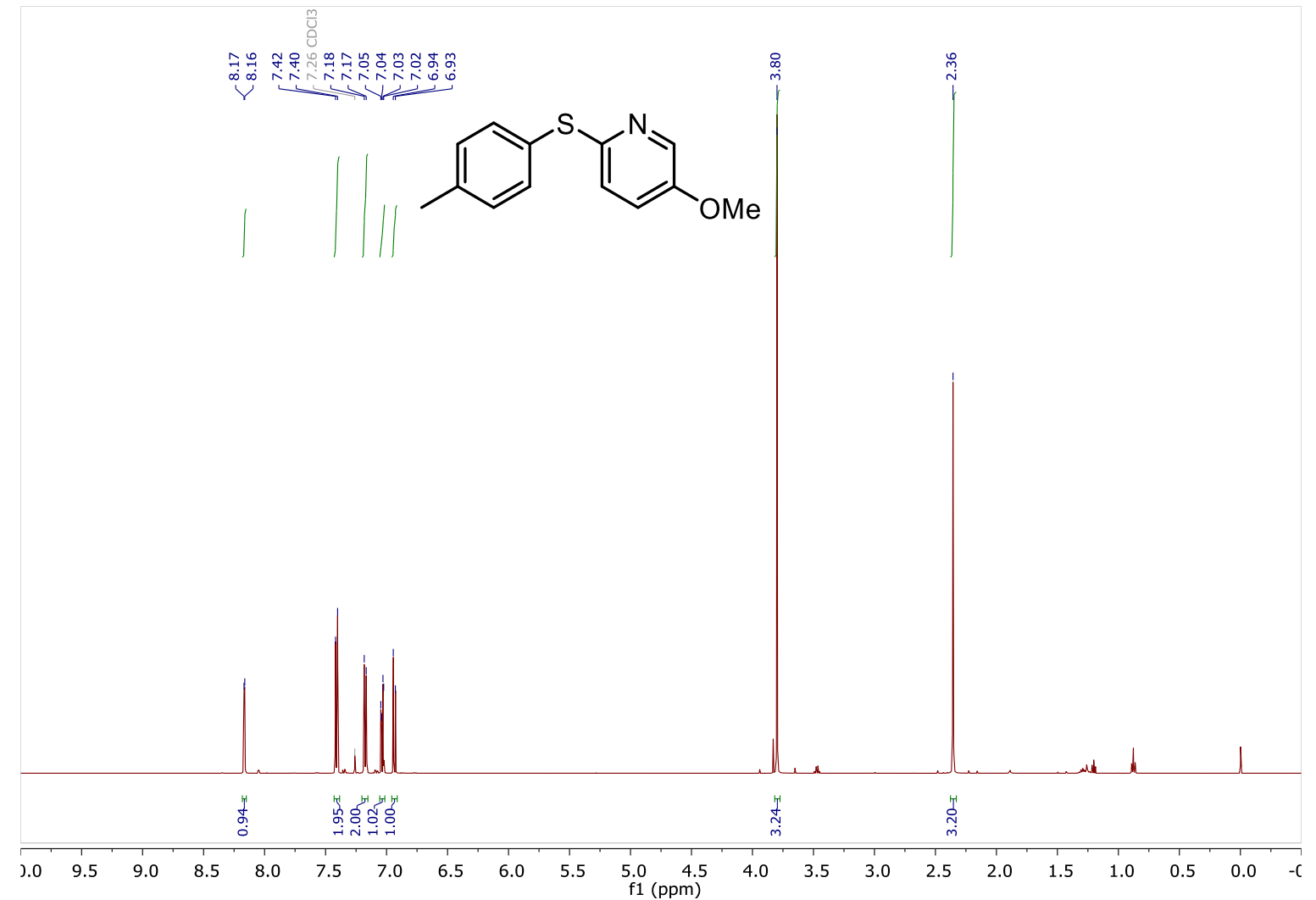

${ }^{13} \mathrm{C}$ NMR (126 MHz, $\left.\mathrm{CDCl}_{3}\right): 1 \mathrm{~g}$

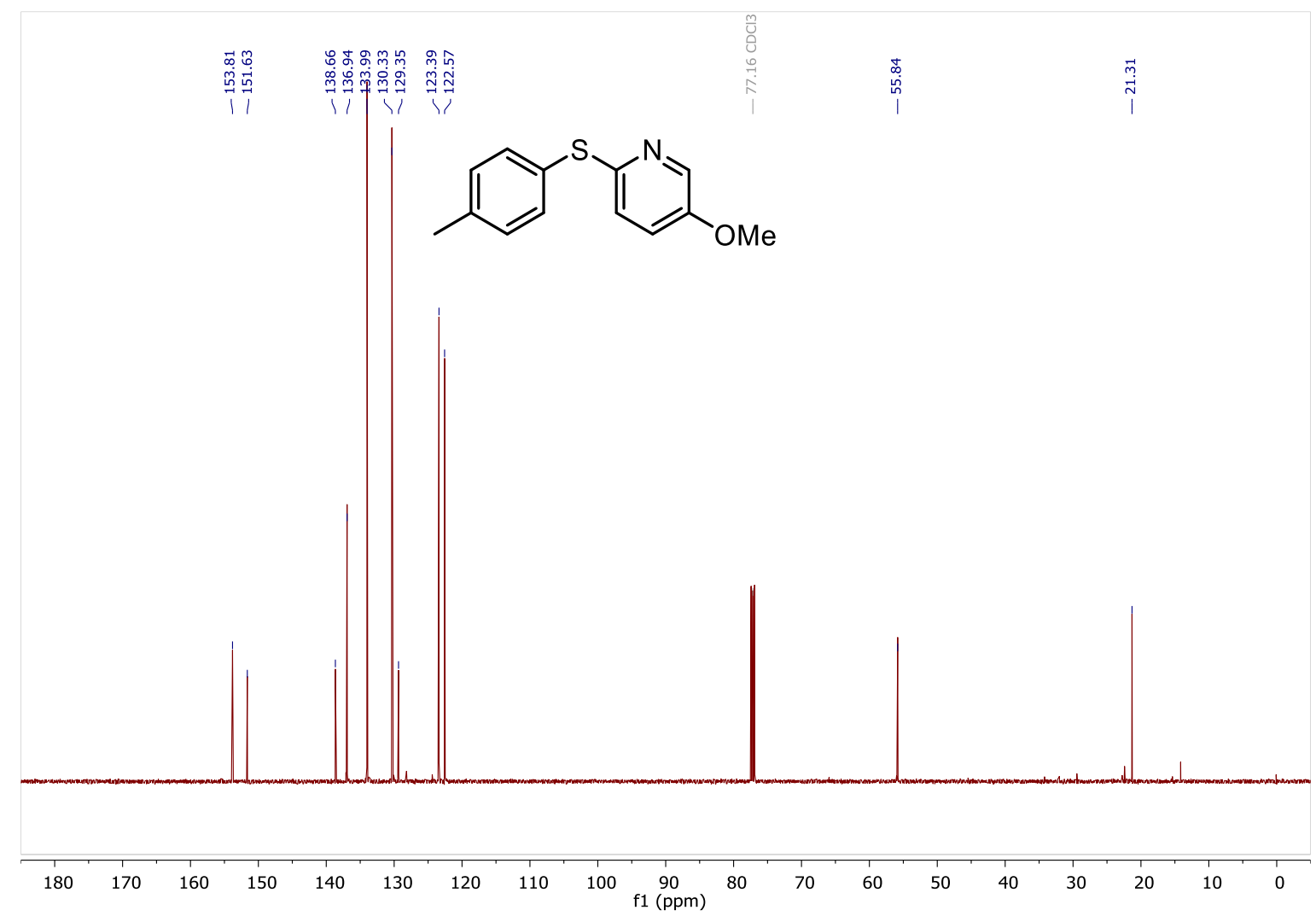


${ }^{1} \mathrm{H}$ NMR (500 MHz, $\mathrm{CDCl}_{3}$ ): $1 \mathrm{~h}$

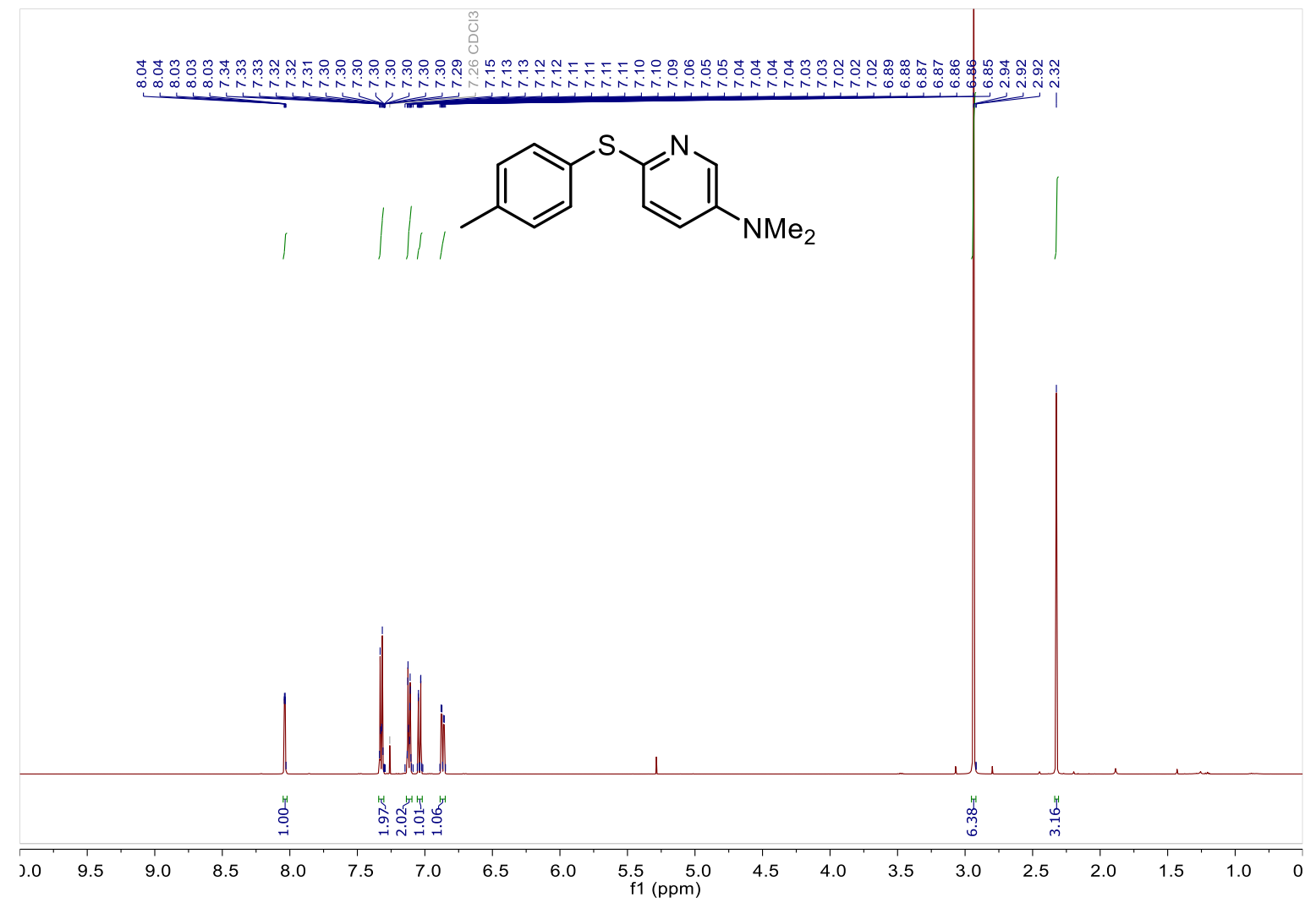

${ }^{13} \mathrm{C}$ NMR (126 MHz, $\left.\mathrm{CDCl}_{3}\right): 1 \mathrm{~h}$

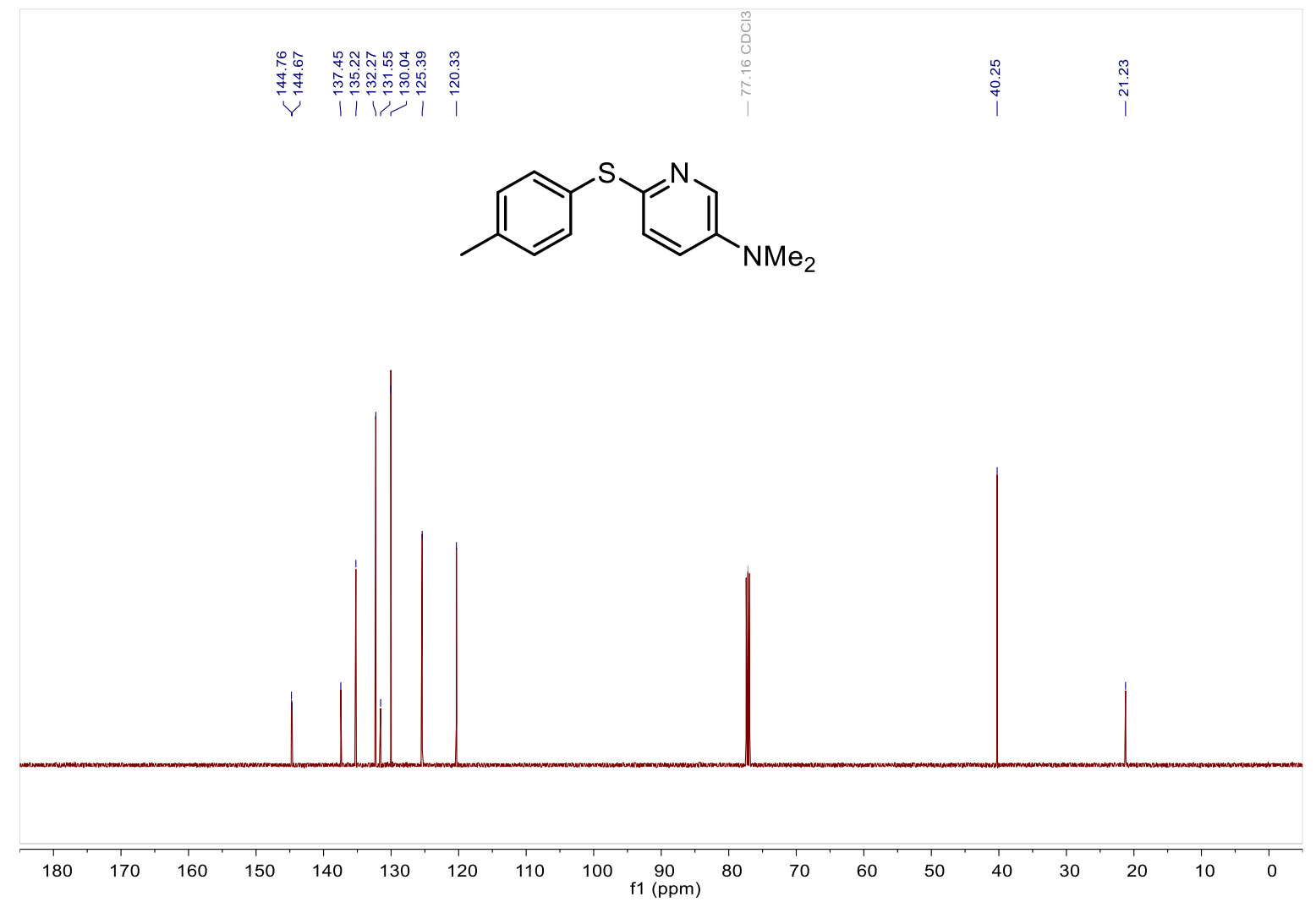


${ }^{1} \mathrm{H}$ NMR (500 MHz, $\mathrm{CDCl}_{3}$ ): 1i

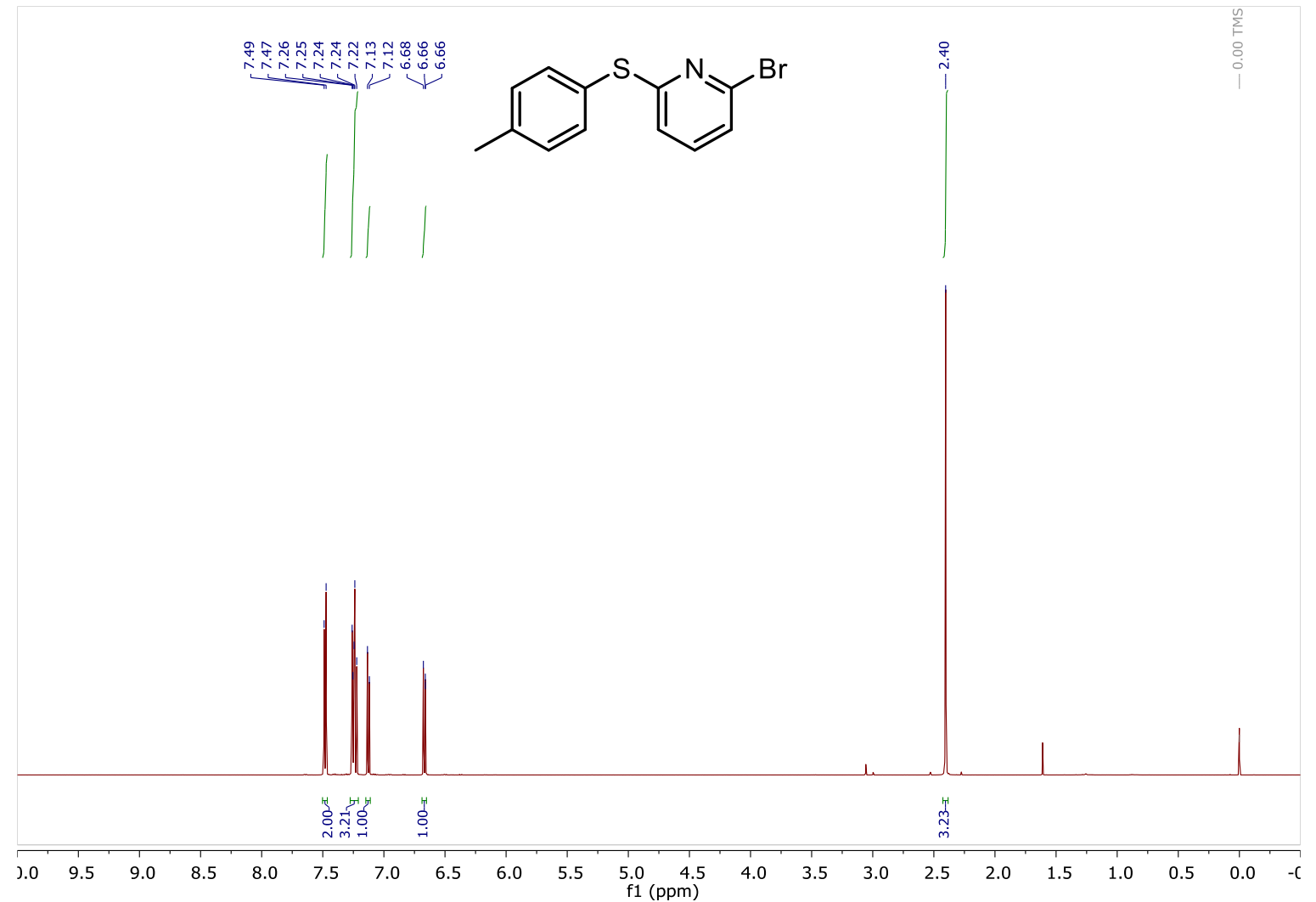

${ }^{13} \mathrm{C}$ NMR (126 MHz, $\mathrm{CDCl}_{3}$ ): 1i

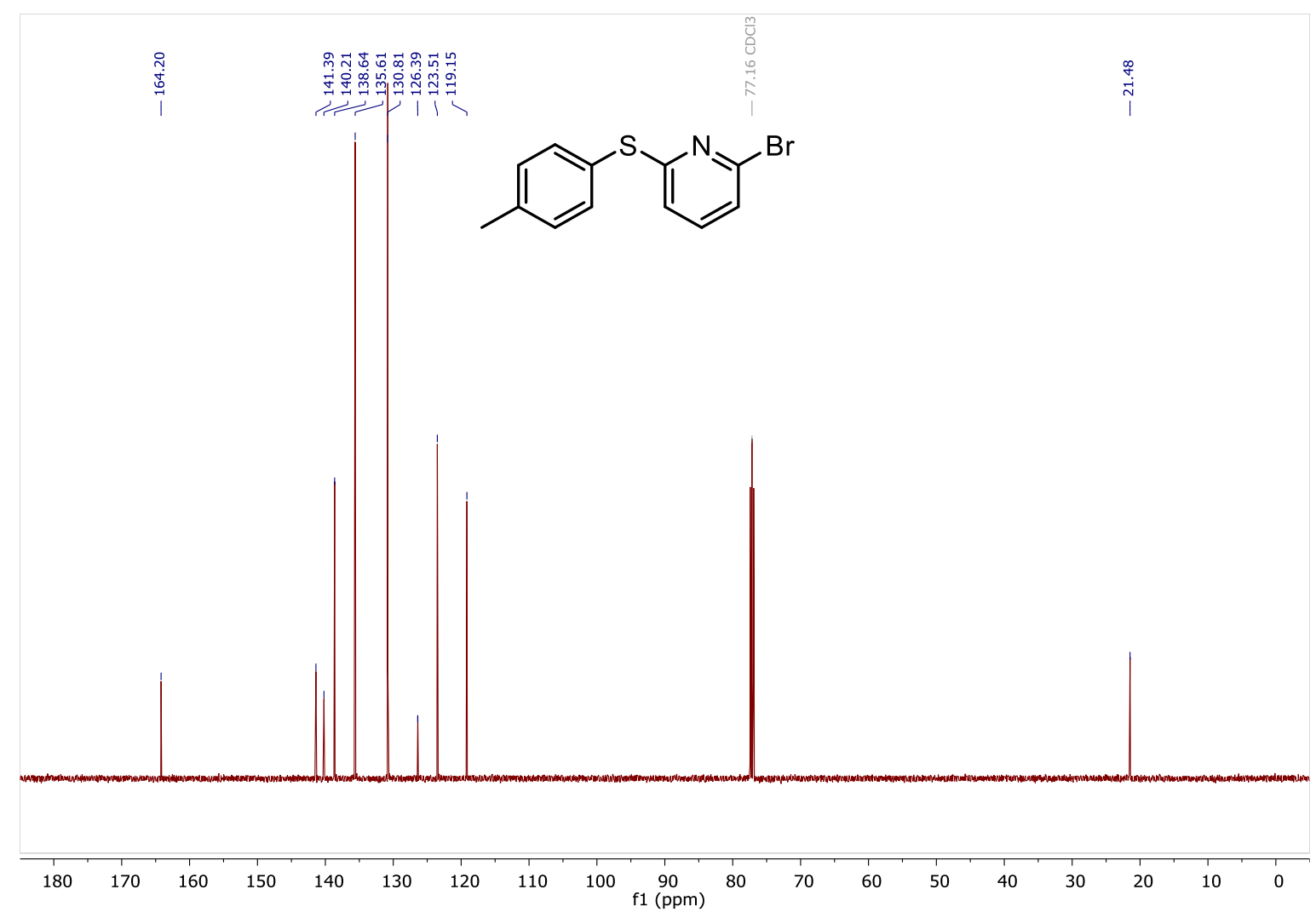


${ }^{1} \mathrm{H}$ NMR (400 MHz, $\mathrm{CDCl}_{3}$ ): Z-S1

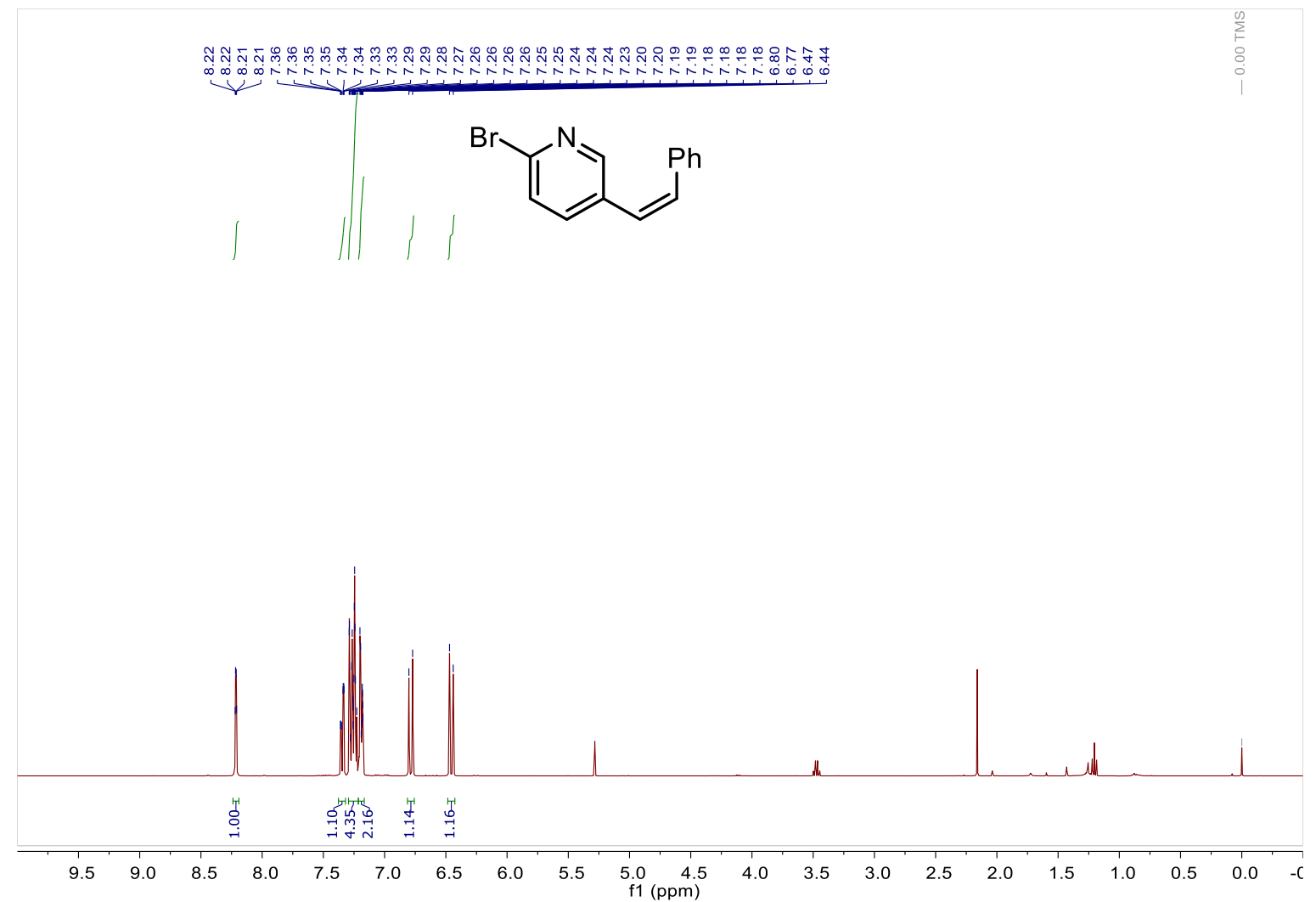

${ }^{13}$ C NMR (101 MHz, $\left.\mathrm{CDCl}_{3}\right): Z-S 1$

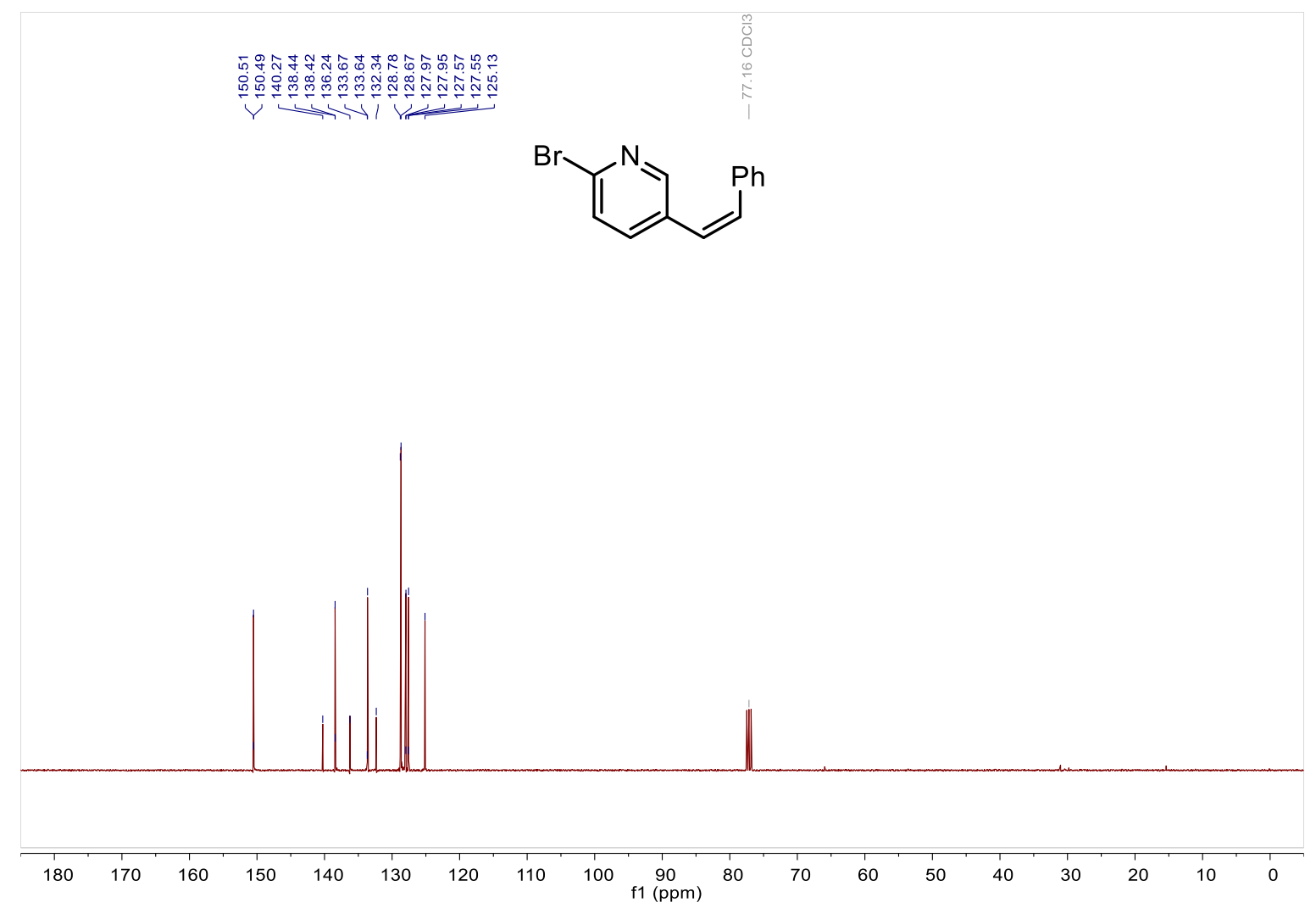


${ }^{1} \mathrm{H}$ NMR (400 MHz, $\mathrm{CDCl}_{3}$ ): $E-\mathrm{S} 1$

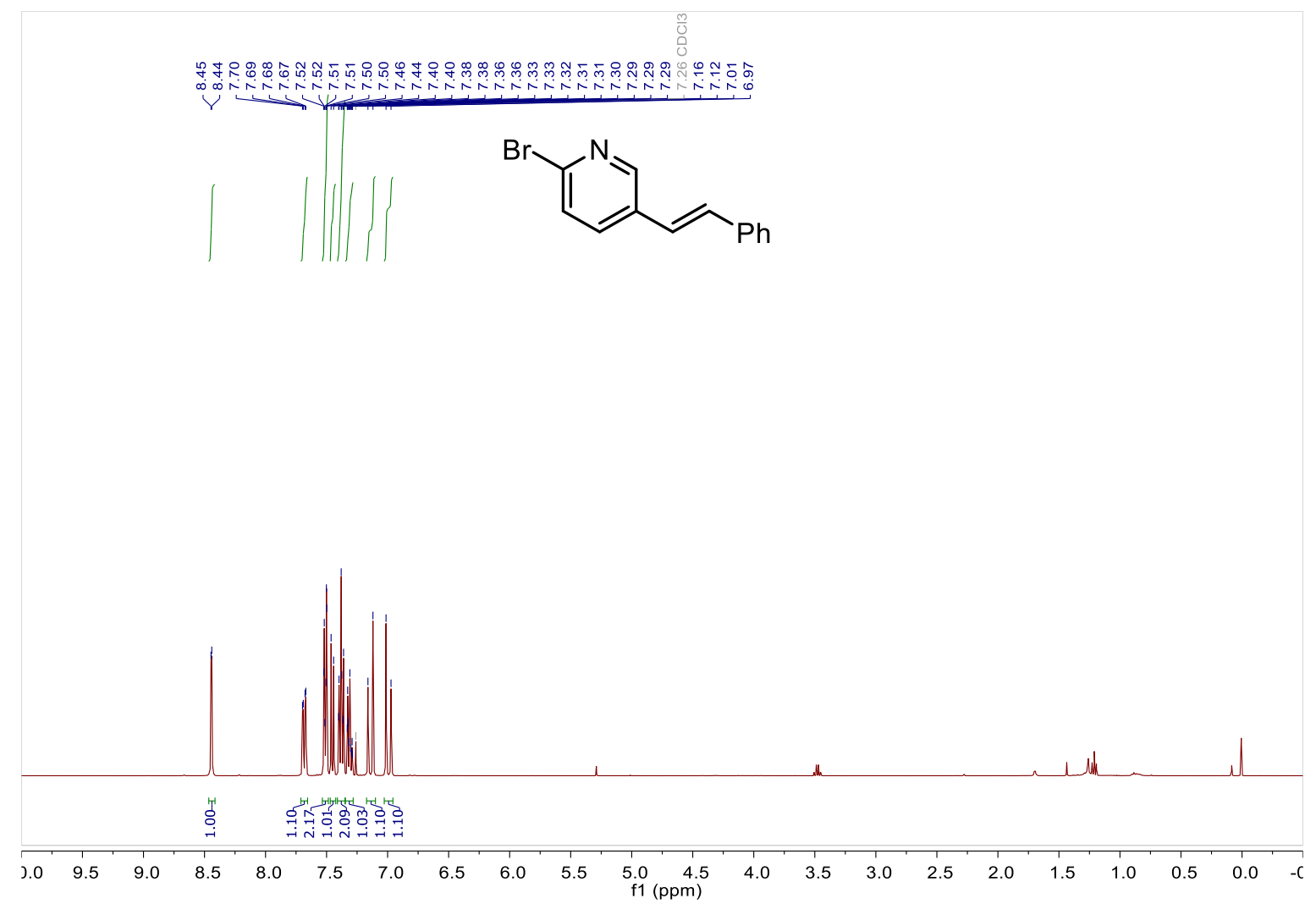

${ }^{13} \mathrm{C}$ NMR (101 MHz, $\left.\mathrm{CDCl}_{3}\right): E-\mathrm{S1}$

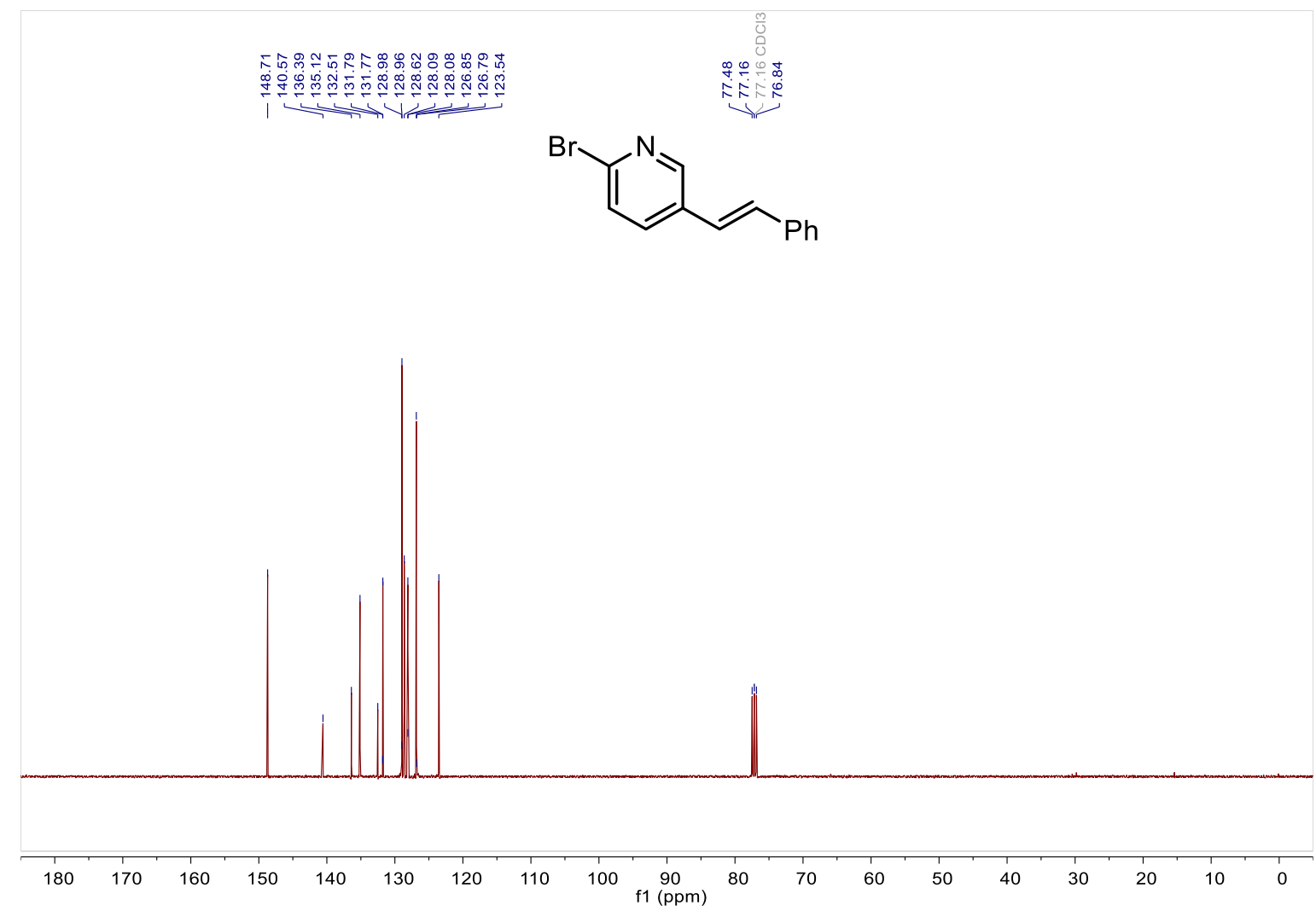


${ }^{1} \mathrm{H}$ NMR (400 MHz, $\mathrm{CDCl}_{3}$ ): $2 \mathrm{a}$

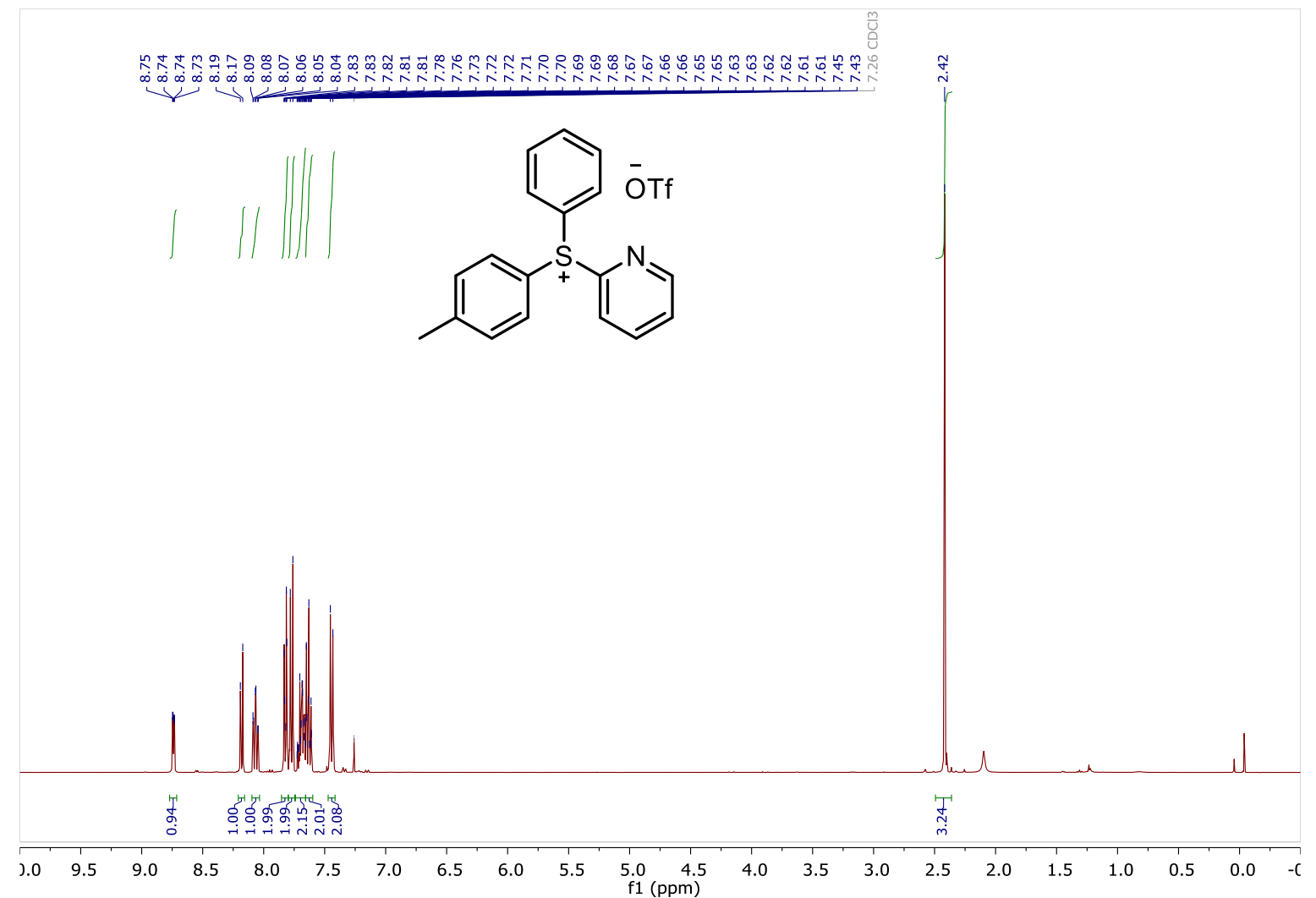

${ }^{13}$ C NMR (101 MHz, $\left.\mathrm{CDCl}_{3}\right): 2 \mathrm{a}$

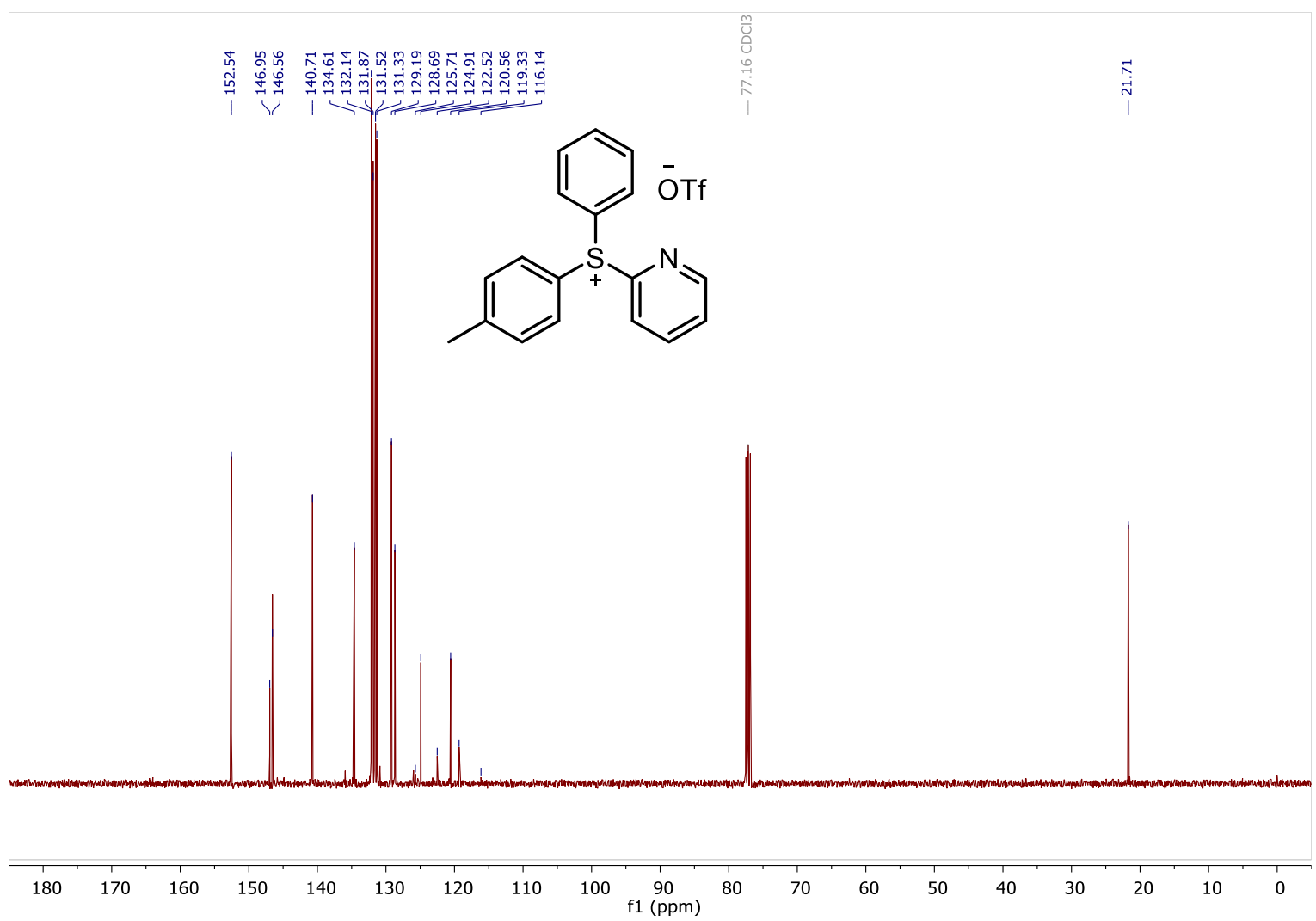


${ }^{1} \mathrm{H}$ NMR (400 MHz, $\mathrm{CDCl}_{3}$ ): 2b

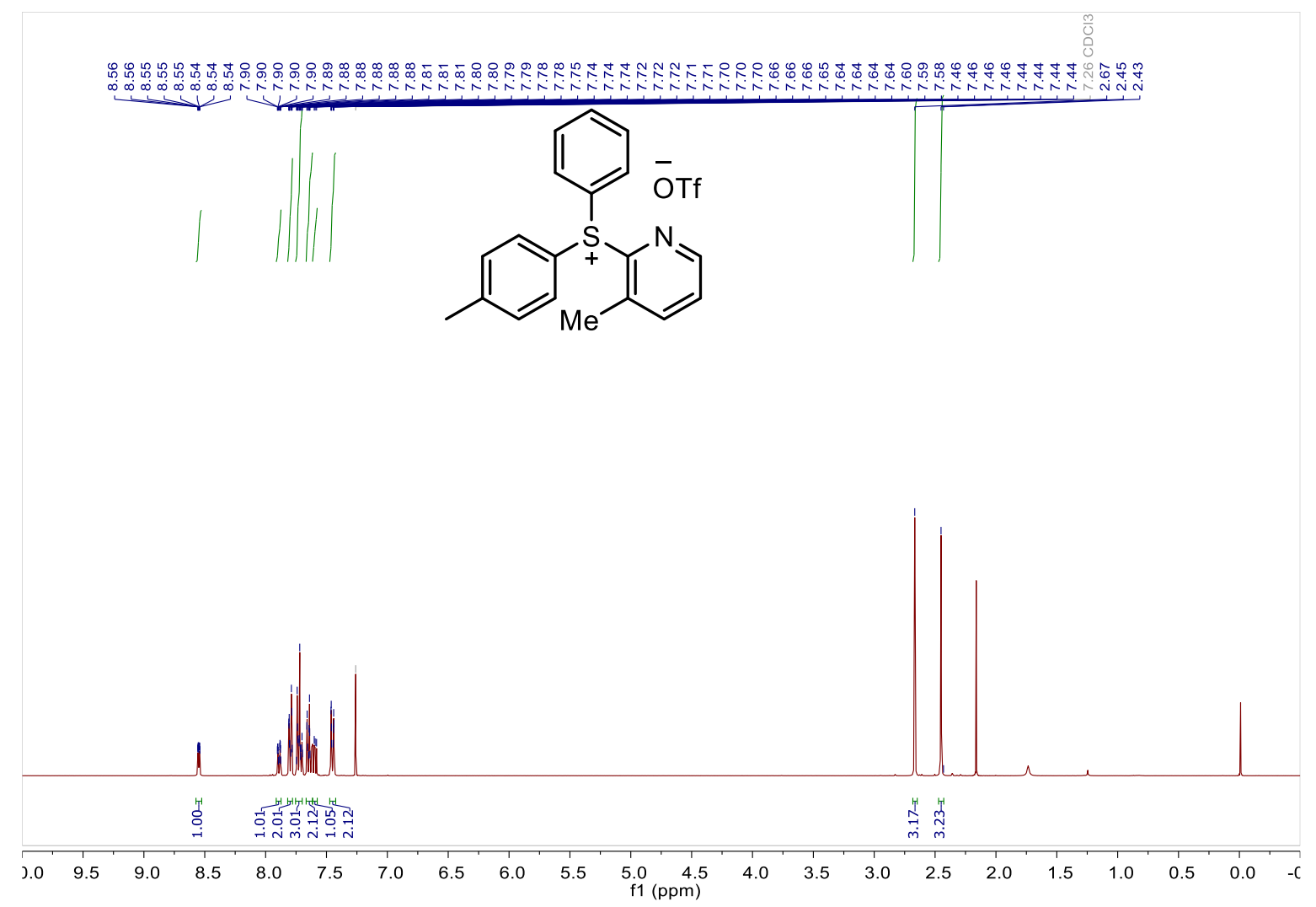

${ }^{13} \mathrm{C}$ NMR (101 MHz, $\left.\mathrm{CDCl}_{3}\right):$ 2b

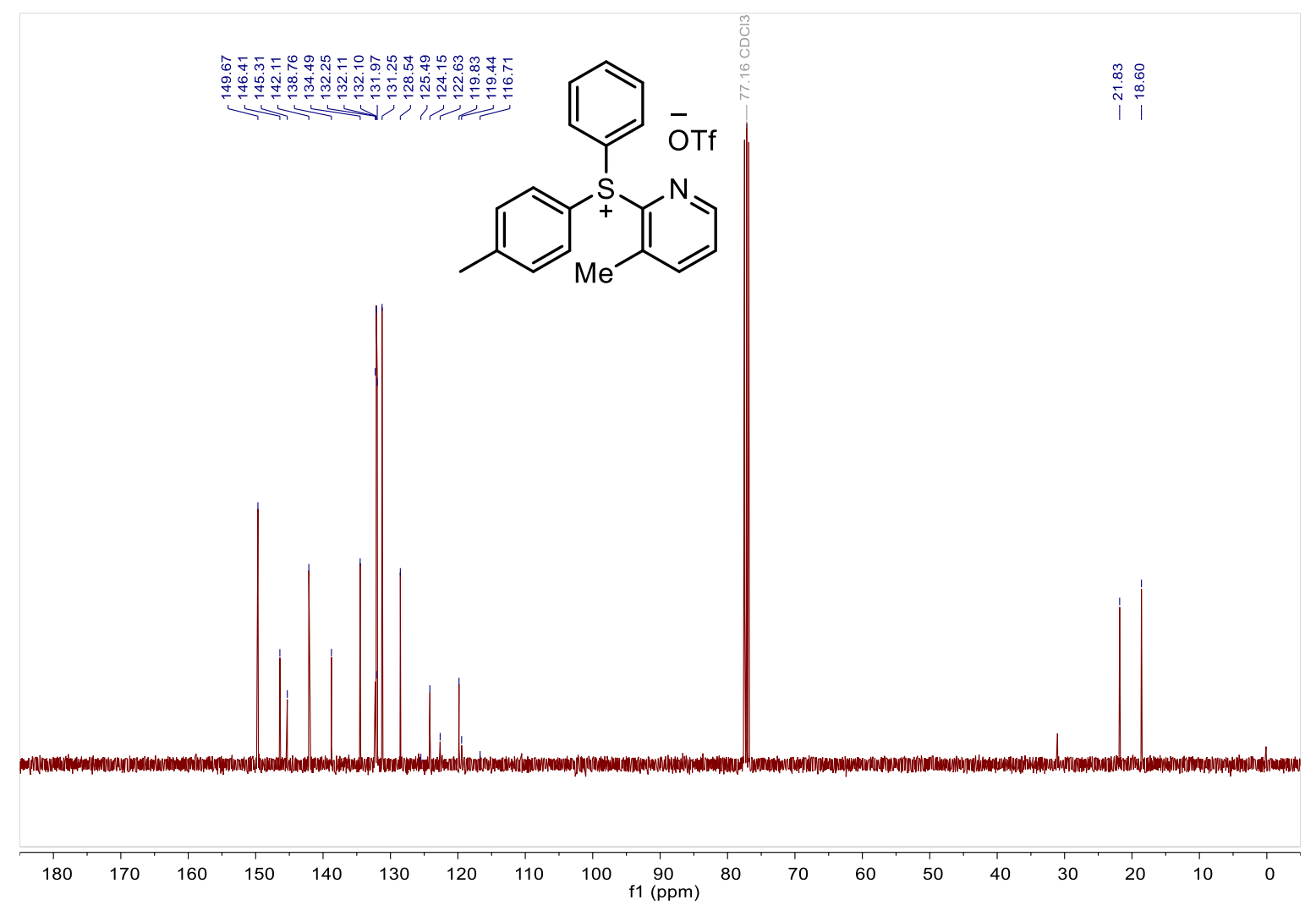


${ }^{1} \mathrm{H}$ NMR (500 MHz, $\mathrm{CDCl}_{3}$ ): $2 \mathrm{c}$

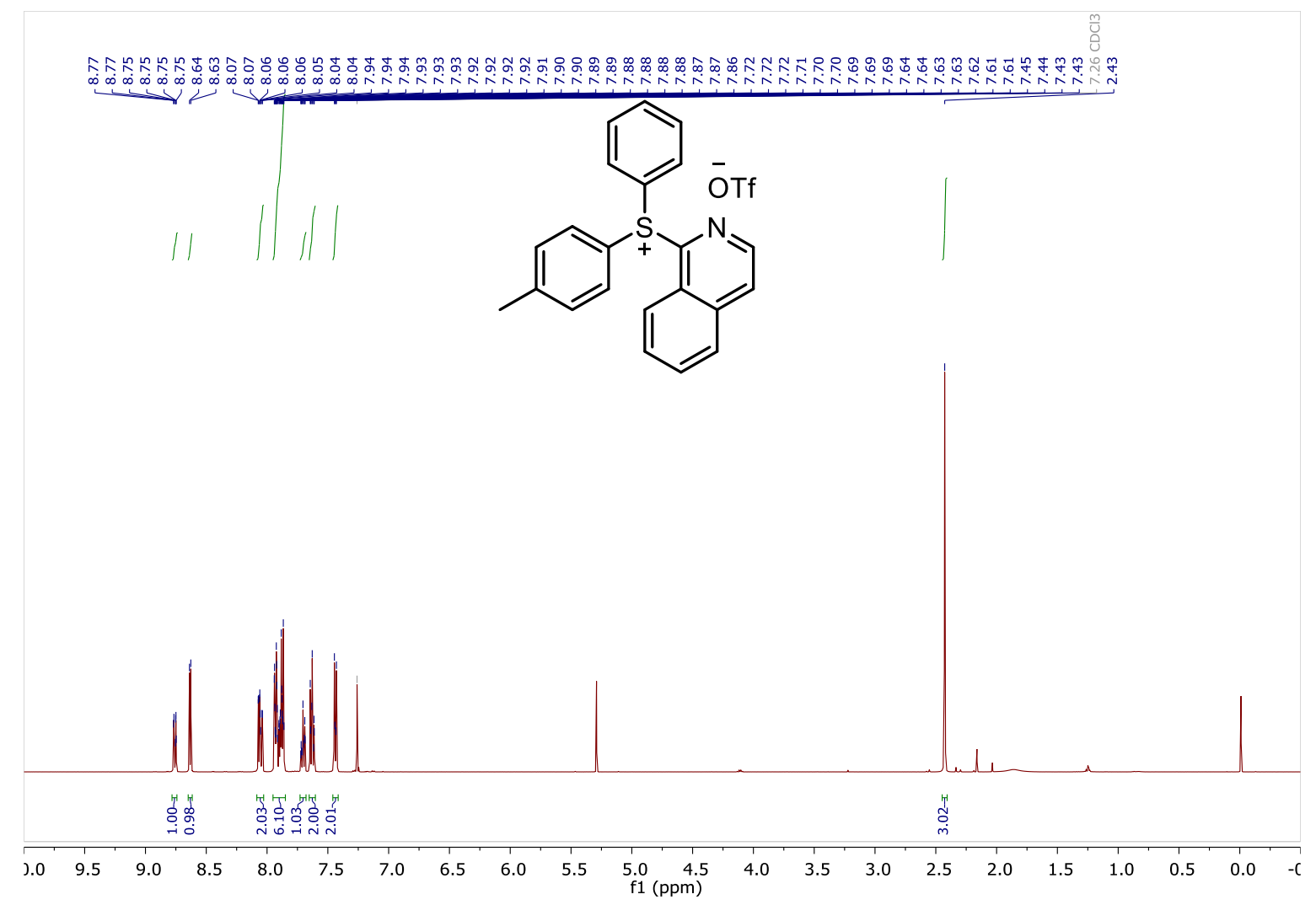

${ }^{13} \mathrm{C}$ NMR (126 MHz, $\left.\mathrm{CDCl}_{3}\right): 2 \mathrm{c}$

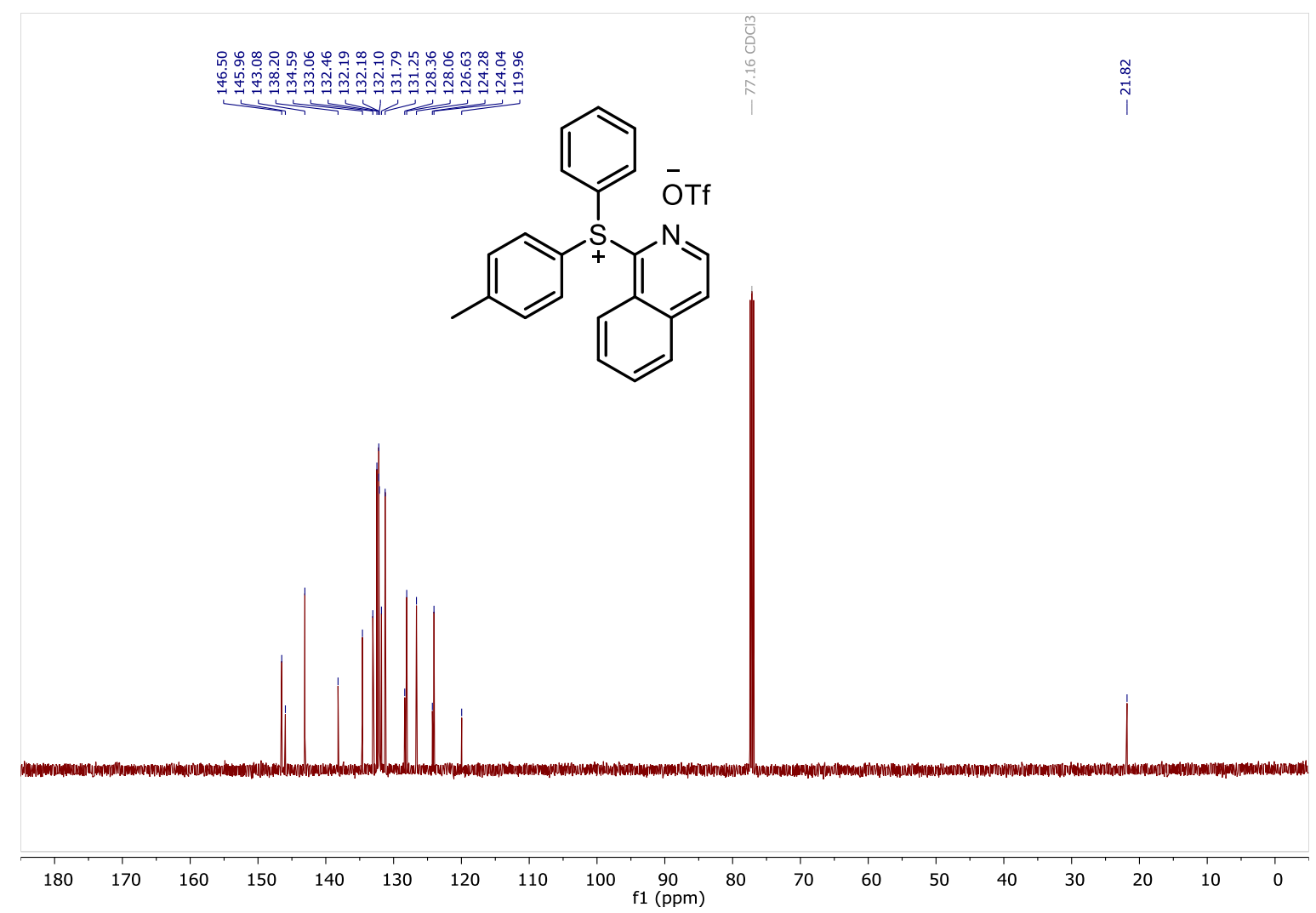


${ }^{1} \mathrm{H}$ NMR (500 MHz, $\mathrm{CDCl}_{3}$ ): $2 \mathrm{~d}$

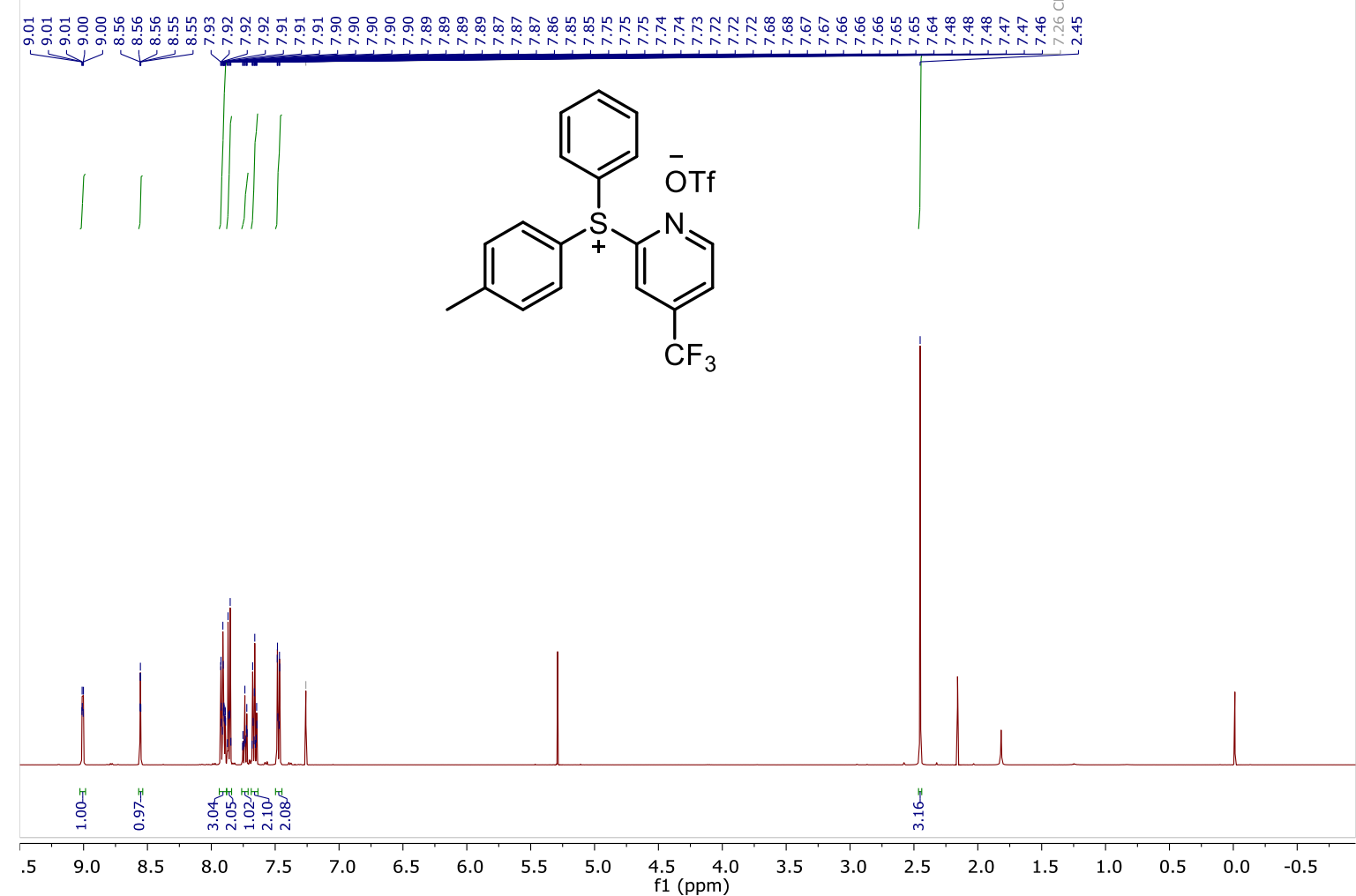

${ }^{13} \mathrm{C}$ NMR (126 MHz, $\left.\mathrm{CDCl}_{3}\right): 2 \mathrm{~d}$

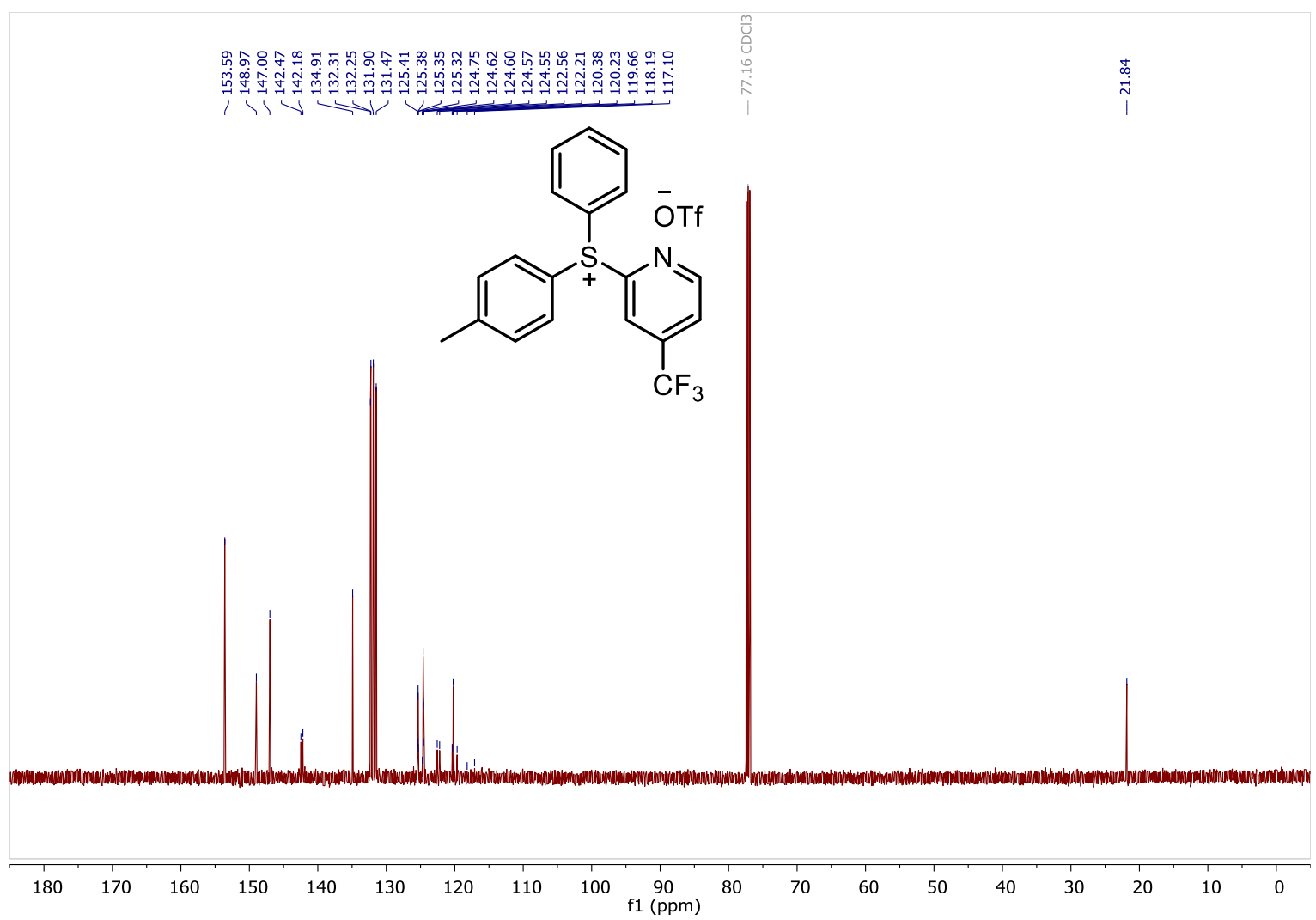


${ }^{1} \mathrm{H}$ NMR (500 MHz, $\mathrm{CDCl}_{3}$ ): $2 \mathrm{e}$

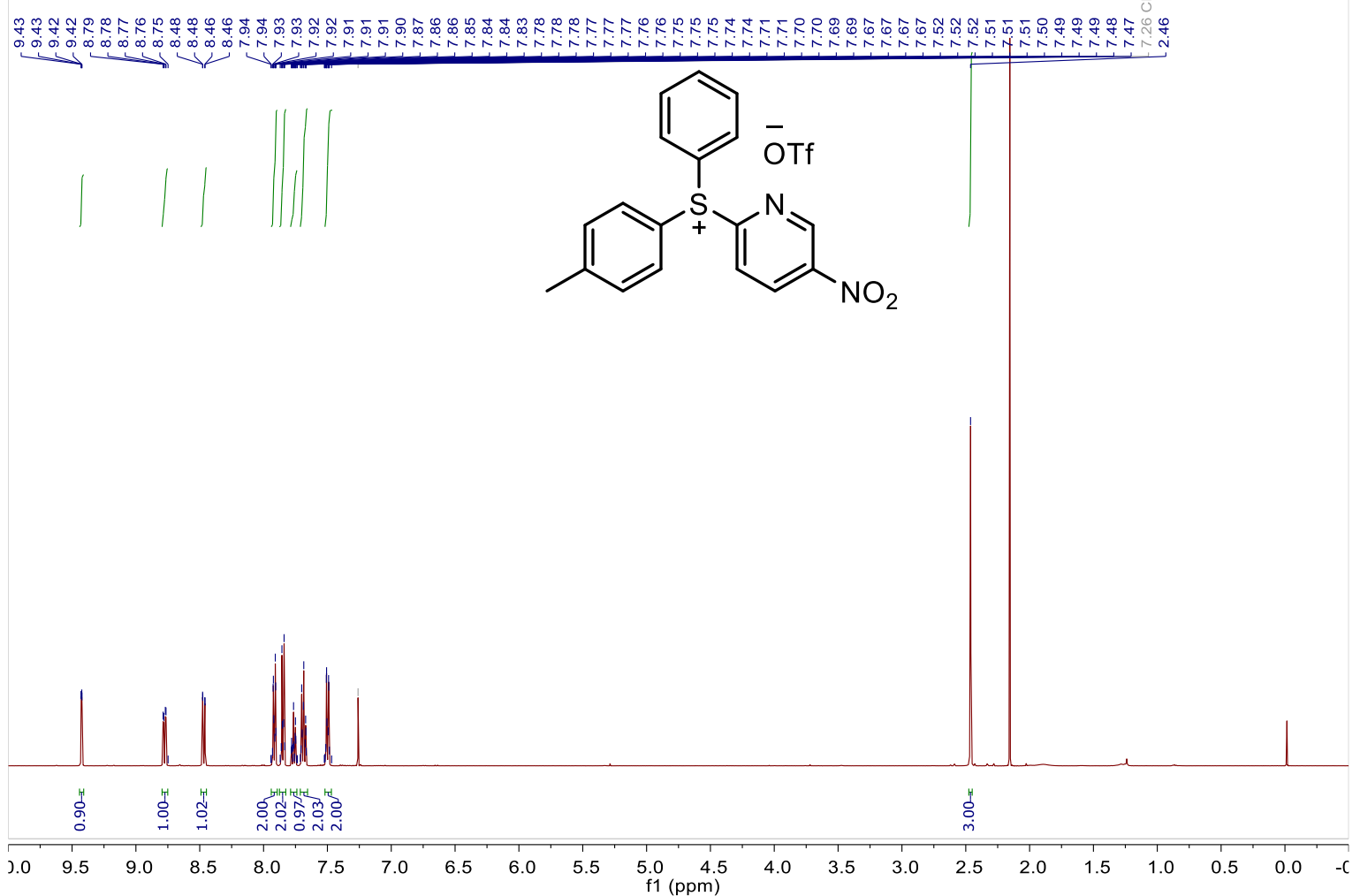

${ }^{13} \mathrm{C}$ NMR (101 MHz, $\left.\mathrm{CDCl}_{3}\right)$ : $2 \mathrm{e}$

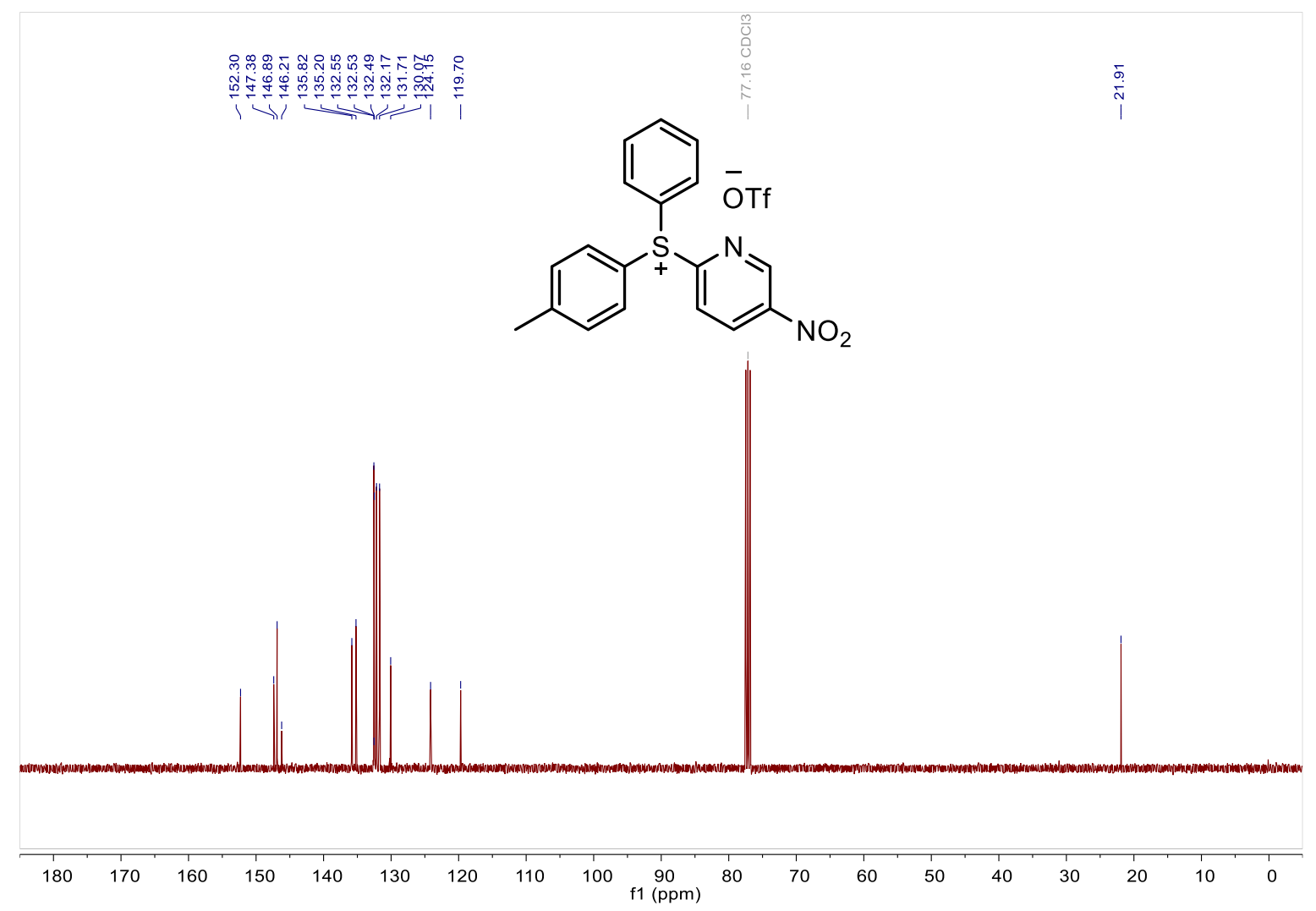


${ }^{1} \mathrm{H}$ NMR (400 MHz, $\mathrm{CDCl}_{3}$ ): $2 \mathrm{f}$

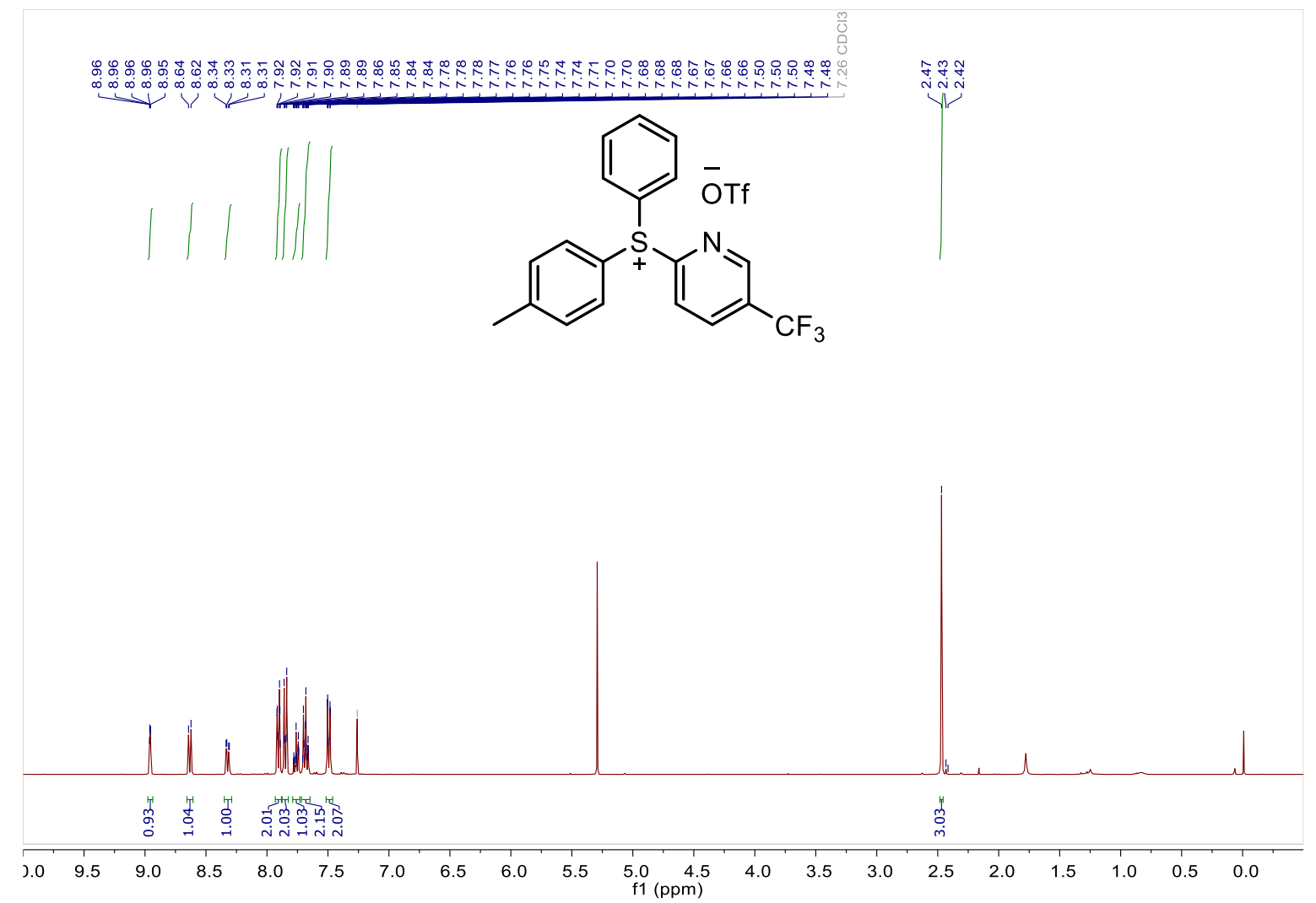

${ }^{13} \mathrm{C}$ NMR (101 MHz, $\left.\mathrm{CDCl}_{3}\right): 2 \mathrm{f}$

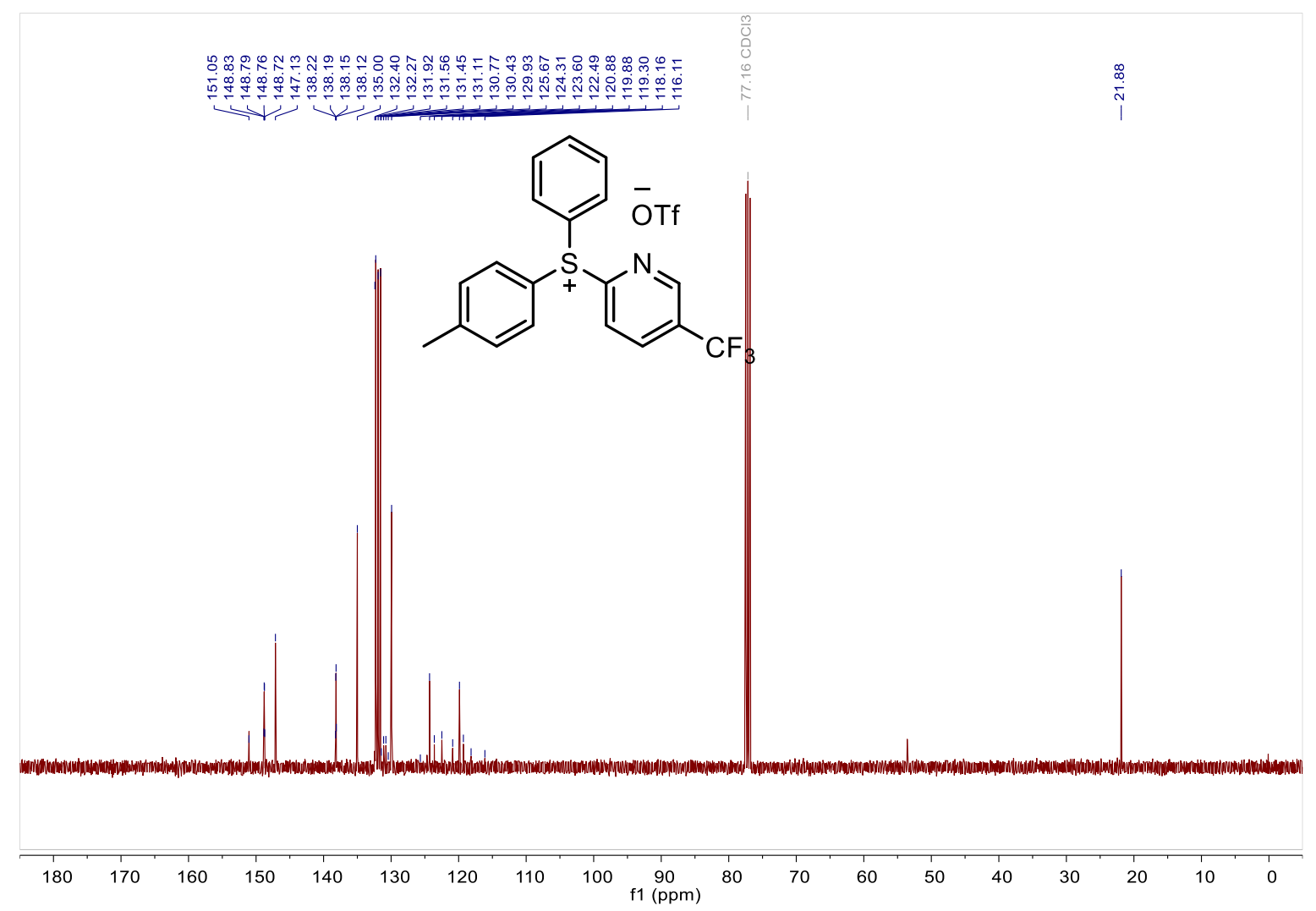


${ }^{1} \mathrm{H}$ NMR (500 MHz, $\left.\mathrm{CDCl}_{3}\right): 2 \mathrm{~g}$

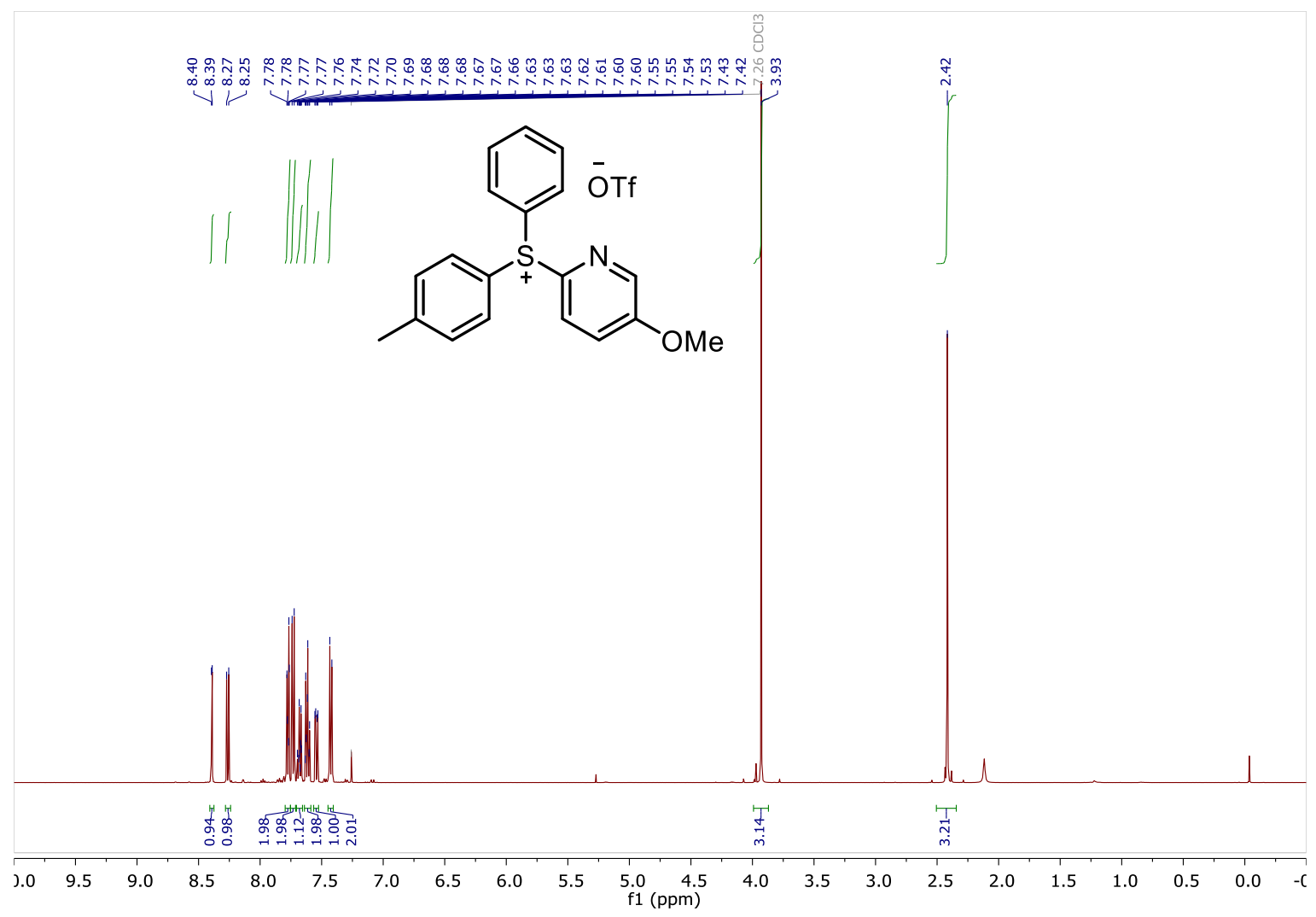

${ }^{13} \mathrm{C}$ NMR (126 MHz, $\left.\mathrm{CDCl}_{3}\right): 2 \mathrm{~g}$

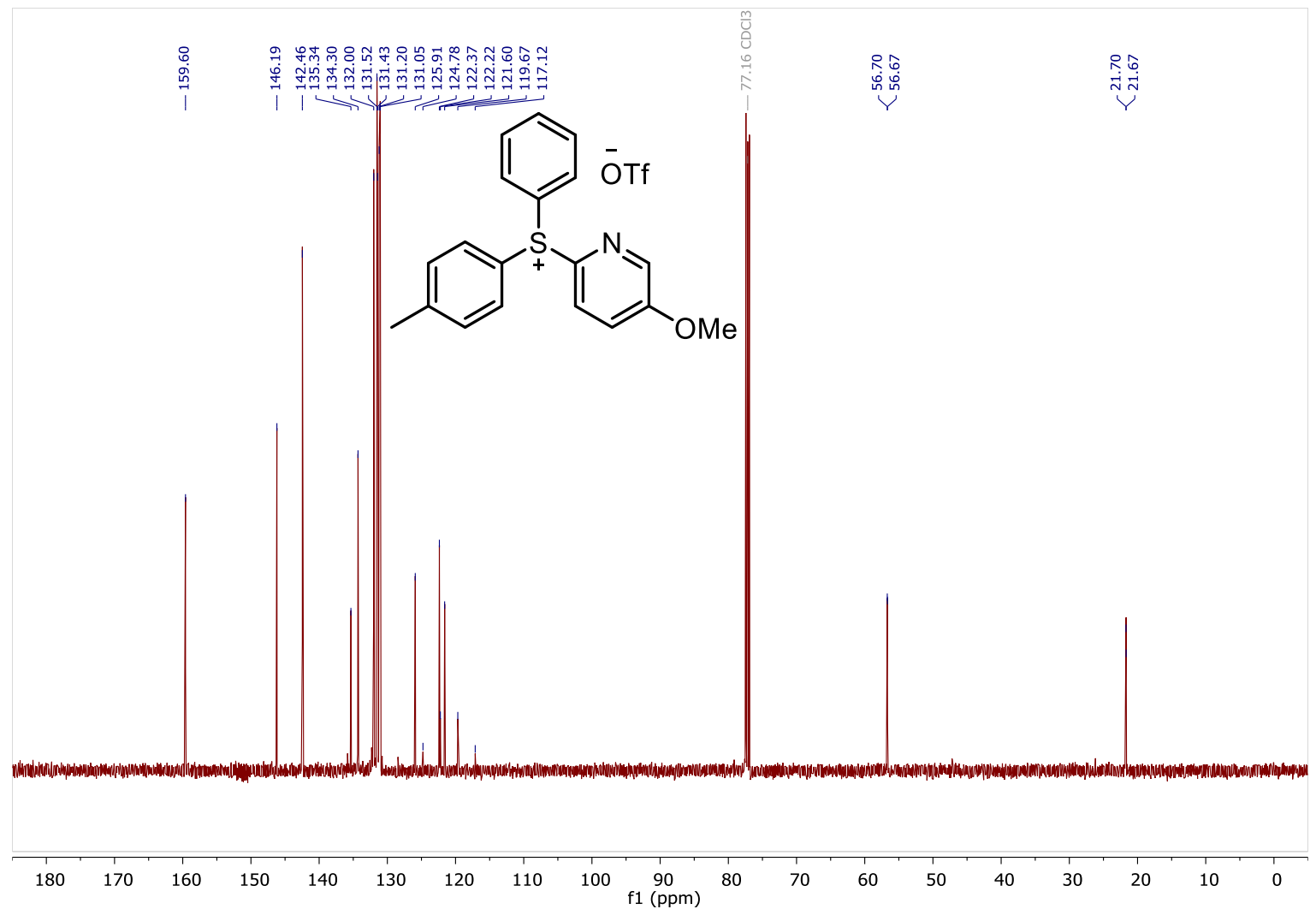

S54 
${ }^{1} \mathrm{H}$ NMR (500 MHz, $\mathrm{CDCl}_{3}$ ): $2 \mathrm{~h}$

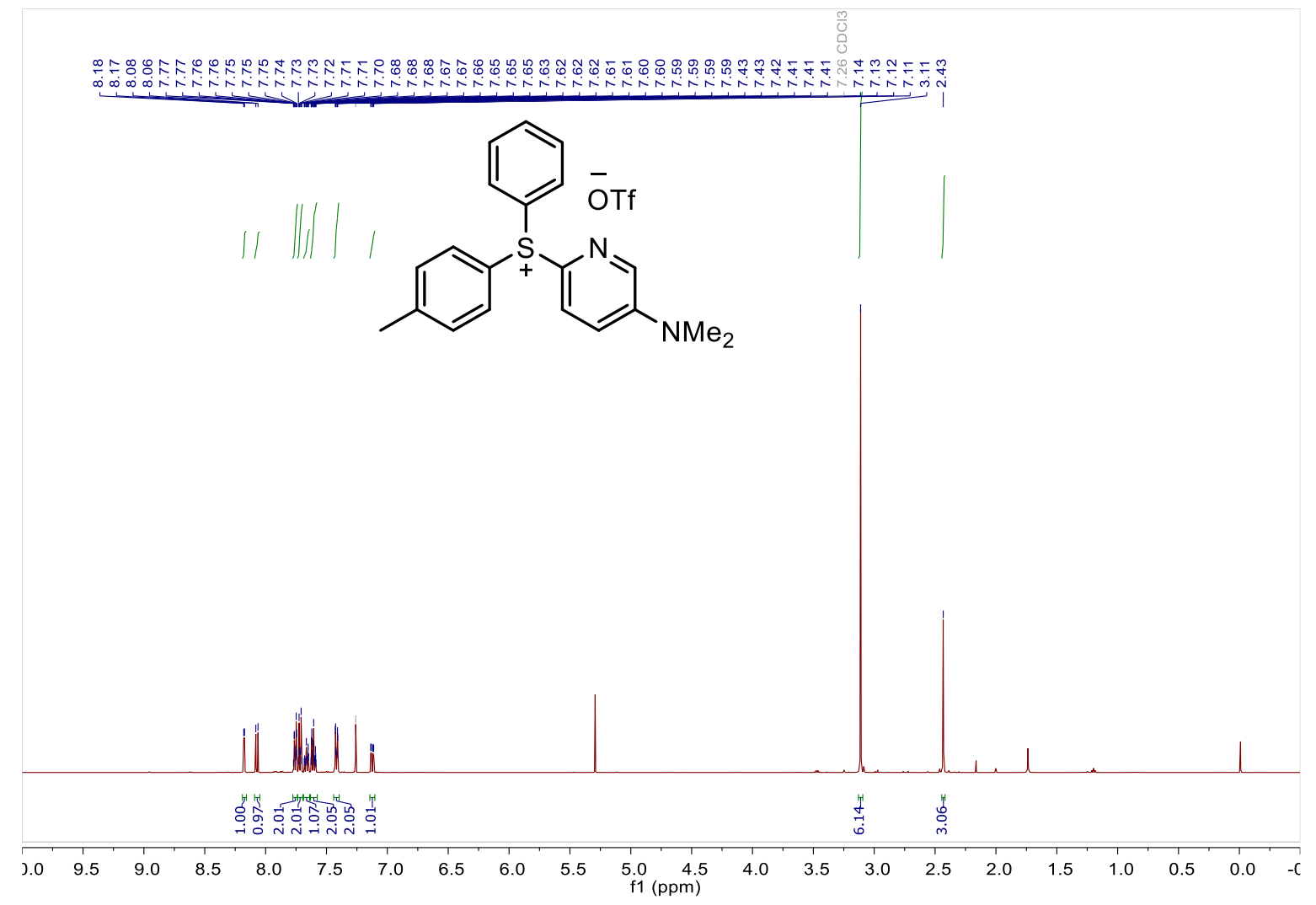

${ }^{13} \mathrm{C}$ NMR (126 MHz, $\left.\mathrm{CDCl}_{3}\right): 2 \mathrm{~h}$
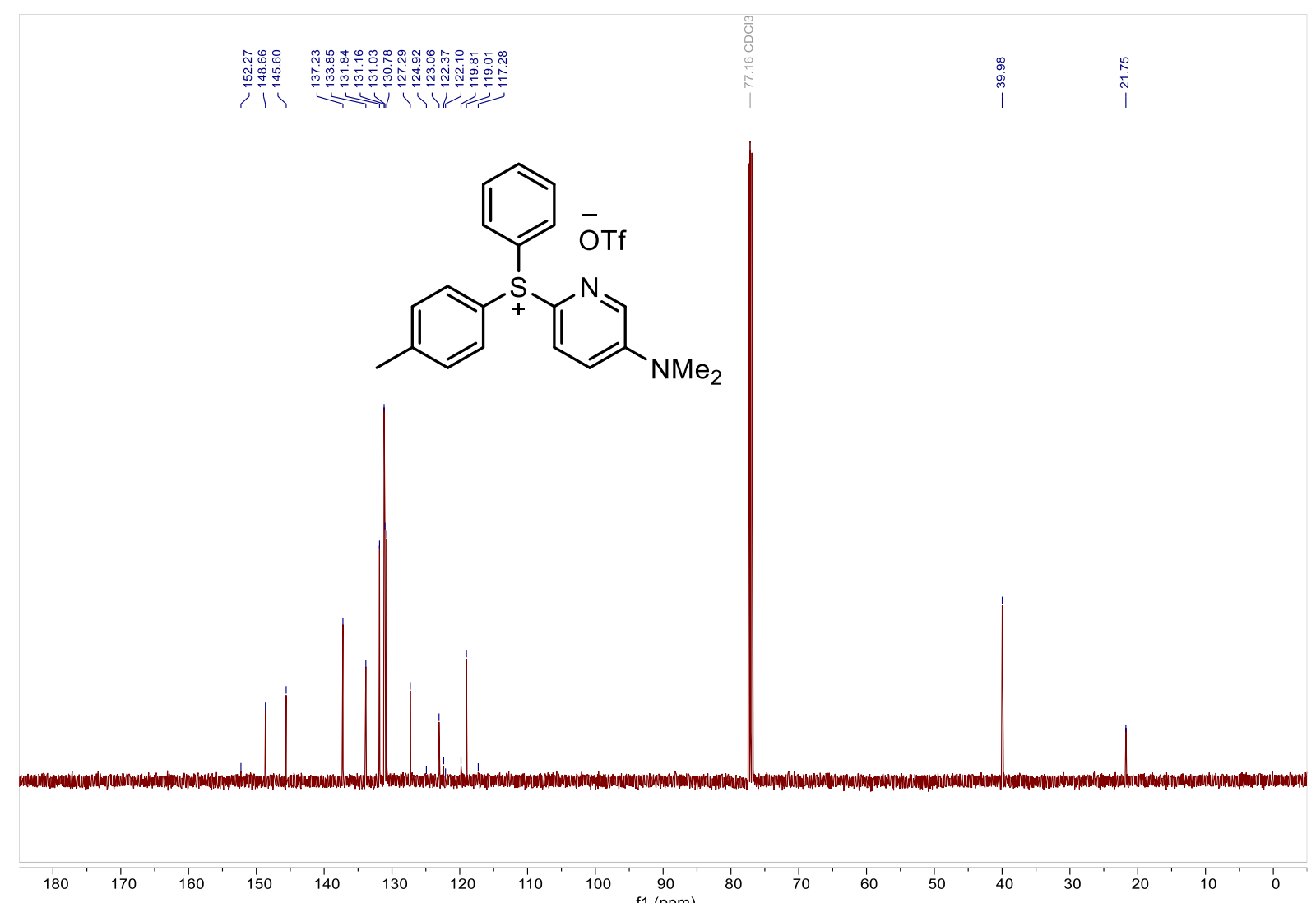
${ }^{1} \mathrm{H}$ NMR (400 MHz, $\mathrm{CDCl}_{3}$ ): 2i

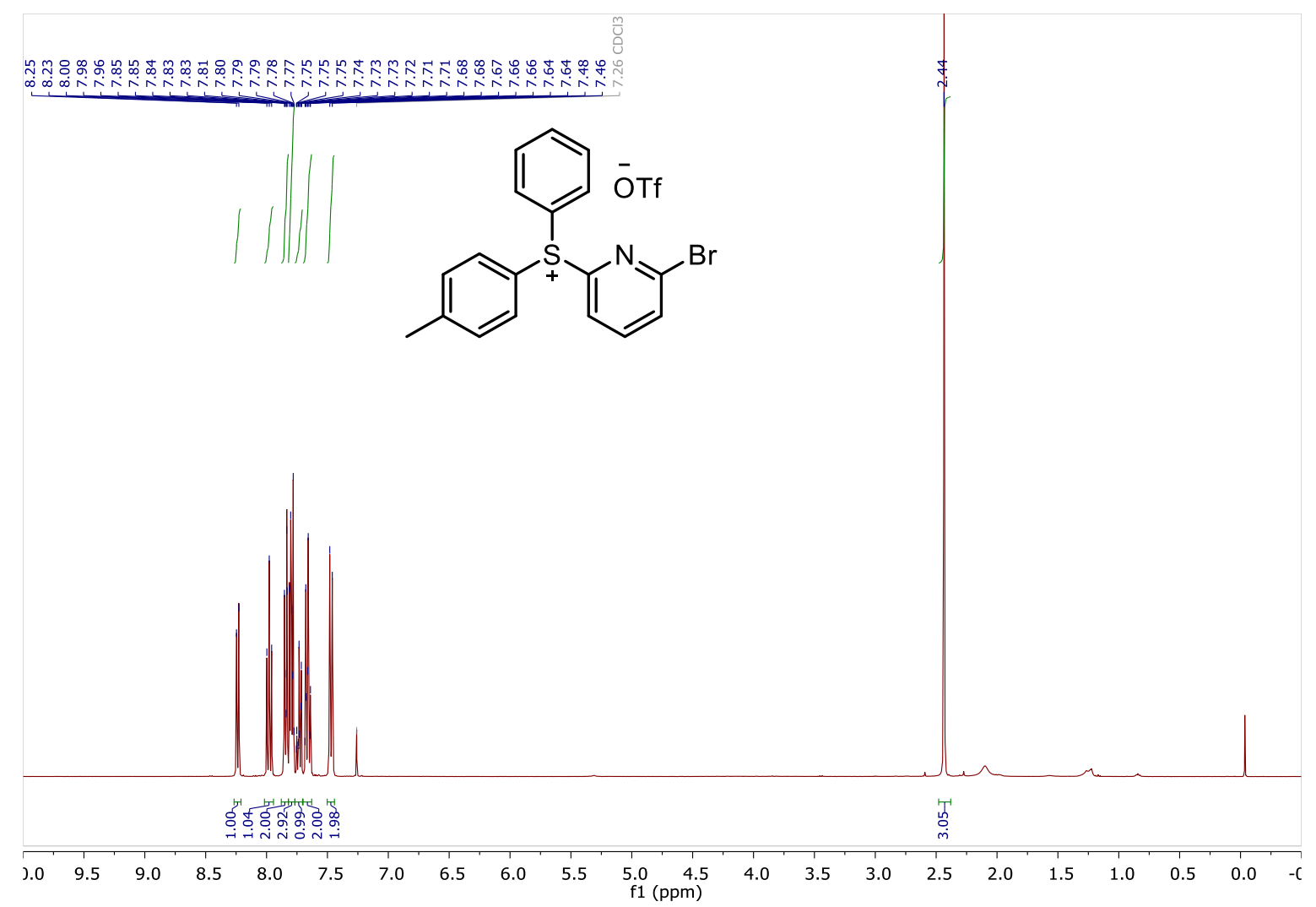

${ }^{13} \mathrm{C}$ NMR (126 MHz, $\left.\mathrm{CDCl}_{3}\right): 2 \mathrm{i}$

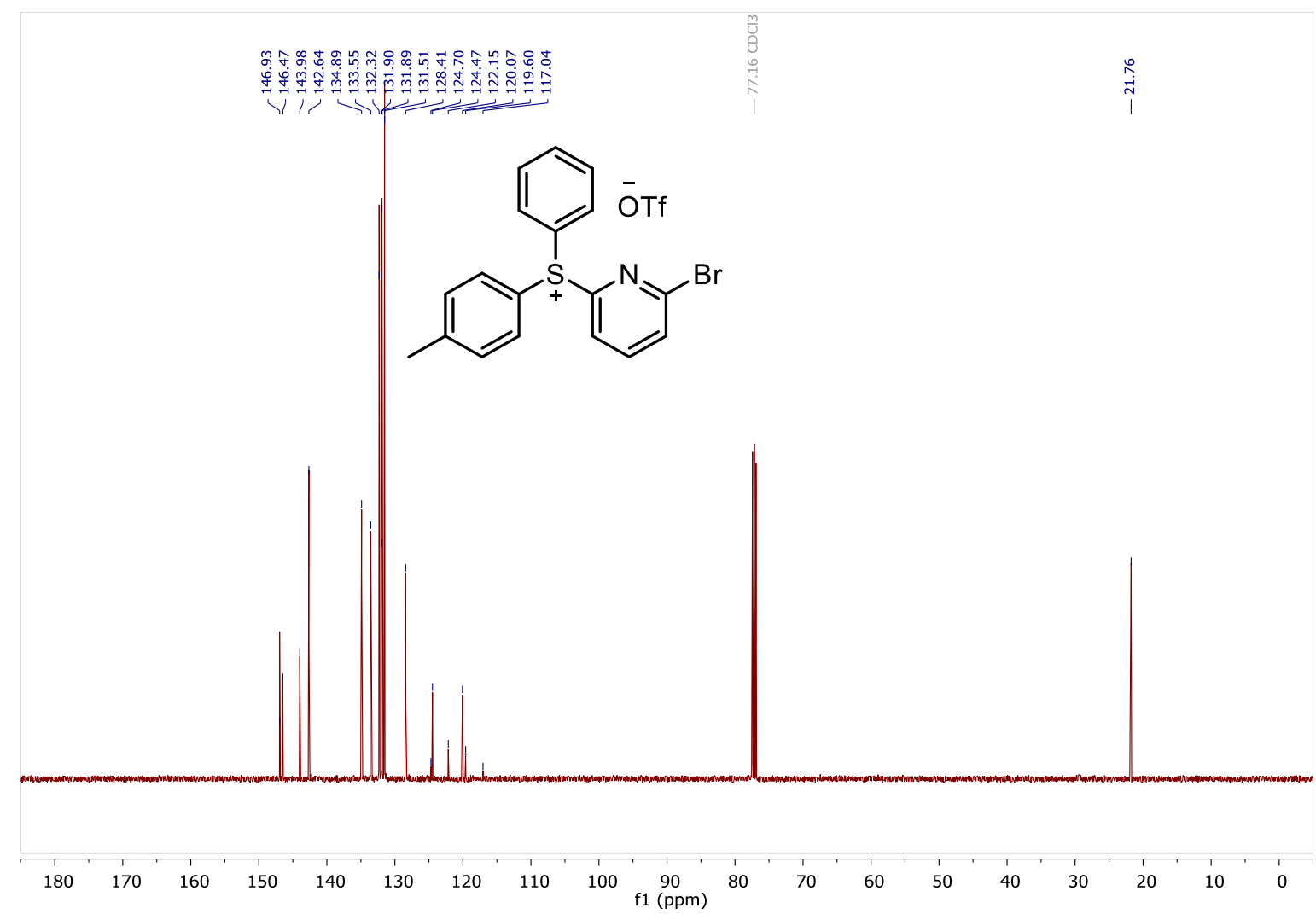


${ }^{1} \mathrm{H}$ NMR (400 MHz, $\left.\mathrm{CDCl}_{3}\right): 3$

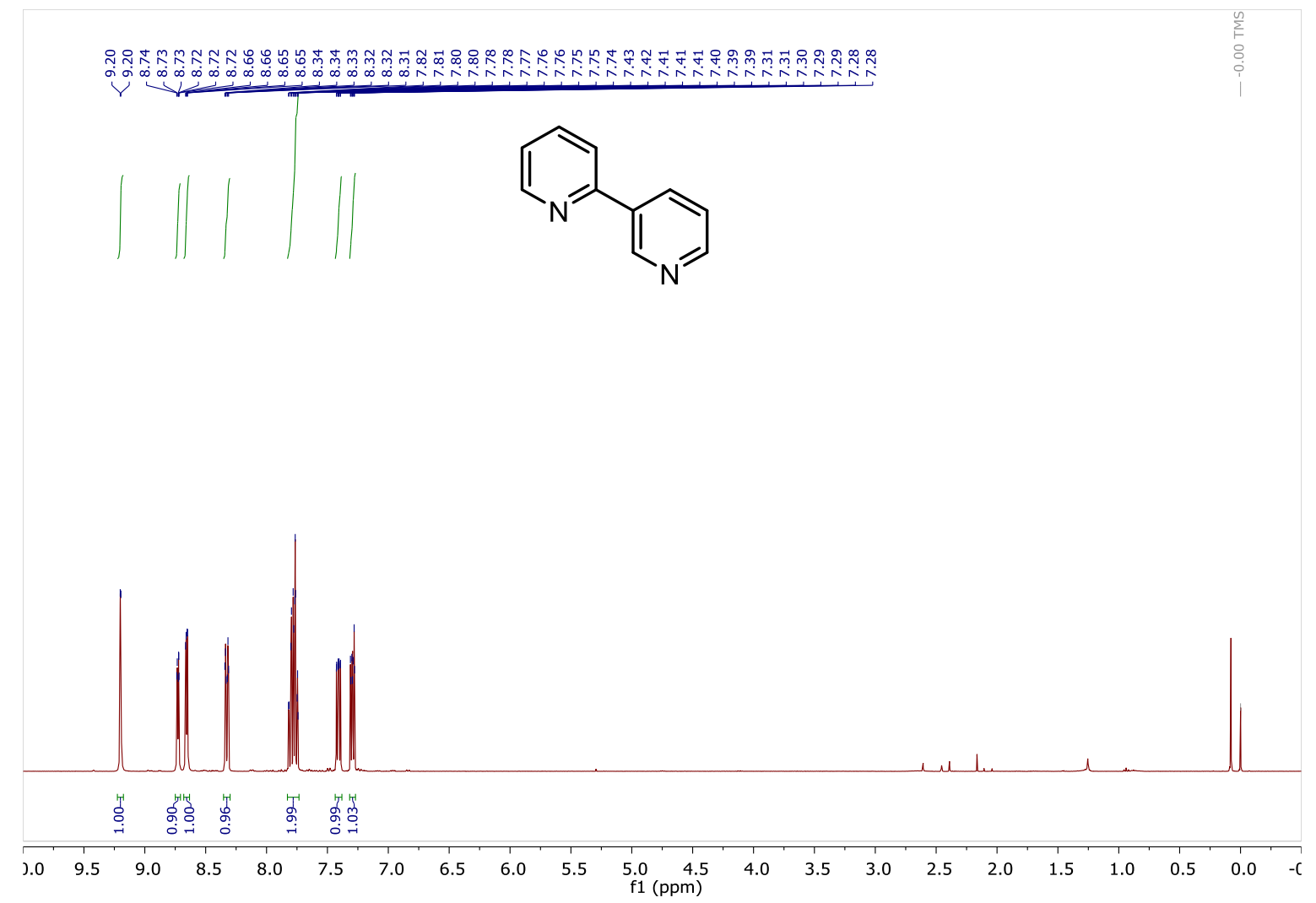

${ }^{13} \mathrm{C}$ NMR (101 MHz, $\left.\mathrm{CDCl}_{3}\right): 3$

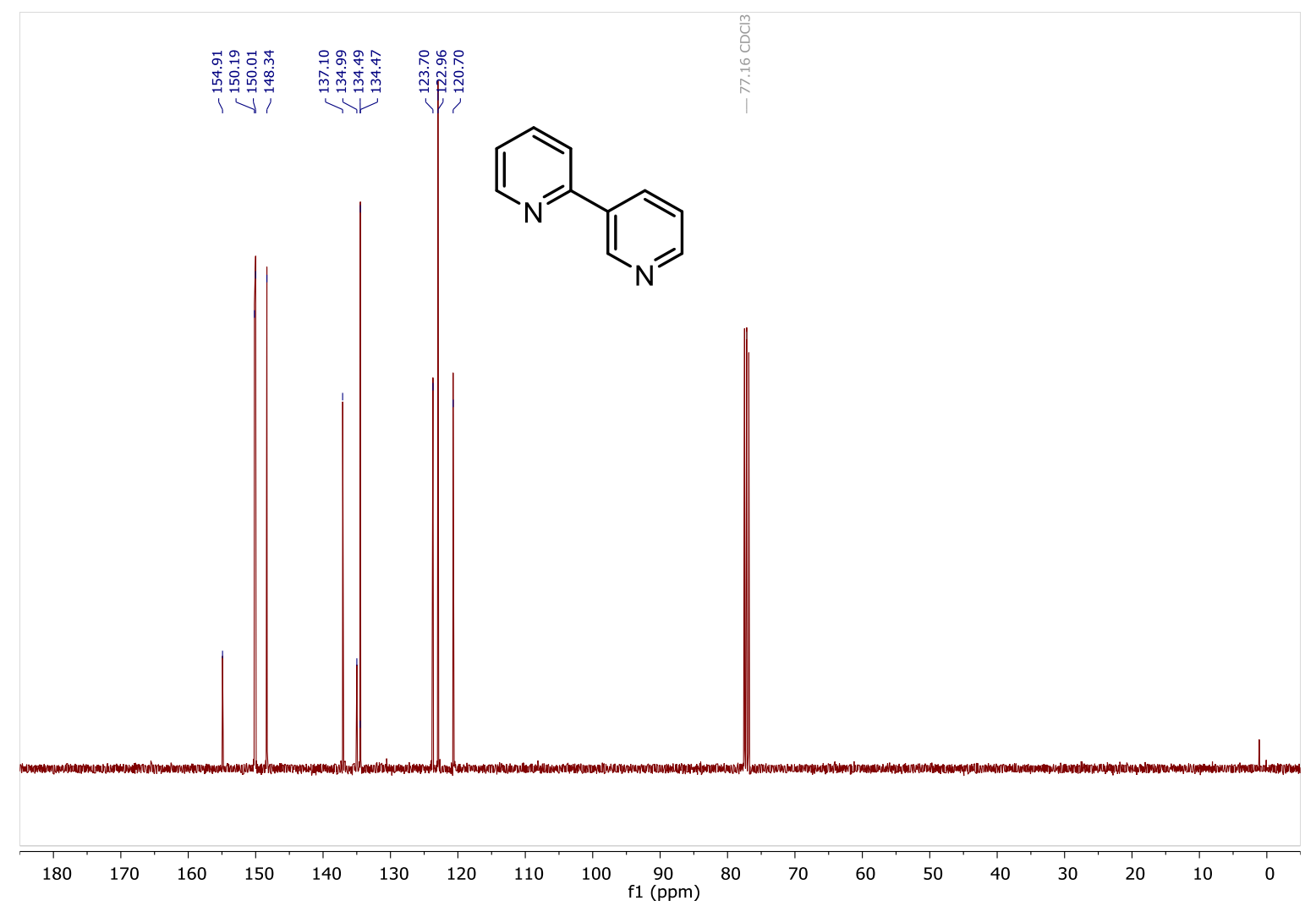


${ }^{1} \mathrm{H}$ NMR (400 MHz, $\mathrm{CDCl}_{3}$ ): 4

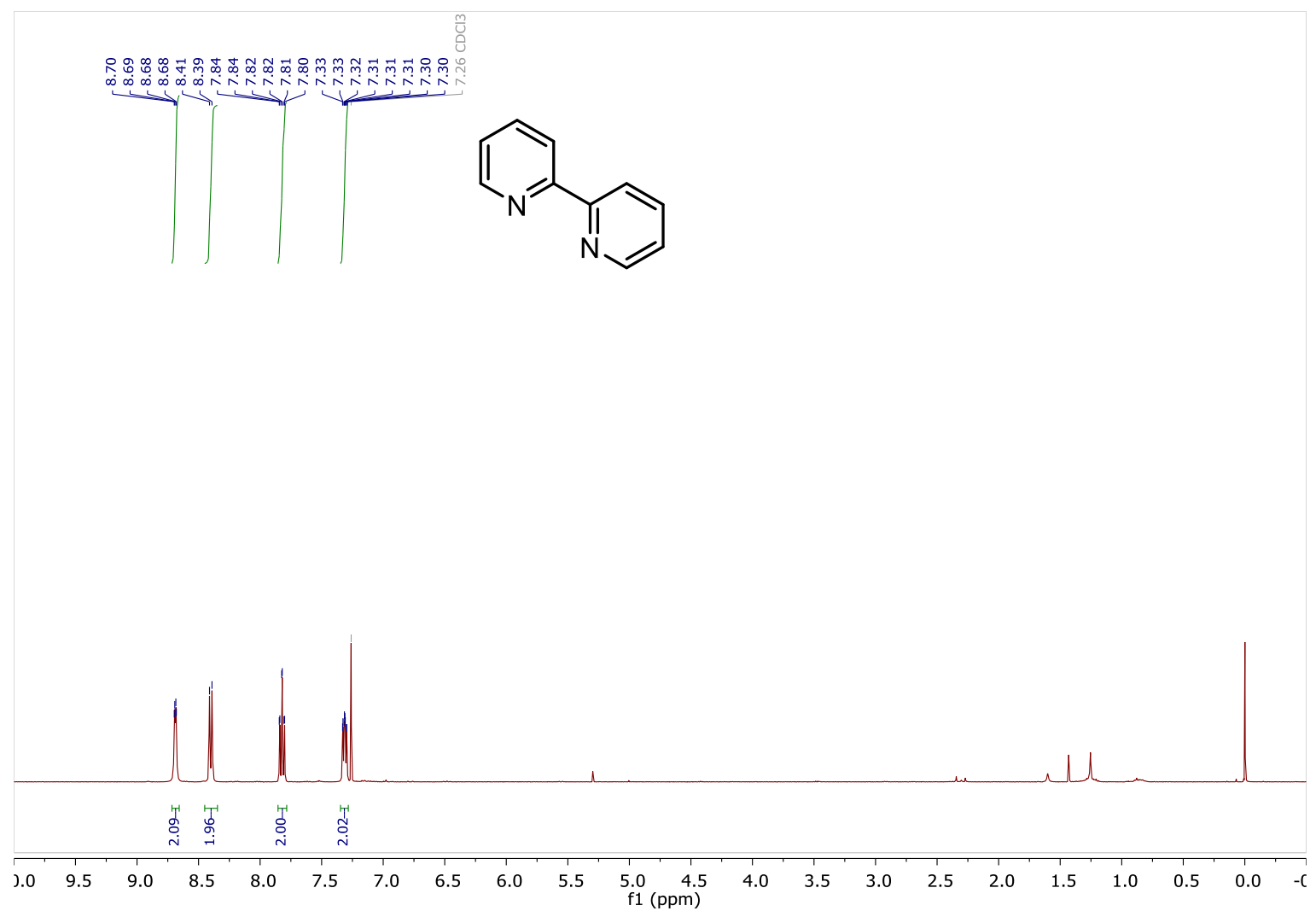

${ }^{13} \mathrm{C}$ NMR (101 MHz, $\left.\mathrm{CDCl}_{3}\right): 4$

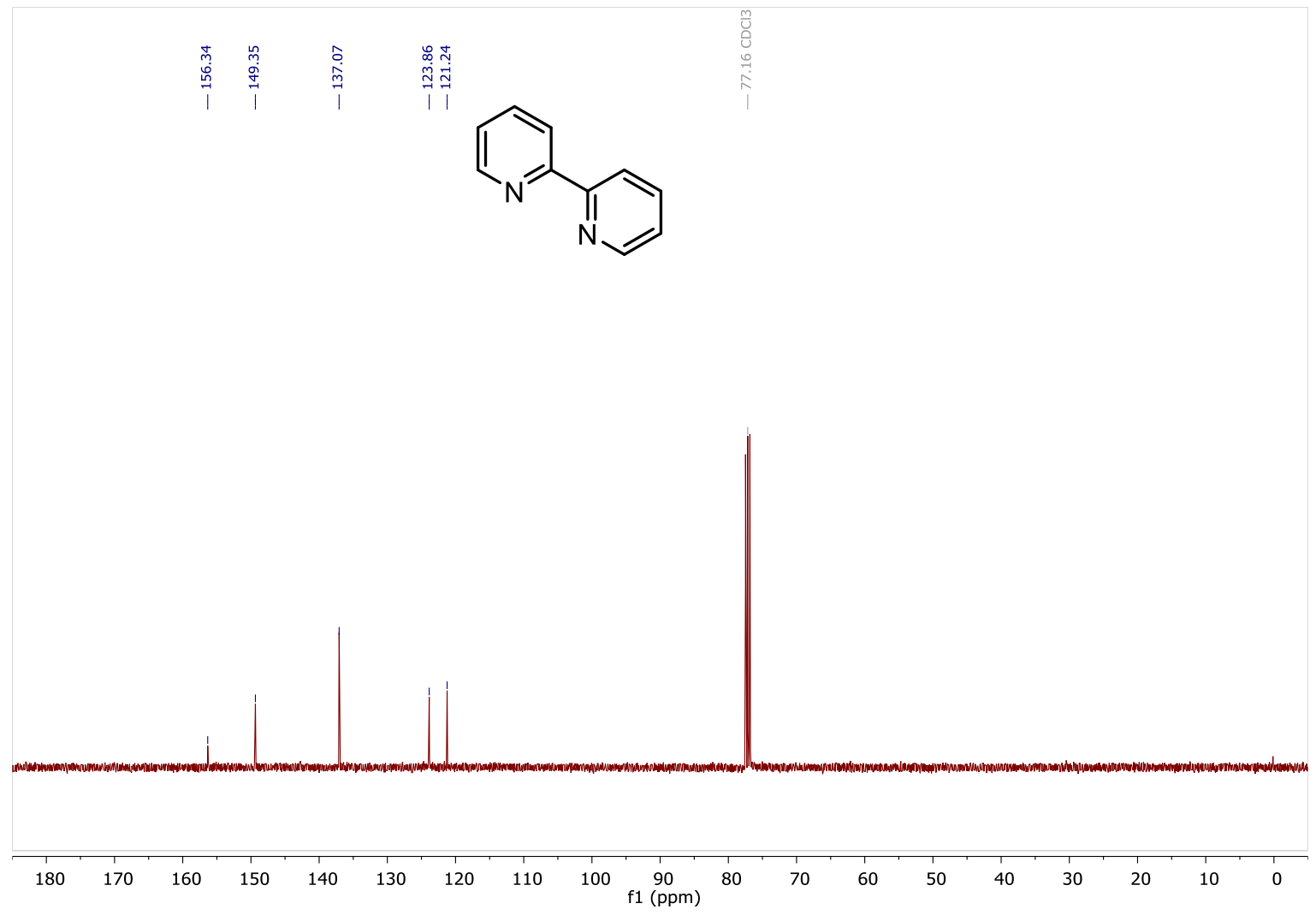

S58 
${ }^{1} \mathrm{H}$ NMR (500 MHz, $\left.\mathrm{CDCl}_{3}\right): 5$

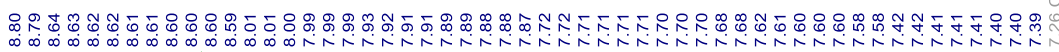<smiles>c1ccc(-c2nccc3ccccc23)nc1</smiles>

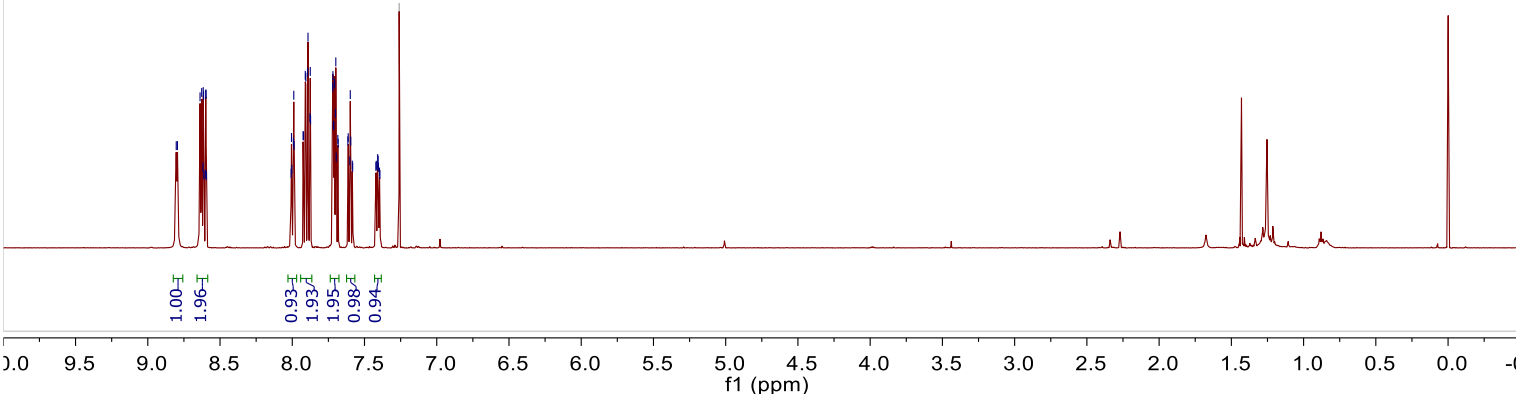

${ }^{13} \mathrm{C}$ NMR (101 MHz, $\left.\mathrm{CDCl}_{3}\right): 5$

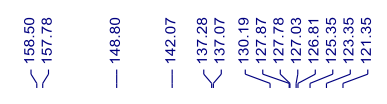<smiles>c1ccc(-c2nccc3ccccc23)nc1</smiles>
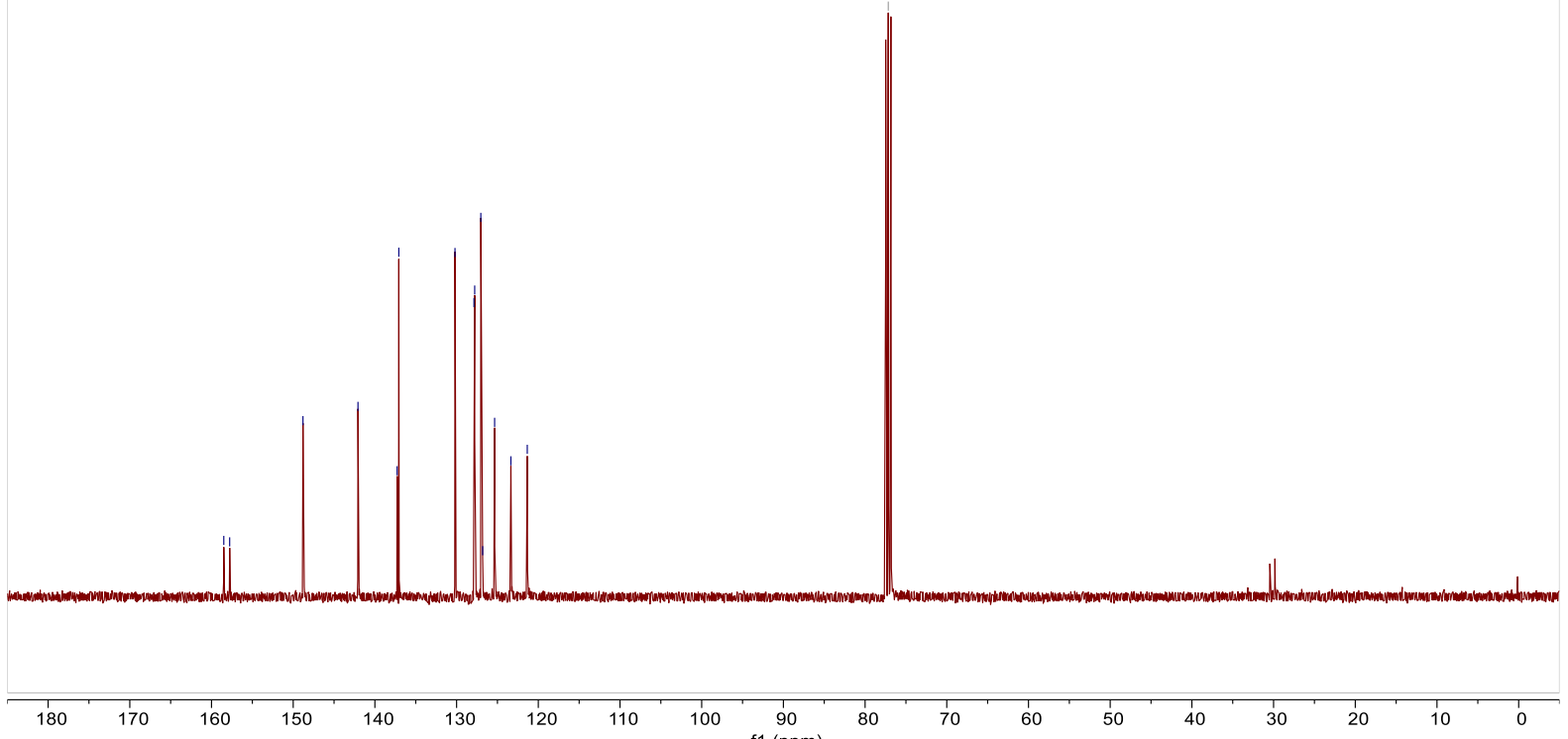
${ }^{1} \mathrm{H}$ NMR (500 MHz, $\left.\mathrm{CDCl}_{3}\right): 6$

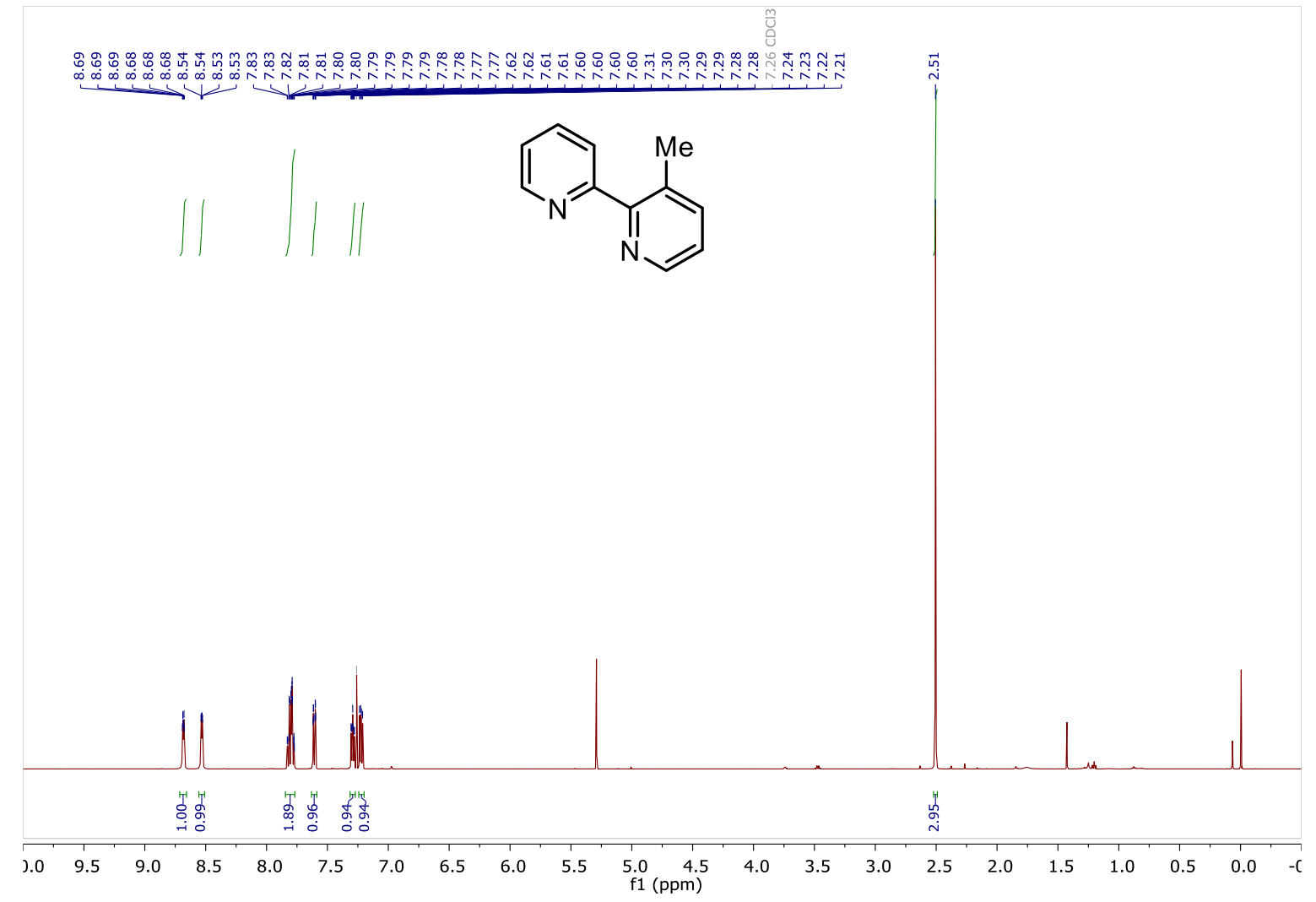

${ }^{13} \mathrm{C}$ NMR (126 MHz, $\left.\mathrm{CDCl}_{3}\right): 6$

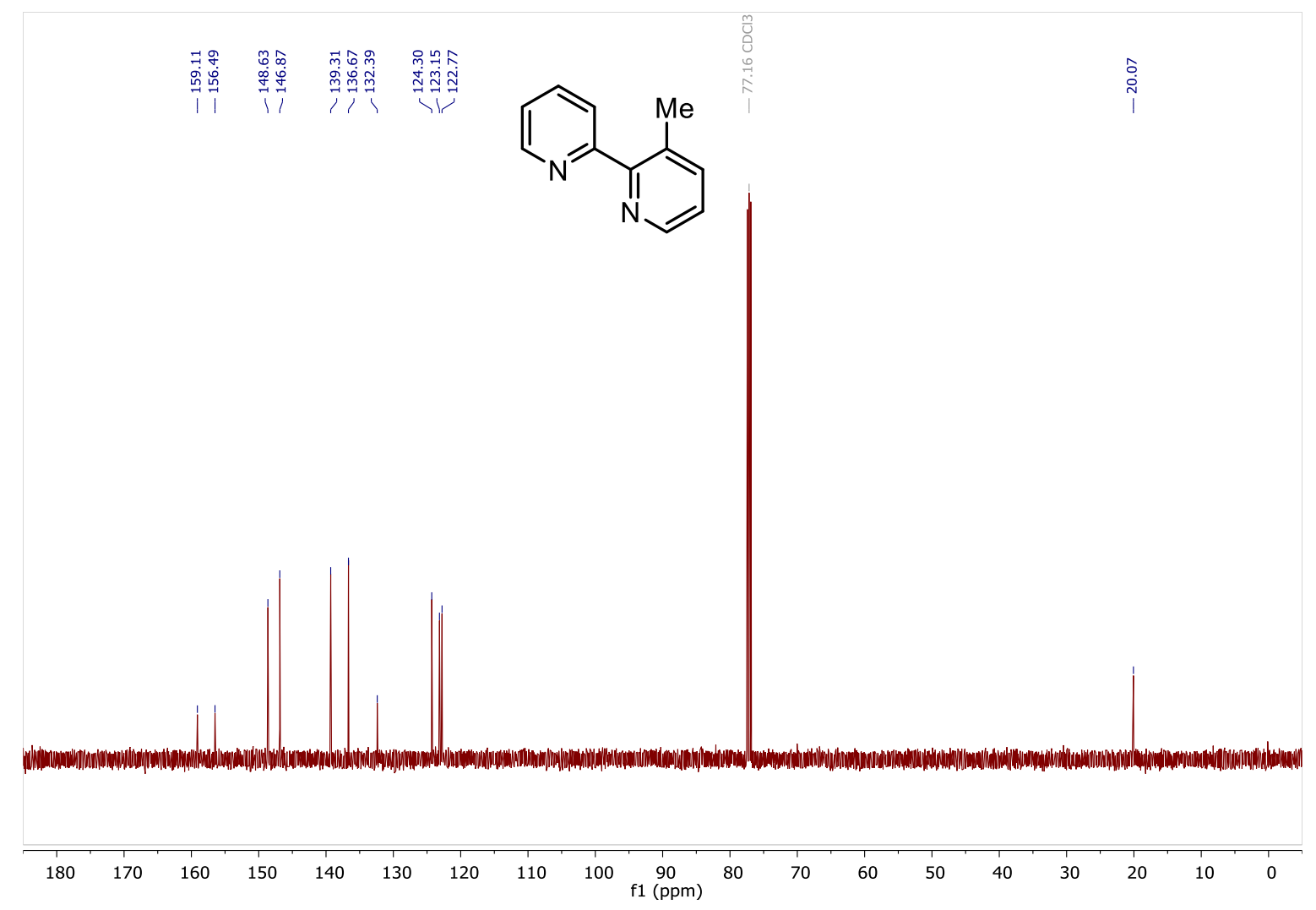


${ }^{1} \mathrm{H}$ NMR (500 MHz, $\mathrm{CDCl}_{3}$ ): 7

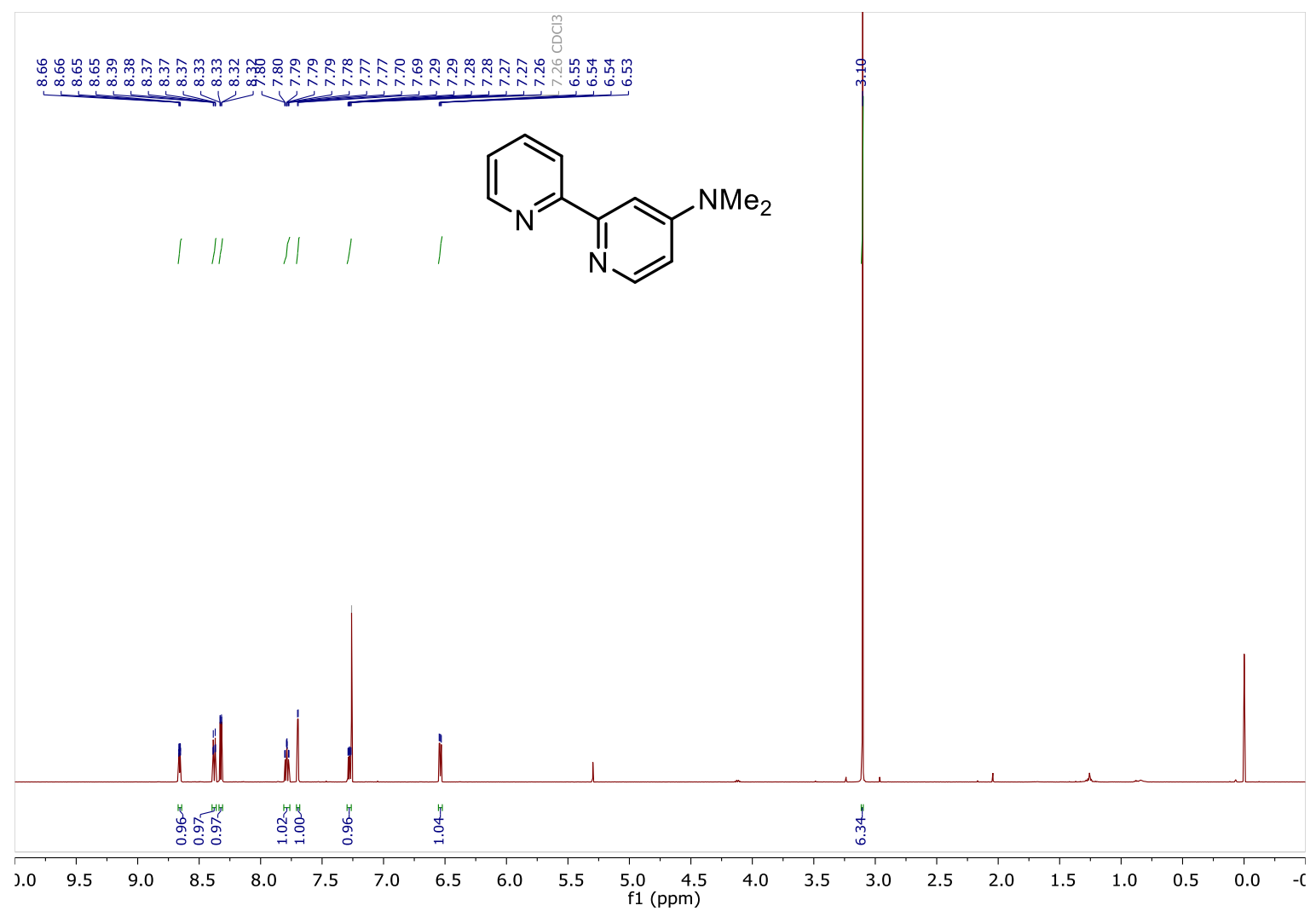

${ }^{13} \mathrm{C}$ NMR (126 MHz, $\left.\mathrm{CDCl}_{3}\right): 7$

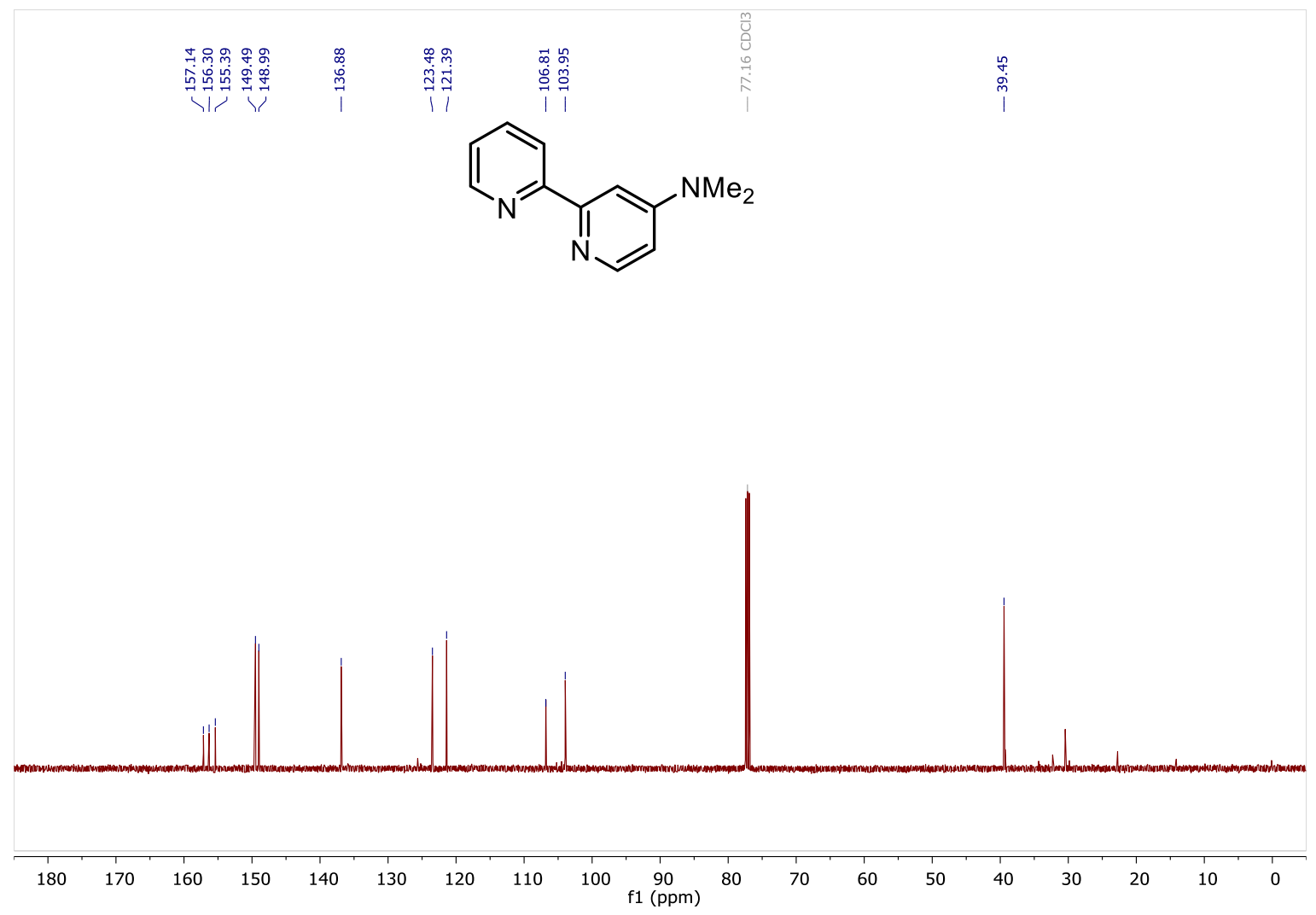

S61 
${ }^{1} \mathrm{H}$ NMR (500 MHz, $\mathrm{CDCl}_{3}$ ): 8

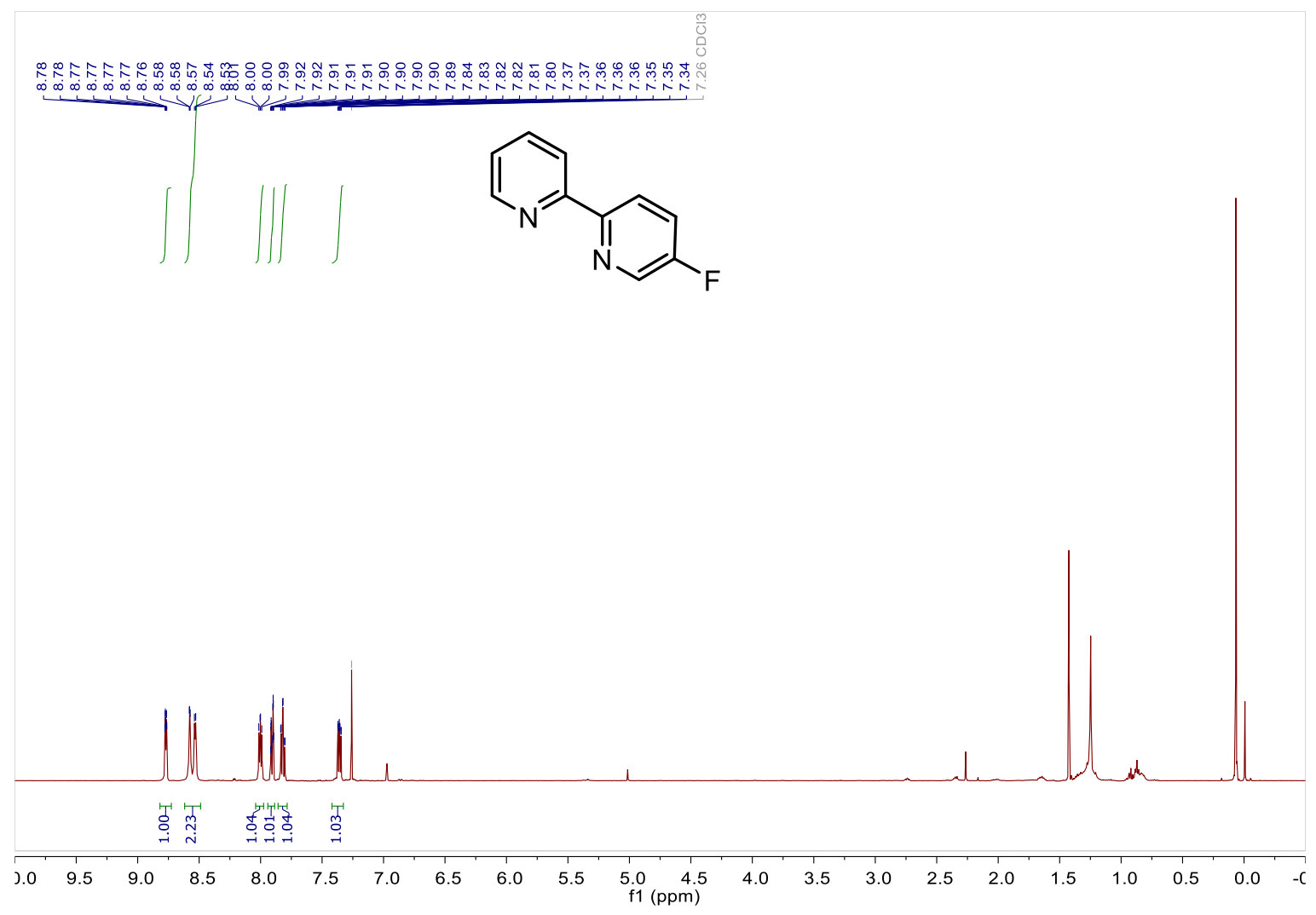

${ }^{13} \mathrm{C}$ NMR (126 MHz, $\left.\mathrm{CDCl}_{3}\right): 8$

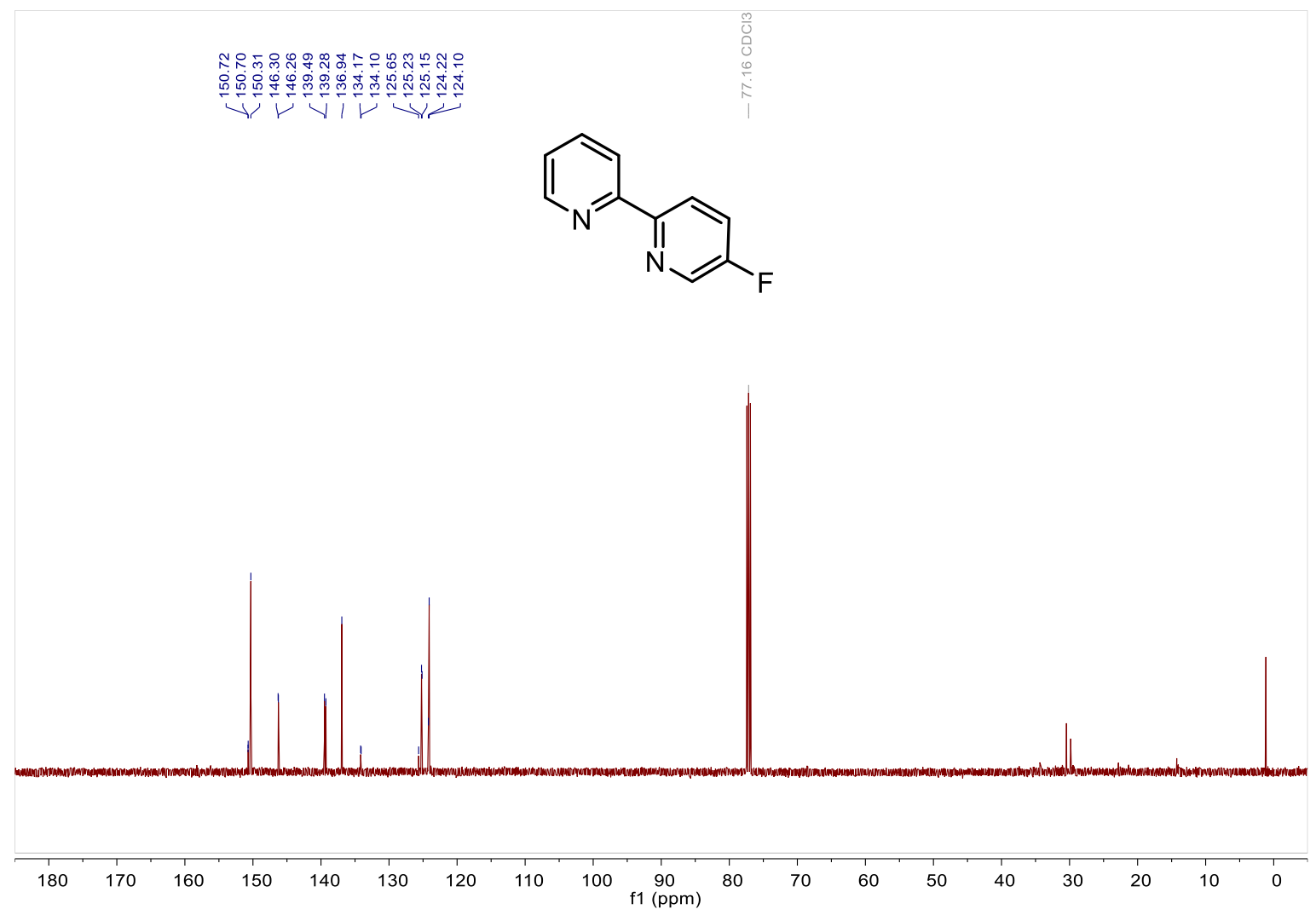

S62 
${ }^{1} \mathrm{H}$ NMR (500 MHz, $\mathrm{CDCl}_{3}$ ): 9

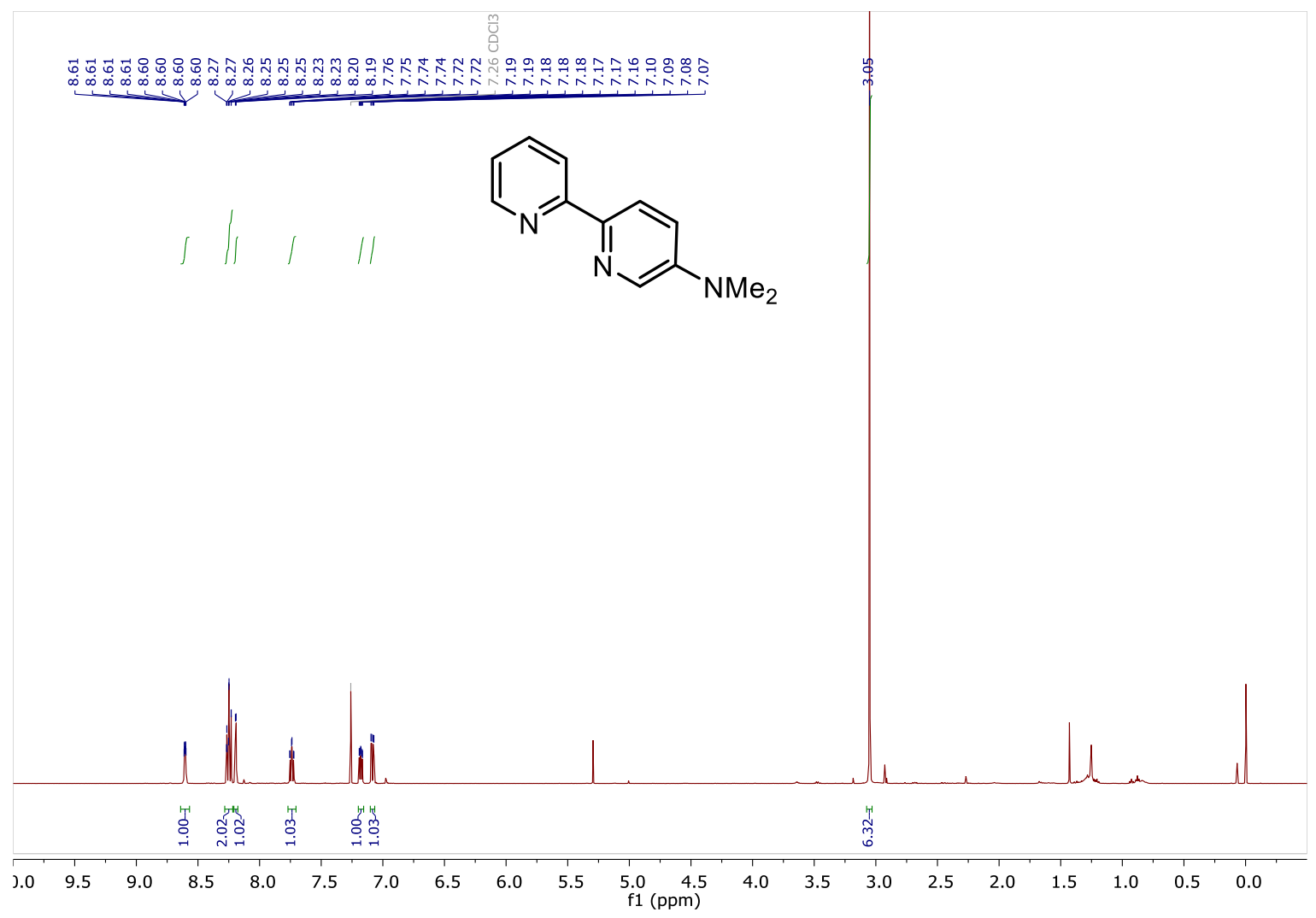

${ }^{13} \mathrm{C}$ NMR (126 MHz, $\left.\mathrm{CDCl}_{3}\right): 9$

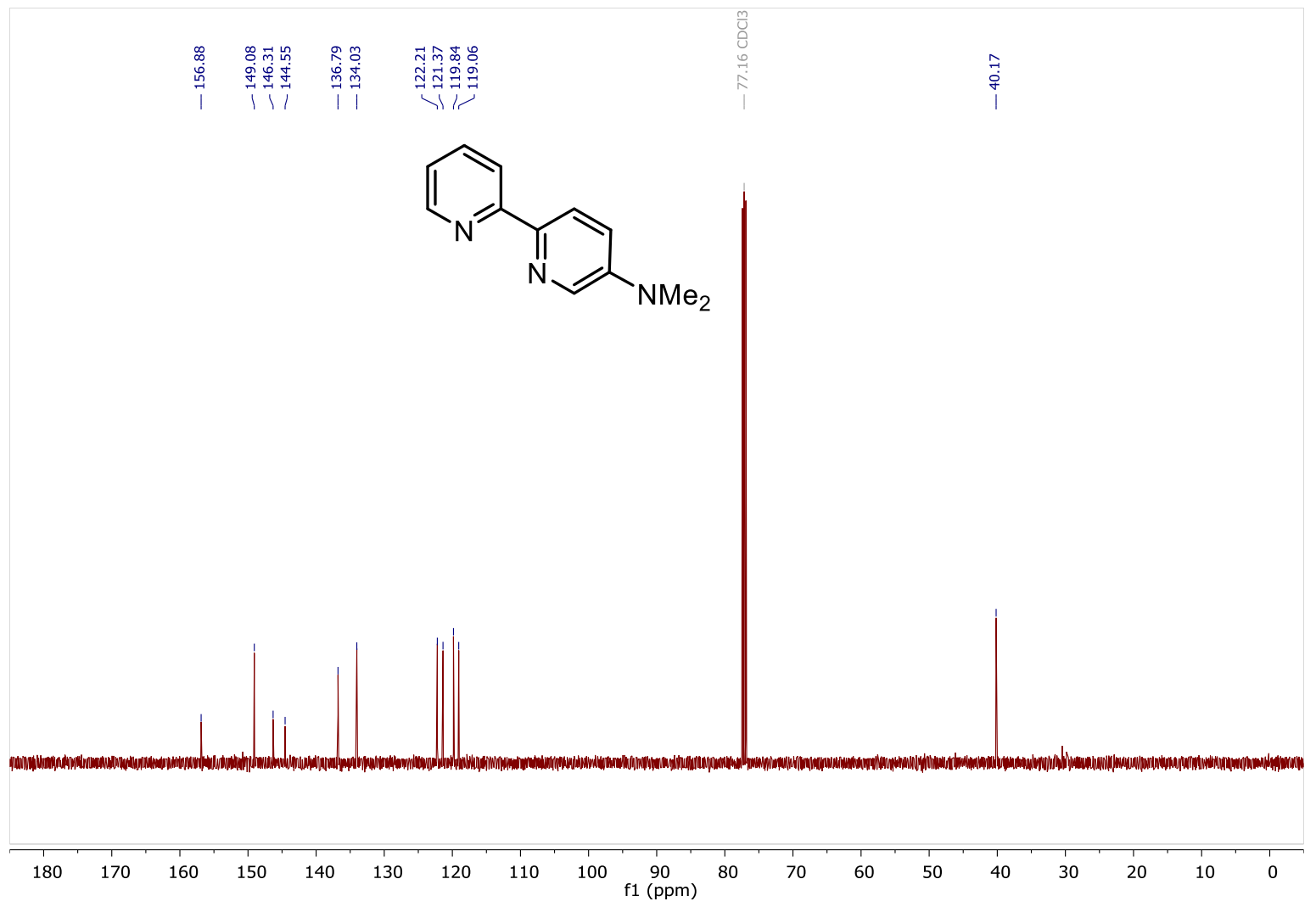

S63 
${ }^{1} \mathrm{H}$ NMR (400 MHz, $\mathrm{CDCl}_{3}$ ): 10

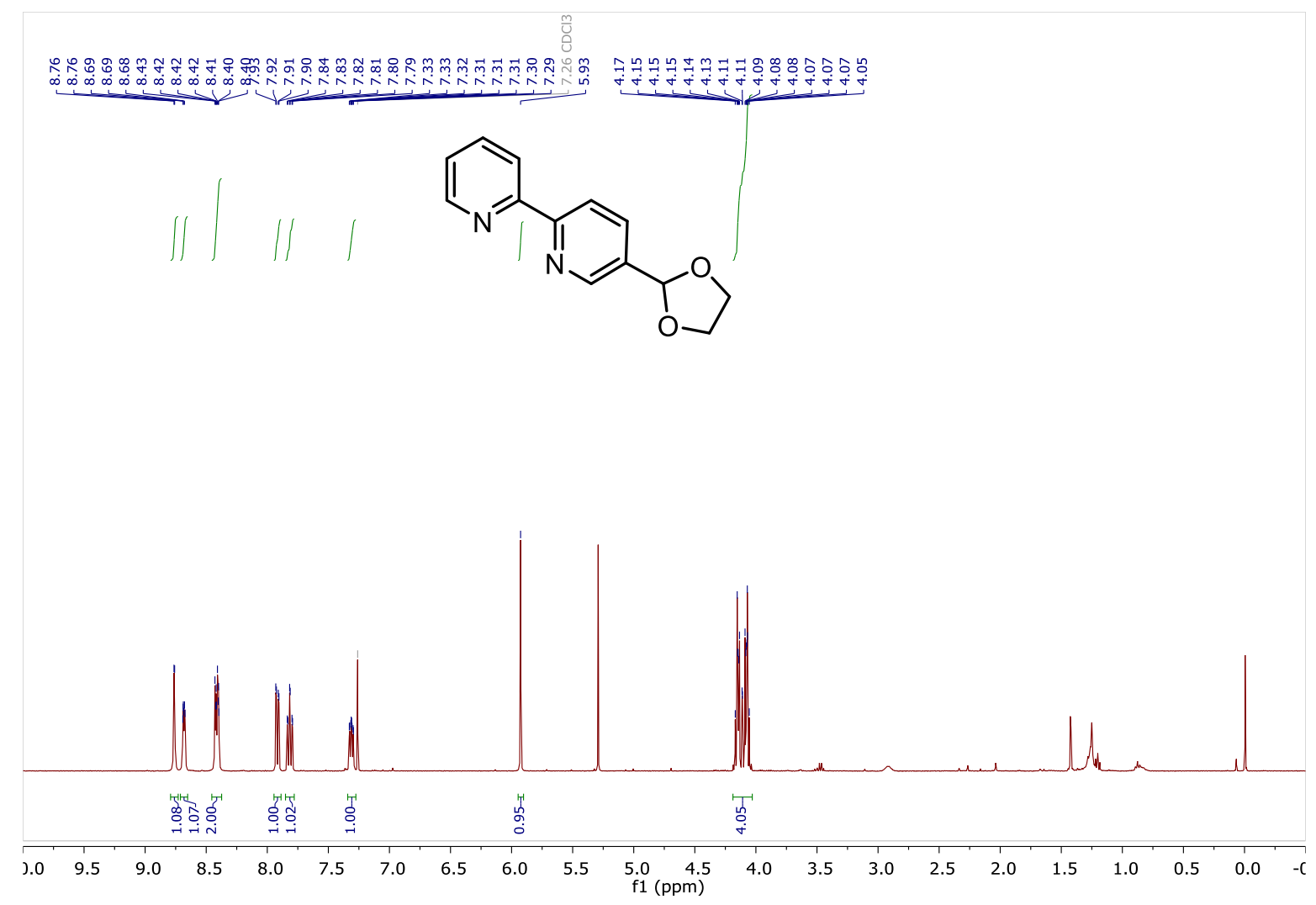

${ }^{13} \mathrm{C}$ NMR (101 MHz, $\left.\mathrm{CDCl}_{3}\right): 10$

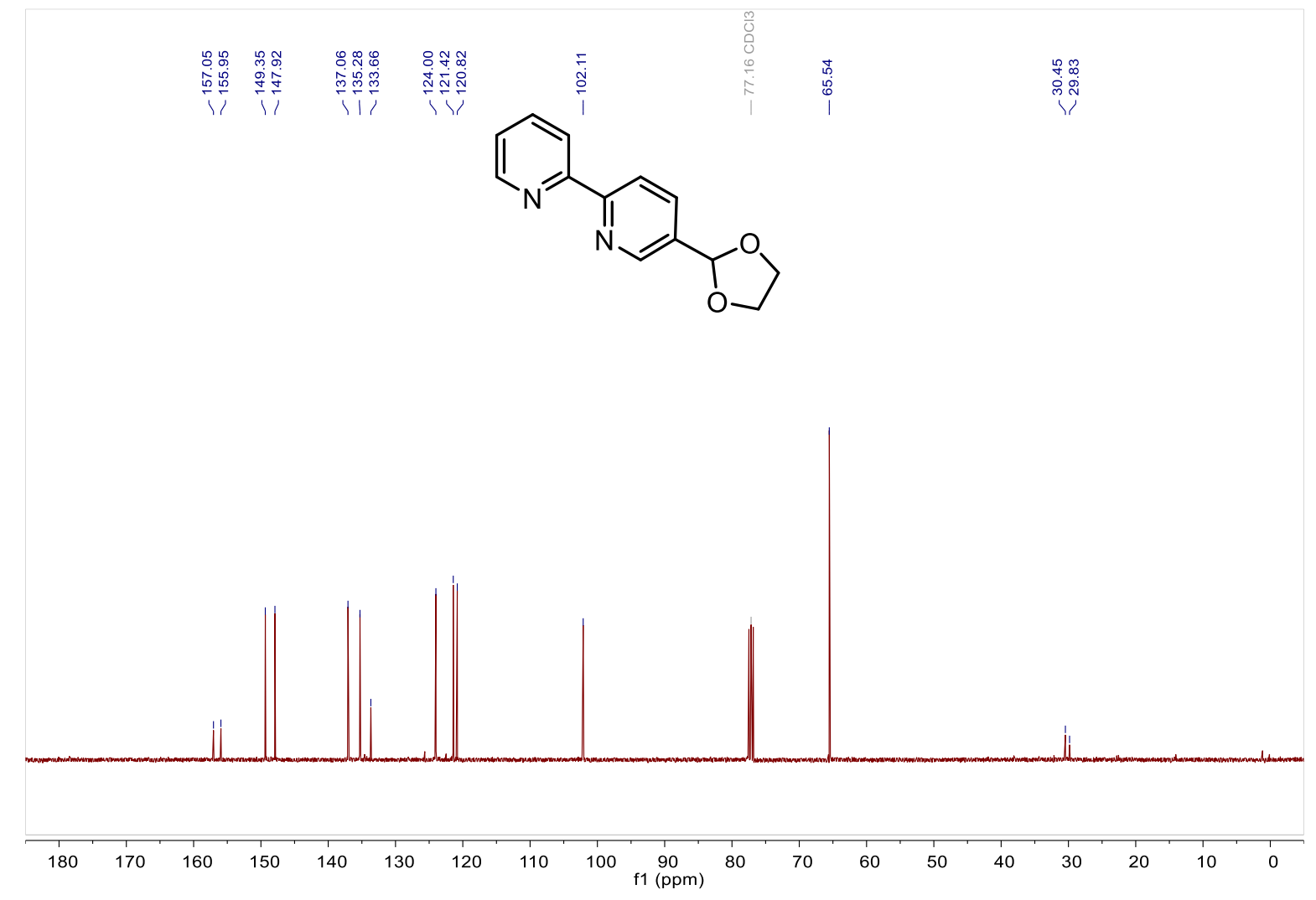


${ }^{1} \mathrm{H}$ NMR (500 MHz, $\mathrm{CDCl}_{3}$ ): 11

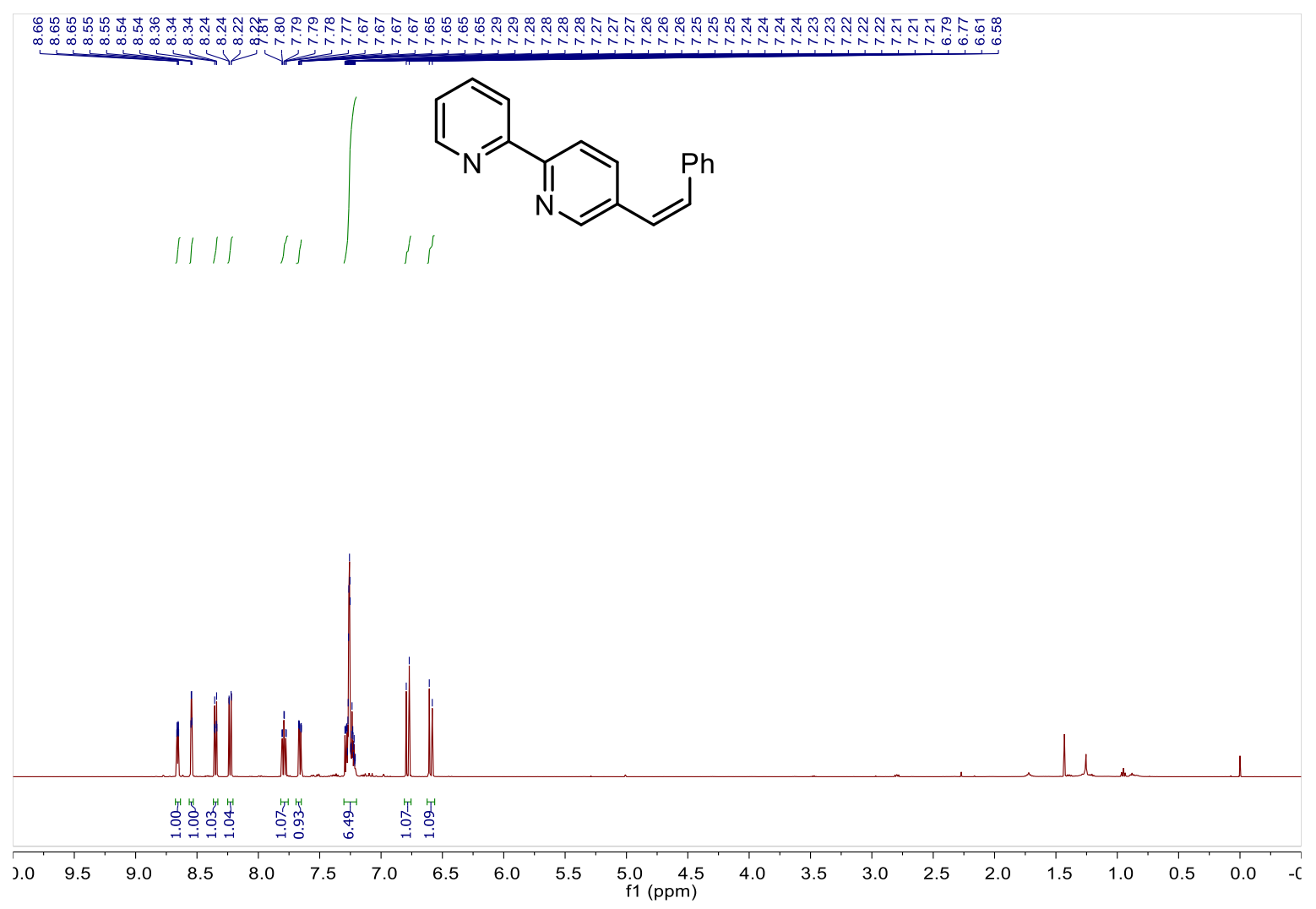

${ }^{13} \mathrm{C}$ NMR (126 MHz, $\left.\mathrm{CDCl}_{3}\right): 11$

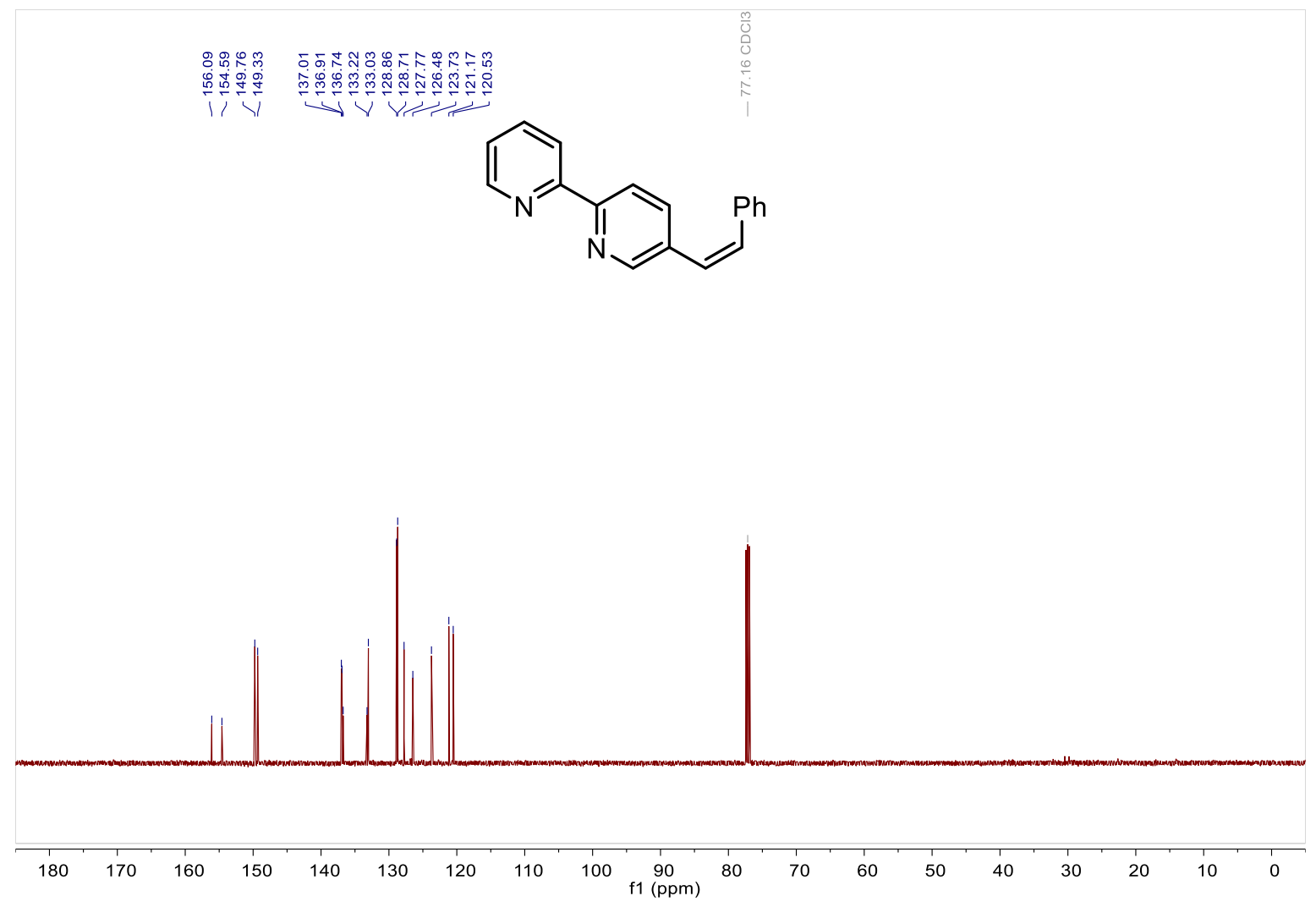

S65 
${ }^{1} \mathrm{H}$ NMR (500 MHz, $\left.\mathrm{CDCl}_{3}\right): 12$

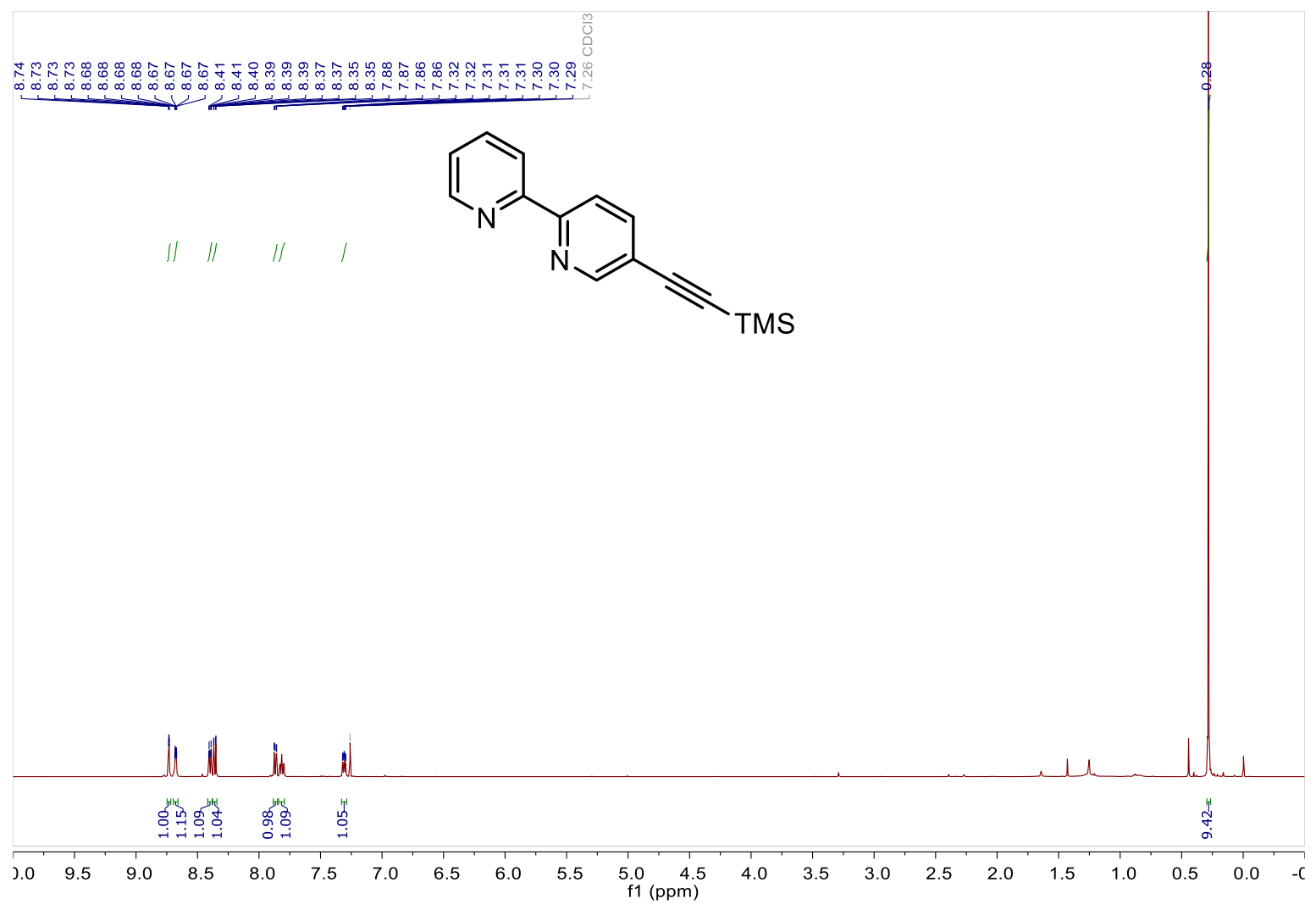

${ }^{13} \mathrm{C}$ NMR (101 MHz, $\left.\mathrm{CDCl}_{3}\right): 12$

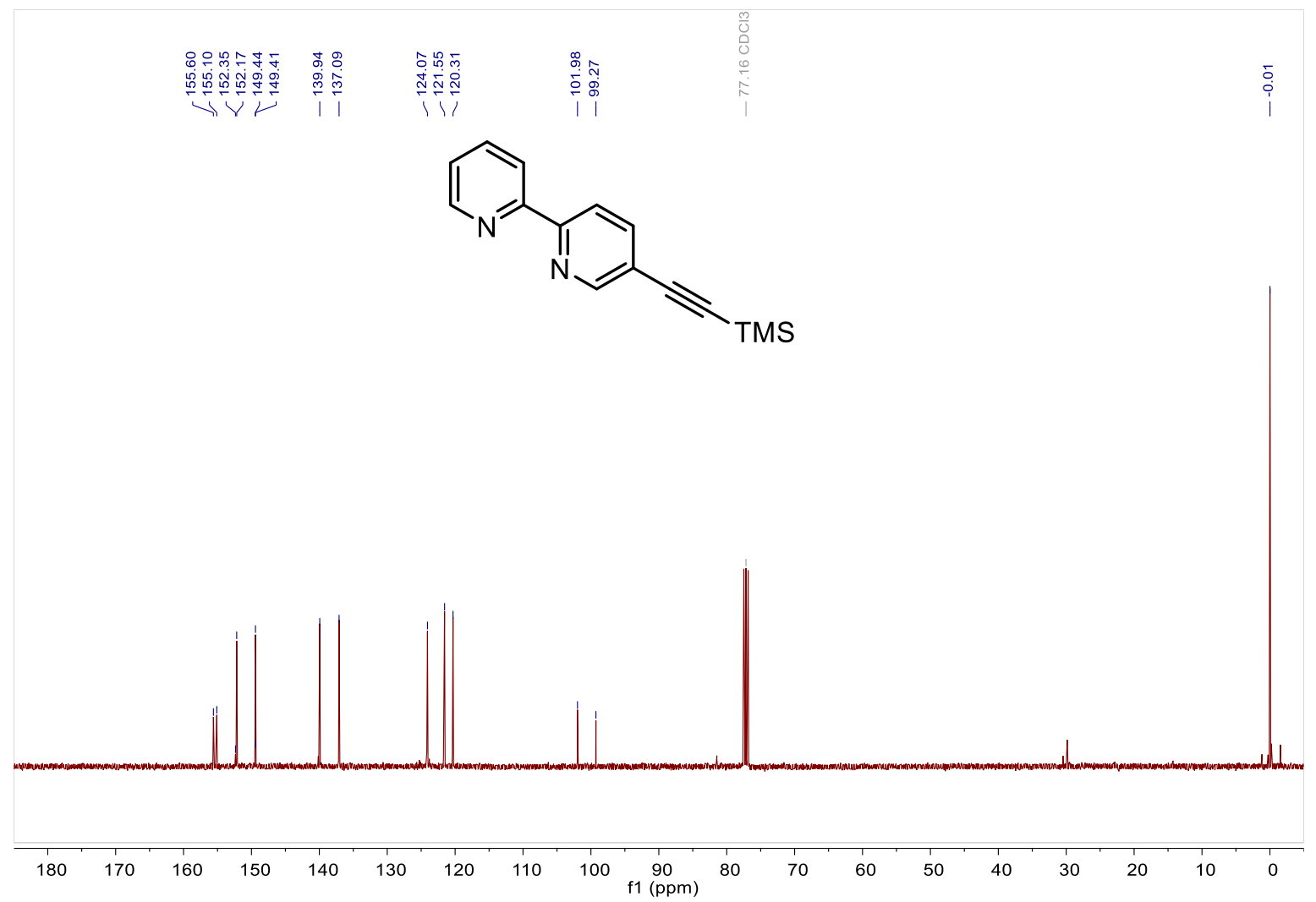

S66 
${ }^{1} \mathrm{H}$ NMR (300 MHz, $\mathrm{CDCl}_{3}$ ): 13

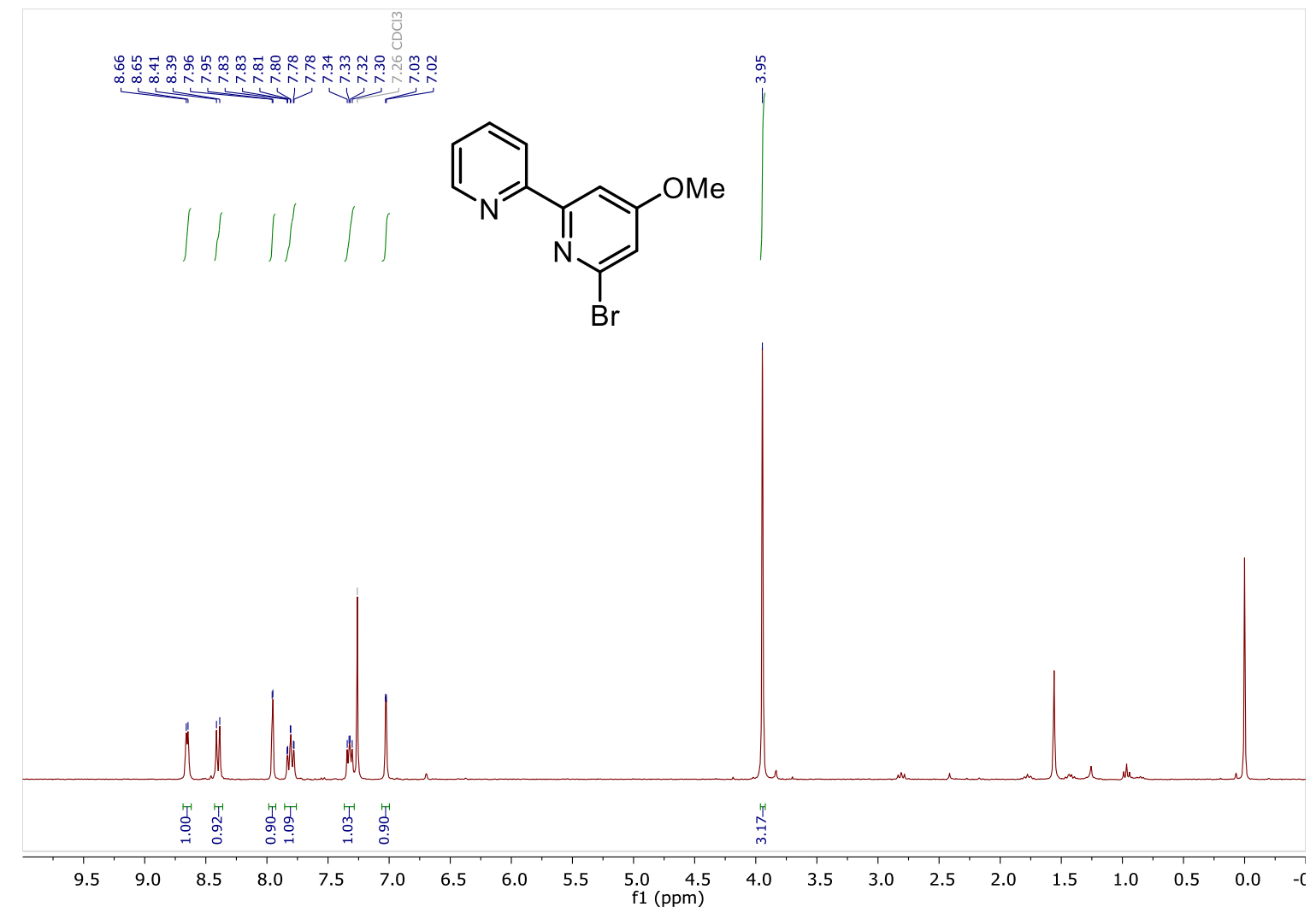

${ }^{13} \mathrm{C}$ NMR (126 MHz, $\left.\mathrm{CDCl}_{3}\right): 13$

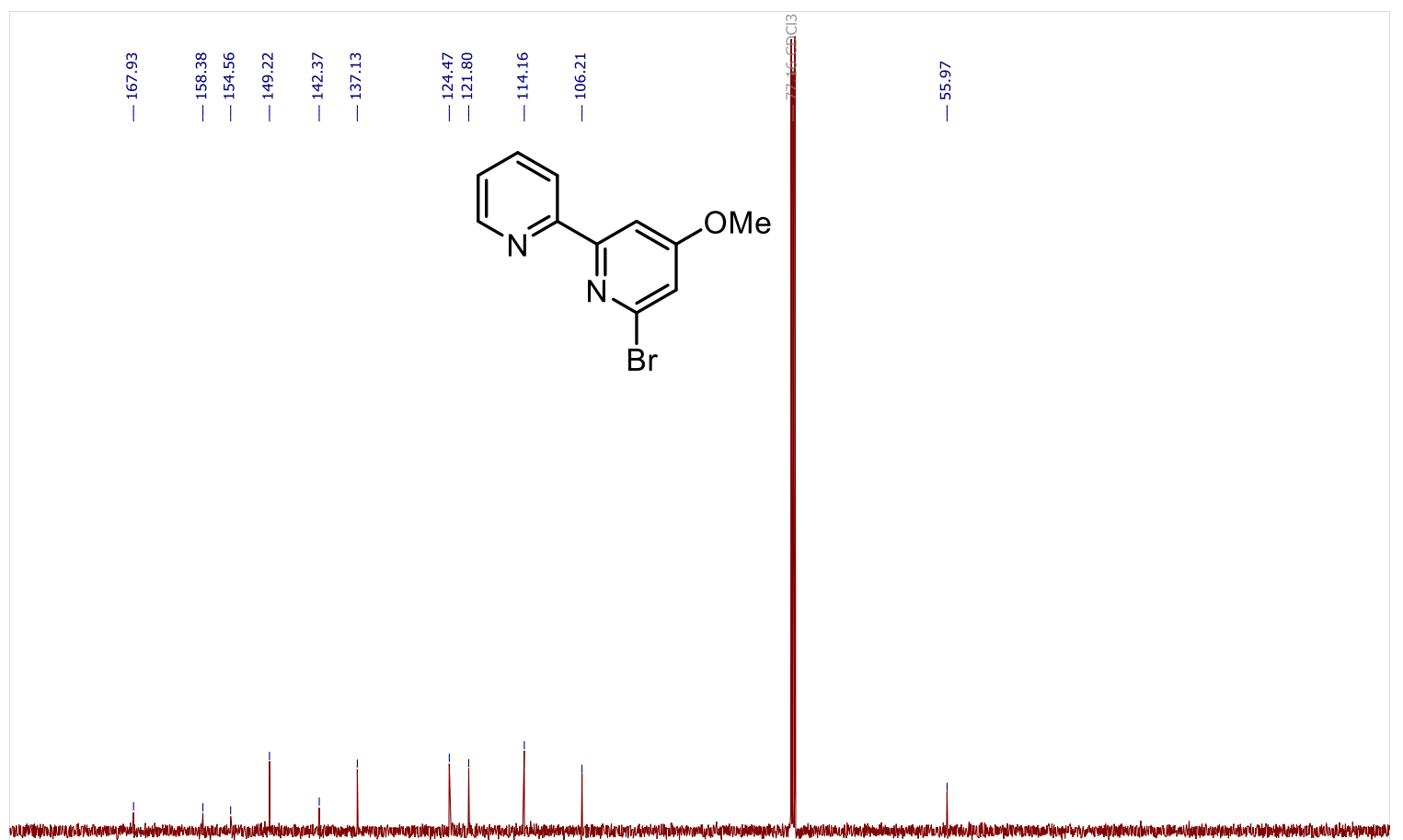

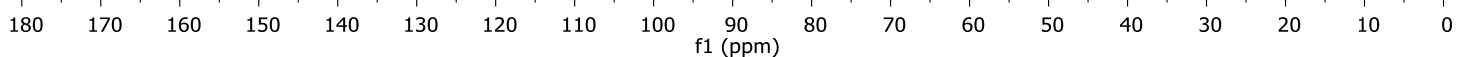


${ }^{1} \mathrm{H}$ NMR (400 MHz, $\mathrm{CDCl}_{3}$ ): 14

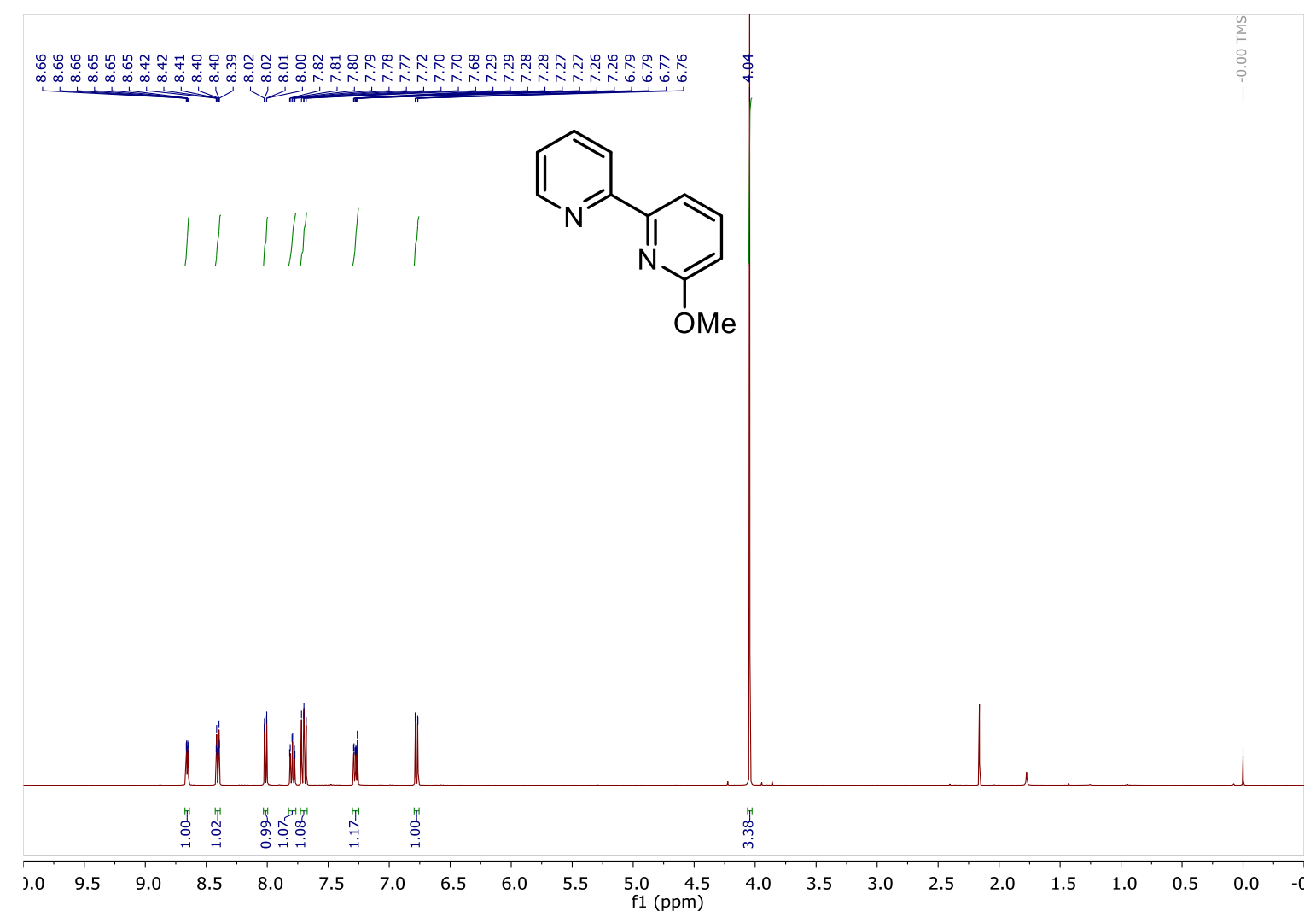

${ }^{13} \mathrm{C}$ NMR (101 MHz, $\left.\mathrm{CDCl}_{3}\right): 14$

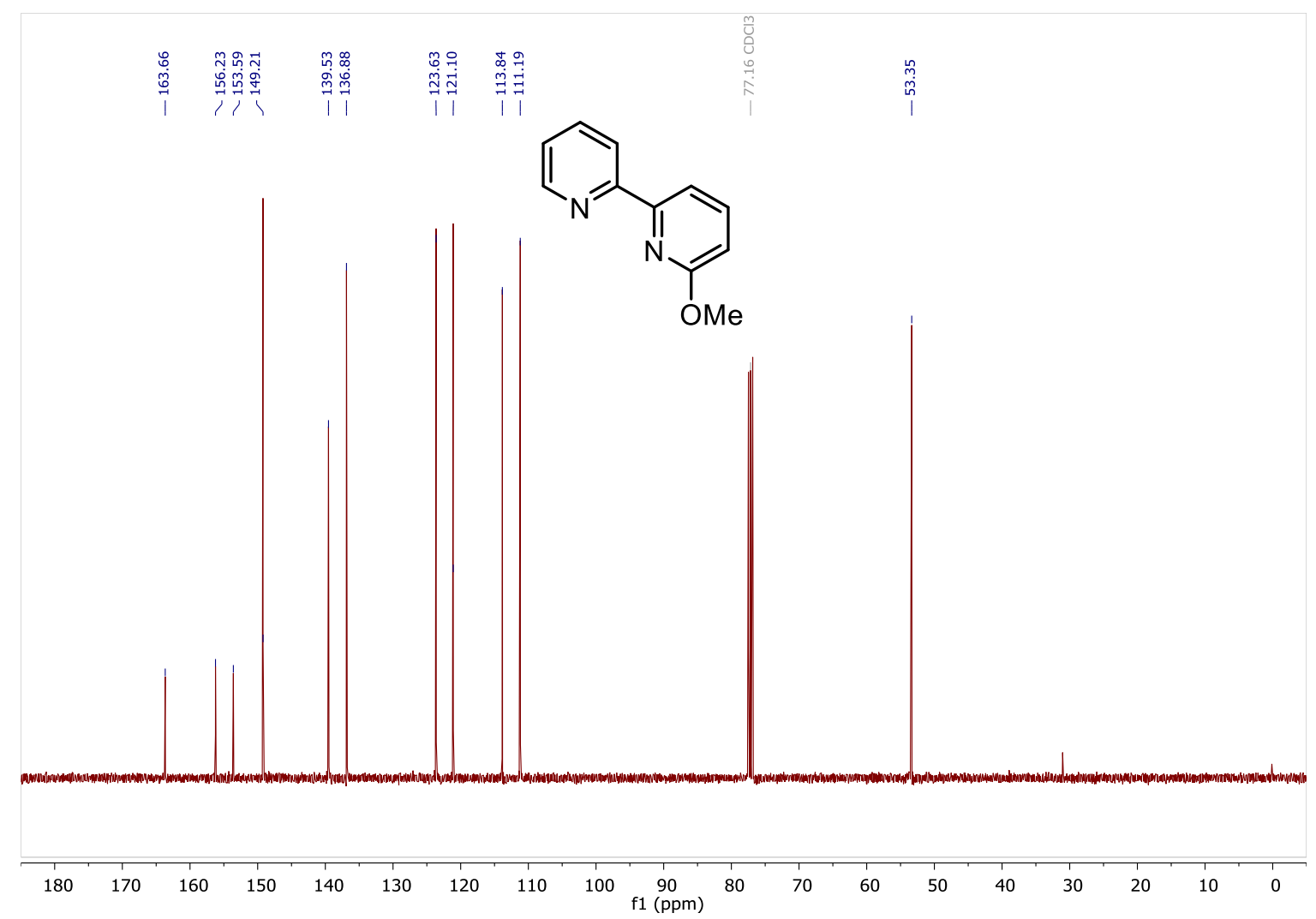


${ }^{1} \mathrm{H}$ NMR (500 MHz, $\mathrm{CDCl}_{3}$ ): 15

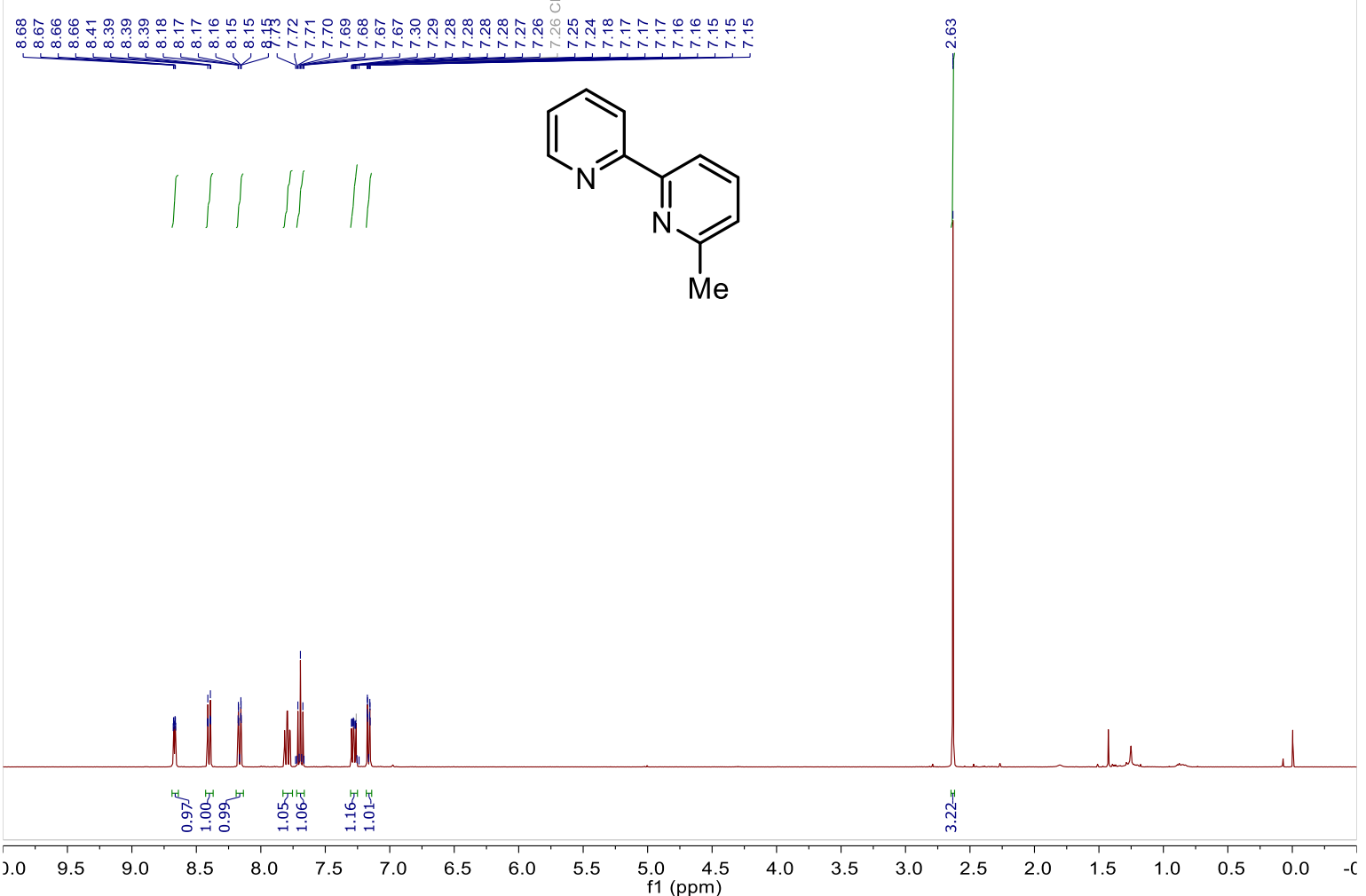

${ }^{13}$ C NMR (101 MHz, $\left.\mathrm{CDCl}_{3}\right): 15$

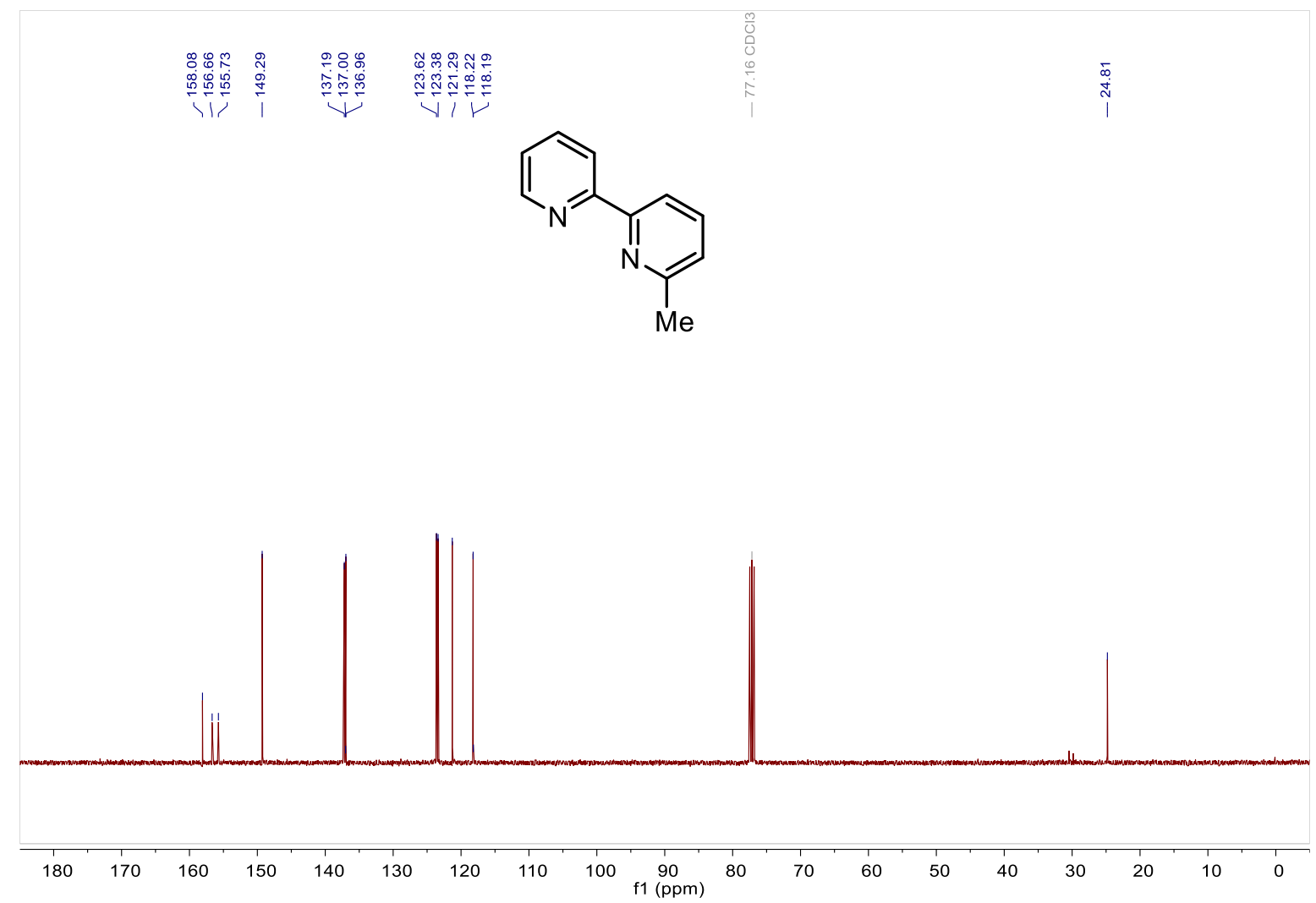


${ }^{1} \mathrm{H}$ NMR (500 MHz, $\mathrm{CDCl}_{3}$ ): 16

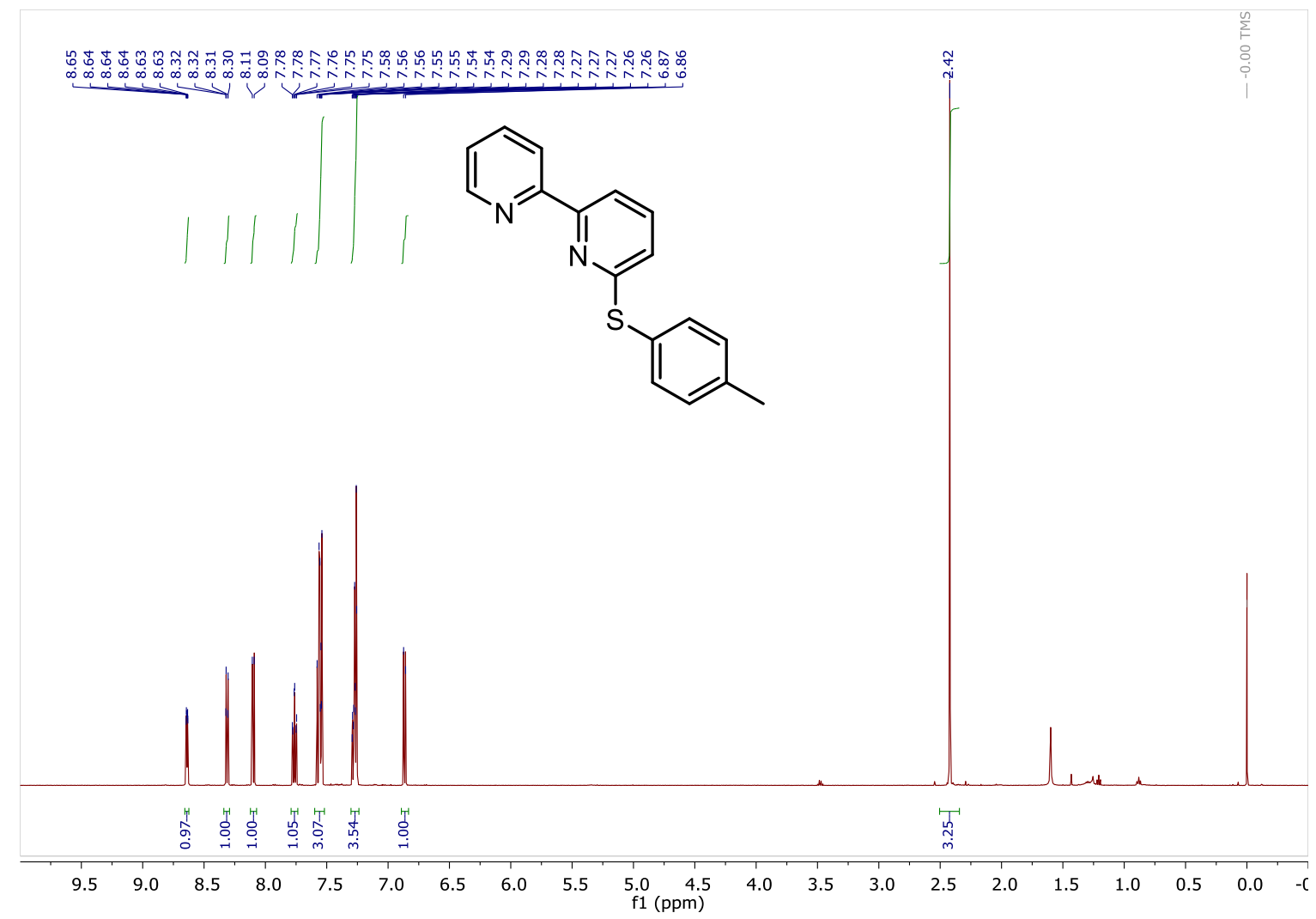

${ }^{13} \mathrm{C}$ NMR (126 MHz, $\left.\mathrm{CDCl}_{3}\right): 16$

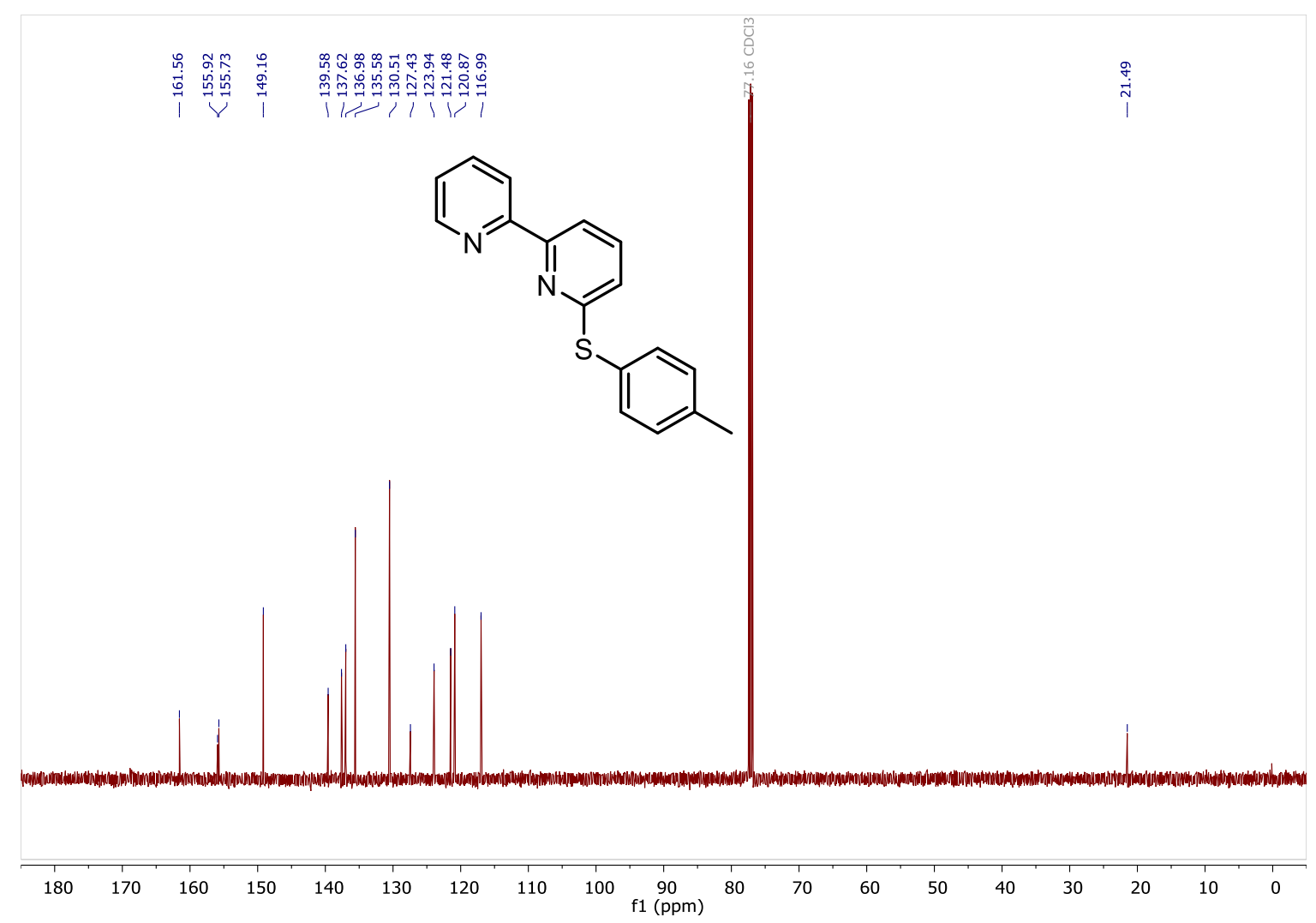


${ }^{1} \mathrm{H}$ NMR (500 MHz, $\mathrm{CDCl}_{3}$ ): 17

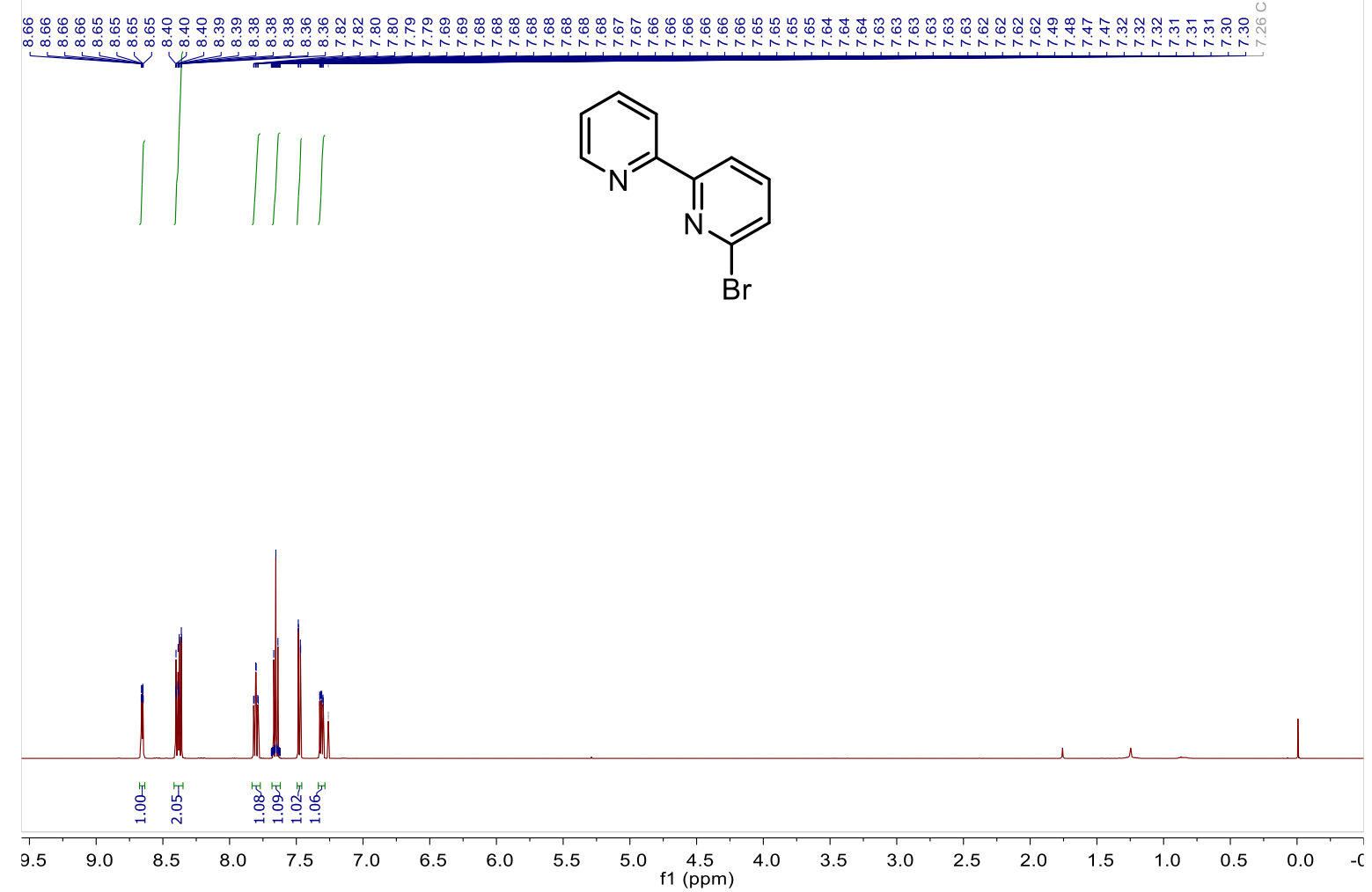

${ }^{13} \mathrm{C}$ NMR (126 MHz, $\left.\mathrm{CDCl}_{3}\right): 17$

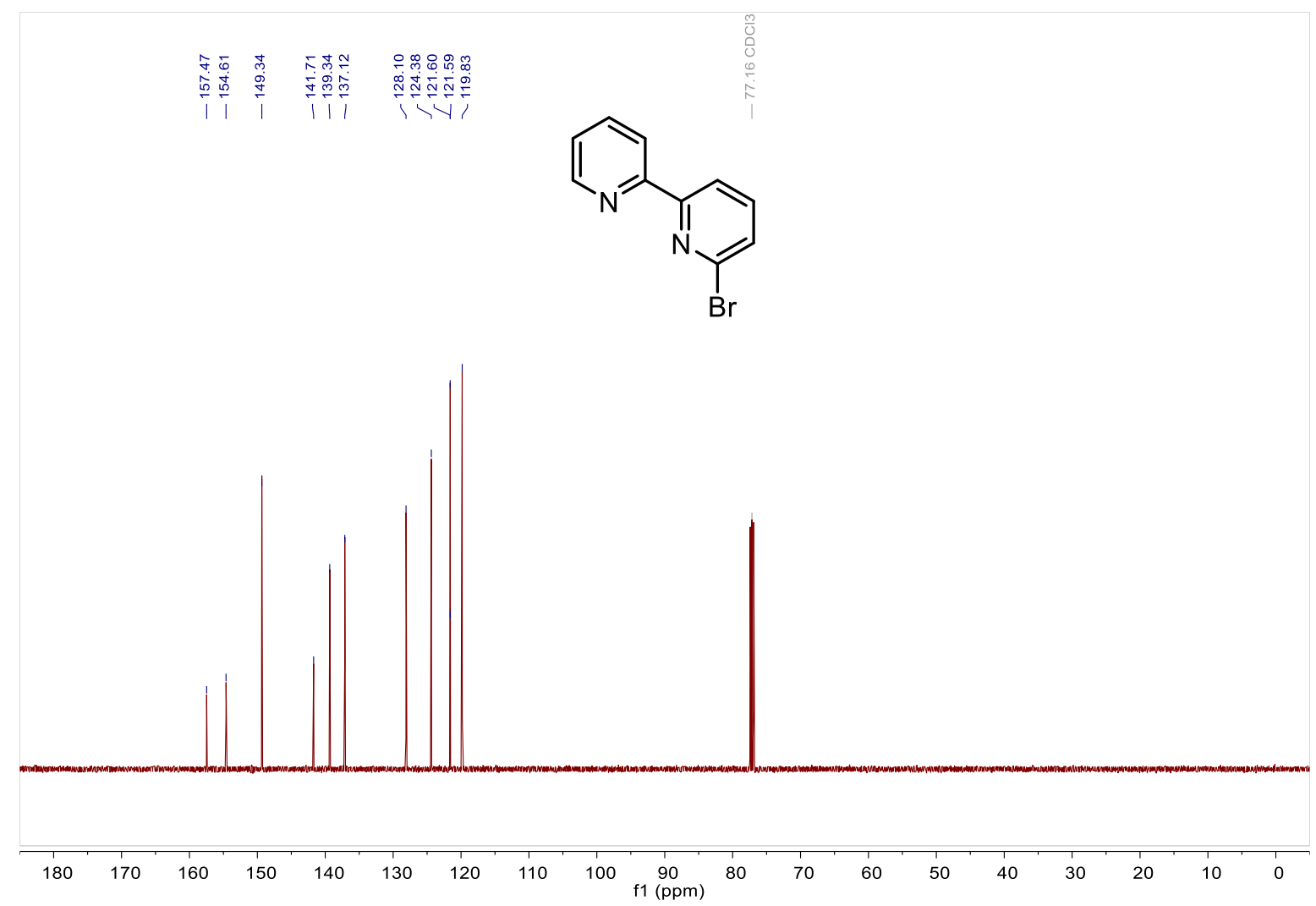


${ }^{1} \mathrm{H}$ NMR (500 MHz, $\left.\mathrm{CDCl}_{3}\right): 18$

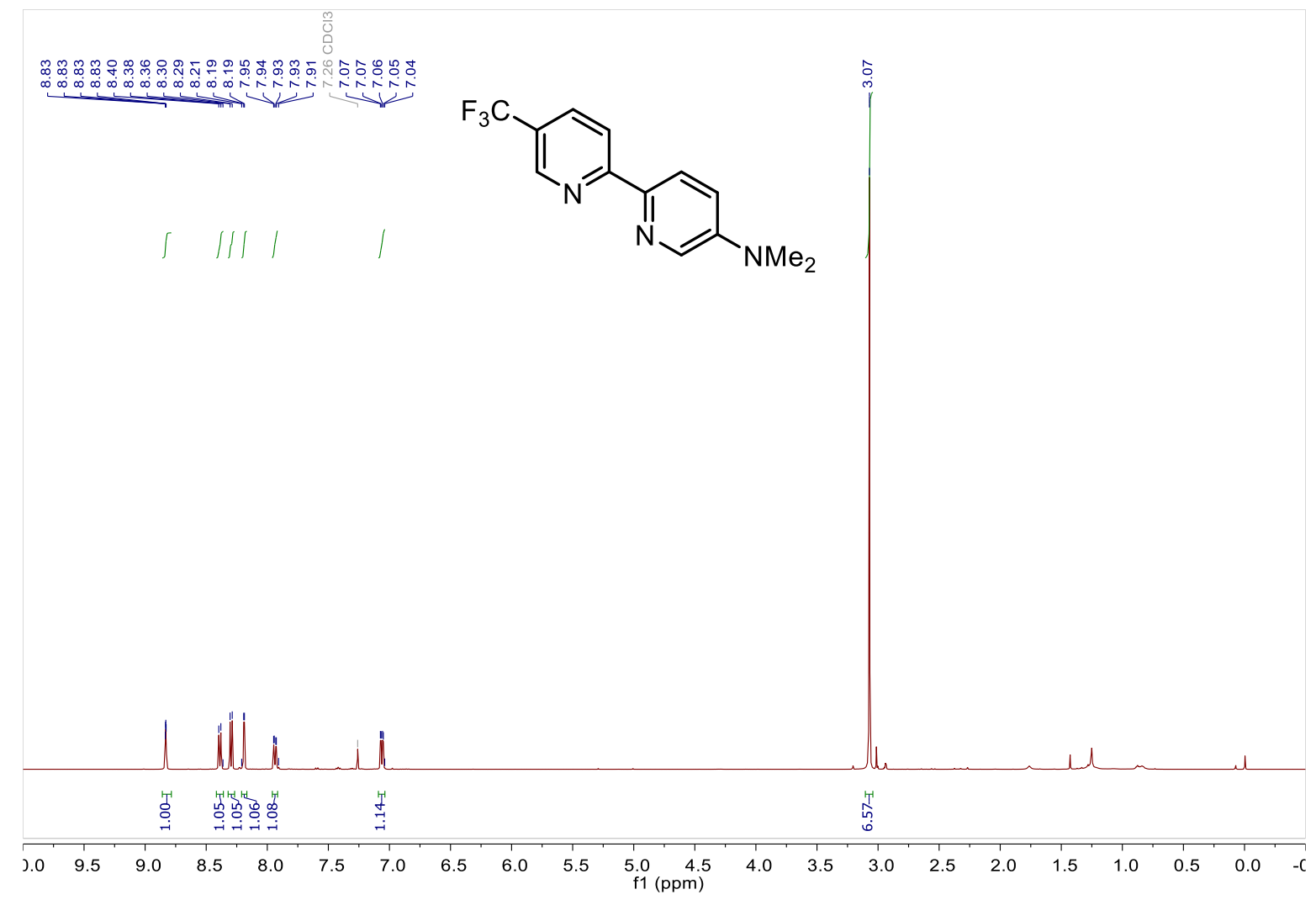

${ }^{13} \mathrm{C}$ NMR (126 MHz, $\left.\mathrm{CDCl}_{3}\right): 18$

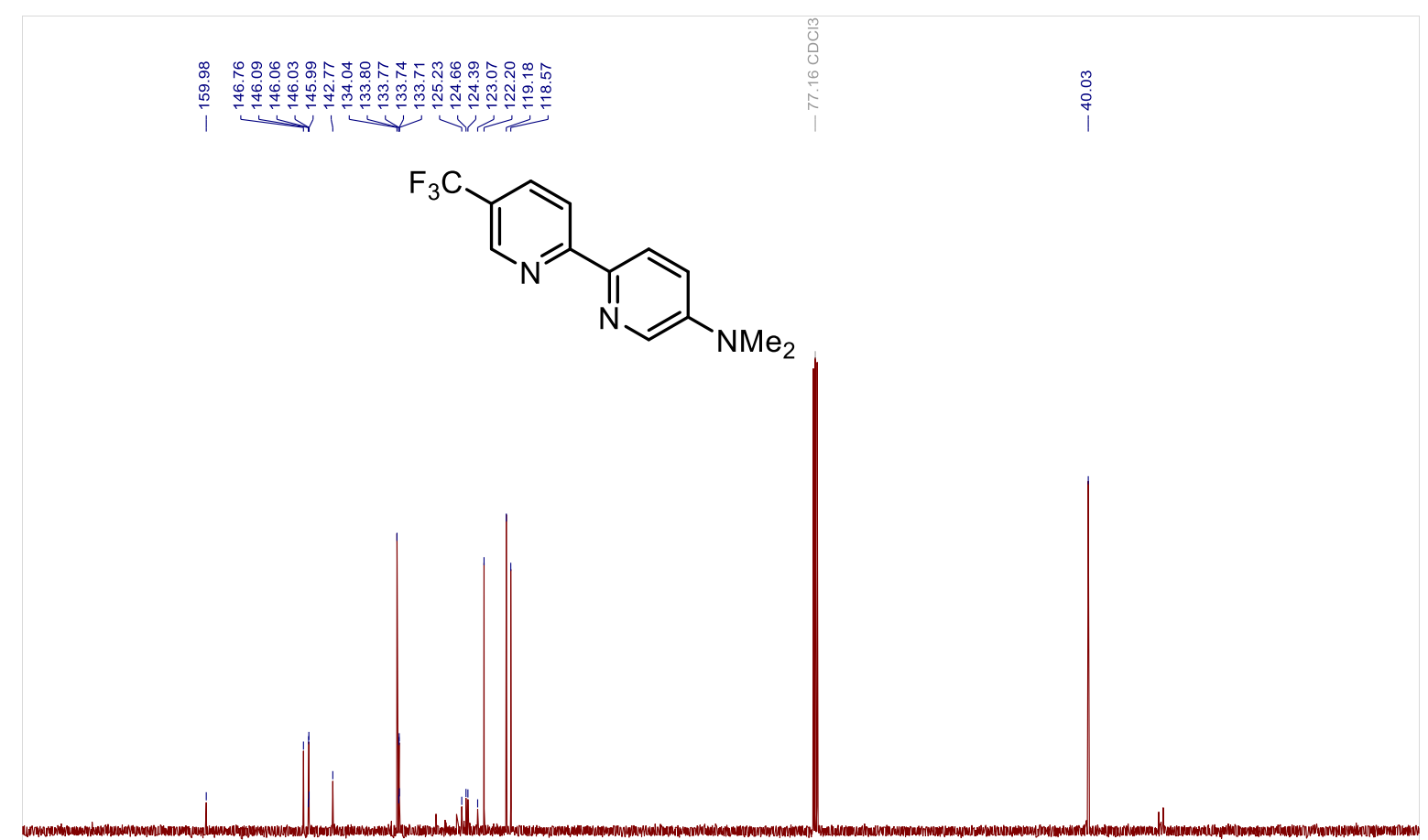

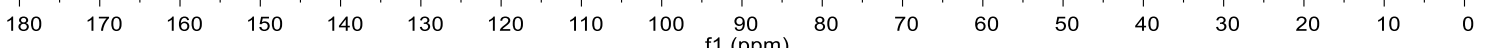


${ }^{1} \mathrm{H}$ NMR (400 MHz, $\left.\mathrm{CDCl}_{3}\right): 19$

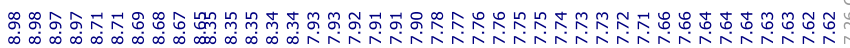<smiles>FC(F)(F)c1ccnc(-c2nccc3ccccc23)c1</smiles>

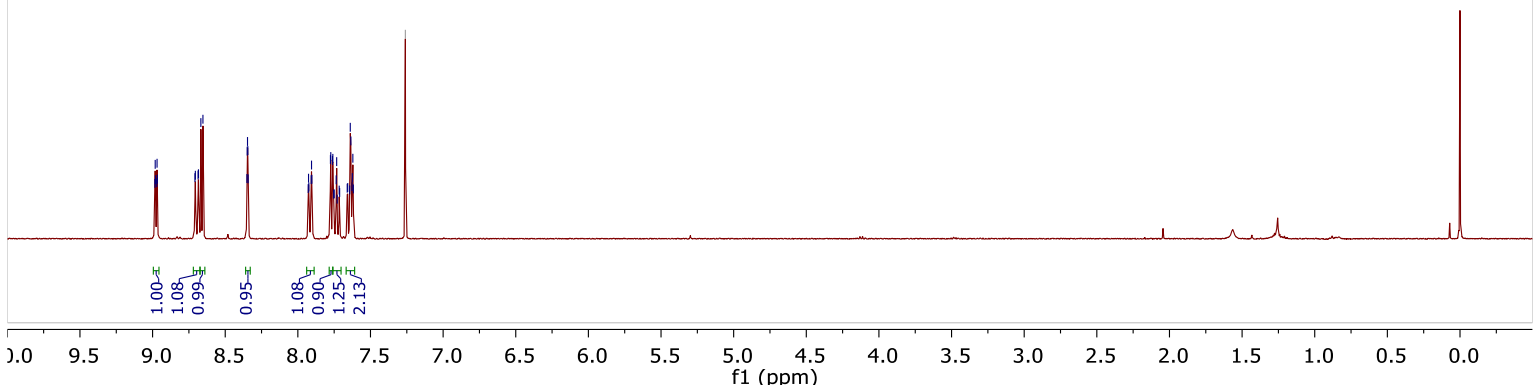

${ }^{13}$ C NMR (101 MHz, $\left.\mathrm{CDCl}_{3}\right): 19$

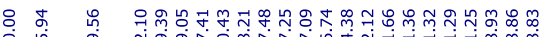

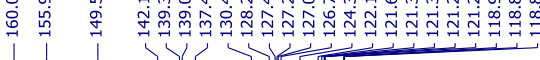<smiles>FC(F)(F)c1ccnc(-c2nccc3ccccc23)c1</smiles>

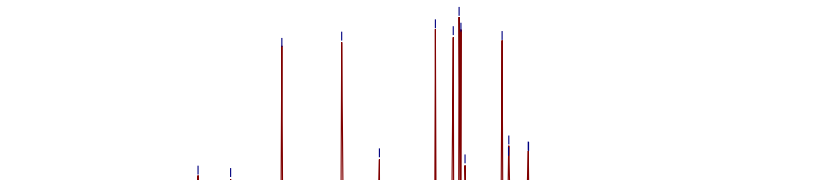

$\begin{array}{llllllllll}180 & 170 & 160 & 150 & 140 & 130 & 120 & 110 & 100 & \underset{f 1}{(\mathrm{ppm})} \\ 80\end{array}$ 
${ }^{1} \mathrm{H}$ NMR (500 MHz, $\mathrm{CDCl}_{3}$ ): 20

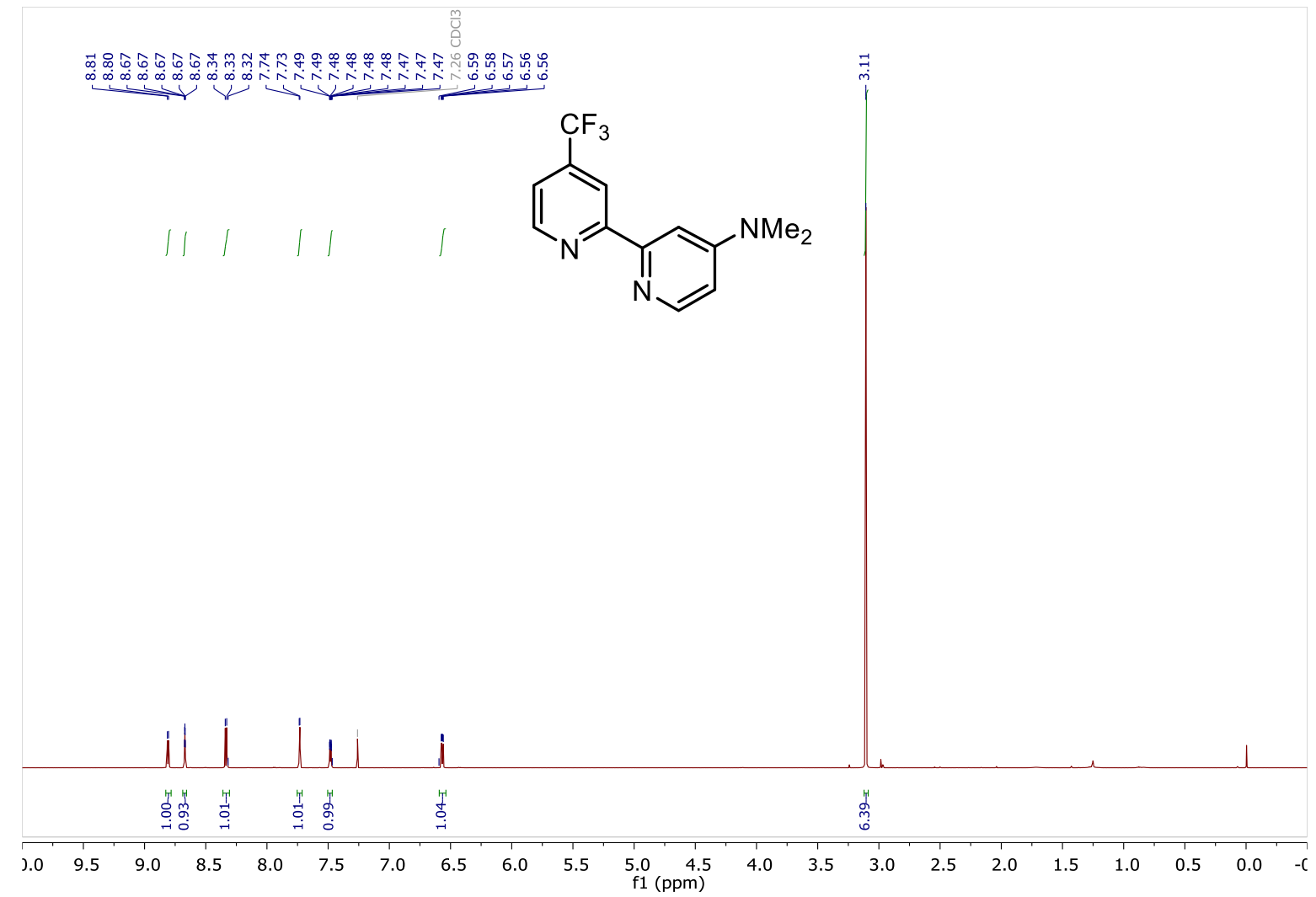

${ }^{13} \mathrm{C}$ NMR (126 MHz, $\left.\mathrm{CDCl}_{3}\right): 20$

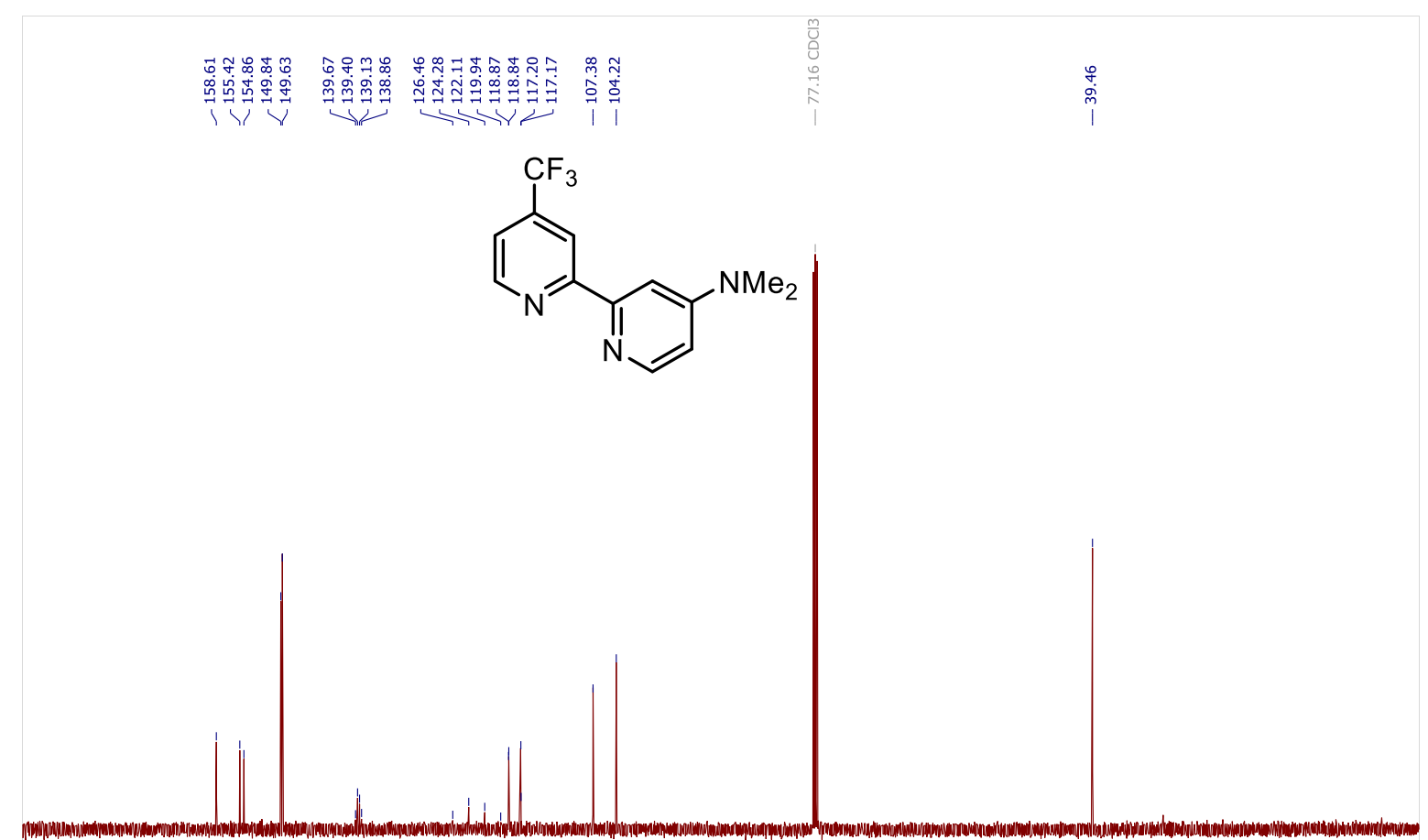

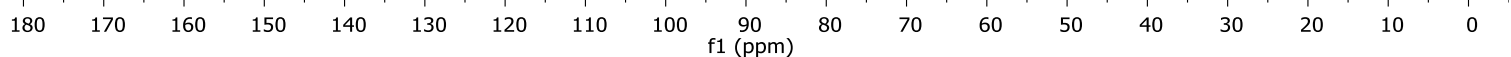


${ }^{1} \mathrm{H}$ NMR (500 MHz, $\mathrm{CDCl}_{3}$ ): 21

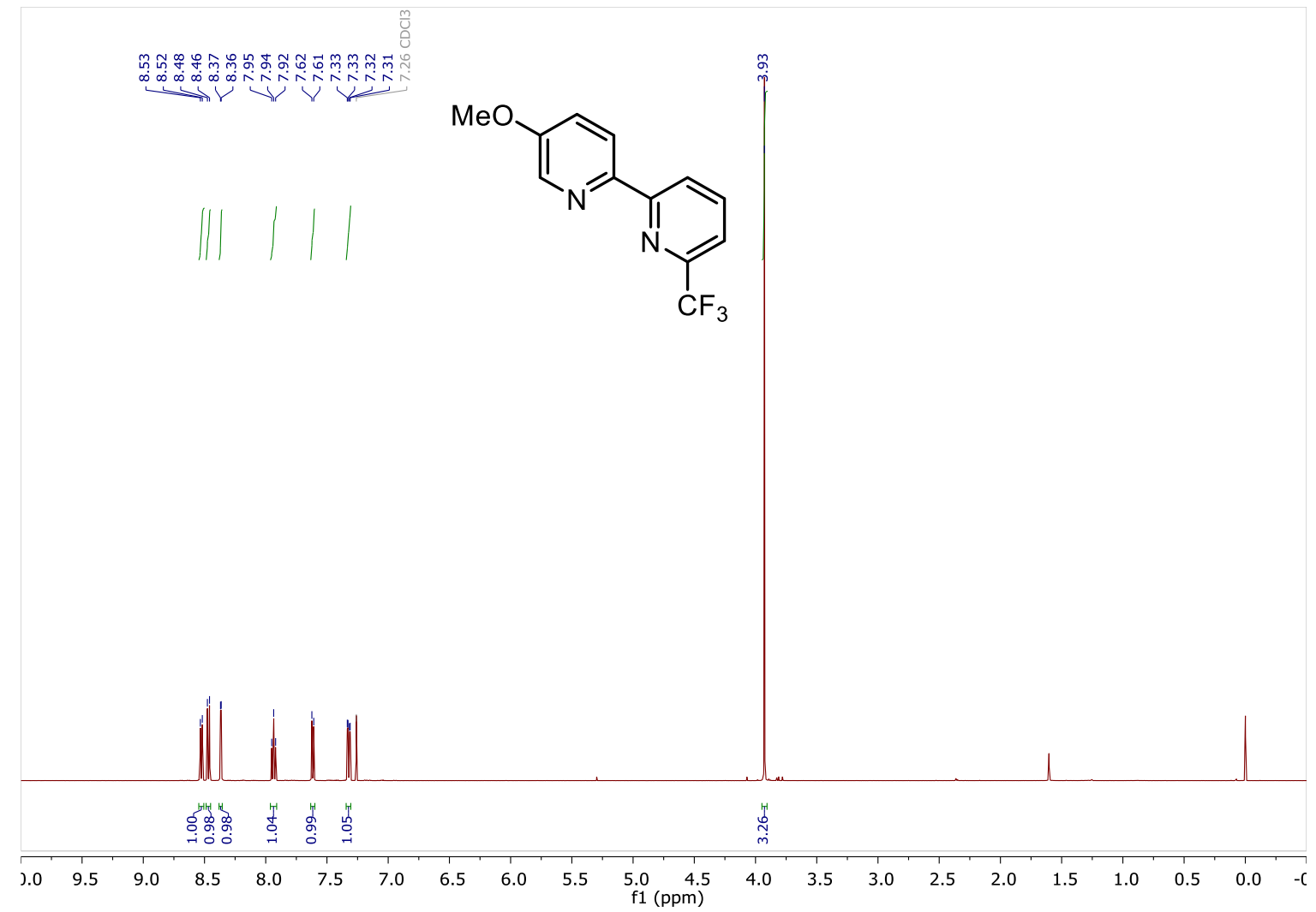

${ }^{13} \mathrm{C}$ NMR (126 MHz, $\left.\mathrm{CDCl}_{3}\right): 21$

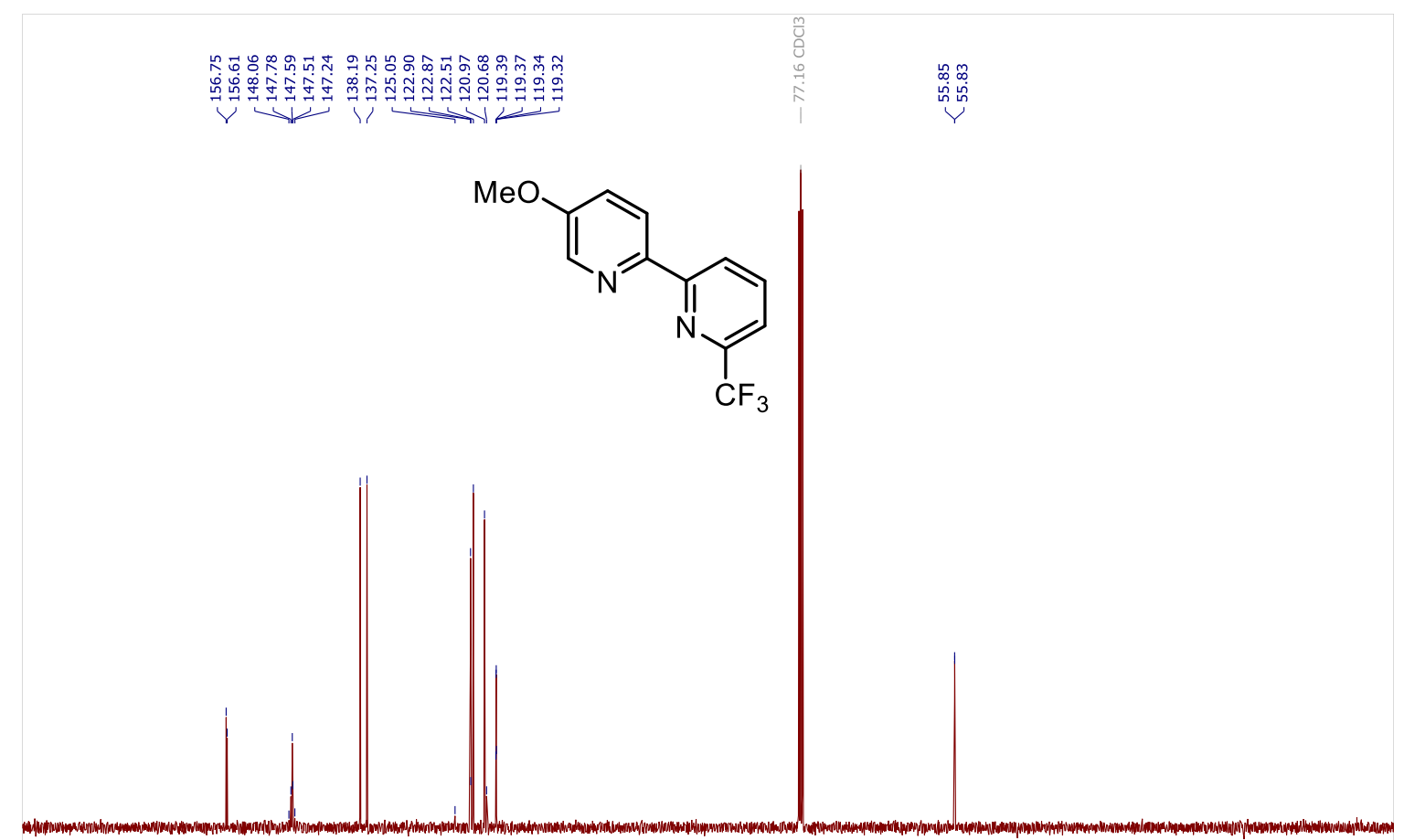

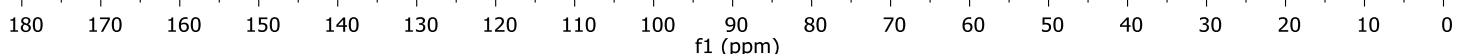


${ }^{1} \mathrm{H}$ NMR (400 MHz, $\mathrm{CDCl}_{3}$ ): 22

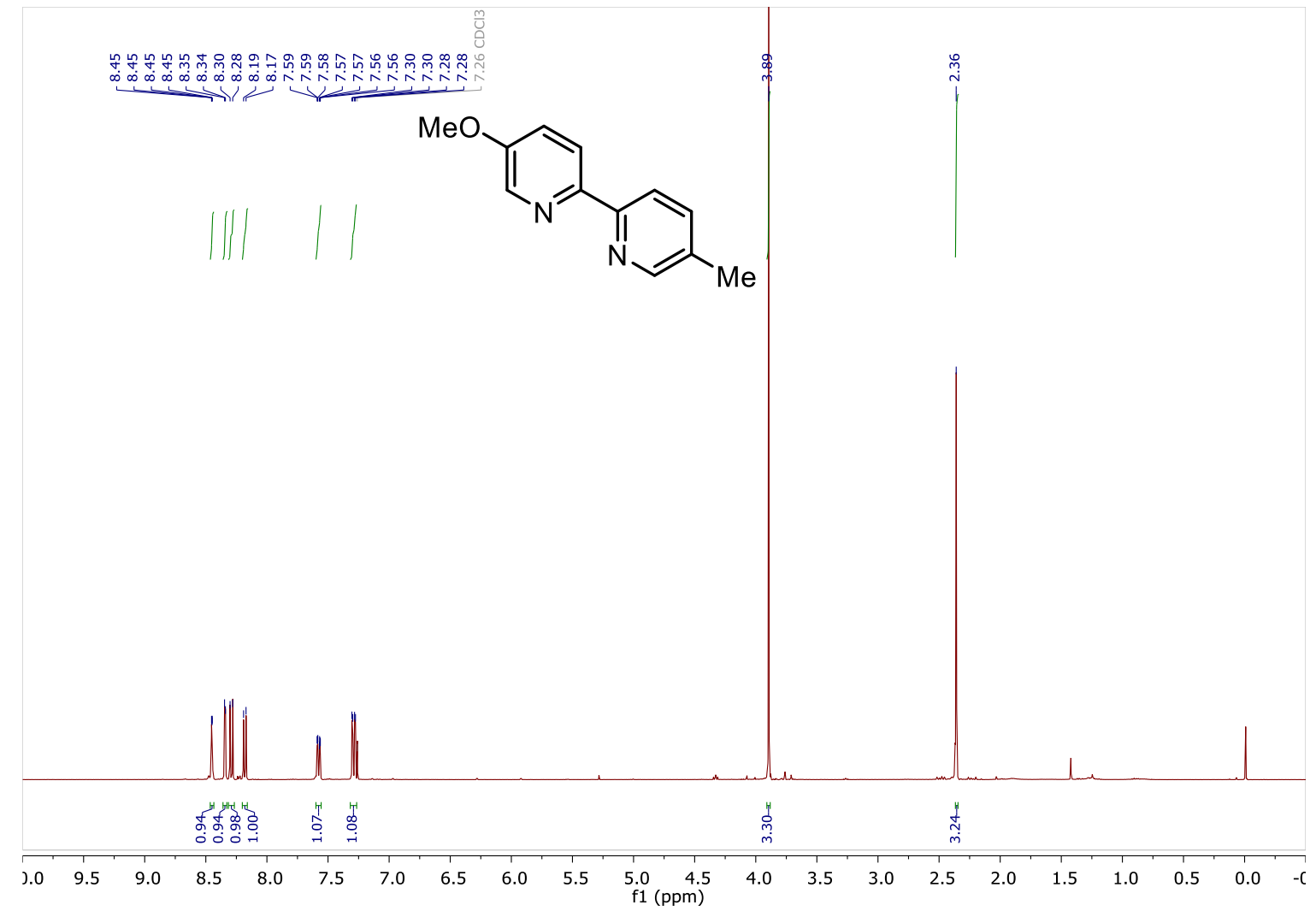

${ }^{13} \mathrm{C}$ NMR (101 MHz, $\left.\mathrm{CDCl}_{3}\right): 22$

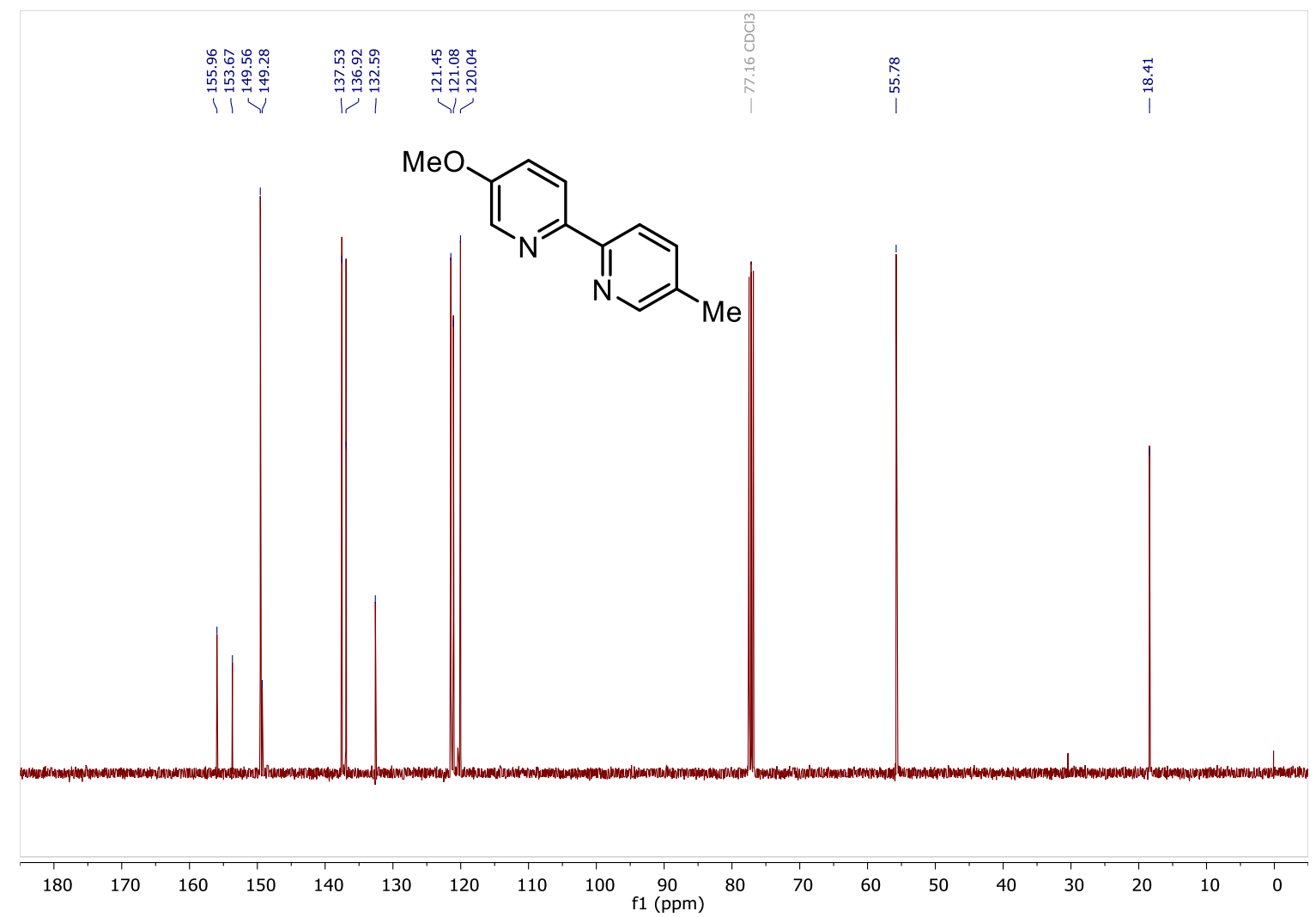


${ }^{1} \mathrm{H}$ NMR (400 MHz, $\left.\mathrm{CDCl}_{3}\right): 23$

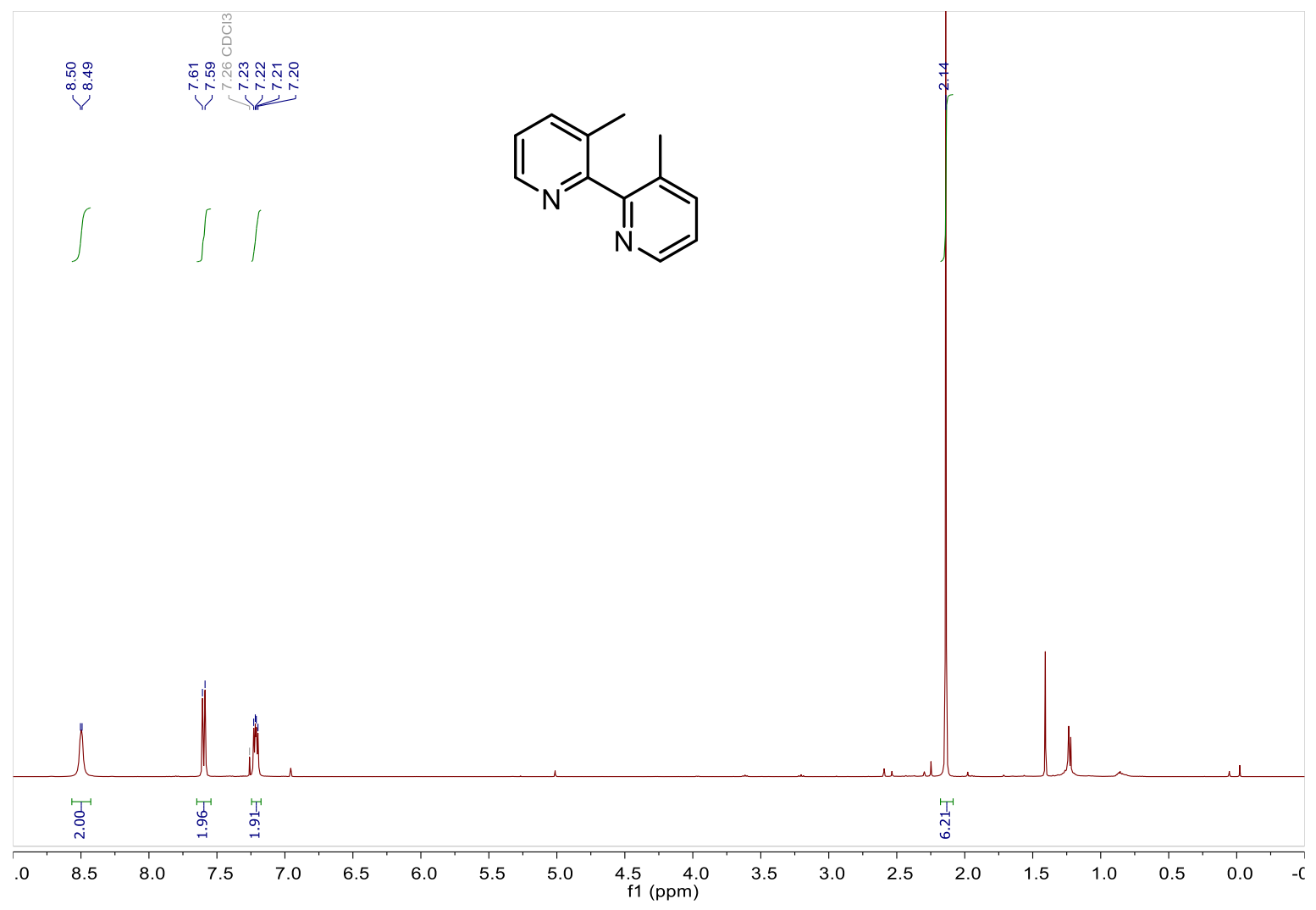

${ }^{13} \mathrm{C}$ NMR (101 MHz, $\left.\mathrm{CDCl}_{3}\right): 23$

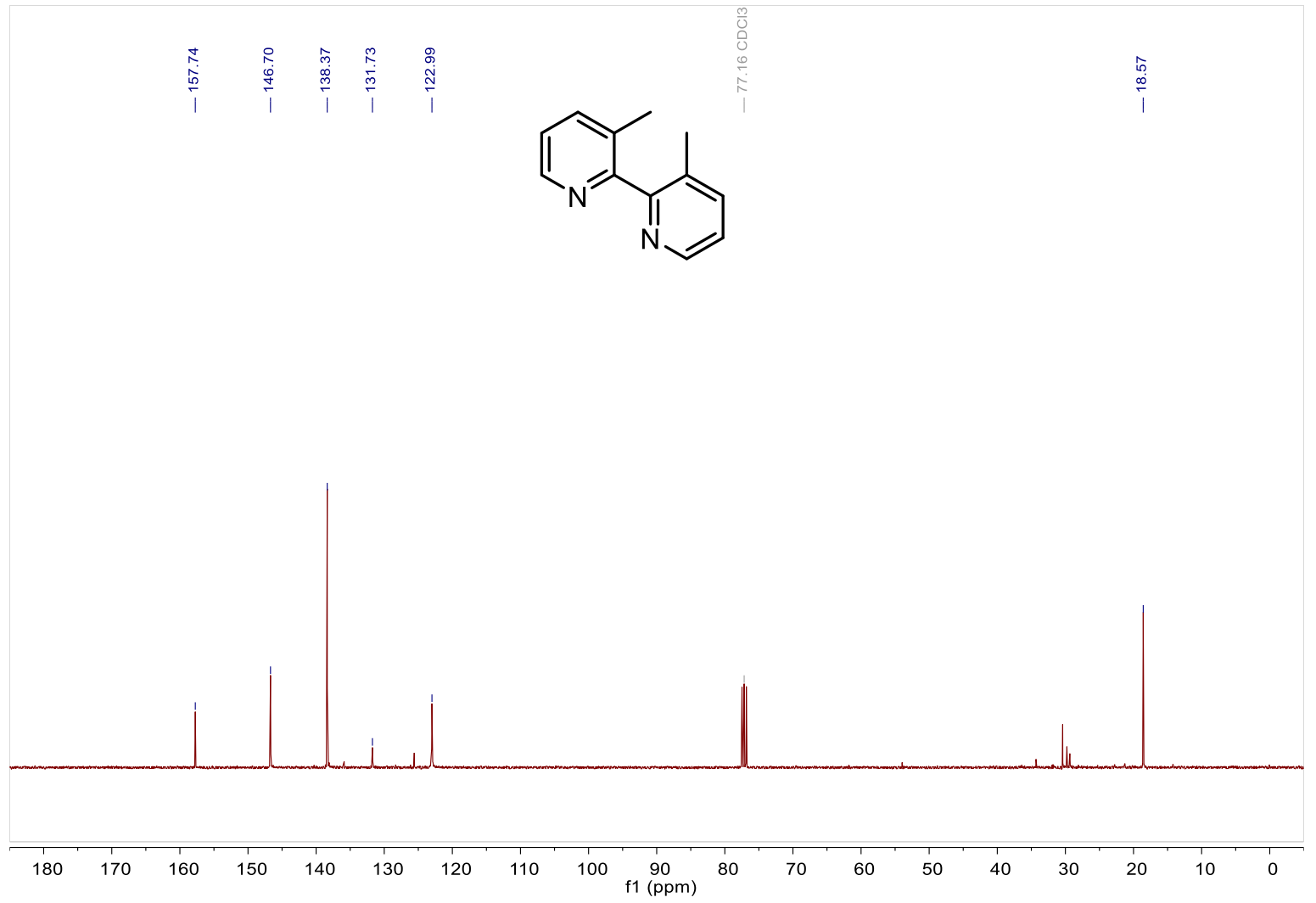

S77 
${ }^{1} \mathrm{H}$ NMR (500 MHz, $\mathrm{CDCl}_{3}$ ): 24

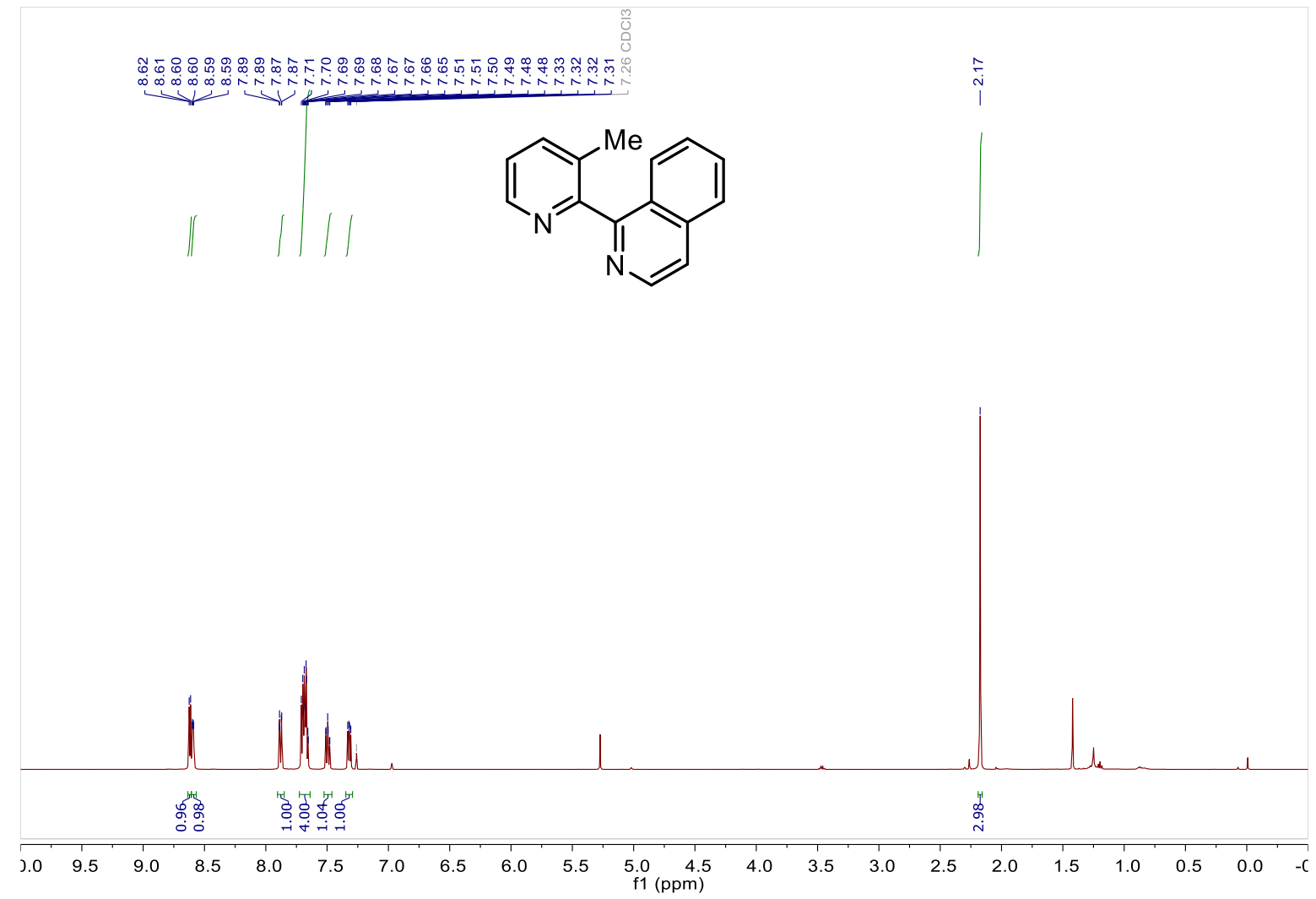

${ }^{13} \mathrm{C}$ NMR (126 MHz, $\left.\mathrm{CDCl}_{3}\right): 24$

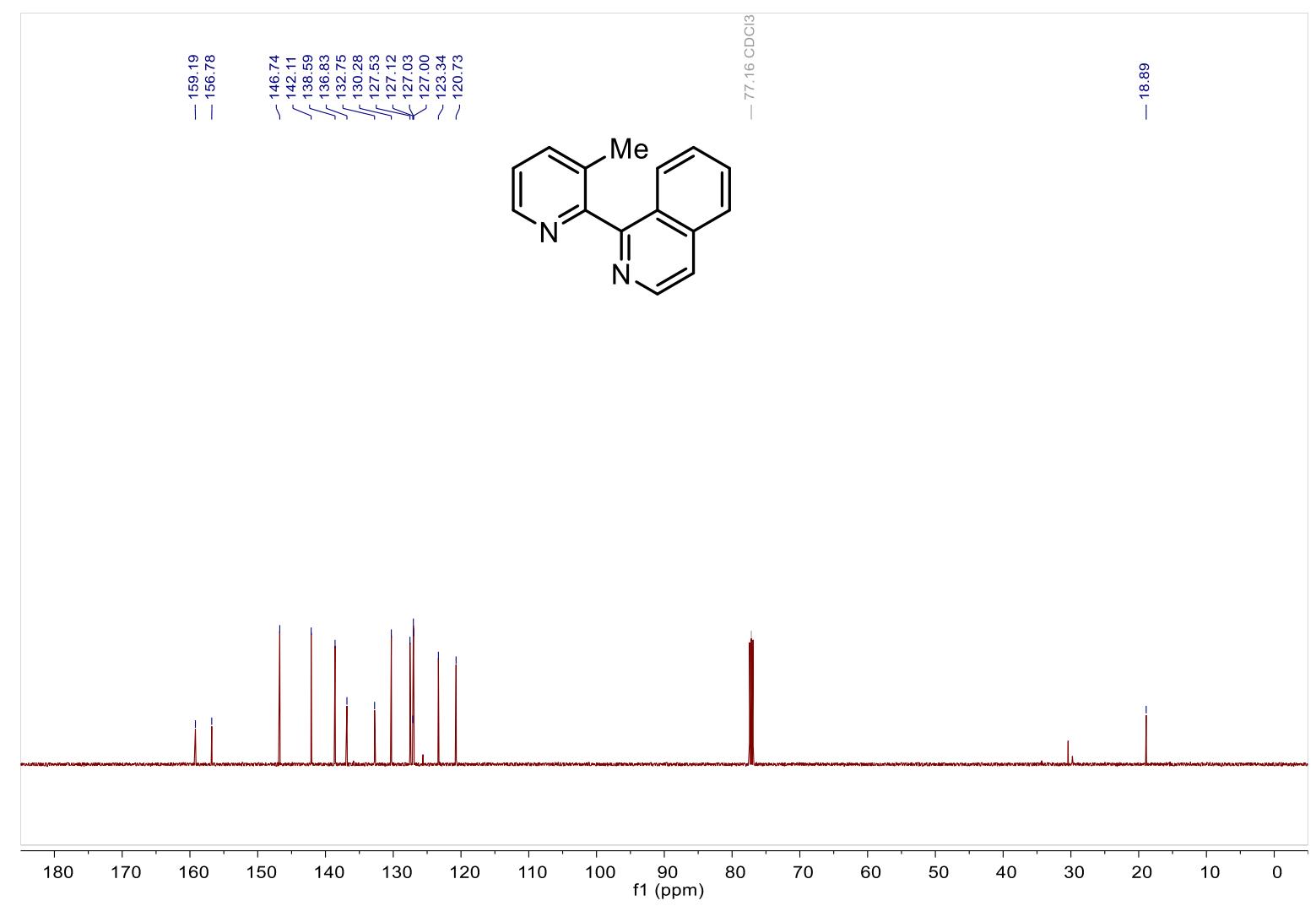


${ }^{1} \mathrm{H}$ NMR (400 MHz, $\mathrm{CDCl}_{3}$ ): 25

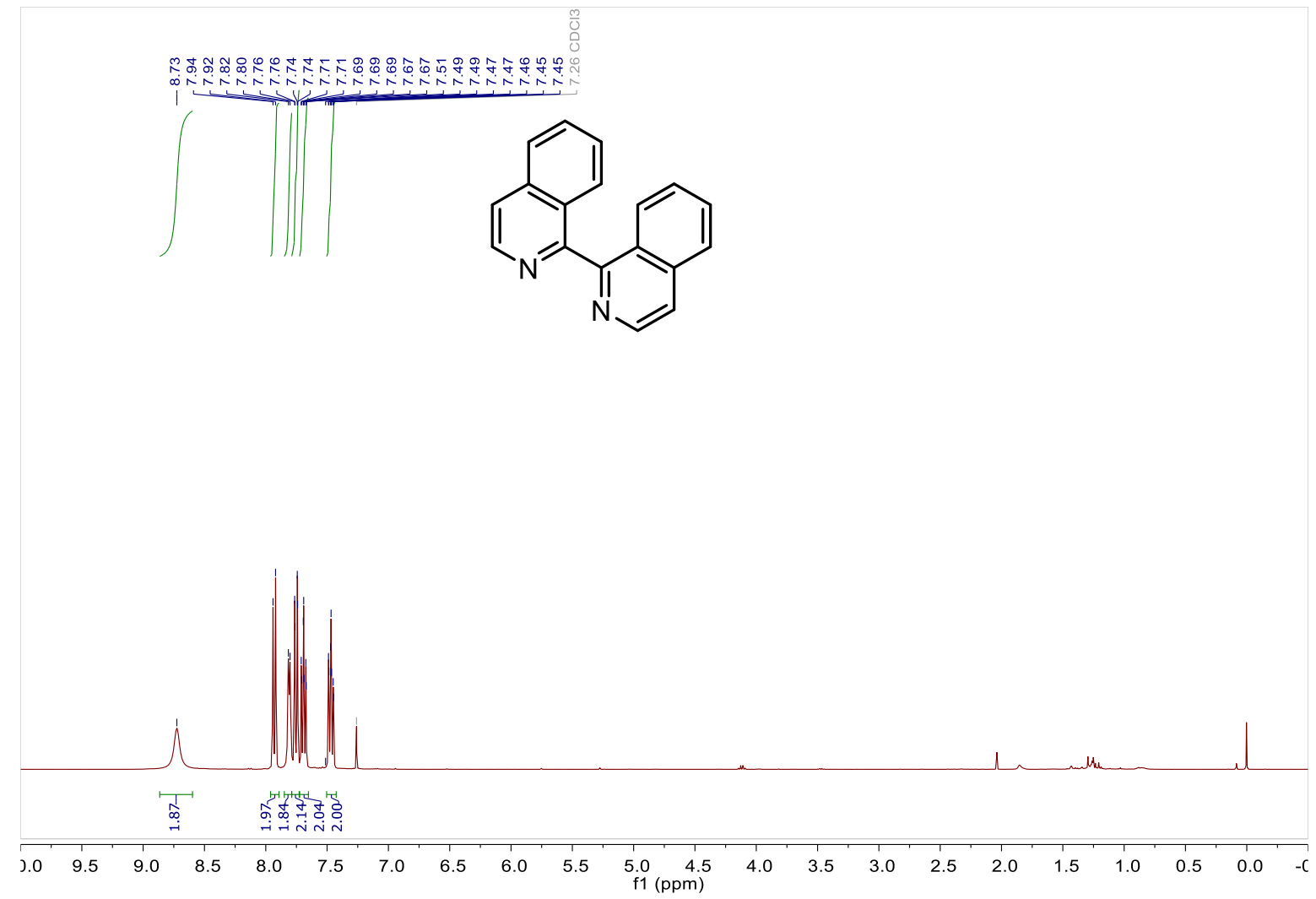

${ }^{13} \mathrm{C}$ NMR (101 MHz, $\left.\mathrm{CDCl}_{3}\right): 25$

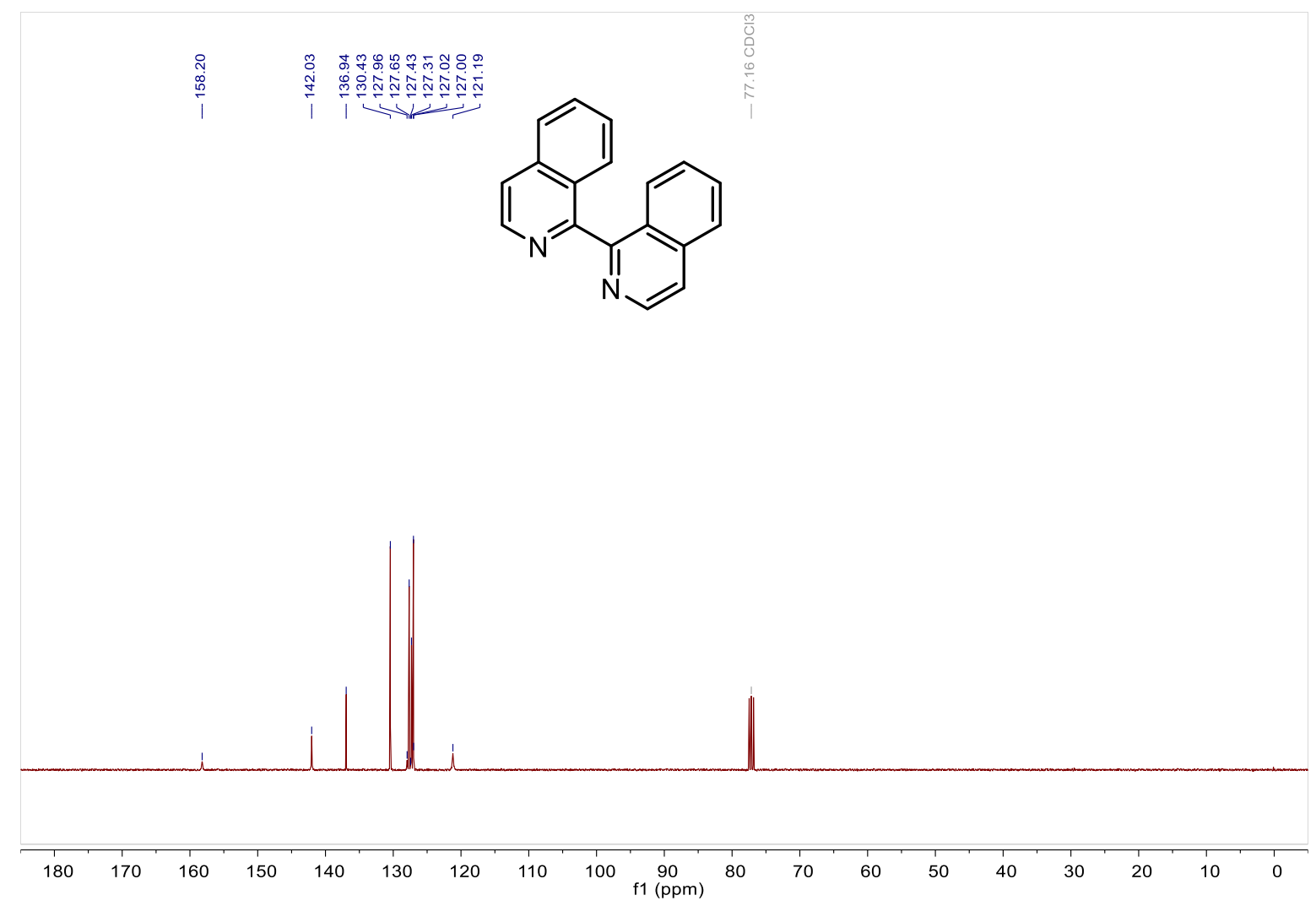


${ }^{1} \mathrm{H}$ NMR (500 MHz, $\mathrm{CDCl}_{3}$ ): 26

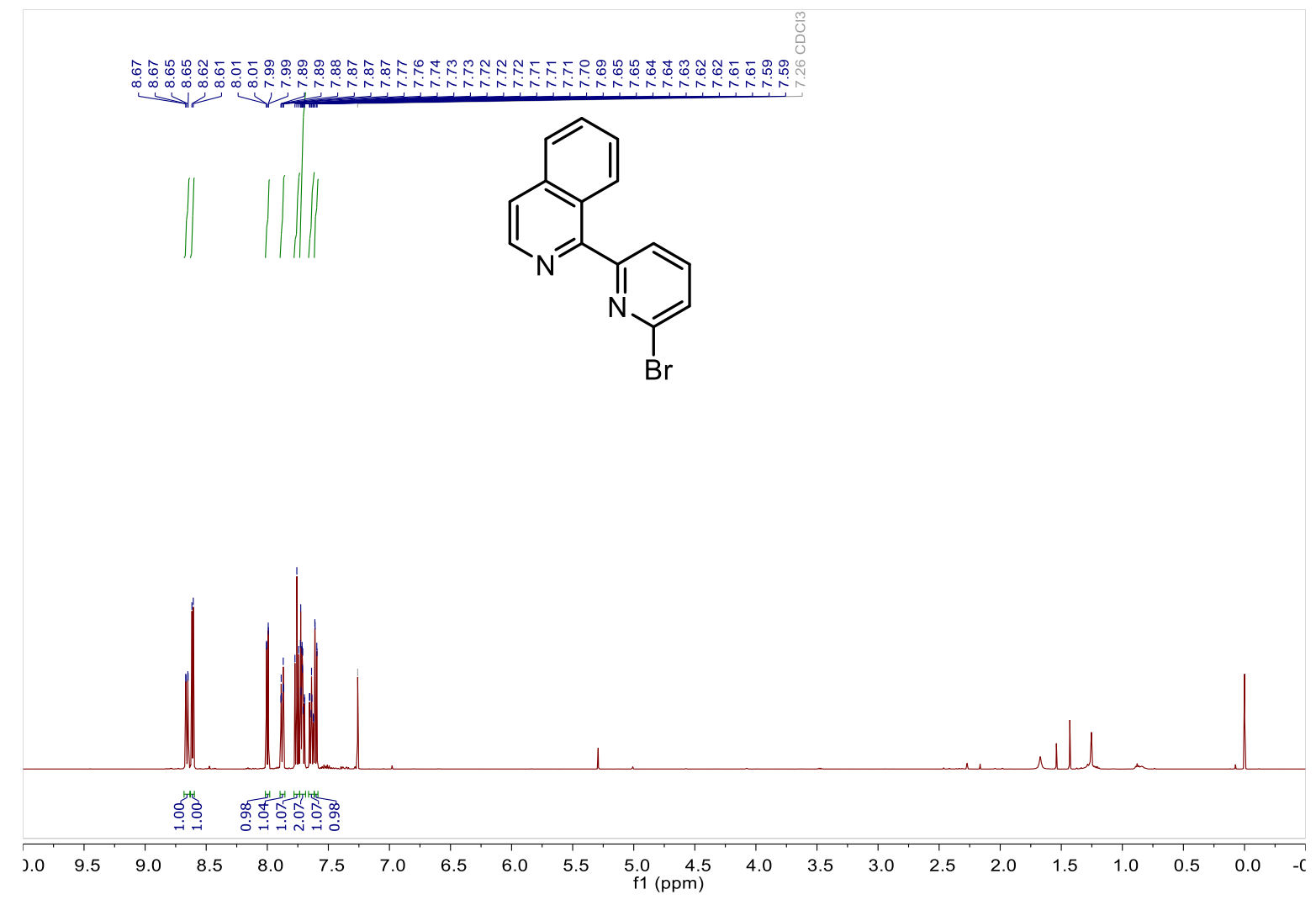

${ }^{13} \mathrm{C}$ NMR (126 MHz, $\left.\mathrm{CDCl}_{3}\right): 26$

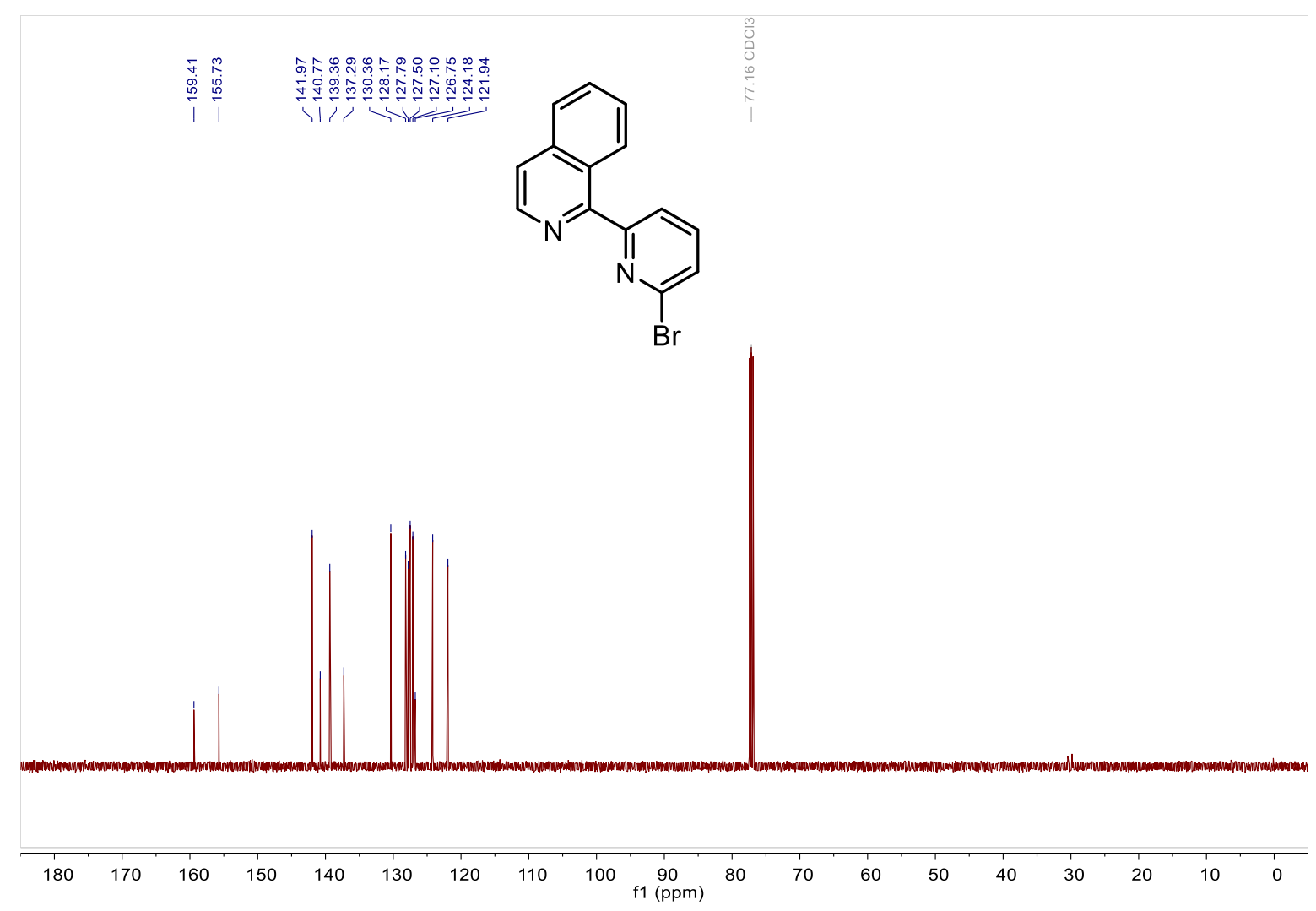


${ }^{1} \mathrm{H}$ NMR (500 MHz, $\mathrm{CDCl}_{3}$ ): 27

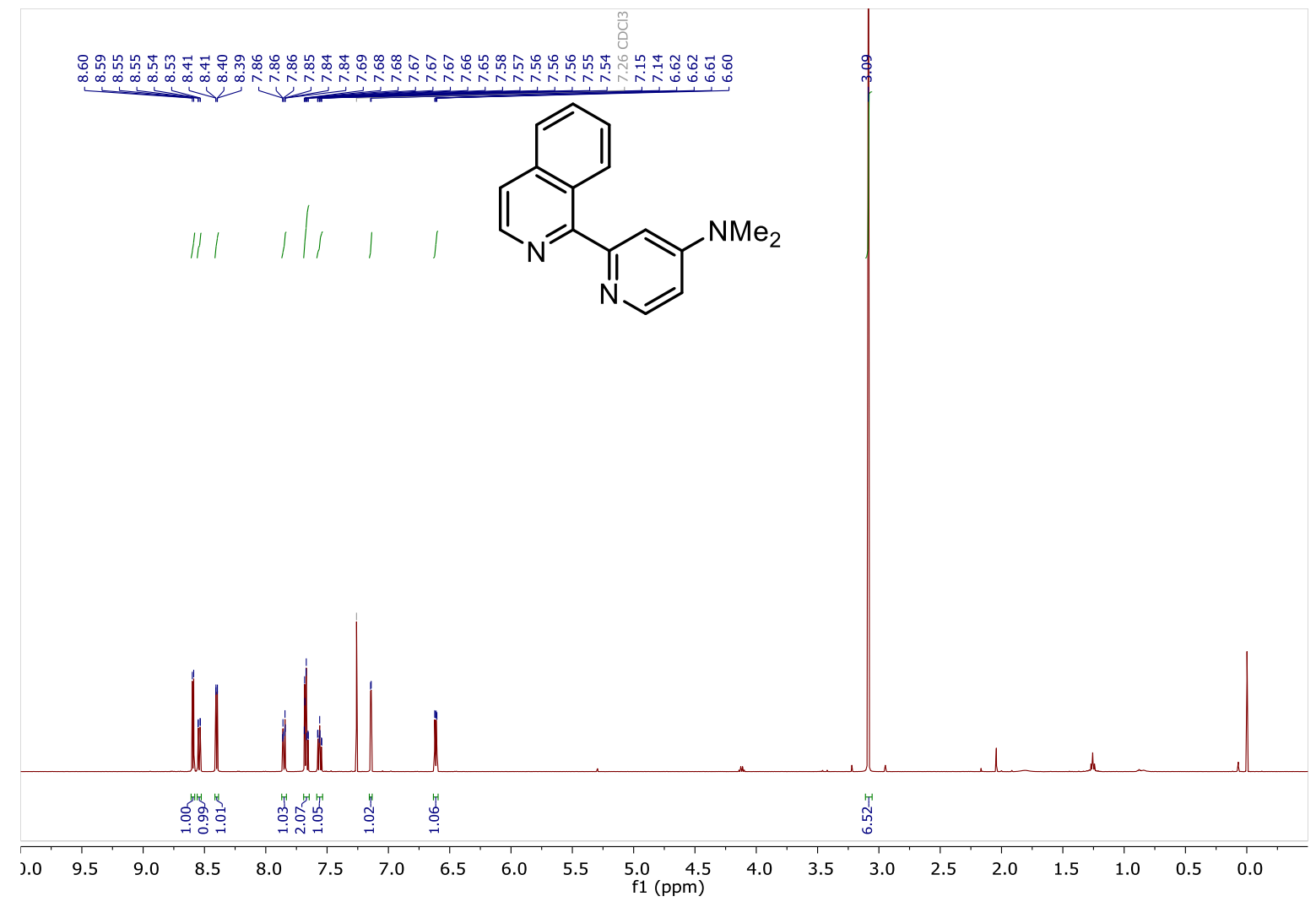

${ }^{13} \mathrm{C}$ NMR (126 MHz, $\mathrm{CDCl}_{3}$ ): 27

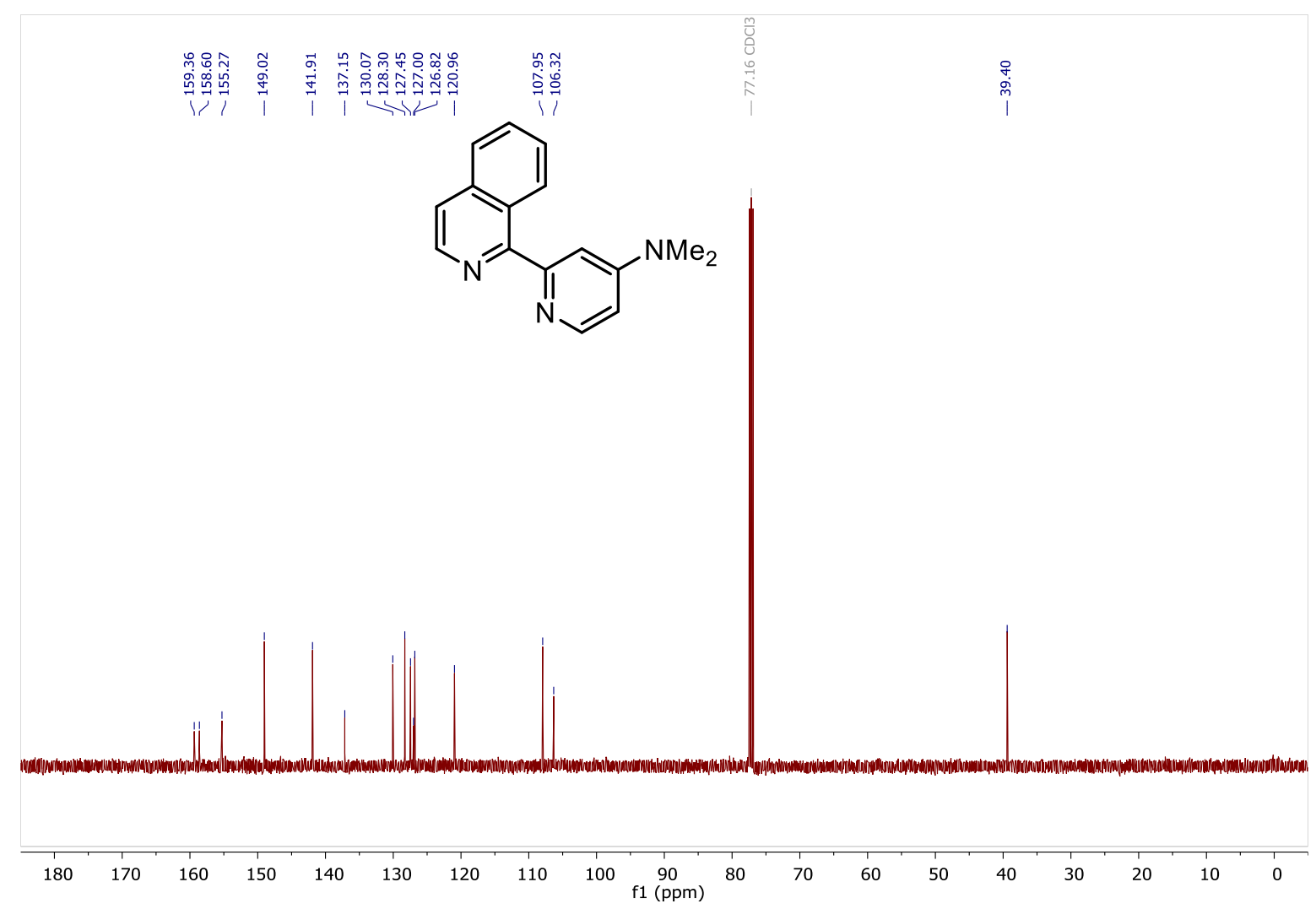


${ }^{1} \mathrm{H}$ NMR (400 MHz, $\mathrm{CDCl}_{3}$ ): 28

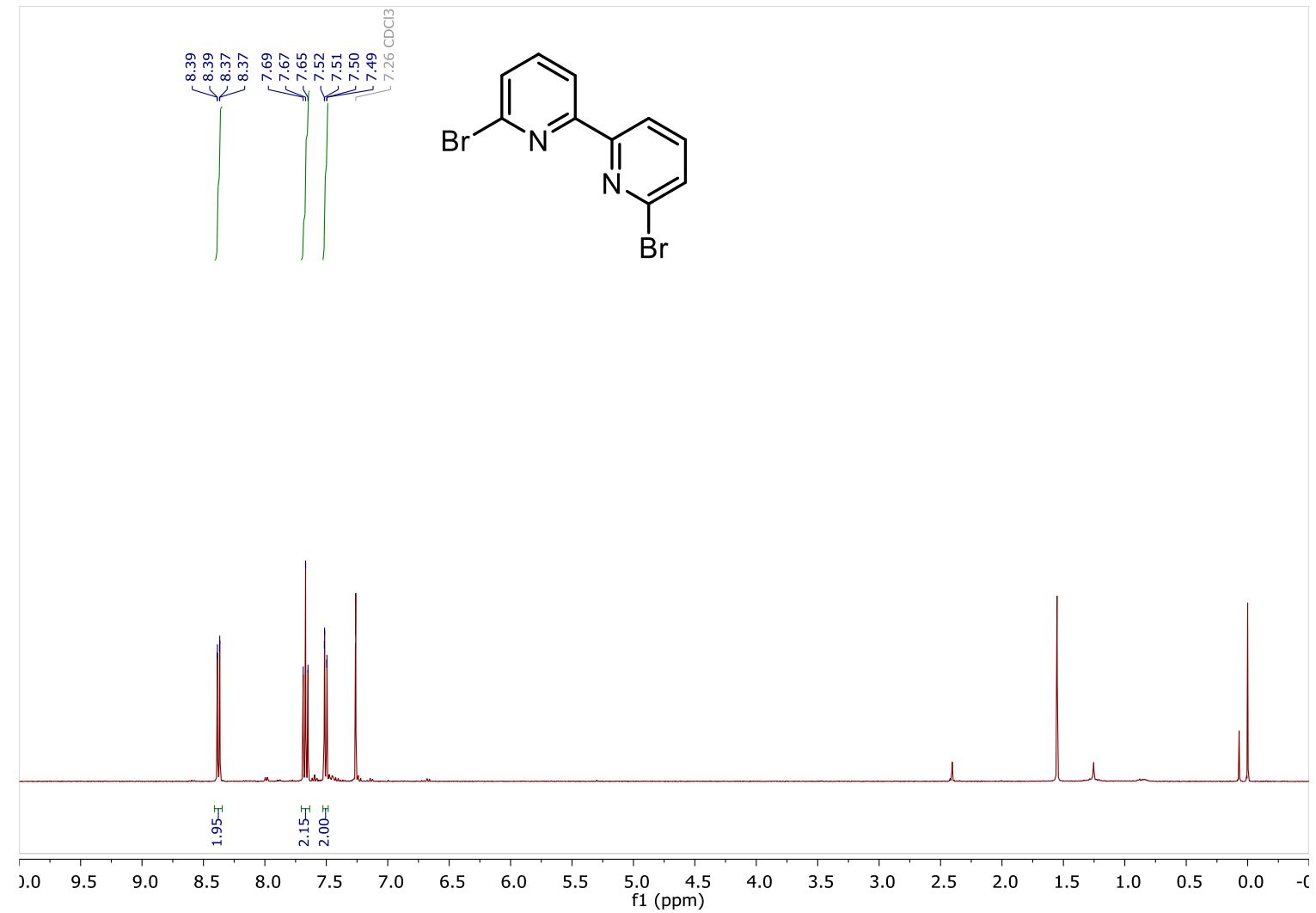

${ }^{13} \mathrm{C}$ NMR (101 MHz, $\left.\mathrm{CDCl}_{3}\right): 28$

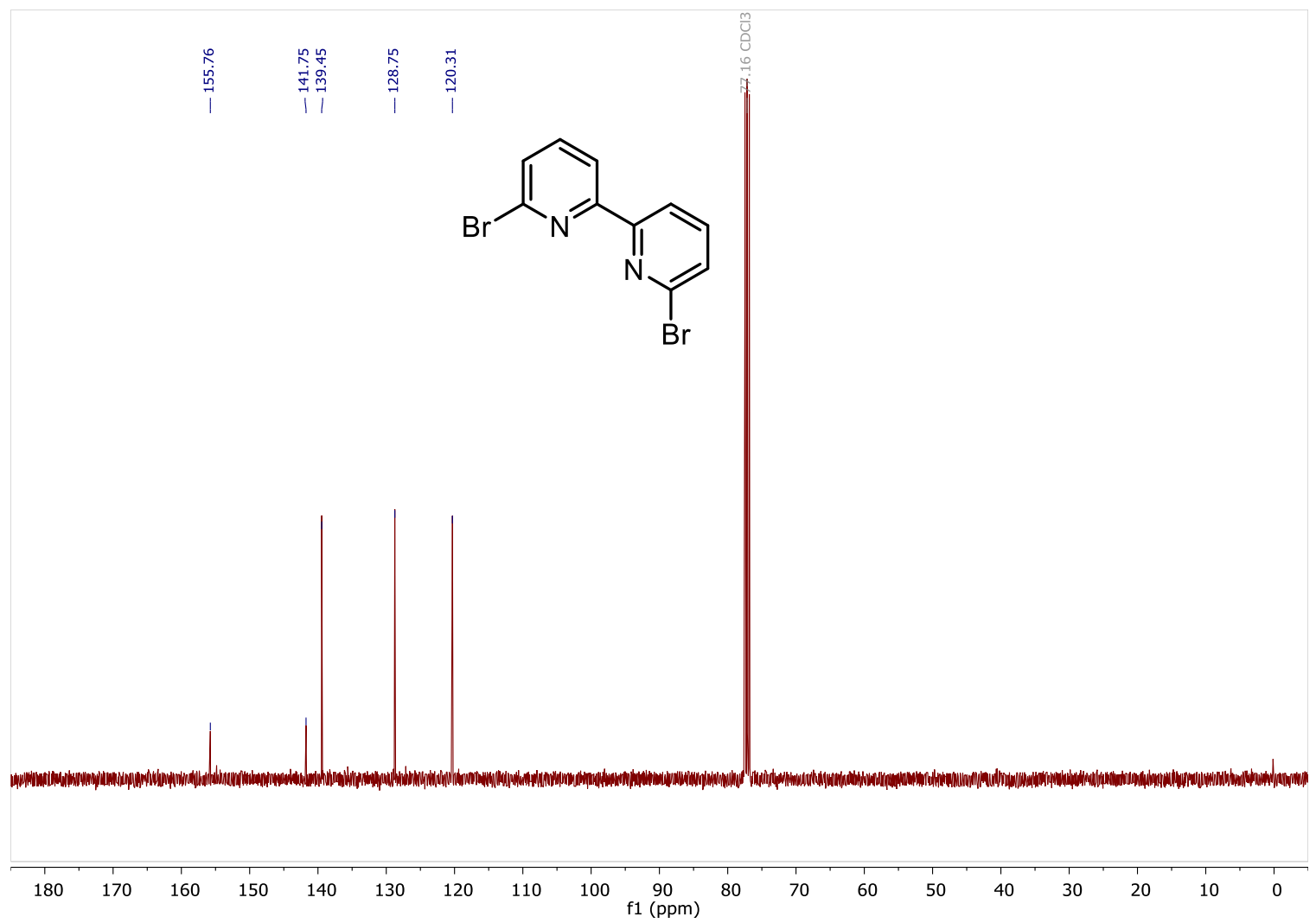


${ }^{1} \mathrm{H}$ NMR (500 MHz, $\mathrm{CDCl}_{3}$ ): 29

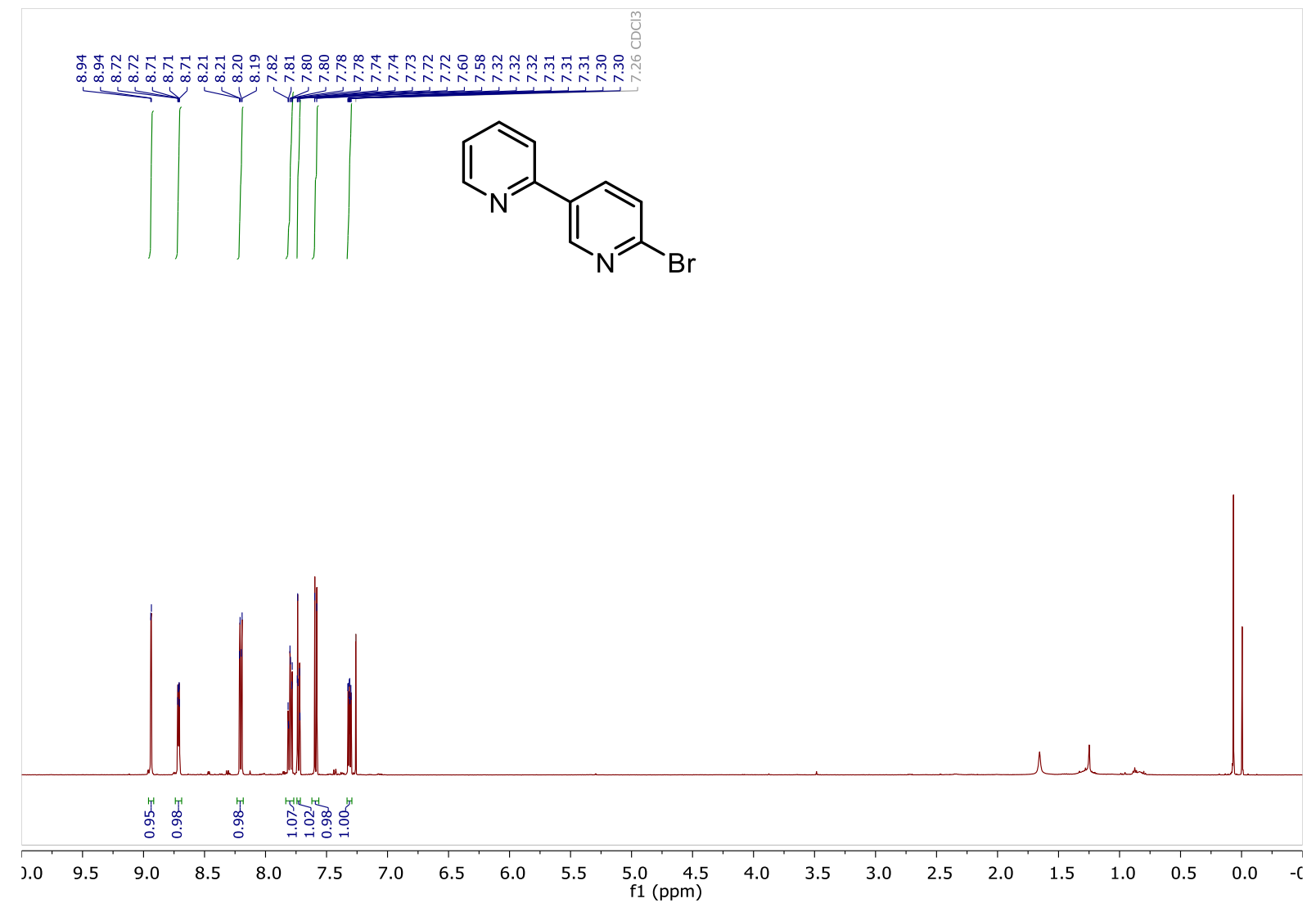

${ }^{13} \mathrm{C}$ NMR (126 MHz, $\mathrm{CDCl}_{3}$ ): 29

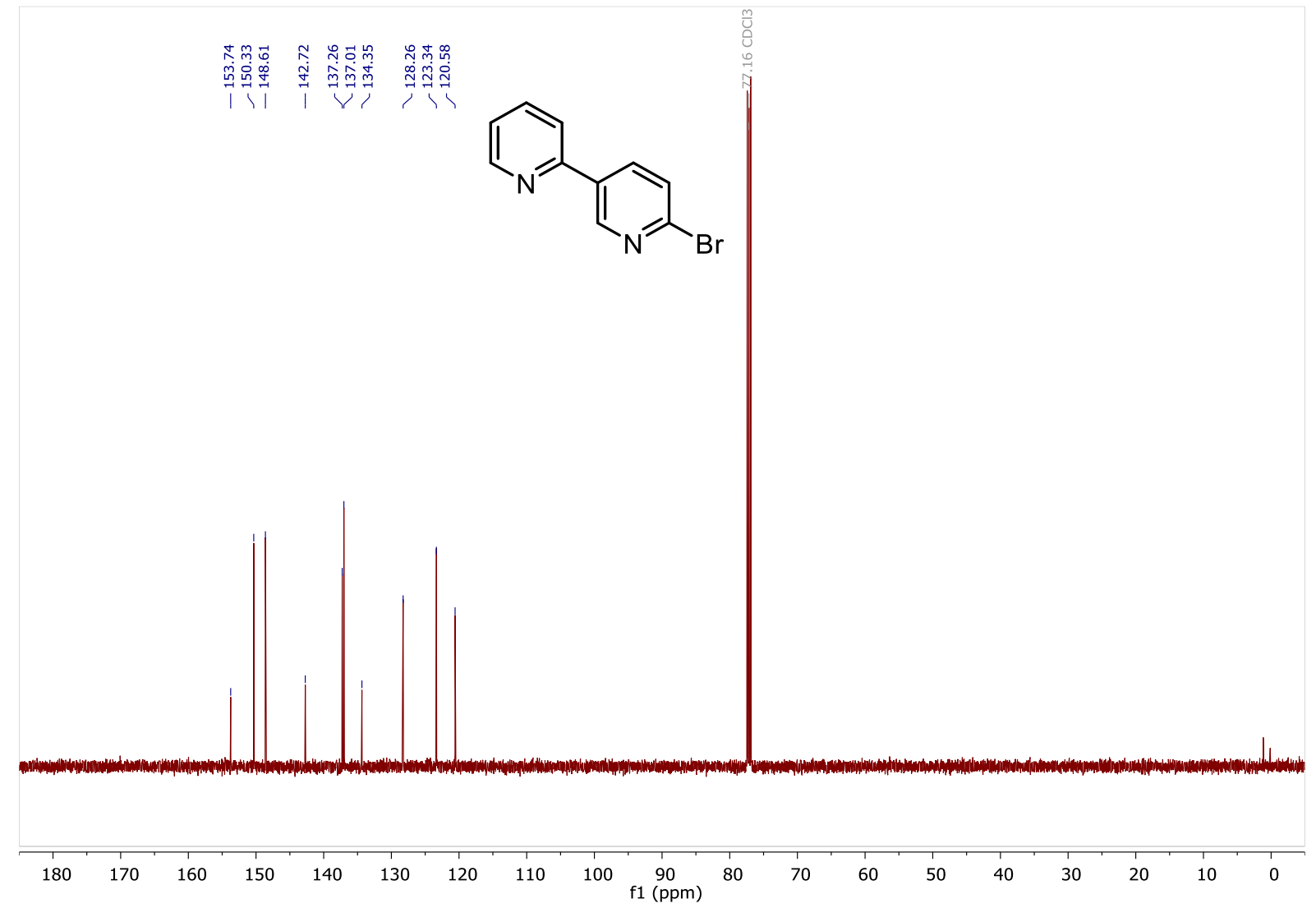


${ }^{1} \mathrm{H}$ NMR (500 MHz, $\mathrm{CDCl}_{3}$ ): 30

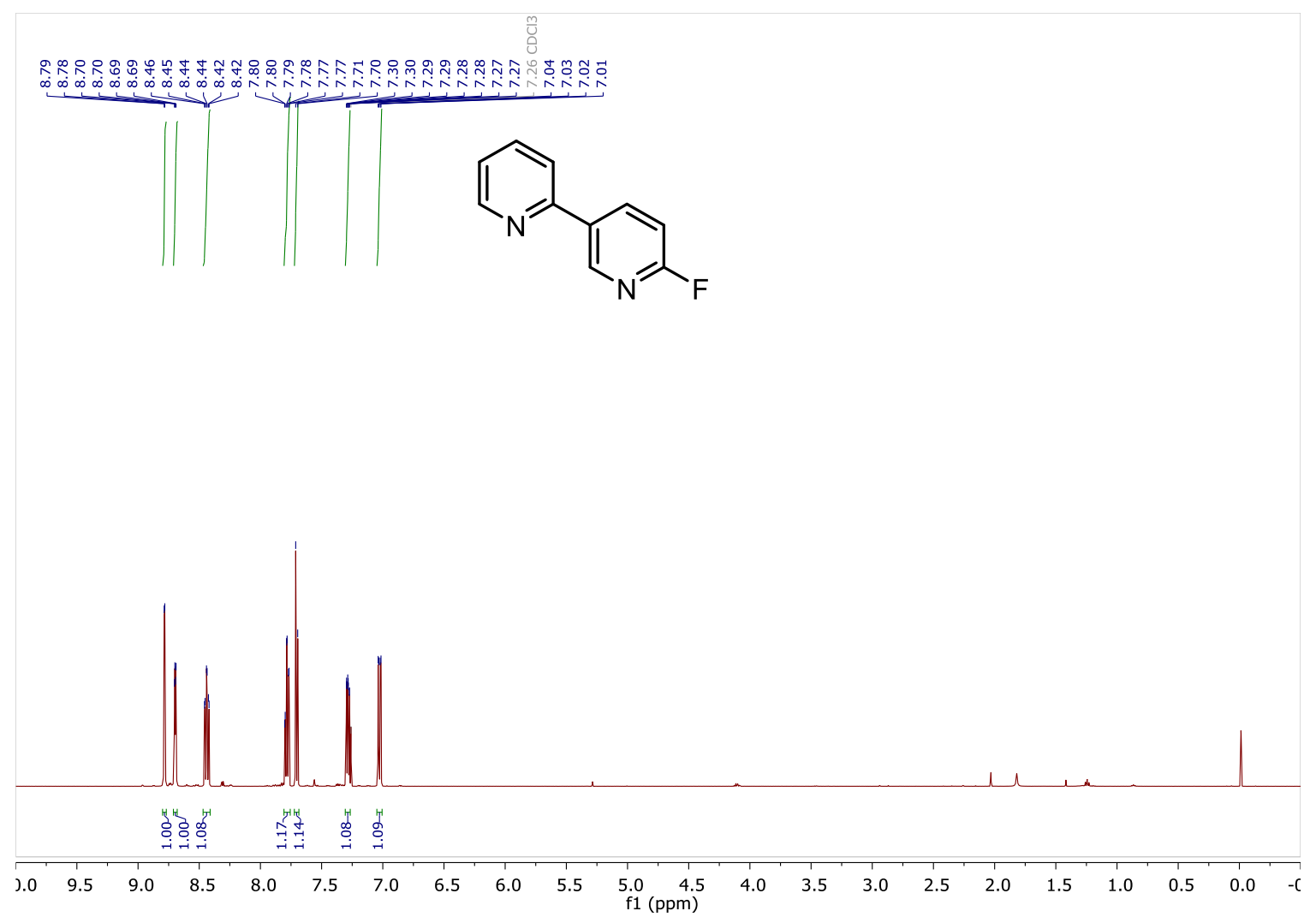

${ }^{13} \mathrm{C}$ NMR (126 MHz, $\left.\mathrm{CDCl}_{3}\right): 30$

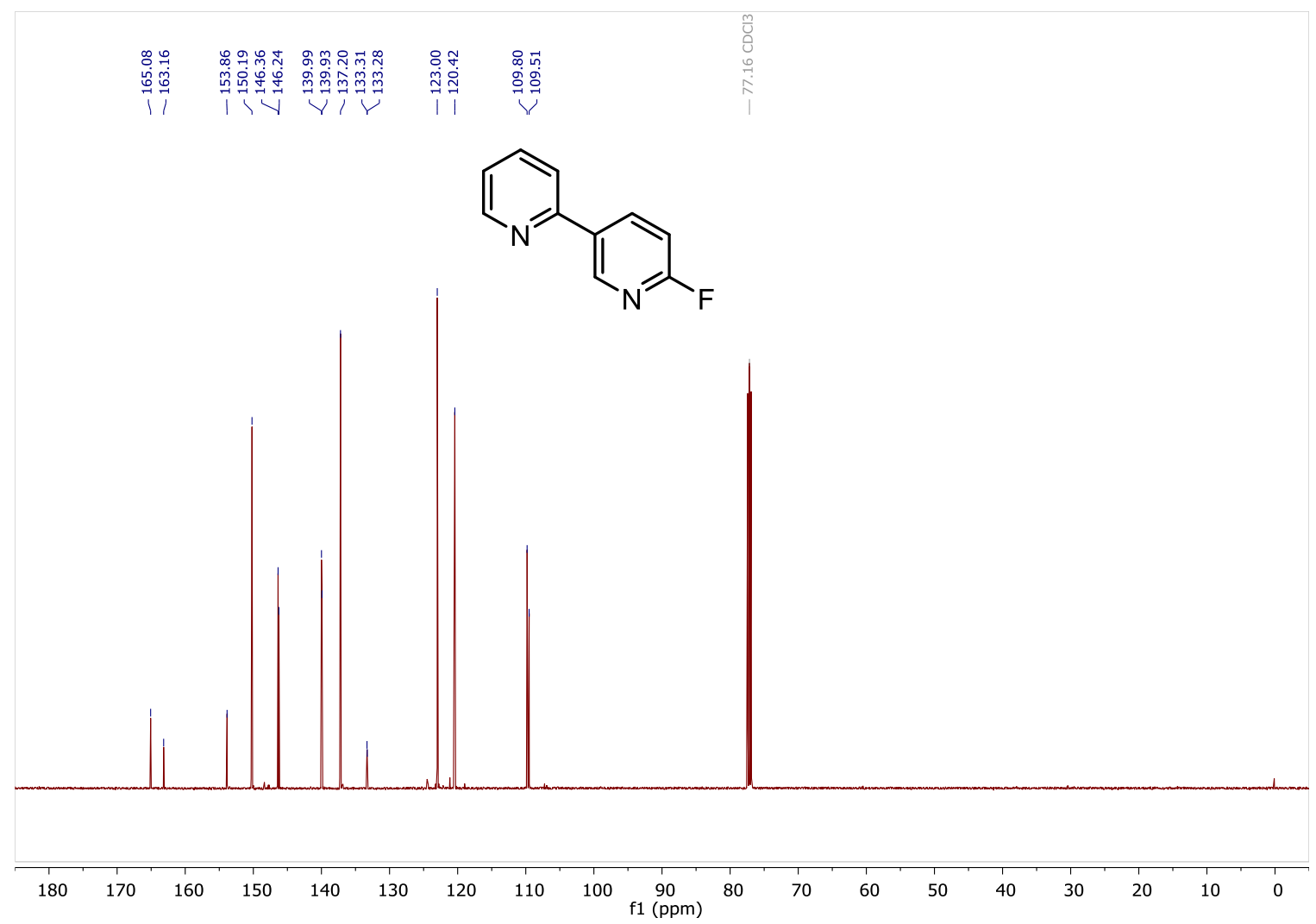

S84 
${ }^{1} \mathrm{H}$ NMR (400 MHz, $\left.\mathrm{CDCl}_{3}\right): 31$

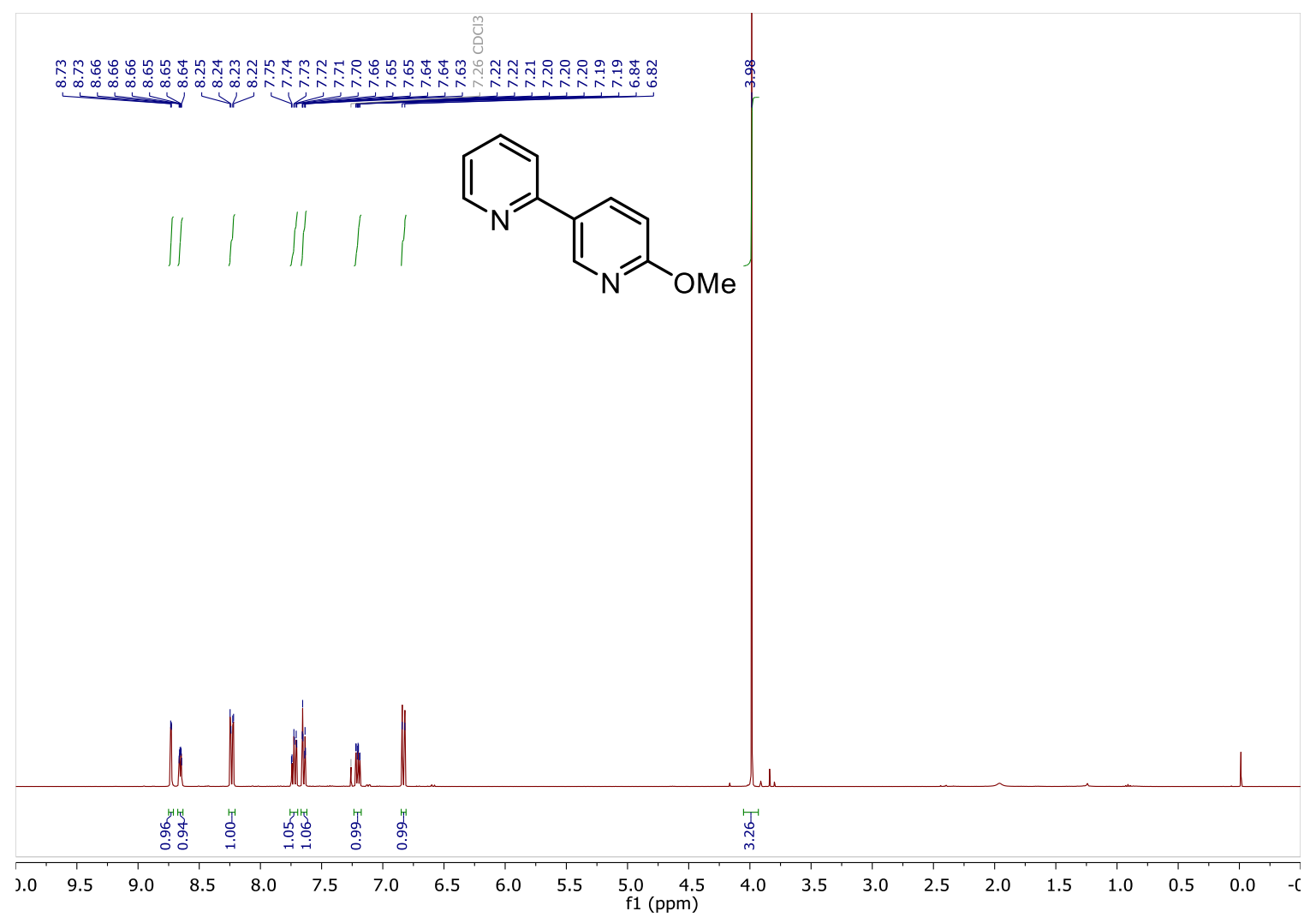

${ }^{13} \mathrm{C}$ NMR (101 MHz, $\left.\mathrm{CDCl}_{3}\right): 31$

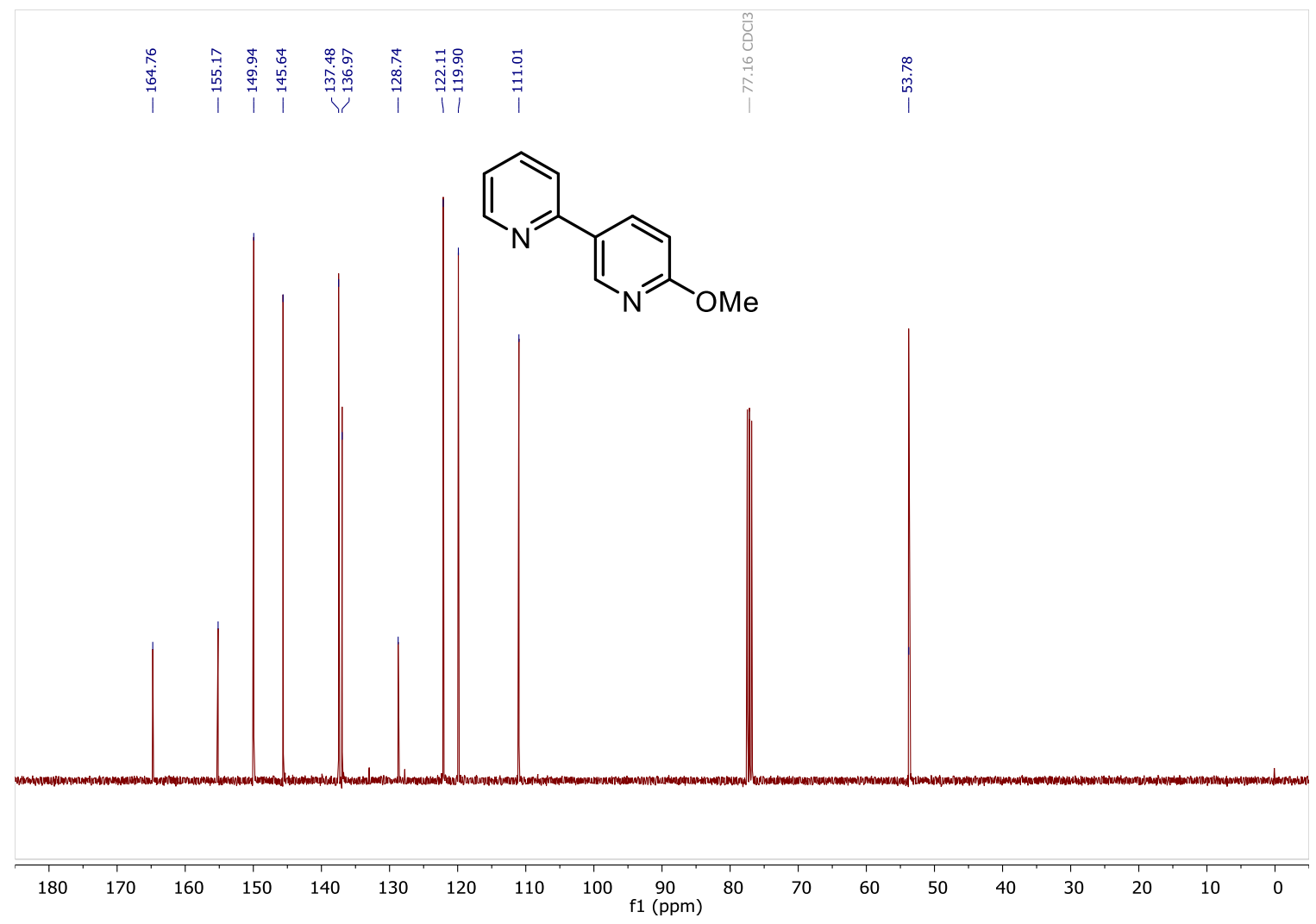

S85 
${ }^{1} \mathrm{H}$ NMR (500 MHz, $\mathrm{CDCl}_{3}$ ): 32

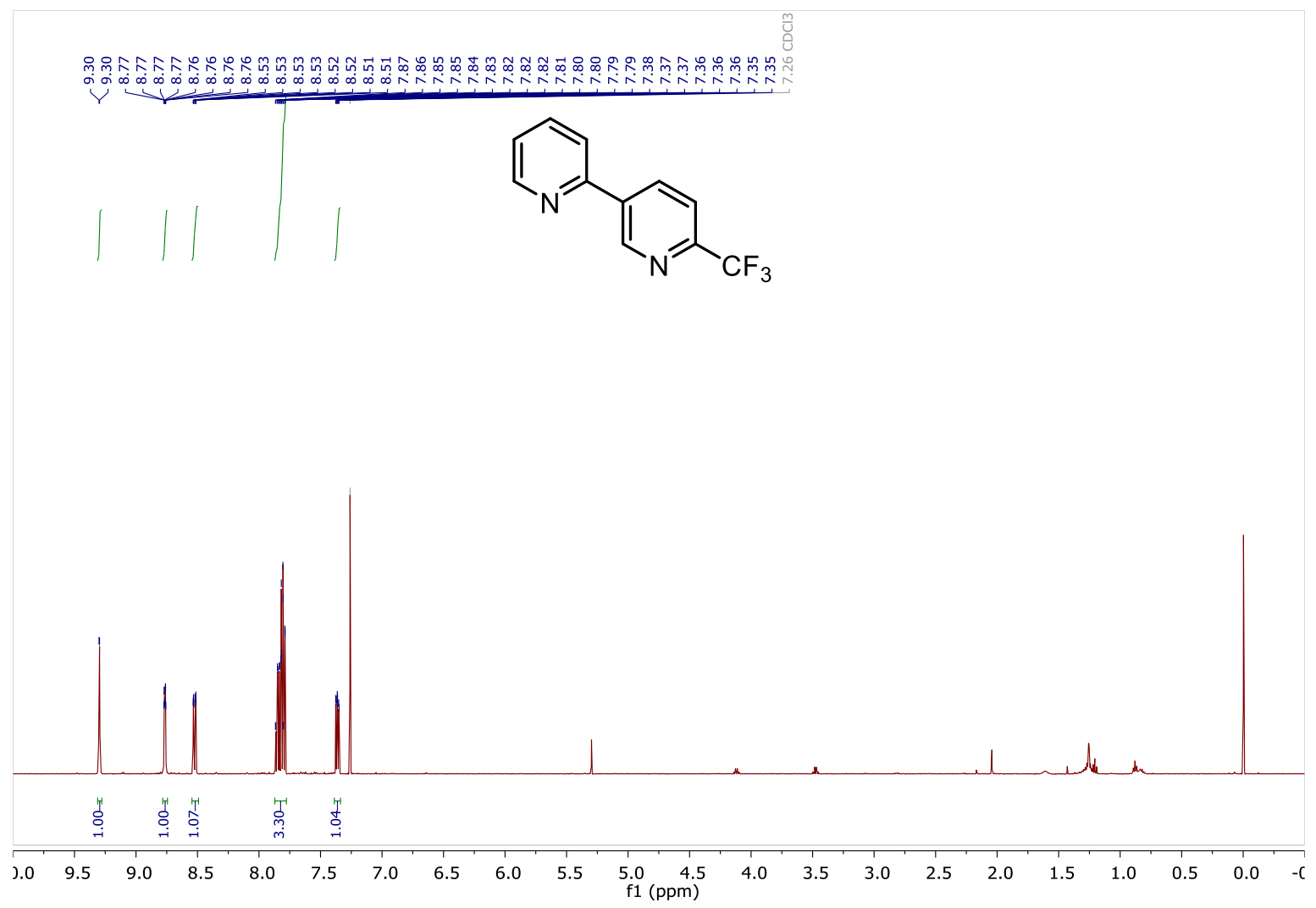

${ }^{13} \mathrm{C}$ NMR (101 MHz, $\left.\mathrm{CDCl}_{3}\right): 32$

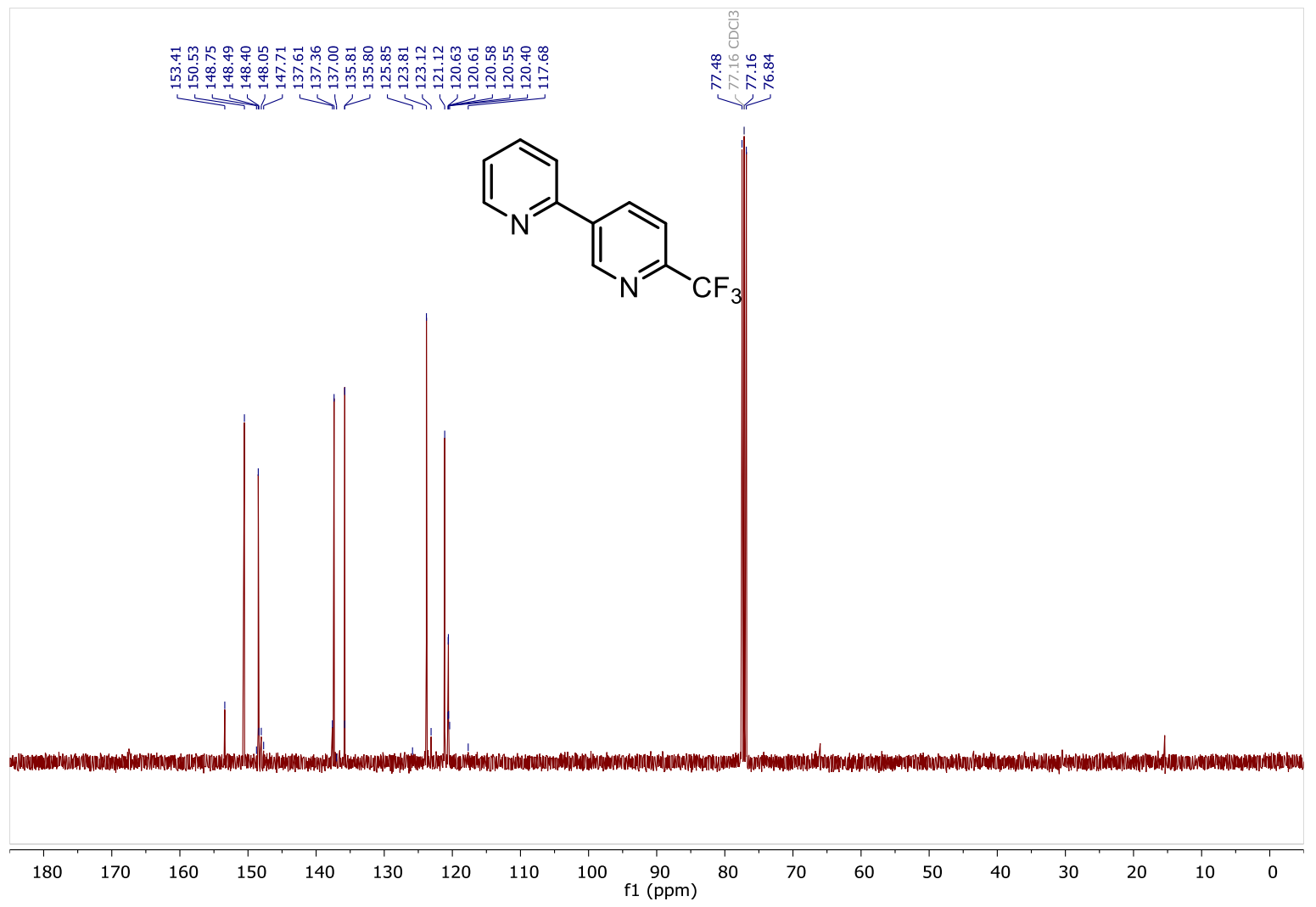

S86 
${ }^{1} \mathrm{H}$ NMR (400 MHz, $\mathrm{CDCl}_{3}$ ): 33

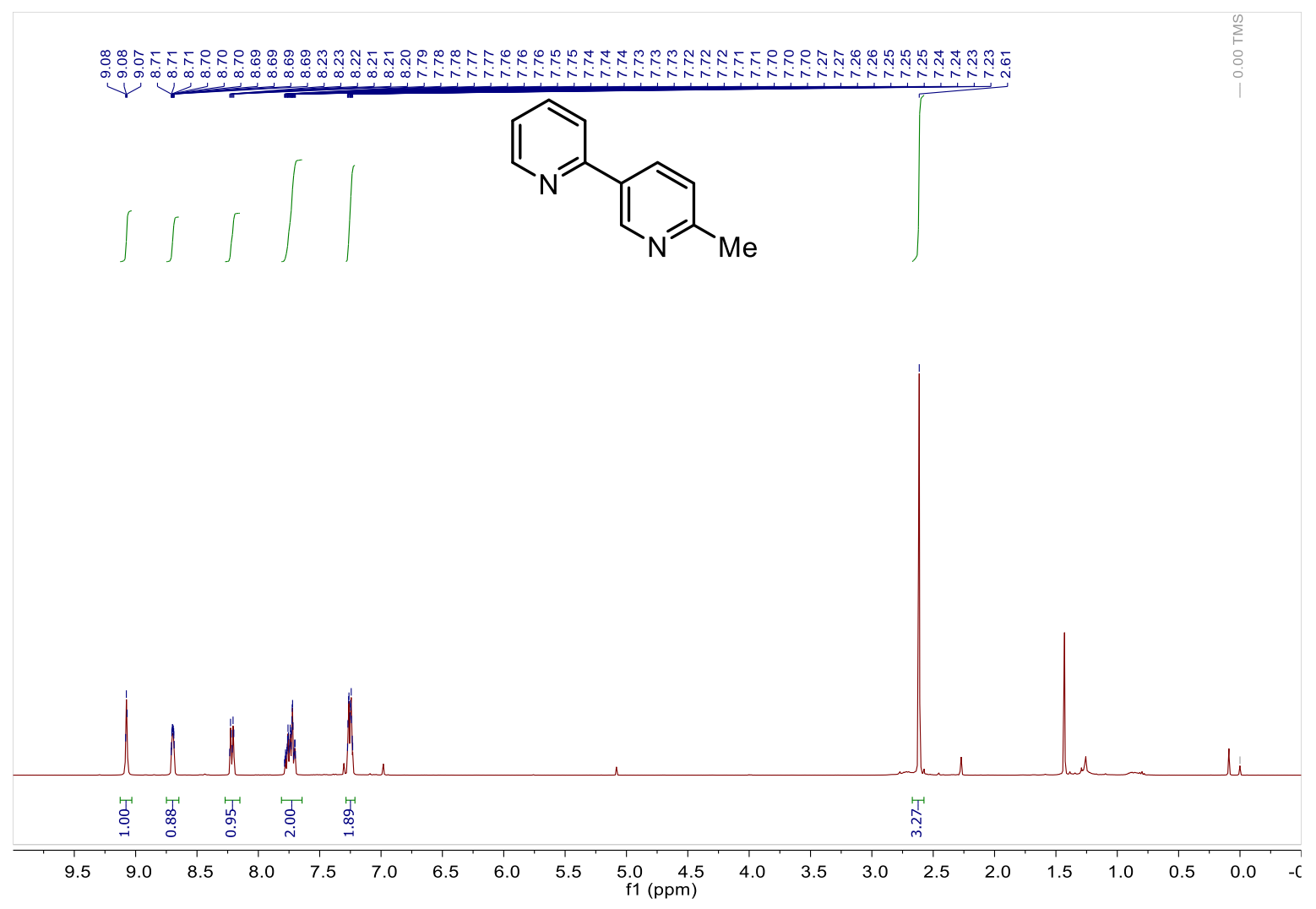

${ }^{13} \mathrm{C}$ NMR (101 MHz, $\left.\mathrm{CDCl}_{3}\right): 33$

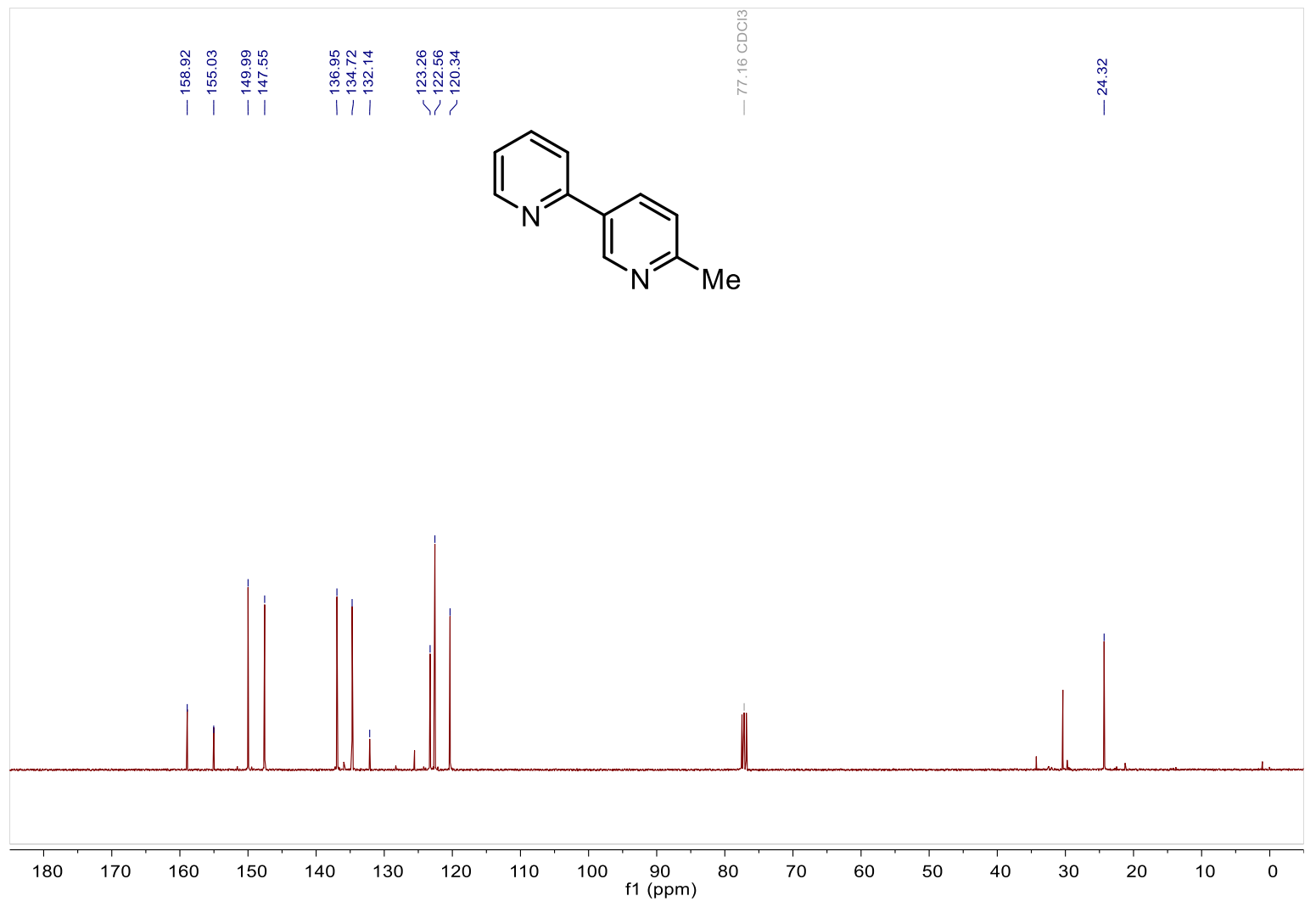

S87 
${ }^{1} \mathrm{H}$ NMR (500 MHz, $\mathrm{CDCl}_{3}$ ): 34

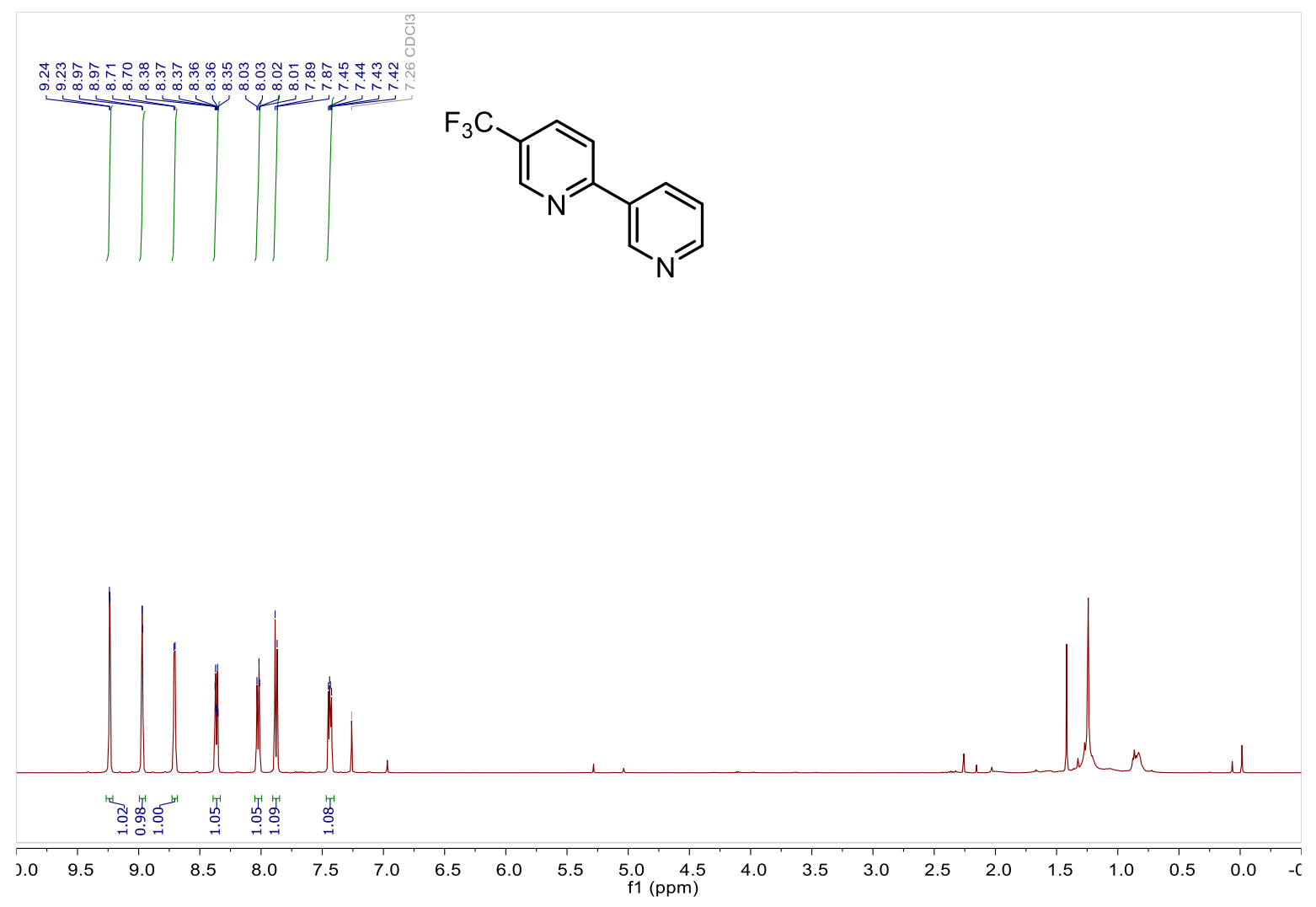

${ }^{13} \mathrm{C}$ NMR (126 MHz, $\left.\mathrm{CDCl}_{3}\right): 34$

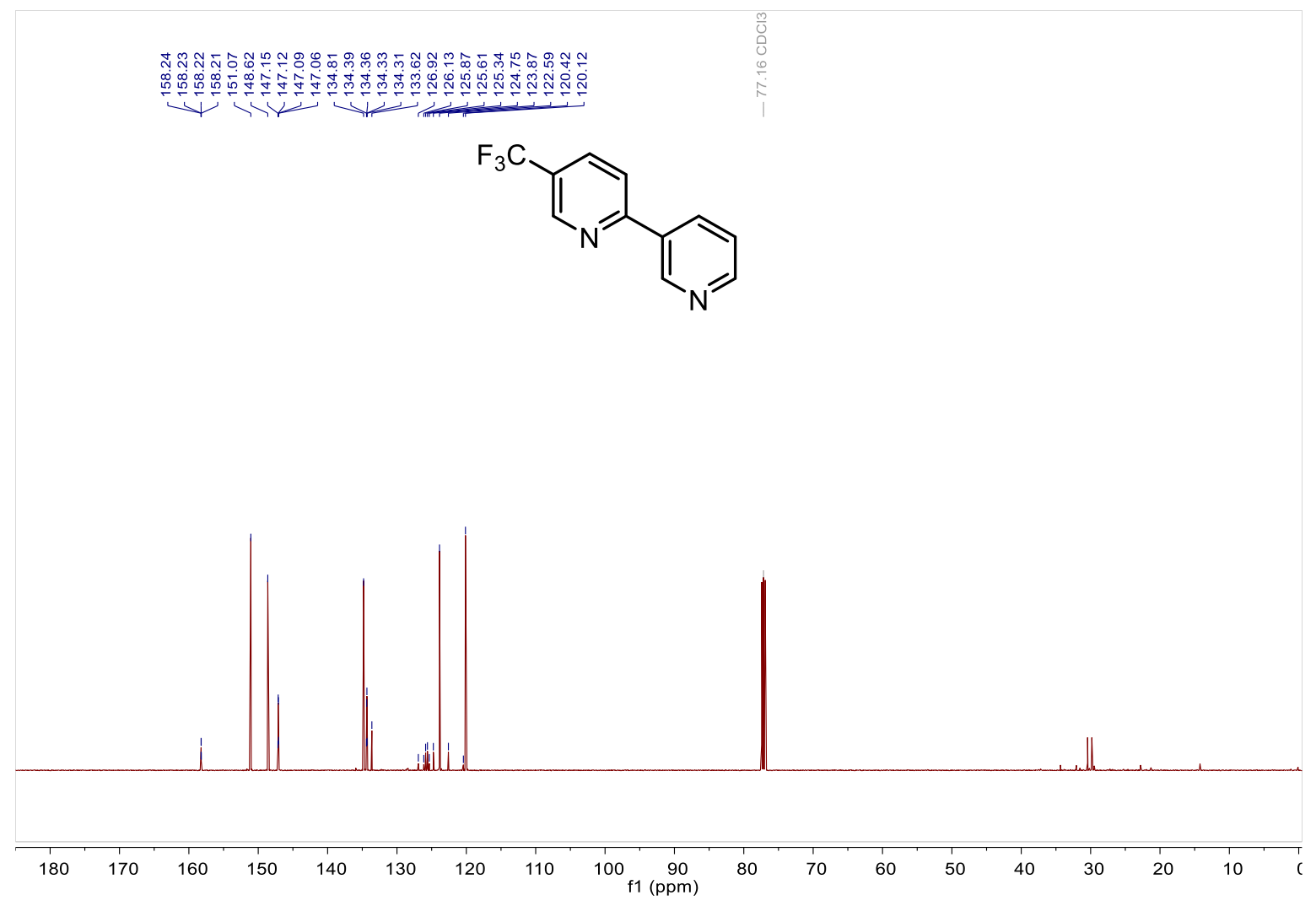

S88 
${ }^{1} \mathrm{H}$ NMR (500 MHz, $\left.\mathrm{CDCl}_{3}\right): 35$

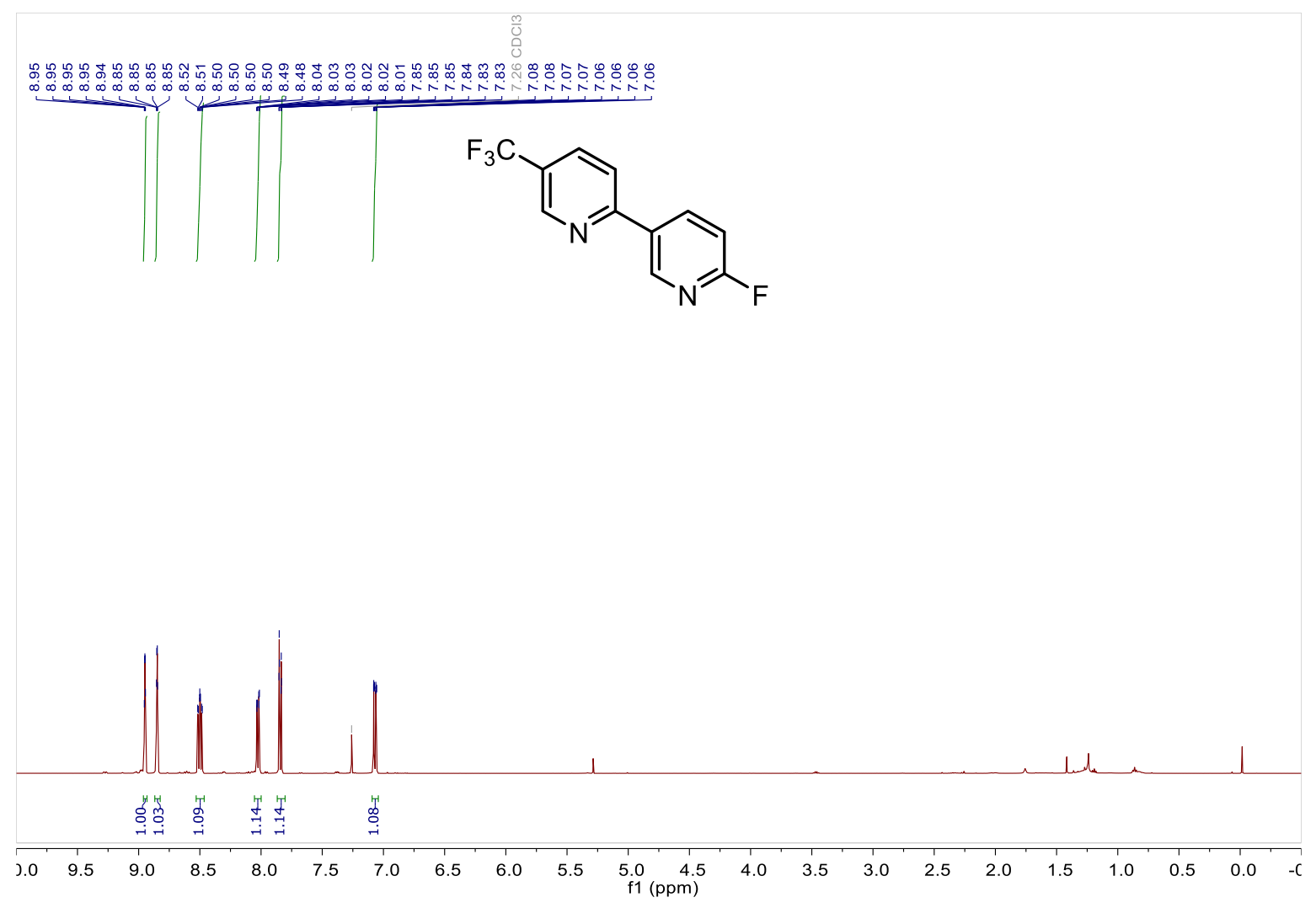

${ }^{13} \mathrm{C}$ NMR (126 MHz, $\left.\mathrm{CDCl}_{3}\right): 35$

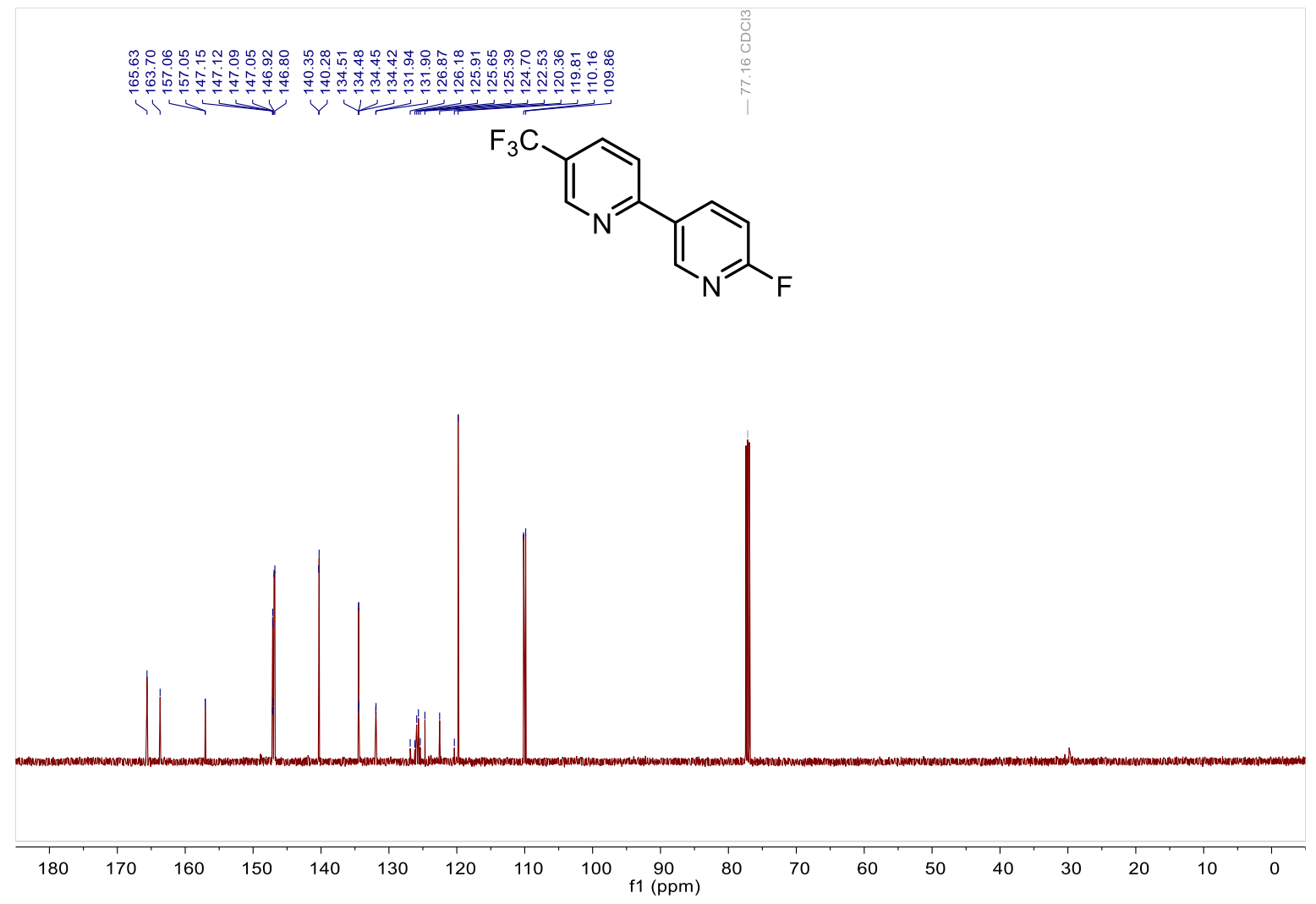

S89 
${ }^{1} \mathrm{H}$ NMR (500 MHz, $\left.\mathrm{CDCl}_{3}\right): 36$

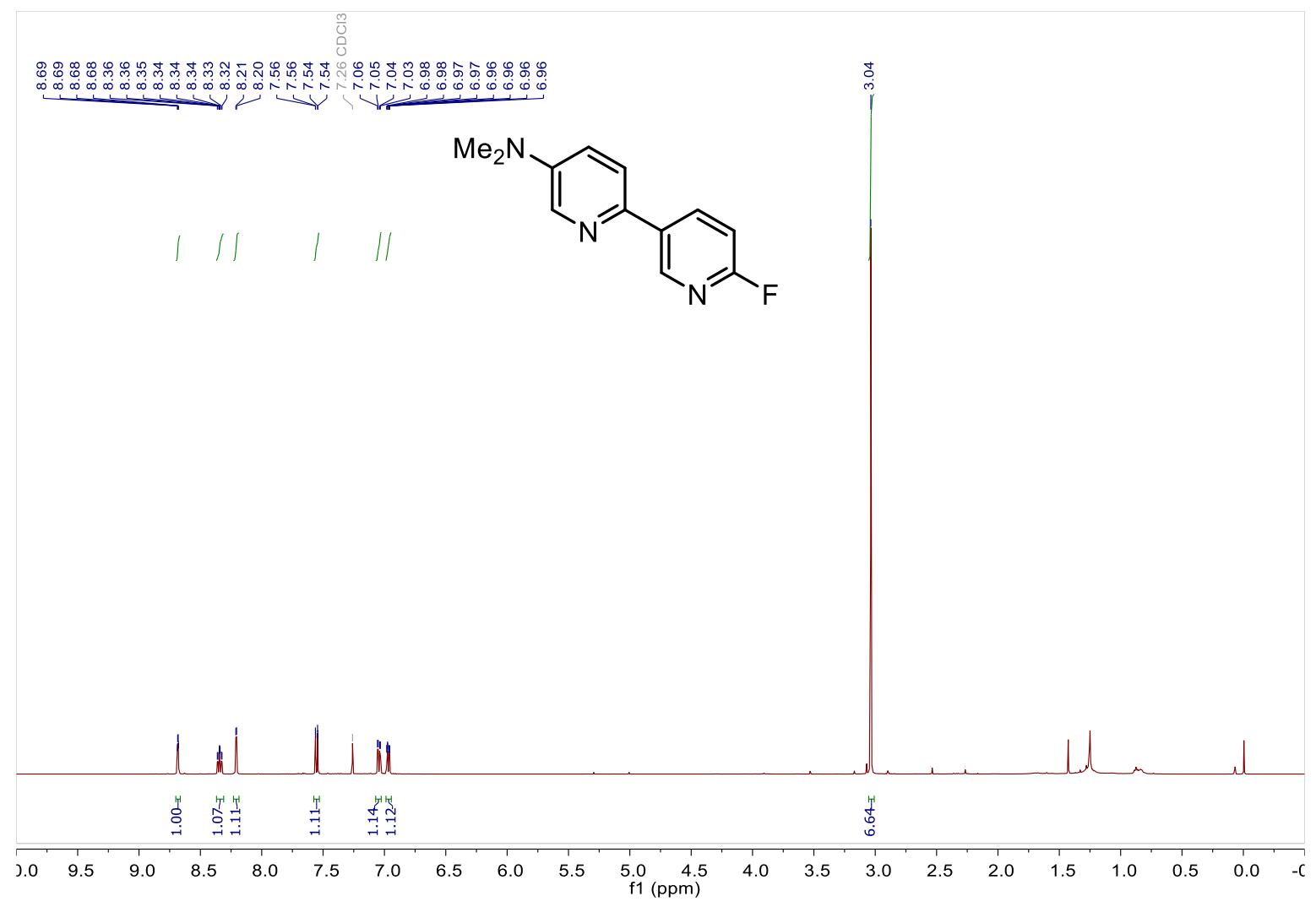

${ }^{13} \mathrm{C}$ NMR (126 MHz, $\left.\mathrm{CDCl}_{3}\right): 36$

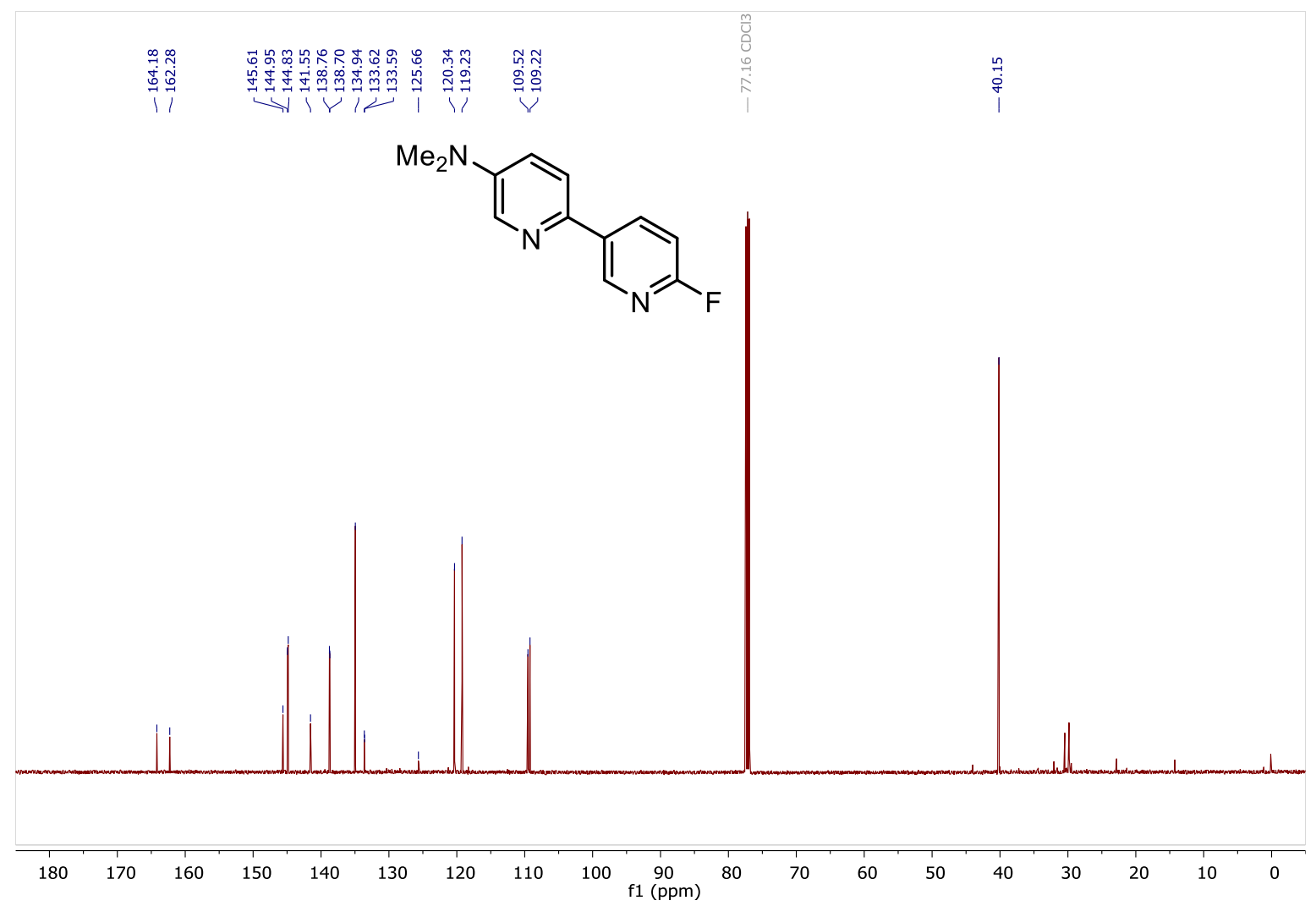

S90 
${ }^{1} \mathrm{H}$ NMR (400 MHz, $\mathrm{CDCl}_{3}$ ): 37

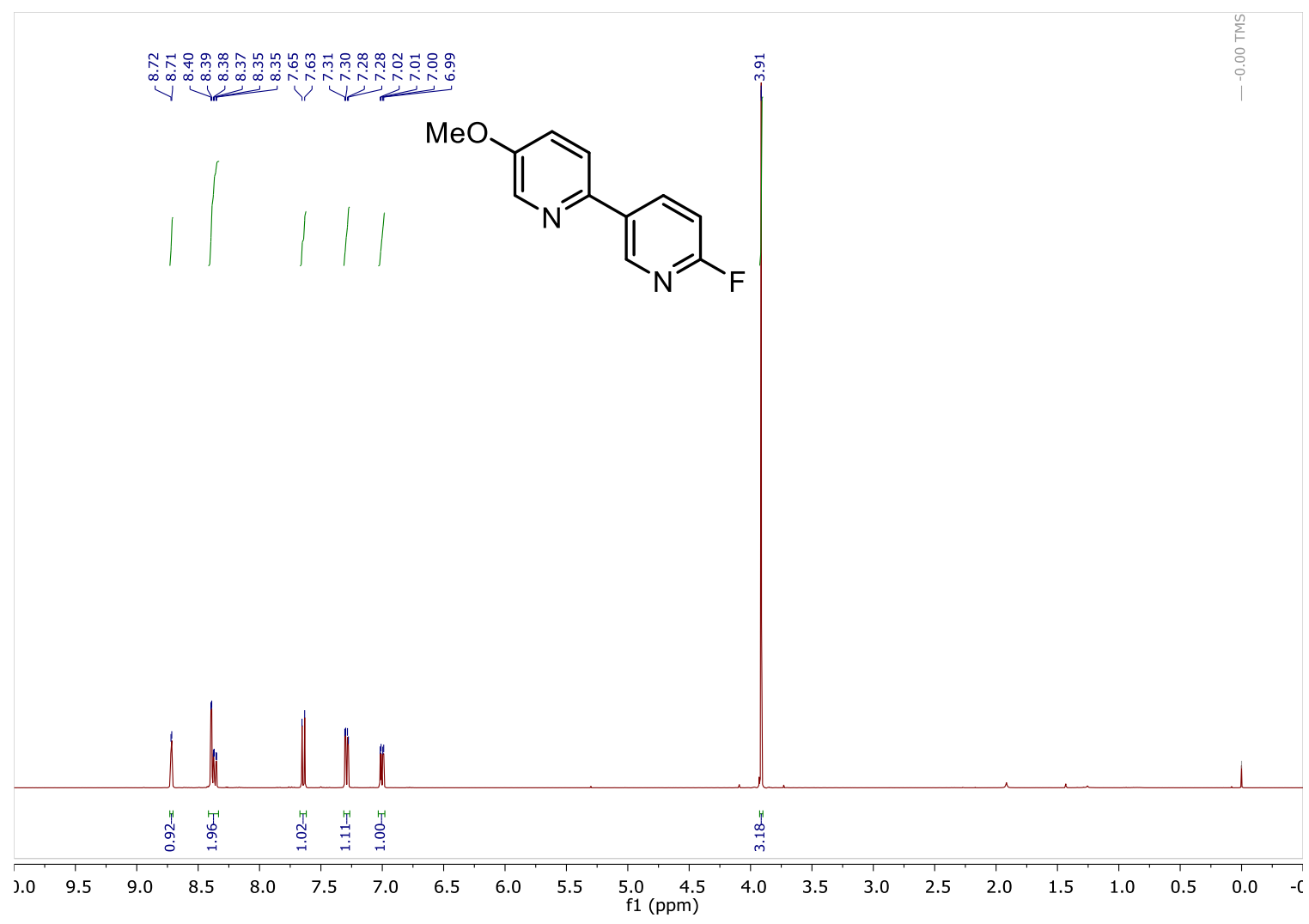

${ }^{13} \mathrm{C}$ NMR (101 MHz, $\left.\mathrm{CDCl}_{3}\right): 37$

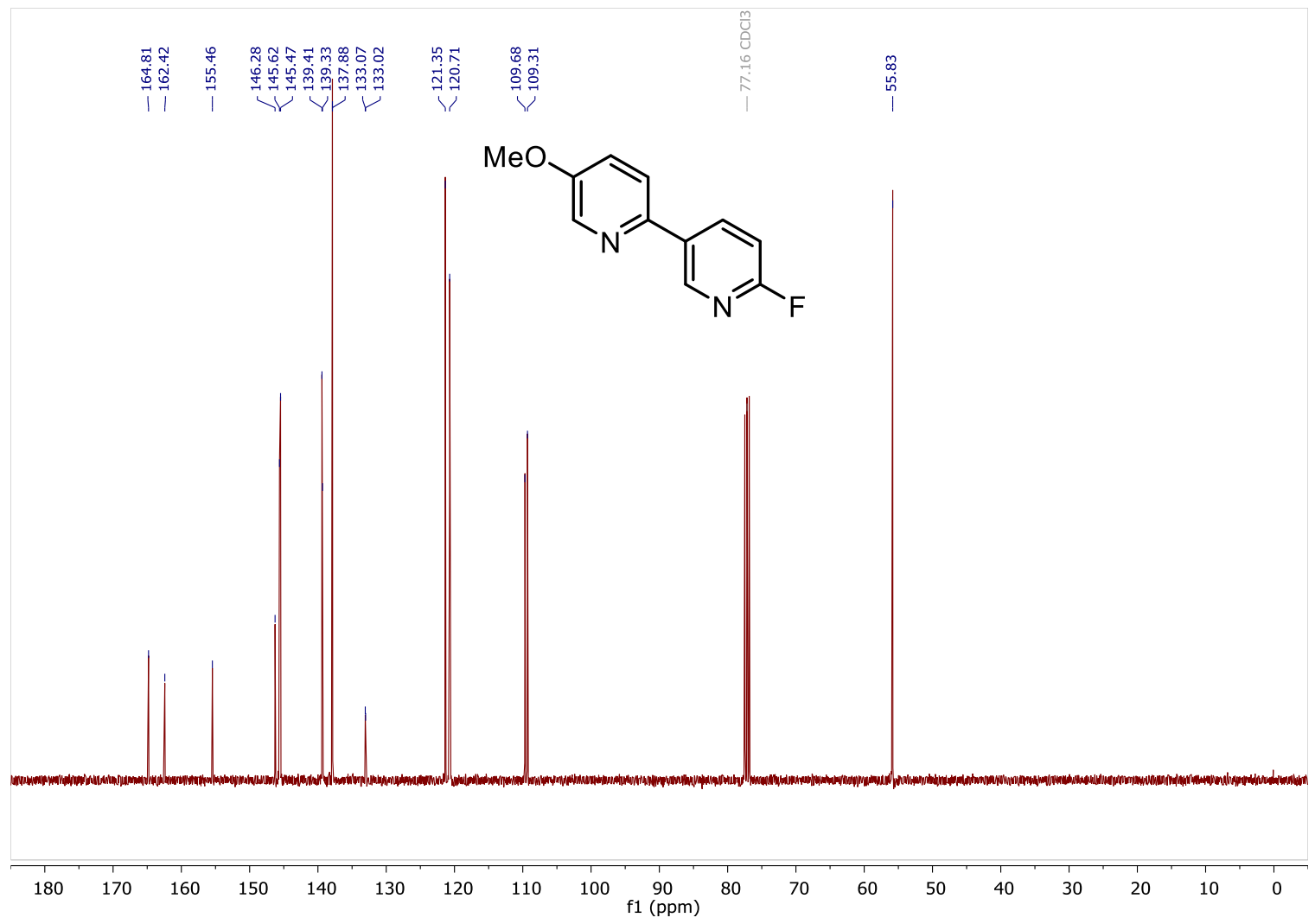

S91 
${ }^{1} \mathrm{H}$ NMR (400 MHz, $\mathrm{CDCl}_{3}$ ): 38

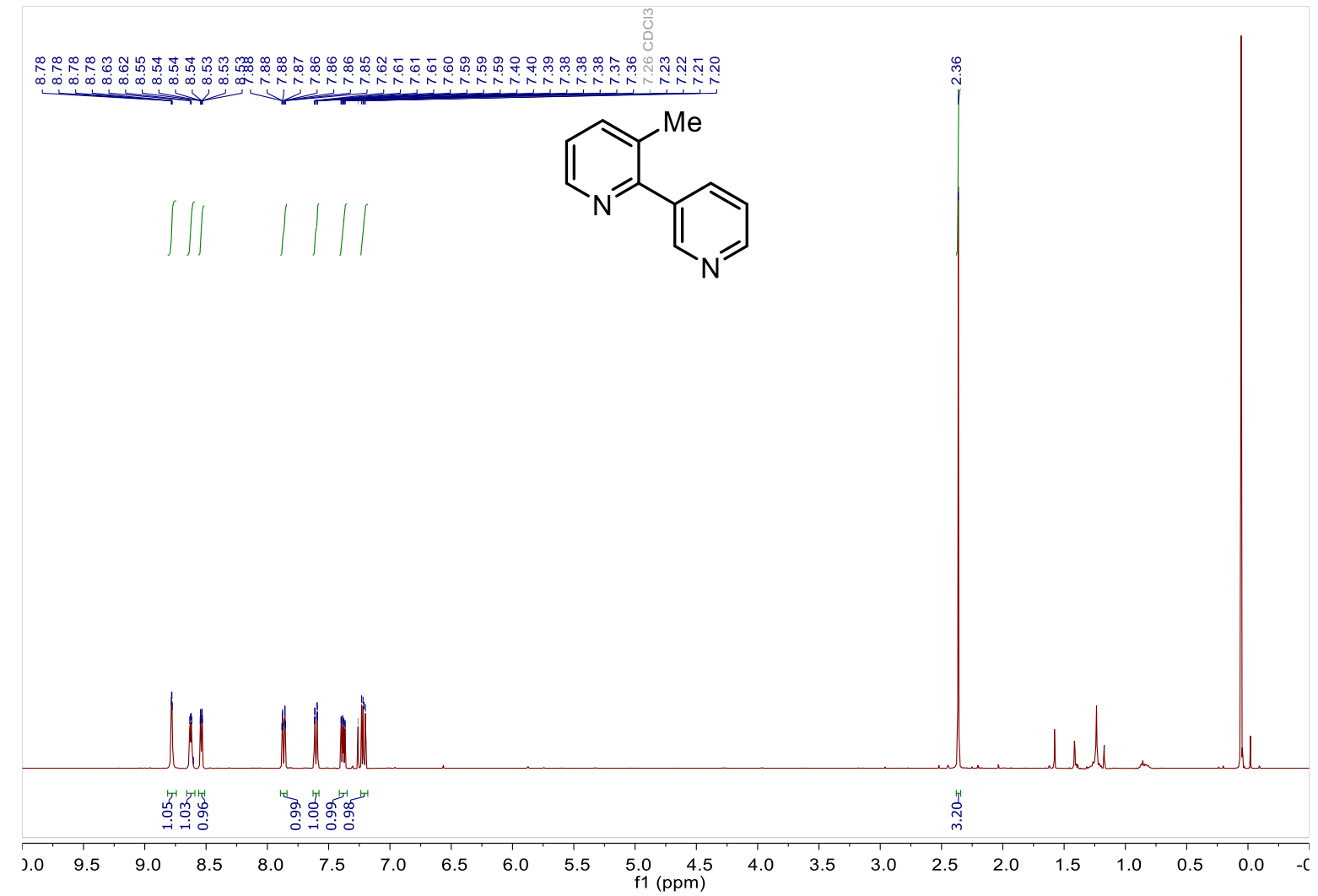

${ }^{13} \mathrm{C}$ NMR (101 MHz, $\left.\mathrm{CDCl}_{3}\right): 38$

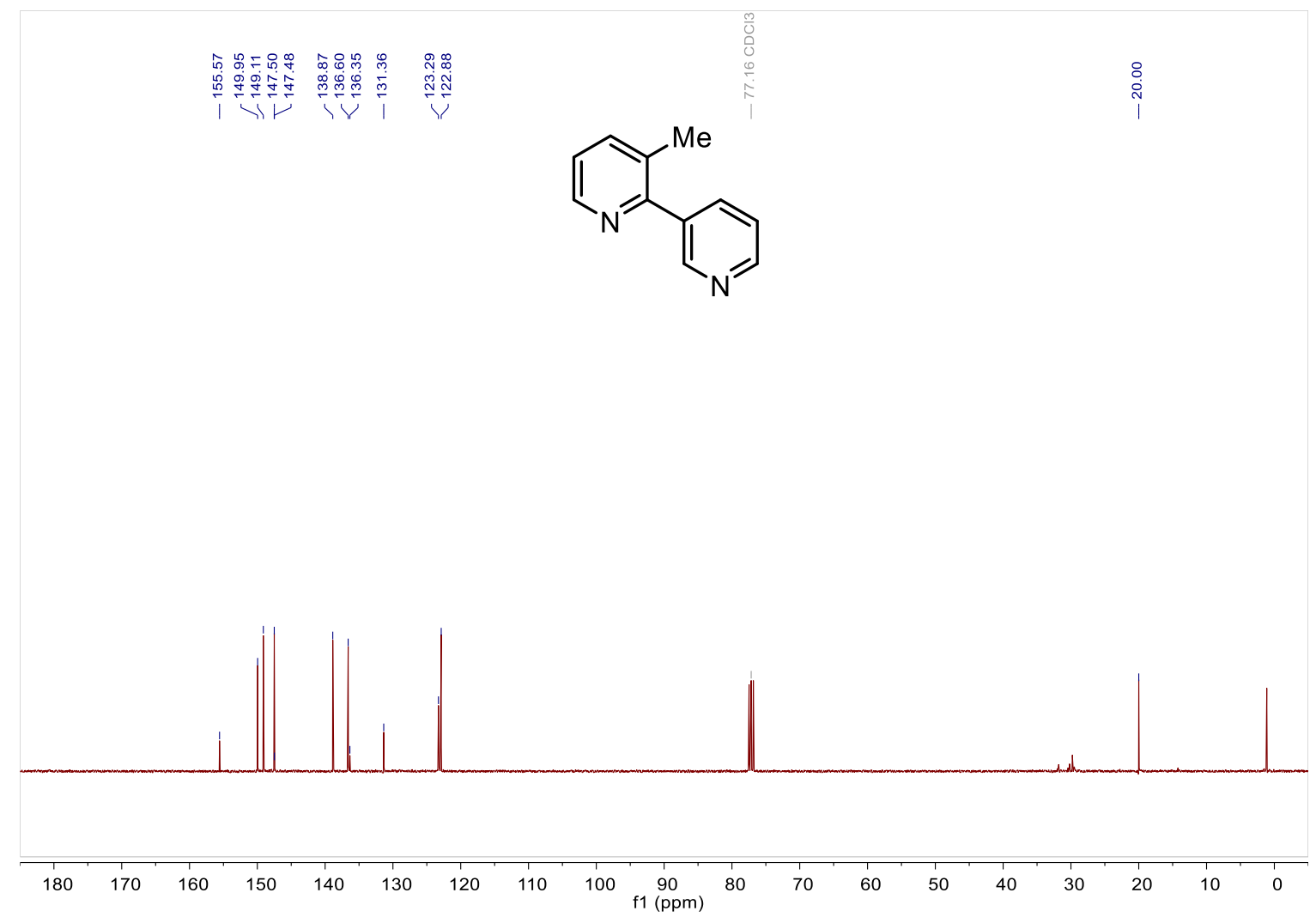


${ }^{1} \mathrm{H}$ NMR (400 MHz, $\mathrm{CDCl}_{3}$ ): 39

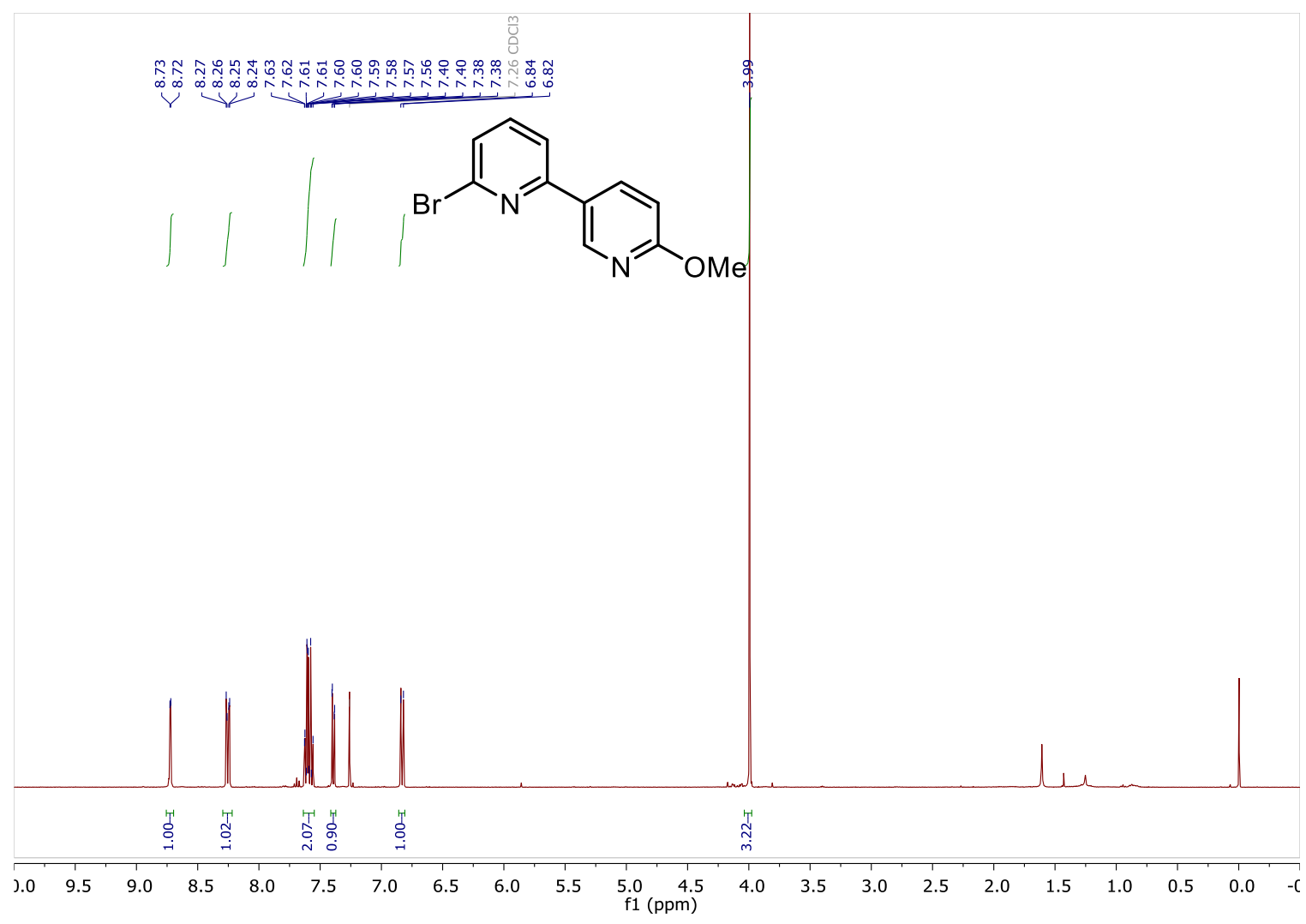

${ }^{13} \mathrm{C}$ NMR (101 MHz, $\left.\mathrm{CDCl}_{3}\right): 39$

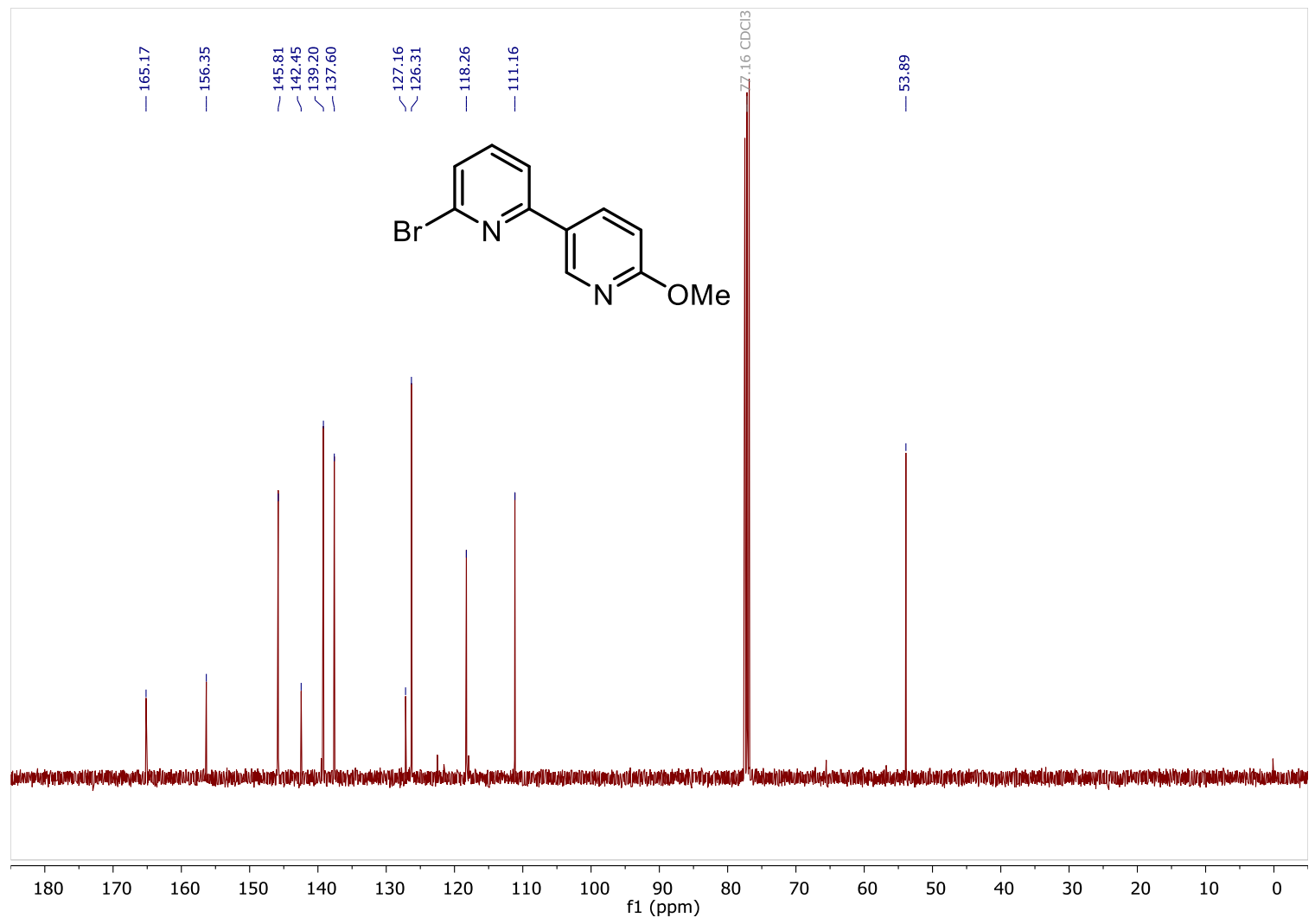

S93 
${ }^{1} \mathrm{H}$ NMR (500 MHz, $\mathrm{CDCl}_{3}$ ): 40

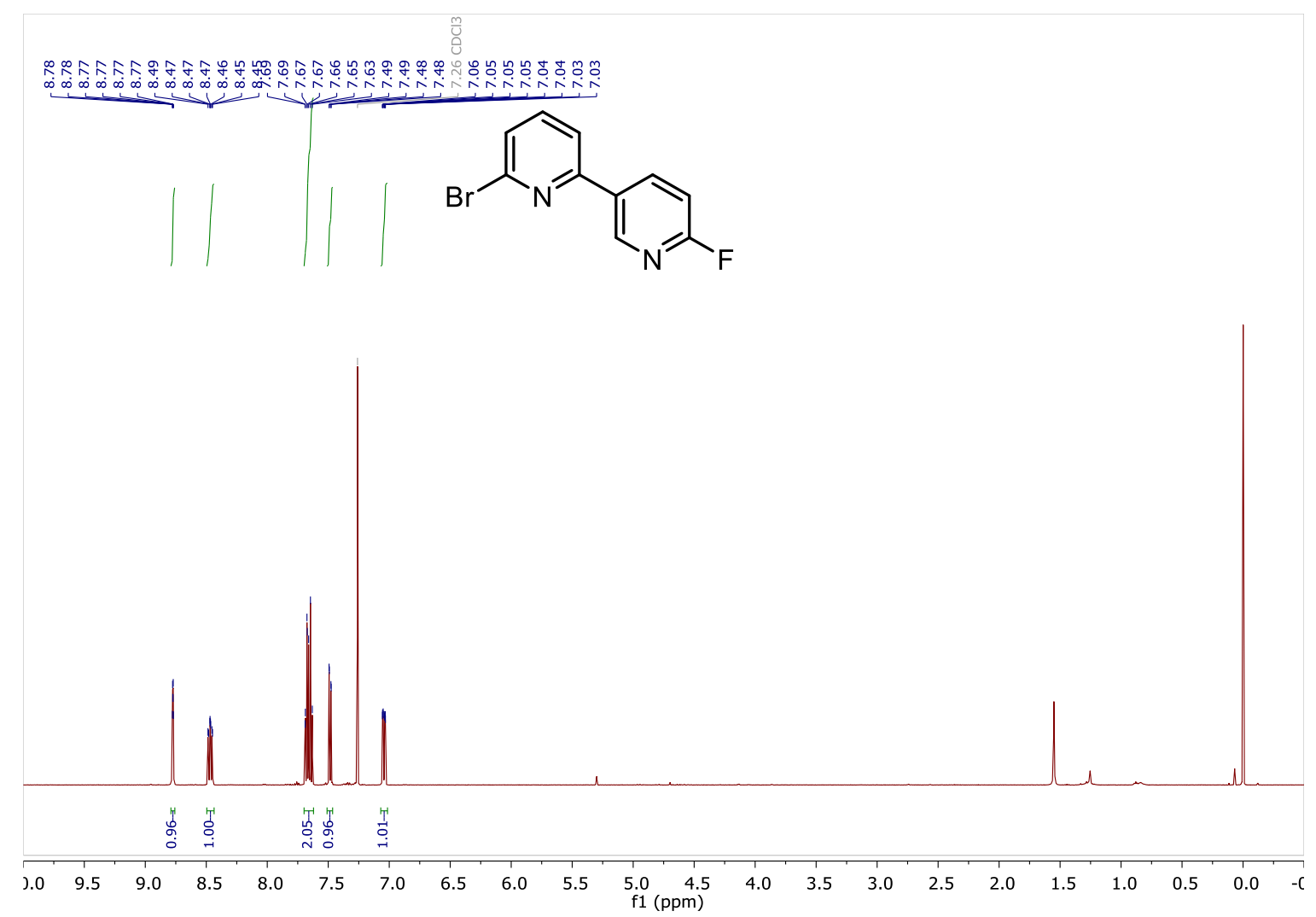

${ }^{13} \mathrm{C}$ NMR (101 MHz, $\left.\mathrm{CDCl}_{3}\right): 40$

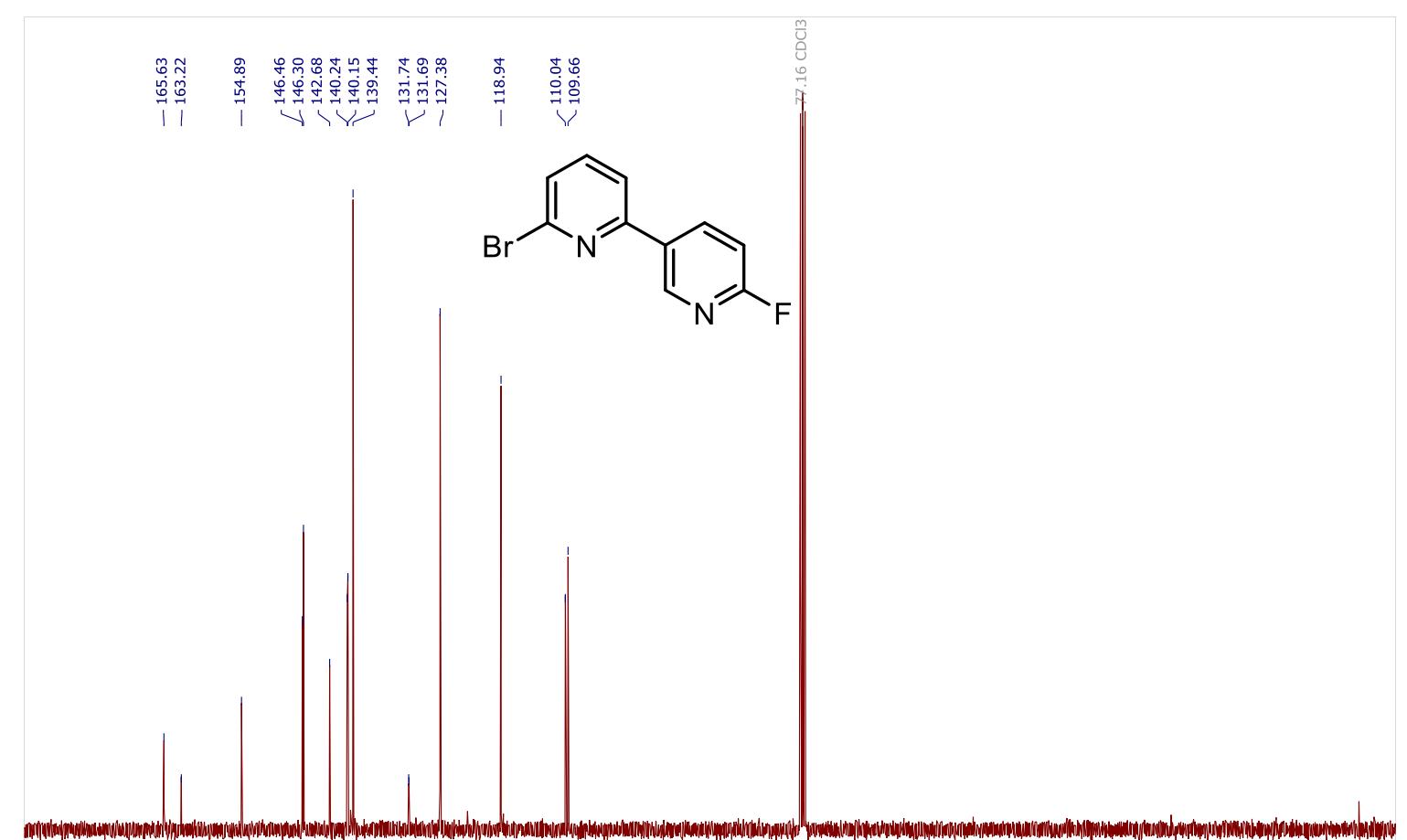

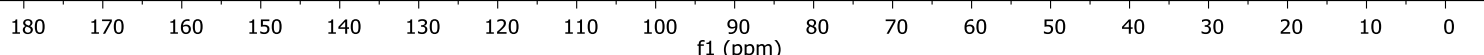


${ }^{1} \mathrm{H}$ NMR (500 MHz, $\mathrm{CDCl}_{3}$ ): 43

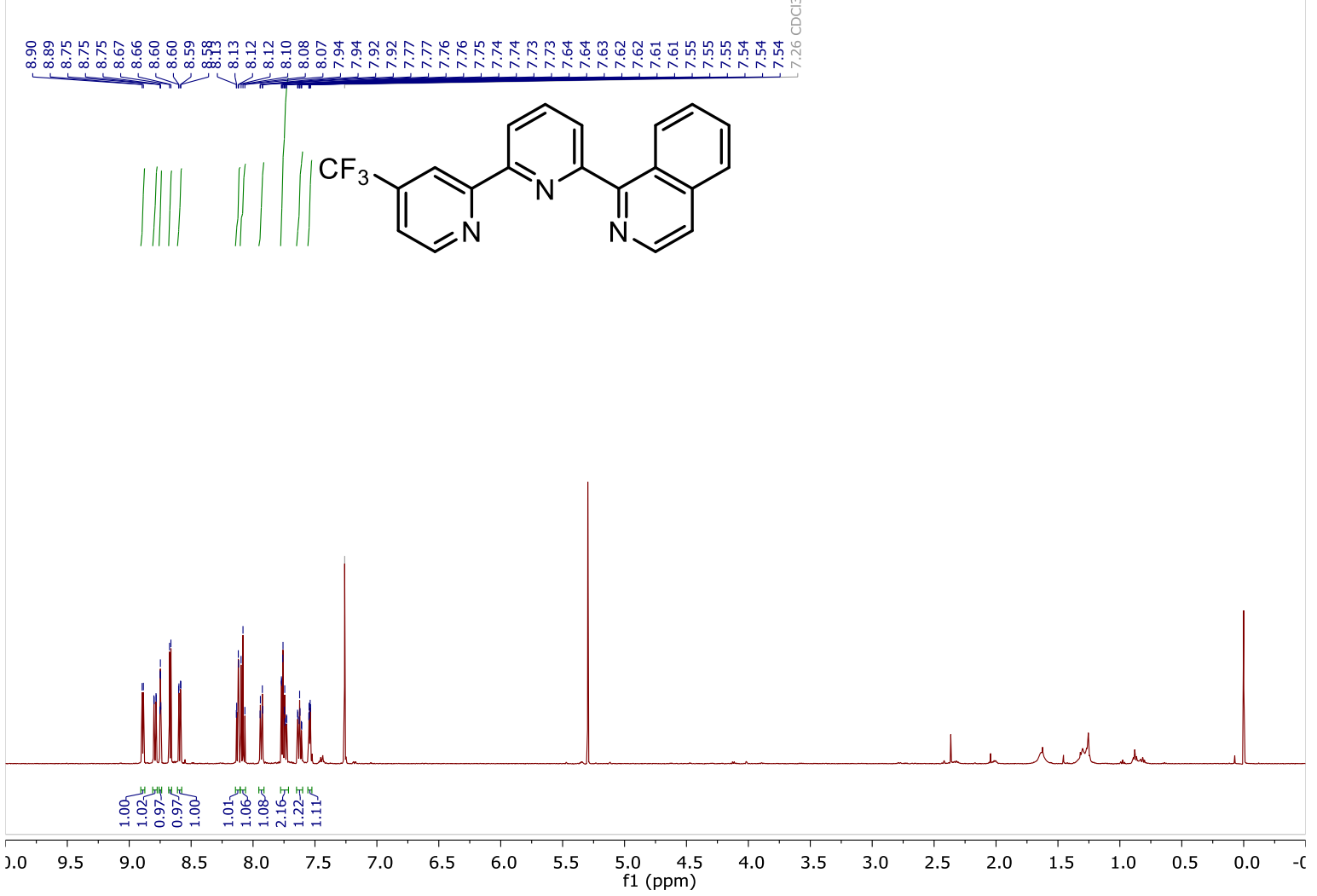

${ }^{13} \mathrm{C}$ NMR (126 MHz, $\left.\mathrm{CDCl}_{3}\right): 43$

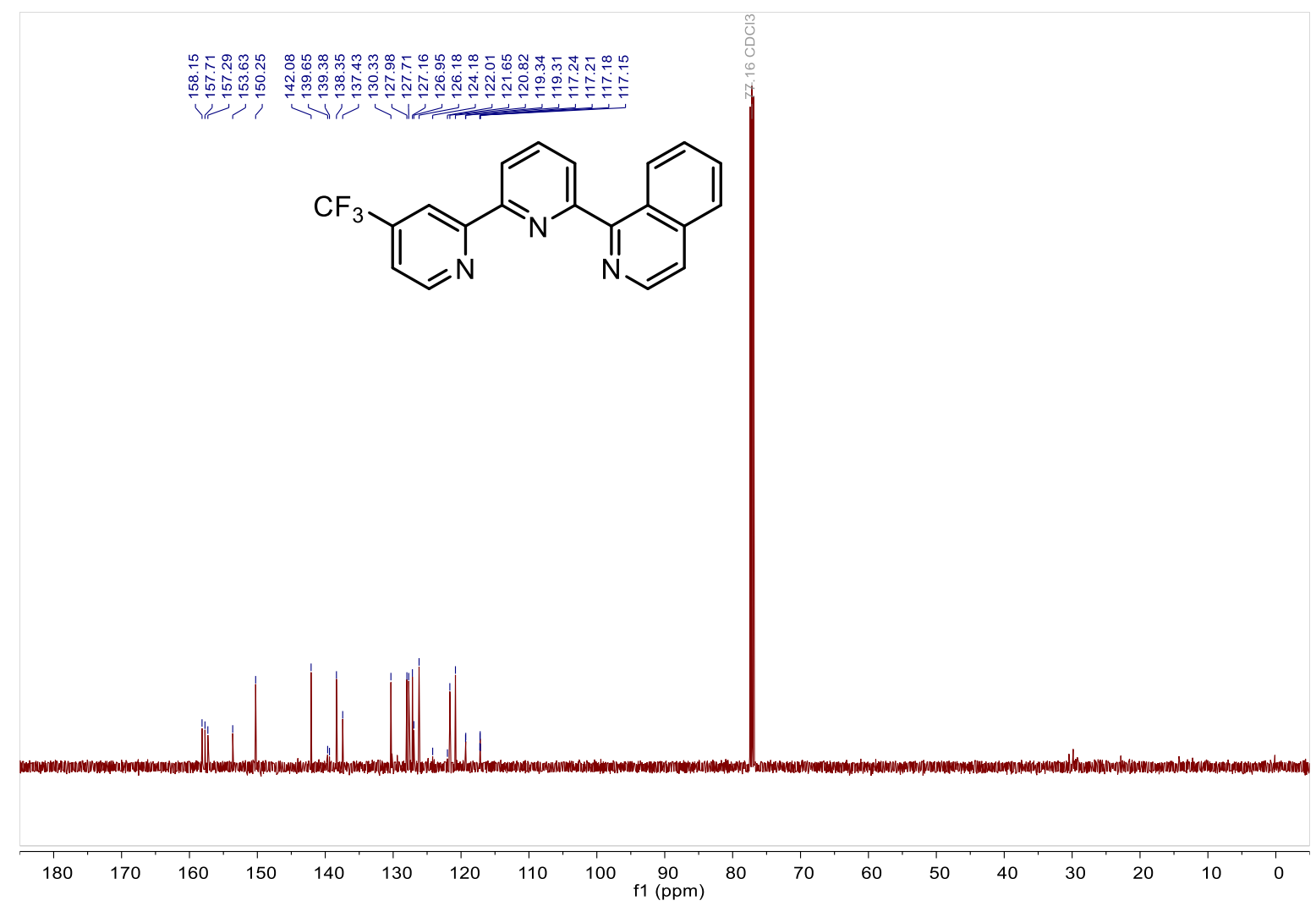




\section{References}

(1) Petersen, T. B.; Khan, R.; Olofsson, B. Metal-Free Synthesis of Aryl Esters from Carboxylic Acids and Diaryliodonium Salts. Org. Lett. 2011, 13 (13), 3462-3465. https://doi.org/10.1021/ol2012082.

(2) Hardy, M.; Struch, N.; Topić, F.; Schnakenburg, G.; Rissanen, K.; Lützen, A. Stepwise Construction of Heterobimetallic Cages by an Extended Molecular Library Approach. Inorg. Chem. 2018, 57 (7), 3507-3515. https://doi.org/10.1021/acs.inorgchem.7b02516.

(3) Grave, C.; Lentz, D.; Schäfer, A.; Samorì, P.; Rabe, J. P.; Franke, P.; Schlüter, A. D. ShapePersistant Macrocycles with Terpyridine Units: Synthesis, Characterization, and Structure in the Crystal. J. Am. Chem. Soc. 2003, 125 (23), 6907-6918. https://doi.org/10.1021/ja034029p.

(4) Khakyzadeh, V.; Rostami, A.; Veisi, H.; Shirmardi Shaghasemi, B.; Reimhult, E.; Luque, R.; Xia, Y.; Darvishi, S. Direct C-S Bond Formation: Via C-O Bond Activation of Phenols in a Crossover Pd/Cu Dual-Metal Catalysis System. Org. Biomol. Chem. 2019, 17 (18), 4491-4497. https://doi.org/10.1039/c9ob00313d.

(5) Liu, D.; Ma, H. X.; Fang, P.; Mei, T. S. Nickel-Catalyzed Thiolation of Aryl Halides and Heteroaryl Halides through Electrochemistry. Angew. Chem. Int. Ed. 2019, 58 (15), 5033-5037. https://doi.org/10.1002/anie.201900956.

(6) Moser, D.; Duan, Y.; Wang, F.; Ma, Y.; O’Neill, M. J.; Cornella, J. Selective Functionalization of Aminoheterocycles by a Pyrylium Salt. Angew. Chem. Int. Ed. 2018, 57 (34), 11035-11039. https://doi.org/10.1002/anie.201806271.

(7) Gao, G.; Xia, W.; Jain, P.; Yu, J.-Q. Pd(II)-Catalyzed C3-Selective Arylation of Pyridine with (Hetero)Arenes. Org. Lett. 2016, 18, 744-747. https://doi.org/10.1021/acs.orglett.5b03712.

(8) Markovic, T.; Rocke, B. N.; Blakemore, D. C.; Mascitti, V.; Willis, M. C. Pyridine Sulfinates as General Nucleophilic Coupling Partners in Palladium-Catalyzed Cross-Coupling Reactions with Aryl Halides. Chem. Sci. 2017, 8 (6), 4437-4442. https://doi.org/10.1039/c7sc00675f.

(9) Knapp, D. M.; Gillis, E. P.; Burke, M. D. A General Solution for Unstable Boronic Acids: SlowRelease Cross-Coupling from Air-Stable MIDA Boronates. J. Am. Chem. Soc. 2009, 131 (20), 6961-6963. https://doi.org/10.1021/ja901416p.

(10) Zucca, A.; Maidich, L.; Canu, L.; Petretto, G. L.; Stoccoro, S.; Cinellu, M. A.; Clarkson, G. J.; Rourke, J. P. Rollover-Assisted C(Sp2)-C(Sp3) Bond Formation. Chem. Eur. J. 2014, 20 (18), 5501-5510. https://doi.org/10.1002/chem.201304257.

(11) Cuperly, D.; Gros, P.; Fort, Y. First Direct C-2-Lithiation of 4-DMAP. Convenient Access to 
Reactive Functional Derivatives and Ligands. J. Org. Chem. 2002, 67 (1), 238-241. https://doi.org/10.1021/jo016064p.

(12) Zoppellaro, G.; Ivanova, A.; Enkelmann, V.; Geies, A.; Baumgarten, M. Synthesis, Magnetic Properties and Theoretical Calculations of Novel Nitronyl Nitroxide and Imino Nitroxide Diradicals Grafted on Terpyridine Moiety. Polyhedron 2003, 22 (14-17), 2099-2110. https://doi.org/10.1016/S0277-5387(03)00258-4.

(13) Trecourt, F.; Gervais, B.; Mongin, O.; Gal, C. Le; Mongin, F.; Queguiner, G. First Syntheses of Caerulomycin E and Collismycins A and C . A New Synthesis of Caerulomycin A. J. Org. Chem. 1998, 63, 2892-2897. https://doi.org/10.1021/jo972022i.

(14) Salamanca, V.; Toledo, A.; Albeniz, A. C. [2,2'-Bipyridin]-6(1H)-one, a Truly Cooperating Ligand in the Palladium-Mediated $\mathrm{C}-\mathrm{H}$ Activation Step: Experimental Evidence in the Direct C-3 Arylation of Pyridine. J. Am. Chem. Soc. 2018, 140, 17851-17856. https://doi.org/10.1021/jacs.8b10680.

(15) Holmstrøm, T.; Pedersen, C. M. Conformationally Switchable Glycosyl Donors. J. Org. Chem. 2019, 84 (21), 13242-13251. https://doi.org/10.1021/acs.joc.9b00830.

(16) Hoang, T. N. Y.; Humbert-Droz, M.; Dutronc, T.; Guénée, L.; Besnard, C.; Piguet, C. A Polyaromatic Terdentate Binding Unit with Fused 5,6-Membered Chelates for Complexing s-, p-, d-, and f-Block Cations. Inorg. Chem. 2013, 52 (9), 5570-5580. https://doi.org/10.1021/ic400526j.

(17) Ackermann, L.; Potukuchi, H. K.; Kapdi, A. R.; Schulzke, C. Kumada-Corriu Cross-Couplings with 2-Pyridyl Grignard Reagents. Chem. Eur. J. 2010, 16, 3300-3303. https://doi.org/10.1002/chem.201000032.

(18) Nagase, M.; Kuninobu, Y.; Kanai, M. 4-Position-Selective C-H Perfluoroalkylation and Perfluoroarylation of Six-Membered Heteroaromatic Compounds. J. Am. Chem. Soc. 2016, 138 (19), 6103-6106. https://doi.org/10.1021/jacs.6b01753.

(19) Zhang, E.; Tang, J.; Li, S.; Wu, P.; Moses, J. E.; Sharpless, K. B. Chemoselective Synthesis of Polysubstituted Pyridines from Heteroaryl Fluorosulfates. Chem. Eur. J. 2016, 22 (16), 56925697. https://doi.org/10.1002/chem.201600167.

(20) Lützen, A.; Hapke, M.; Staats, H.; Bunzen, J. Synthesis of Differently Disubstituted 2,2'Bipyridines by a Modified Negishi Cross-Coupling Reaction [ $\ddagger$ ]. Eur. J. Org. Chem. 2003, 3948-3957. https://doi.org/10.1002/ejoc.200300192.

(21) Liao, L. Y.; Kong, X. R.; Duan, X. F. Reductive Couplings of 2-Halopyridines without External 
Ligand: Phosphine-Free Nickel-Catalyzed Synthesis of Symmetrical and Unsymmetrical 2,2'Bipyridines. J. Org. Chem. 2014, 79 (2), 777-782. https://doi.org/10.1021/jo402084m.

(22) Laha, J. K.; Jethava, K. P.; Patel, S.; Patel, K. V. Intramolecular Acylation of Unactivated Pyridines or Arenes via Multiple C-H Functionalizations: Synthesis of All Four Azafluorenones and Fluorenones. J. Org. Chem. 2017, 82 (1), 76-85. https://doi.org/10.1021/acs.joc.6b02065.

(23) Qin, T.; Zhou, M.; Tsien, J. S(IV)-Mediated Unsymmetrical Heterocycle Cross-Couplings. Angew. Chem. Int. Ed. 2020, 1-6. https://doi.org/10.1002/anie.201915425.

(24) Liu, Y.; Bergès, J.; Zaid, Y.; Chahdi, F. O.; Van Der Lee, A.; Harakat, D.; Clot, E.; Jaroschik, F.; Taillefer, M. Aerobic and Ligand-Free Manganese-Catalyzed Homocoupling of Arenes or Aryl Halides via in Situ Formation of Aryllithiums. J. Org. Chem. 2019, 84 (7), 4413-4420. https://doi.org/10.1021/acs.joc.8b02834.

(25) Ogawa, A.; Oohora, K.; Gu, W.; Hayashi, T. Electrochemical CO2 Reduction by a Cobalt Bipyricorrole Complex: Decrease of an Overpotential Value Derived from Monoanionic Ligand Character of the Porphyrinoid Species. Chem. Commun. 2019, 55, 493-496. https://doi.org/10.1039/c8cc08876d. 